\title{
Non-Abelian Composition Factors of $m$-RATIONAL GROUPS
}

\author{
by
}

Stephen J. Trefethen

A Dissertation Submitted to the Faculty of the Department of MATHEMATiCs

In Partial Fulfillment of the Requirements
For the Degree of
Doctor of Philosophy
In the Graduate College
The University of Arizona 


\section{THE UNIVERSITY OF ARIZONA GRADUATE COLLEGE}

As members of the Dissertation Committee, we certify that we have read the dissertation prepared by Stephen J. Trefethen entitled

Non-Abelian Composition Factors of $m$-Rational Groups

and recommend that it be accepted as fulfilling the dissertation requirement for the Degree of Doctor of Philosophy.

DATE: MAY 4, 2016

Pham HuU TieP

DATE: MAY 4, 2016

KLAUS LUX

DATE: MAY 4, 2016

BRYDEN CAIS

DATE: MAY 4, 2016

ROMYAR SHARIFI

FINAL APPROVAL AND ACCEPTANCE OF THIS DISSERTATION IS CONTINGENT UPON THE CANDIDATE'S SUBMISSION OF THE FINAL COPIES OF THE DISSERTATION TO the Graduate College.

I HEREBY CERTIFY THAT I HAVE READ THIS DISSERTATION PREPARED UNDER MY DIRECTION AND RECOMMEND THAT IT BE ACCEPTED AS FULFILLING THE DISSERTATION REQUIREMENT.

DATE: MAY 4, 2016

Pham HuU TieP 


\section{Statement By Author}

THIS DISSERTATION HAS BEEN SUBMITTED IN PARTIAL FULFILLMENT OF REQUIREMENTS FOR AN ADVANCED DEgREE AT THE UNIVERSITY OF ARIZONA AND IS DEPOSITED IN THE UNIVERSITY LiBRARY TO BE MADE AVAILABLE TO BORROWERS UNDER RULES OF THE LIBRARY.

BRIEF QUOTATIONS FROM THIS DISSERTATION ARE ALLOWABLE WITHOUT SPECIAL PERMISSION, PROVIDED THAT ACCURATE ACKNOWLEDGMENT OF SOURCE IS MADE. REQUESTS FOR PERMISSION FOR EXTENDED QUOTATION FROM OR REPRODUCTION OF THIS MANUSCRIPT IN WHOLE OR IN PART MAY BE GRANTED BY THE HeAd of the Major Department or the Dean of the Graduate College WHEN IN HIS OR HER JUDGMENT THE PROPOSED USE OF THE MATERIAL IS IN THE INTERESTS OF SCHOLARSHIP. IN ALL OTHER INSTANCES, HOWEVER, PERMISSION MUST BE OBTAINED FROM THE AUTHOR.

Signed: Stephen J. Trefethen 


\section{ACKNOWLEDGMENTS}

First and foremost, I would like to thank my advisor, Professor Pham Huu Tiep, for all of his guidance and encouragement. He has taught me nearly everything that I need to know to complete my dissertation, has presented me with countless opportunities for professional development, and has always offered his full support. His unwavering patience and incessant kindness will never be forgotten, and I will forever be grateful to him. I would also like to thank Professor Klaus Lux for sparking my interest in group and representation theory, as well as for introducing me to computer programming with GAP. His numerous helpful sessions have been essential in completing my thesis. I want to thank my comprehensive exam and dissertation committee members, Professor Bryden Cais and Professor Romyar Sharifi, for your help in the dissertation process, and for the knowledge you gave me in my first years at the University of Arizona, steering me toward algebra. Thank you as well to Professor Eamonn O'Brien (Auckland) for your computational expertise, and helpful contributions to my thesis. I would like to extend my gratitude to Professor Gabriel Navarro for writing letters of recommendation for me in my search for employment, as well as providing insightful feedback on my dissertation.

My doctoral study was funded in part by NSF grant DMS-1201374, a research assistantship obtained by Professor Tiep. I am grateful for the opportunities that resulted from this grant. I am also thankful for my teaching assistantships at the University of Arizona. Working with my students solidified my love for mathematics, and I have many fond memories from the classroom.

Thank you to my family for their tremendous support. I can feel your love all the way from New York and Maine, and your kind words have always given me the encouragement to push through the most difficult times. You taught me the value of hard work, responsibility, and perseverance. I love you all very much! 
Table of Contents

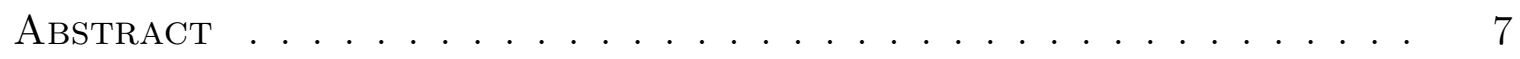

1. INTRODUCTION $\ldots \ldots \ldots \ldots \ldots$

1.1. $m$-Rational Groups . . . . . . . . . . . . . . . . . . . 11

1.2. $\quad \ell$-Brauer $m$-Rational Groups . . . . . . . . . . . . . . . 13

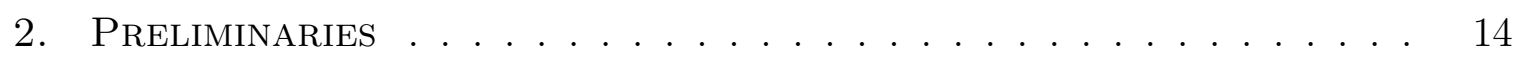

2.1. Groups of Lie Type. . . . . . . . . . . . . . . . . . . . 14

2.1.1. The Classical Groups of Lie Type. . . . . . . . . . 16

2.1.2. The Exceptional Groups of Lie Type . . . . . . . . . 22

2.2. Representations of Groups of Lie Type . . . . . . . . . . . . . 24

2.2.1. Some Character Theoretic Results . . . . . . . . 25

2.2.2. Fields of Values of Characters . . . . . . . . . . . . . 29

2.2.3. Some Deligne-Lusztig Theory . . . . . . . . . . . . 34

2.3. Modular Character Theory $\ldots \ldots \ldots \ldots$

2.3.1. Blocks . . . . . . . . . . . . . . . 38

2.4. Structure of Maximal Tori . . . . . . . . . . . . . . . . . . 41

2.4.1. The Classical Groups of Lie Type. . . . . . . . . . . 41

2.4.2. The Exceptional Groups of Lie Type . . . . . . . . 45

3. ZSIGMONDY PRIMES . . . . . . . . . . . . . . . . . . 53

3.1. Definitions and Known Facts . . . . . . . . . . . . 53

3.2. The Case $N=2 \ldots \ldots \ldots \ldots \ldots \ldots$

3.3. Application to the Special Linear Group . . . . . . . . . . . . 62

$4 . \quad m$-RATIONAL GROUPS . . . . . . . . . . . . . . . . . . 66

4.1. Reduction To Simple Groups . . . . . . . . . . . . . . 67

4.2. The Linear Groups . . . . . . . . . . . . . . . . . . . 72

4.3. The Unitary Groups . . . . . . . . . . . . . . . . . . . . . 78

4.4. The Symplectic and Odd-Dimensional Orthogonal Groups . . . 81

4.5. The Even-Dimensional Orthogonal Groups . . . . . . . . . . . . 84

4.6. The Exceptional Groups . . . . . . . . . . . . . . . . . . . 87

4.7. The Main Results. . . . . . . . . . . . . . . . . . . . . . . . . 91

$5 . \quad \ell$-BRAUER $m$-RATIONAL GROUPS $\ldots \ldots \ldots \ldots$

5.1. Reduction To Simple Groups . . . . . . . . . . . . . . . . . 94

5.2. The Linear Groups . . . . . . . . . . . . . . . . . . . . . . . . . . . 99

5.3. The Unitary Groups . . . . . . . . . . . . . . . . . . . 114 
TABle of Contents-Continued

5.4. The Symplectic and Odd-Dimensional Orthogonal Groups . . . 121

5.5. The Even-Dimensional Orthogonal Groups . . . . . . . . . . . . 130

5.6. The Exceptional Groups . . . . . . . . . . . . . . . . . . . 137

5.7. The Main Results . . . . . . . . . . . . . . . . . . . . . . . . . . 142

A. APPENDIX . . . . . . . . . . . . . . . . . . . . 152

A.1. CHEVIE Character Table . . . . . . . . . . . . . . . . . . . 152

A.2. Computations . . . . . . . . . . . . . . . . . . . 158

A.2.1. $\mathrm{PSL}_{n}(q)$. . . . . . . . . . . . . . . . . . . . . . . . . . . . . . . . . . . . . .

A.2.2. $\mathrm{PSU}_{n}(q)$. . . . . . . . . . . . . . . . . . . 160

A.2.3. $\mathrm{PSp}_{2 n}(q)$ and $\mathrm{O}_{2 n+1}(q)$. . . . . . . . . . . . . 164

A.2.4. $\mathrm{O}_{2 n}^{+}(q) \ldots \ldots \ldots \ldots \ldots$

A.2.5. $\mathrm{O}_{2 n}^{-}(q) \ldots \ldots \ldots \ldots \ldots \ldots$

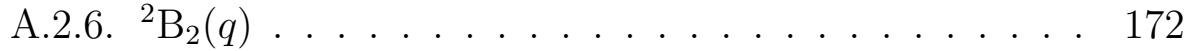

A.2.7. ${ }^{3} \mathrm{D}_{4}(q)$. . . . . . . . . . . . . . . . . . . . . . 173

A.2.8. $\mathrm{E}_{6}(q)$. . . . . . . . . . . . . . . . . . . . 173

A.2.9. ${ }^{2} \mathrm{E}_{6}(q)$. . . . . . . . . . . . . . . . . . . . . 174

A.2.10. $\mathrm{E}_{7}(q)$

A.2.11. $\mathrm{E}_{8}(q)$

A.2.12. $\mathrm{F}_{4}(q)$. . . . . . . . . . . . . . . . . 175

A.2.13. ${ }^{2} \mathrm{~F}_{4}(q)$. . . . . . . . . . . . . . . . . . . 176

A.2.14. $\mathrm{G}_{2}(q) \ldots \ldots \ldots \ldots \ldots \ldots$

A.2.15. ${ }^{2} \mathrm{G}_{2}(q) \ldots \ldots \ldots \ldots$. . . . . . . . . . . . . . . 177

REFERENCES . . . . . . . . . . . . . . . . . . . . . . . 178 


\section{ABstract}

In this thesis, we discuss several problems in the representation theory of finite groups of Lie type. In Chapter 2, we will give essential background material that will be useful for the entirety of the thesis. We will investigate the construction of groups of Lie type, as well as their representations. We will define the field of values of a character afforded by a representation, and state useful results concerning these fields. In Chapter 3, we examine Zsigmondy primes and their existence, a necessary ingredient in proving our main results. In Chapters 4 and 5, we describe our main results in the ordinary and modular cases, which we now summarize.

A finite group $G$ is said to be $m$-rational, for a fixed positive integer $m$, if $[\mathbb{Q}(\chi)$ : Q] $\mid m$ for any irreducible character $\chi \in \operatorname{Irr}(G)$. In 1976, R. Gow studied the structure of solvable rational groups (i.e. $m=1$ ), and found that the possible composition factors of a solvable rational group are cyclic groups of prime order $p \in\{2,3,5\}$, [22]. Just over a decade later, W. Feit and G. Seitz classified the possible non-abelian composition factors of (non-solvable) rational groups, [14]. In 2008, J. Thompson found an upper bound of $p \leq 13$ for the order of the possible cyclic composition factors of an arbitrary rational group, and conjectured that the bound can be improved to $p \leq 5$, 46]. More recently, J. McKay posed the question of determining the structure of quadratic rational groups (i.e. $m=2$ ). J. Tent studied the cyclic composition factors of solvable quadratic rational groups in 2013, [45]. In Chapter 4, we answer McKay's question concerning non-abelian composition factors, and generalize our results to non-solvable $m$-rational groups.

Modular character theory was founded by R. Brauer in the 1930's, and has been useful in proving historical results including the classification of finite simple groups. In Chapter 5, we prove the modular version of our results. Though our conclusions are similar to those found in the complex case, the methods for proving the results 
are typically much more complicated. 


\section{INTRODUCTION}

The study of group theory was motivated by geometry and the desire to understand symmetry, as well as by number theory and the theory of algebraic equations. Symmetry occurs in art, communication networking, crystals and atoms, and the body structure of most organisms. Consequently, group theory is a highly active field of research, with applications in pure and applied mathematics, chemistry, physics, cryptography, and coding theory. Representation theory gives a link between arbitrary groups in nature and a collection of well understood objects, namely matrices. These representations decompose into irreducible representations. We can then take the trace of the image of group elements under our irreducible representations, and this affords an irreducible character of our group. We are interested in extracting as much information from the collection of irreducible characters as possible, in an effort to identify the structure of arbitrary groups.

From the Jordan-Hölder theorem, it is known that every finite group $G$ has a composition series, $1=N_{0} \triangleleft N_{1} \triangleleft \cdots \triangleleft N_{k}=G$ with each $N_{i+1} / N_{i}$ simple, and further, the set of composition factors $\left\{N_{i+1} / N_{i}\right\}$ is unique up to isomorphism and reordering. This suggests that many problems concerning arbitrary groups can be reduced to those of simple groups. In 2004, the monumental Classification of Finite Simple Groups was completed, and has since been used to prove many theorems in finite group theory. The Classification states that every finite simple group is one of the following:

- A cyclic group of prime order

- An alternating group $A_{n}$ with $n \geq 5$

- One of 26 sporadic groups 
- A finite group of Lie type

According to this classification, "most" finite simple groups are groups of Lie type. Within the collection of groups of Lie type, we find the classical groups and exceptional groups. The classical groups can be realized nicely as groups of matrices over finite fields, and thus, we can take a hands on approach when proving character theoretic results. However, the exceptional groups do not have such nice realizations, and we must take a more theoretical approach. We will discuss the construction of groups of Lie type in Chapter 2.

As mentioned above, our dissertation results rely on the representations of finite groups. To be more precise, a complex representation of a finite group $G$ is a homomorphism $\Phi: G \rightarrow \mathrm{GL}_{n}(\mathbb{C})$, where $\mathrm{GL}_{n}(\mathbb{C})$ is the group of invertible $n$ by $n$ matrices with entries in $\mathbb{C}$, the complex numbers. Such a representation uniquely defines a $\mathbb{C} G$-module $V$, with the action of $G$ on $V$ given by the representation. The representation $\Phi$ is then said to be irreducible if $V$ is irreducible as a $\mathbb{C} G$-module, i.e. $\quad V$ has exactly two $\mathbb{C} G$-submodules. Furthermore, every $\mathbb{C} G$-module $W$ has a composition series $0=W_{1} \subseteq W_{2} \subseteq \cdots \subseteq W_{m}=W$ with $W_{i+1} / W_{i}$ irreducible. In this sense, we may decompose every $\mathbb{C} G$-representation into a direct sum of irreducible representations.

Now, we may consider the class function $\chi: G \rightarrow \mathbb{C}$ defined by $\chi(g)=\operatorname{tr}(\Phi(g))$. We call this the character afforded by the representation $\Phi$, and denote the collection of all irreducible characters of $G$ by $\operatorname{Irr}(G)$. It is well known (see for example [25]) that $\chi$ takes values in $\mathbb{Q}(\zeta)$, where $\zeta$ is a primitive $|G|$-th root of unity, and $|G|$ is the order of the group $G$. However, if we examine the character tables of various groups, it is clear that the fields of values of the irreducible characters are generally much smaller. For example, every irreducible character of a symmetric group, $S_{n}$, takes values solely in the rational numbers, despite having group order $\left|S_{n}\right|=n$ !. We define a finite group $G$ to be $m$-rational for a fixed positive integer $m$ if $[\mathbb{Q}(\chi): \mathbb{Q}] \mid m$ for all 
irreducible characters $\chi \in \operatorname{Irr}(G)$. In Section 1.1, we briefly describe the history of the study of rational groups and give the motivation for the definition of $m$-rationality. In Chapter 4, we willl discuss our results on the structure of $m$-rational groups.

Modular representation theory was developed in the 1930's by Richard Brauer. Modular representation theory has applications in various areas of mathematics, including group theory, number theory, and algebraic geometry. It is critically used in the proof of the classification of finite simple groups, in Wiles' proof of Fermat's last theorem, and in Quillen's proof of the Adams conjecture. In this branch of representation theory, we now consider the representations $\Phi: G \rightarrow \mathrm{GL}(V)$, where $V$ is a finite-dimensional vector space over a field $\mathbb{F}$ of characteristic $\ell>0$. As before, we collect the $\ell$-Brauer characters afforded by the irreducible modular representations into a finite set, $\operatorname{IBr}_{\ell}(G)$. We define a finite group $G$ to be $\ell$-Brauer $m$-rational for a fixed prime $\ell$ and a fixed positive integer $m$ if $[\mathbb{Q}(\phi): \mathbb{Q}] \mid m$ for all $\phi \in \operatorname{IBr}_{\ell}(G)$. In Section 1.2 , we briefly explain that $m$-rationality and $\ell$-Brauer $m$-rationality are independent, in that neither implies the other, and in Chapter 5, we give our results on $\ell$-Brauer $m$-rational groups.

\section{1. $m$-Rational Groups}

In this section, we give a brief overview of the history and motivation for studying $m$-rational groups.

There is a strong connection between the conjugacy classes of a rational group and the fields of values of the characters of a rational group. In particular, a group is rational if for any $x \in G$ and each integer $k$ with $(k,|G|)=1, x$ is conjugate to $x^{k}$. Using elementary Galois theory, we can see that this is equivalent to every irreducible character of $G$ taking values only in $\mathbb{Q}$. The study of rational groups dates back to the 1970's when R. Gow determined the possible cyclic composition factors of any solvable rational group, [22, Corollary 2]: 
Theorem 1.1.1. The order of any solvable rational group has the form $2^{a} 3^{b} 5^{c}$.

Just over a decade later, W. Feit and G. Seitz proved the following result for (non-solvable) rational groups [14, Theorem B]:

Theorem 1.1.2. Let $S$ be a noncyclic finite simple group. Then $S$ is a composition factor of a rational group if and only if $S$ is isomorphic to an alternating group of one of the following groups:

1. $\mathrm{PSp}_{4}(3), \mathrm{Sp}_{6}(2), O_{8}^{+}(2)$,

2. $\mathrm{PSL}_{3}(4), \mathrm{PSU}_{4}(3)$.

Using this result of Feit and Seitz, J. Thompson nearly classified the possible composition factors of non-solvable rational groups, [46, Theorem 1.1]:

Theorem 1.1.3. If $p$ is a prime such that some rational finite group has a composition factor of order $p$, then $p \leq 11$.

He further conjectured that the theorem can be improved to $p \leq 5$. There are no known rational groups with a composition factor of order 7 or 11 , but there is no proof to eliminate these as possibilities either.

Recently, J. McKay posed the following question concerning 2-rational (i.e. quadratic rational) groups:

Problem 1. Is it possible to determine the potential composition factors of quadratic rational groups?

In 2013, J. Tent solved this problem in that case that $G$ is a solvable group, [45]:

Theorem 1.1.4. If $G$ is a quadratic rational solvable group, then the prime divisors of $|G|$ lie in $\{2,3,5,7,13\}$.

In Chapter 4, we answer McKay's problem concerning non-abelian composition factors, and extend our results to (non-solvable) $m$-rational groups. 


\section{2. $\ell$-Brauer $m$-Rational Groups}

A natural question that arises when discussing the fields of values of ordinary characters is "Does the same hold true for modular characters?" In the case of $m$-rationality, we find that the problems are essentially independent. There are certainly $m$-rational groups that are not $\ell$-Brauer $m$-rational (e.g. $\mathrm{SL}_{2}(7)$ with $\ell=7$ is quadratic rational, but is only 7 -Brauer 4 -rational), and there are $\ell$-Brauer $m$-rational groups that are not $m$-rational (e.g. $A_{5}$ with $\ell=5$ is 5 -Brauer rational but is quadratic rational). There are a few instances when $m$-rational groups are necessarily $\ell$-Brauer $m$-rational:

Definition 1.2.1. A finite group $G$ is said to be $\ell$-solvable if a composition series for $G$ has factors that are either $\ell$-groups or $\ell^{\prime}$-groups.

Theorem 1.2.2 (Fong-Swan-Isaacs). Every irreducible Brauer character of an $\ell$ solvable group $G$ lifts to an irreducible ordinary character of $G$ with values in $\mathbb{Q}\left(e^{\frac{2 i \pi}{r}}\right)$ for some $r$ coprime to $\ell$. Therefore, every $\ell$-solvable $m$-rational group is $\ell$-Brauer m-rational.

Further, we know that every irreducible $\ell$-Brauer character is an integral linear combination of the restrictions of the ordinary irreducible characters to the $\ell$-regular (i.e. $\left.\ell^{\prime}\right)$ conjugacy classes of $G$. Therefore, it is easy to see that rational groups are necessarily $\ell$-Brauer rational.

It appears that $\ell$-Brauer $m$-rationality has not been studied before, perhaps since modular representation theory of groups of Lie type tends to be more difficult than ordinary representation theory. Nonetheless, we are able to produce similar results to those found in the ordinary case, which we present in Chapter 5. Further, we will give a list of the possible composition factors of $\ell$-Brauer rational groups (i.e. $m=1$ ). 


\section{PRELIMINARIES}

In this chapter, we introduce the notation and background material that will be used in the subsequent chapters. As our main results depend on the representation theory of the finite groups of Lie type, we give a detailed description of their construction. We will discuss the ordinary and modular representations of these groups, giving many well-known, yet critical, results from character theory and Clifford theory. We will provide a brief introduction to semisimple elements of linear algebraic groups and Deligne-Lusztig theory, another essential component of our main thesis results. Finally, we will discuss the known structure of the finite maximal tori in the finite groups of Lie type.

\subsection{Groups of Lie Type}

Suppose that $p$ is a prime and $q$ is a power of $p$. Let $k:=\overline{\mathbb{F}}_{p}$, the algebraic closure of the finite field of $p$ elements. A closed (with respect to the Zariski topology) subgroup $\mathcal{G} \leq \mathrm{GL}_{n}(k)$ is called a connected reductive (linear) algebraic group if the unipotent radical (i.e. the maximal connected normal unipotent subgroup) of $\mathcal{G}$ is trivial and $\mathcal{G}$ is connected. We say that $\mathcal{G}$ is semisimple if its radical (i.e. the maximal connected normal solvable subgroup) is trivial, and $\mathcal{G}$ is simple if it has no proper nontrivial closed normal subgroups. A Steinberg endomorphism $F: \mathcal{G} \rightarrow \mathcal{G}$ is a morphism of algebraic groups such that $F^{k}$ is the standard Frobenius map $F_{q}: \mathcal{G} \rightarrow \mathcal{G}:\left(a_{i j}\right) \mapsto$ $\left(a_{i j}^{q}\right)$ for some $k$. More generally, a Frobenius map, $F: \mathcal{G} \rightarrow \mathcal{G}$, is a morphism of algebraic groups such that the subgroup of elements fixed by $F$ is a finite group.

Assume $\mathcal{G}$ is a connected reductive algebraic group over $\overline{\mathbb{F}}_{p}$, and let $F$ be a Frobenius map on $\mathcal{G}$. The subgroup

$$
\mathcal{G}^{F}:=\{g \in \mathcal{G} \mid F(g)=g\}
$$


is a finite group, called a finite reductive group, or a finite group of Lie type. We may also construct the (simple) finite groups of Lie type through Lie algebras, $\mathcal{L}$, over a field $K$. To do this, we follow the notation of [7], Chapter 4.

Let $\mathcal{L}$ be a simple Lie algebra and $K$ be a field. For each root $r$ of $\mathcal{L}$ and each element $t \in K$, set $x_{r}(t):=\exp \left(\operatorname{tad} e_{r}\right)$, where $e_{r}$ is a root vector corresponding to the root $r$. Let $\mathcal{L}(K)$ be the group of automorphisms of $\mathcal{L}$ generated by these elements $x_{r}(t)$. Then $\mathcal{L}(K)$ is a finite simple Chevalley group of Lie type. Further, $\mathcal{L}(K)$ is determined up to isomorphism by the simple Lie algebra $\mathcal{L}$ and the field $K$. Using the results of Killing and Cartan, it can be shown that there are only finitely many types of (necessarily indecomposable) root systems that generate a simple Lie algebra, namely:

Theorem 2.1.1. Let $\Phi$ be an indecomposable root system that generates a simple Lie algebra. Then $\Phi$ is isomorphic to one of the following: $A_{n}, B_{n}, C_{n}, D_{n}, E_{6}, E_{7}, E_{8}, F_{4}, G_{2}$ with the corresponding Dynkin diagram:

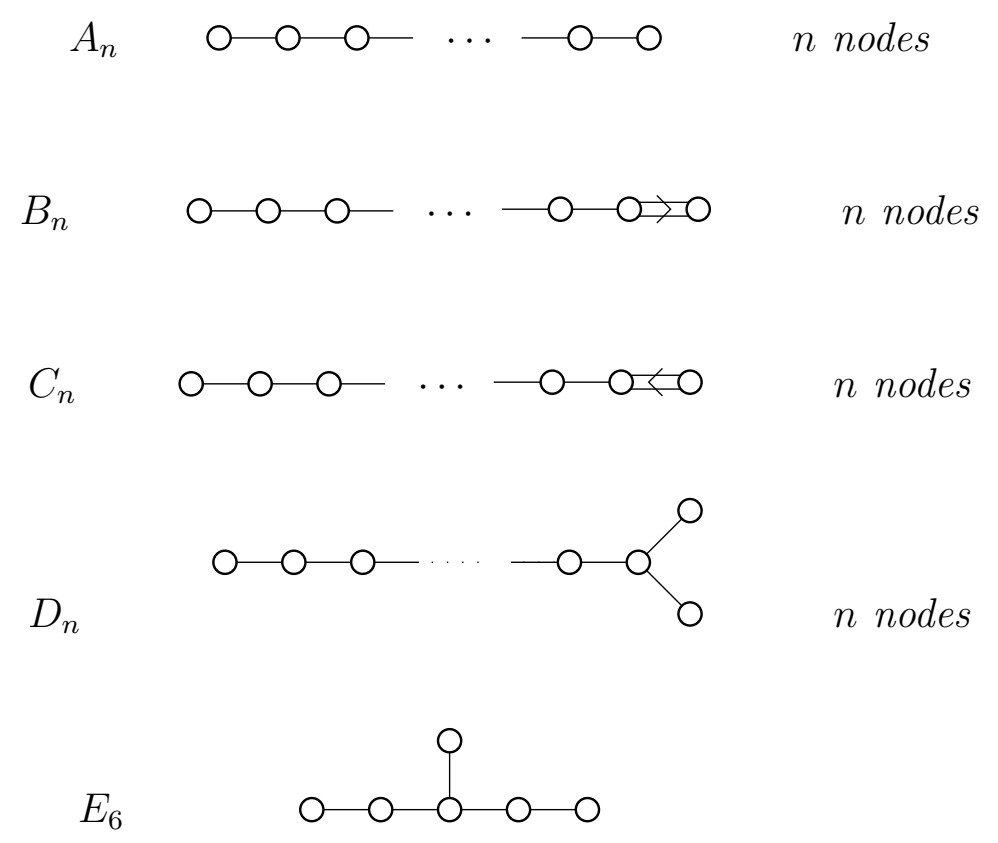




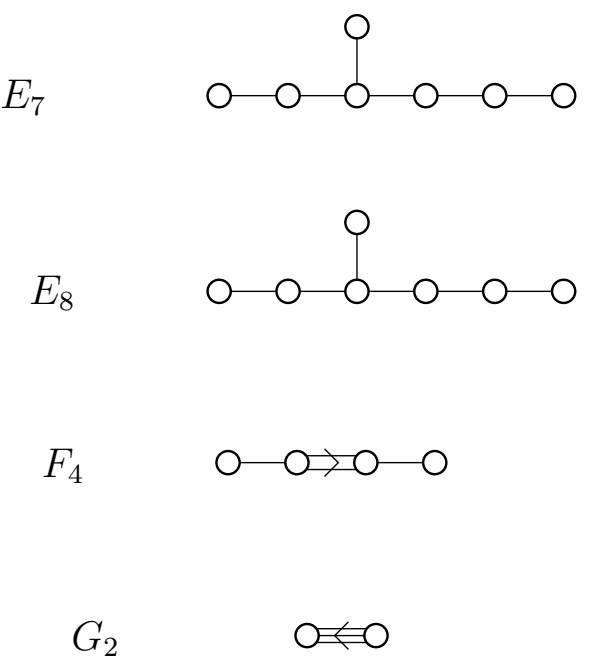

Hence, we obtain the finite simple Chevalley groups of Lie type. Further, using the automorphisms of the Dynkin diagrams, we may obtain twisted versions of the finite Chevalley groups.

\subsubsection{The Classical Groups of Lie Type}

1. Type $A_{n}, n \geq 1$ : In this case, we have an indecomposable root system given by the following Dynkin diagram:

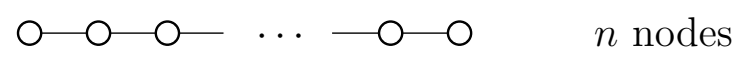

As described above, this yields the finite simple Chevalley group $A_{n}(q)$ by set$\operatorname{ting} K=\mathbb{F}_{q}$.

We may also construct this finite simple group as a group of matrices over a finite field: Let $V=\mathbb{F}_{q}^{n+1}$ be the $n+1$-dimensional vector space over the finite field $\mathbb{F}_{q}$. By choosing a basis, we may identify $\mathrm{GL}(V)$, the set of non-singular $\mathbb{F}_{q}$-linear transformations of $V$ with $\mathrm{GL}_{n+1}(q)$, the set of invertible $n+1 \times n+1$ matrices with entries in $\mathbb{F}_{q}$. We define $\mathrm{SL}_{n+1}(q)$ to be the (normal) subgroup of 
$\mathrm{GL}_{n+1}(q)$ consisting of matrices in $\mathrm{GL}_{n+1}(q)$ with determinant 1 . The set of nonzero diagonal matrices in $\mathrm{SL}_{n+1}(q)$ form the center of $\mathrm{SL}_{n+1}(q)$, and we define $\mathrm{PSL}_{n+1}(q):=\mathrm{SL}_{n+1}(q) / Z\left(\mathrm{SL}_{n+1}(q)\right)$. These groups are simple for all pairs $(n, q)$ except $(1,2)$ and $(1,3)$. By Theorem 11.3.2 of [7], $A_{n}(q) \cong \operatorname{PSL}_{n+1}(q)$.

Finally, we may construct this finite simple group from an algebraic group as follows:

Recall that $k:=\overline{\mathbb{F}_{q}}$, and set $F$ to be the standard Frobenius endomorphism. Let $\mathcal{G}=\mathrm{SL}_{n+1}(k)$. Then $\mathcal{G}^{F} \cong \mathrm{SL}_{n+1}(q)$, and $\mathrm{SL}_{n+1}(q) / Z\left(\mathrm{SL}_{n+1}(q)\right)=\operatorname{PSL}_{n+1}(q)$.

There is an obvious automorphism of order 2 of the Dynkin diagram when $n \geq 2$, and therefore, we obtain the twisted groups of type ${ }^{2} A_{n}$ as well. Setting $K=\mathbb{F}_{q^{2}}$, and following the construction from Lie algebras described above, we have the simple Chevalley group ${ }^{2} \mathrm{~A}_{n}\left(q^{2}\right)$.

We may construct these simple groups as groups of matrices as well: Let $f$ be the (unique up to similarity) unitary form (or Hermitian form) on $V=\mathbb{F}_{q^{2}}^{n+1}$. The general unitary group is the subgroup of $\mathrm{GL}(V)$ that are $f$-isometries. Choosing a suitable basis, we can identify this group with

$$
\mathrm{GU}_{n+1}(q)=\left\{g \in \mathrm{GL}_{n+1}\left(q^{2}\right) \mid\left(\bar{g}^{t}\right) g=I\right\},
$$

where $\bar{g}^{t}$ is the conjugate transpose of $g$. Taking the elements of determinant 1 and quotienting by the center, we obtain the projective special unitary group, $\operatorname{PSU}_{n+1}(q)$, which is simple except when $(n, q)=(2,2)$. By Theorem 11.3.2 of [7, ${ }^{2} \mathrm{~A}_{n}\left(q^{2}\right) \cong \operatorname{PSU}_{n+1}(q)$.

Finally, we may construct this finite simple group from an algebraic group as follows:

Set $F$ to be the inverse-transpose map composed with the standard Frobenius endomorphism. Let $\mathcal{G}=\mathrm{SU}_{n+1}(k)$. Then $\mathcal{G}^{F} \cong \mathrm{SU}_{n+1}(q)$, and

$$
\mathrm{SU}_{n+1}(q) / Z\left(\mathrm{SU}_{n+1}(q)\right)=\operatorname{PSU}_{n+1}(q) .
$$


2. Type $B_{n}, n \geq 2$ : It is known that when $q$ is even, $B_{n}(q)$ and $C_{n}(q)$ are isomorphic, and thus, we assume $q$ is odd. In this case, we have an indecomposable root system given by the following Dynkin diagram:

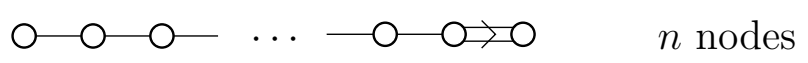

As described above, this yields the finite simple Chevalley group $B_{n}(q)$ by set$\operatorname{ting} K=\mathbb{F}_{q}$.

We may also construct these finite simple groups as a group of matrices: Let $Q$ be the (unique up to similarity) non-degenerate quadratic form on the vector space $V=\mathbb{F}_{q}^{2 n+1}$. Define $f_{Q}(v, w):=Q(v+w)-Q(v)-Q(w)$ to be the associated bilinear form. The general orthogonal group is the subgroup of $\mathrm{GL}(V)$ that are $Q$-isometries (i.e. $\{g \in \mathrm{GL}(V) \mid Q(g x)=Q(x), \forall x \in V\})$. Choosing a suitable basis for $V$, we see that this can be identified with

$$
\mathrm{GO}_{2 n+1}(q)=\left\{g \in \mathrm{GL}_{2 n+1}(q) \mid\left(g^{t}\right) J g=J\right\}
$$

where

$$
J=\left(\begin{array}{ccc}
0 & I_{n} & 0 \\
I_{n} & 0 & 0 \\
0 & 0 & 1
\end{array}\right)
$$

Taking elements of determinant 1, we obtain the special orthogonal group, $\mathrm{SO}_{2 n+1}(q)$. This group has a unique, simple (as we are assuming $q$ is odd) subgroup $\mathrm{O}_{2 n+1}(q)$ (sometimes written $\Omega_{2 n+1}(q)$ ) of index 2. By Theorem 11.3.2 of [7], $B_{n}(q) \cong \mathrm{O}_{2 n+1}(q)$.

Finally, we may construct these finite simple groups as the fixed points of an algebraic group:

Set $F$ to be the standard Frobenius endomorphism. Let $\mathcal{G}=\mathrm{SO}_{2 n+1}(k)$. Then $\mathcal{G}^{F} \cong \mathrm{SO}_{2 n+1}(q)$, and as above, this yields $\mathrm{O}_{2 n+1}(q)$. 
Notice that when $n=2$ (and we ignore the arrow), there is an automorphism of the Dynkin diagram, yielding a ${ }^{2} \mathrm{~B}_{2}$. These give rise to the Suzuki groups, which we will describe with the exceptional groups of Lie type.

3. Type $C_{n}, n \geq 2$ : In this case, we have an indecomposable root system given by the following Dynkin diagram:

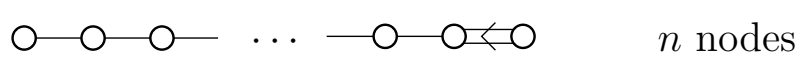

As described above, this yields the finite simple Chevalley group $C_{n}(q)$ by setting $K=\mathbb{F}_{q}$.

We may also construct these finite simple groups as a group of matrices: Let $f$ be the (unique up to similarity) symplectic form (i.e. non-degenerate skewsymmetric bilinear form) on $V=\mathbb{F}_{q}^{2 n}$. The symplectic group is the subgroup of $\mathrm{GL}(V)$ that are $f$-isometries. Choosing a suitable basis, we can identify this group with

$$
\operatorname{Sp}_{2 n}(q)=\left\{g \in \mathrm{GL}_{2 n}(q) \mid\left(g^{t}\right) P g=P\right\}
$$

where

$$
P=\left(\begin{array}{cc}
0 & I_{n} \\
-I_{n} & 0
\end{array}\right)
$$

Quotienting by the center, we obtain the projective symplectic group, $\operatorname{PSp}_{2 n}(q)$, which is simple except when $(n, q)=(2,2)$. The derived subgroup of $\operatorname{PSp}_{4}(2)$ is simple. By Theorem 11.3.2 of [7], $C_{n}(q) \cong \operatorname{PSp}_{2 n}(q)$.

Finally, we may construct these finite simple groups as the fixed points of an algebraic group:

Set $F$ to be the standard Frobenius endomorphism. Let $\mathcal{G}=\operatorname{Sp}_{2 n}(k)$. Then $\mathcal{G}^{F} \cong \operatorname{Sp}_{2 n}(q)$, and as above, this yields $\operatorname{PSp}_{2 n}(q)$. 
4. Type $D_{n}, n \geq 4$ : In this case, we have an indecomposable root system given by the following Dynkin diagram:

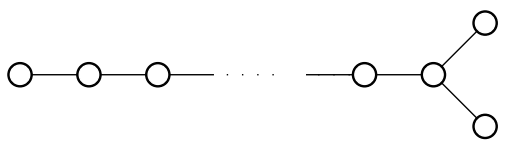

$n$ nodes

As described above, this yields the finite simple Chevalley group $D_{n}(q)$ by setting $K=\mathbb{F}_{q}$.

We may also construct these finite simple groups as a group of matrices: Let $Q$ be a non-degenerate quadratic form on a vector space $V$ of dimension $2 n$ over $\mathbb{F}_{q}$. Define

$$
f_{Q}(v, w)=(v, w)=Q(v+w)-Q(v)-Q(w)
$$

to be the associated bilinear form. As in [29, Proposition 2.5.3(i)], we assume that $V$ has a basis $\left\{e_{1}, \ldots, e_{n}, f_{1}, \ldots, f_{n}\right\}$ satisfying $Q\left(e_{i}\right)=Q\left(f_{i}\right)=0$ and $f_{Q}\left(e_{i}, f_{j}\right)=\delta_{i j}$ for all $i, j$. (In this case, we say that $V$ is of maximal Witt defect.) When $q$ is even, the bilinear form degenerates to a symplectic form, and the orthogonal group is the subgroup of the symplectic group, containing the $Q$-isometries. When $q$ is odd, the general orthogonal group is the subgroup of $\mathrm{GL}(V)$ that are $Q$-isometries (i.e. $\{g \in \mathrm{GL}(V) \mid Q(x g)=Q(x), \forall x \in V\}$ ). Choosing a suitable basis for $V$, we see that (for $q$ odd) this can be identified with

$$
\mathrm{GO}_{2 n}^{+}(q)=\left\{g \in \mathrm{GL}_{2 n}(q) \mid\left(g^{t}\right) M g=M\right\}
$$

where

$$
M=\left(\begin{array}{cc}
0 & I_{n} \\
I_{n} & 0
\end{array}\right) .
$$

Taking elements of determinant 1, we obtain the special orthogonal group, $\mathrm{SO}_{2 n}^{+}(q)$. This group has a unique subgroup $\Omega_{2 n}^{+}(q)$ of index 2. Quotienting by the center, we obtain the simple projective orthogonal groups, $\mathrm{P} \Omega_{2 n}^{+}(q)$, or $\mathrm{O}_{2 n}^{+}(q)$. By Theorem 11.3.2 of [7], $D_{n}(q) \cong \mathrm{O}_{2 n}^{+}(q)$. 
Finally, we may construct these finite simple groups as the fixed points of an algebraic group:

Set $F$ to be the standard Frobenius endomorphism. Let $\mathcal{G}=\mathrm{SO}_{2 n}(k)$. Then $\mathcal{G}^{F} \cong \mathrm{SO}_{2 n}^{+}(q)$, and as above, this yields $\mathrm{O}_{2 n}^{+}(q)$.

When $n=4$ there are automorphisms of the Dynkin diagram, and hence, we obtain the twisted version ${ }^{3} \mathrm{D}_{4}$. We will describe these groups briefly with the exceptional groups. When $n \geq 4$, there is an order 2 automorphism of the Dynkin diagram, and we obtain another twisted version, ${ }^{2} \mathrm{D}_{n}$. This yields the finite simple groups ${ }^{2} \mathrm{D}_{n}\left(q^{2}\right)$ by setting $K=\mathbb{F}_{q^{2}}$.

We may also construct these finite simple groups as a group of matrices: Let $Q$ be a non-degenerate quadratic form on a vector space $V$ of dimension $2 n$ over $\mathbb{F}_{q}$. Define

$$
f_{Q}(v, w)=(v, w)=Q(v+w)-Q(v)-Q(w)
$$

to be the associated bilinear form. As in [29, Proposition 2.5.3(ii)], we assume that $V$ has a basis $\left\{e_{1}, \ldots, e_{n-1}, f_{1}, \ldots, f_{n-1}, x, y\right\}$ satisfying $Q\left(e_{i}\right)=Q\left(f_{i}\right)=0$, $f_{Q}\left(e_{i}, f_{j}\right)=\delta_{i j}$, and $f_{Q}\left(e_{i}, x\right)=f_{Q}\left(e_{i}, y\right)=f_{Q}\left(f_{i}, x\right)=f_{Q}\left(f_{i}, y\right)=0$ for all $i, j$, and no non-zero vectors $v \in\langle x, y\rangle$ satisfy $Q(v)=0$. (In this case, we say that $V$ has Witt defect 1.) When $q$ is even, the bilinear form degenerates to a symplectic form, and the orthogonal group is the subgroup of the symplectic group, containing the $Q$-isometries. When $q$ is odd, the general orthogonal group is the subgroup of $\mathrm{GL}(V)$ that are $Q$-isometries (i.e. $\{g \in \mathrm{GL}(V) \mid Q(x g)=$ $Q(x), \forall x \in V\})$. Choosing a suitable basis for $V$, we see that (for $q$ odd) this can be identified with

$$
\mathrm{GO}_{2 n}^{-}(q)=\left\{g \in \mathrm{GL}_{2 n}(q) \mid\left(g^{t}\right) N g=N\right\}
$$


where

$$
N=\left(\begin{array}{ccc}
0 & I_{n-1} & 0 \\
I_{n-1} & 0 & 0 \\
0 & 0 & \left(\begin{array}{cc}
2 & 1 \\
1 & 2 \zeta
\end{array}\right)
\end{array}\right)
$$

where $\zeta$ is chosen such that the polynomial $x^{2}+x+\zeta$ is irreducible over $\mathbb{F}_{q}$. Taking elements of determinant 1, we obtain the special orthogonal group, $\mathrm{SO}_{2 n}^{-}(q)$. This group has a unique subgroup $\Omega_{2 n}^{-}(q)$ of index 2. Quotienting by the center, we obtain the simple projective orthogonal groups, $\mathrm{P} \Omega_{2 n}^{-}(q)$, or $\mathrm{O}_{2 n}^{-}(q)$. By Theorem 11.3.2 of [7], ${ }^{2} \mathrm{D}_{n}\left(q^{2}\right) \cong \mathrm{O}_{2 n}^{-}(q)$.

Finally, we may construct these finite simple groups as the fixed points of an algebraic group:

Set $F$ to be the standard Frobenius endomorphism composed with conjugation by the matrix

$$
\left(\begin{array}{ccc}
I_{2 n-2} & 0 & 0 \\
0 & 0 & 1 \\
0 & 1 & 0
\end{array}\right) .
$$

Let $\mathcal{G}=\mathrm{SO}_{2 n}(k)$. Then $\mathcal{G}^{F} \cong \mathrm{SO}_{2 n}^{-}(q)$, and as above, this yields $\mathrm{O}_{2 n}^{-}(q)$.

\subsubsection{The Exceptional Groups of Lie Type}

Unfortunately, the exceptional groups of Lie type do not have nice representations as groups of matrices. Thus, we must view them as the fixed point subgroups of a Frobenius endomorphism acting on an algebraic group, or in terms of the Chevalley groups obtained from the proper Lie algebras, which we describe below.

1. Type $E_{6}$ : In this case, we have an indecomposable root system given by the following Dynkin diagram:

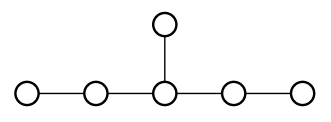


As described above, this yields the finite simple Chevalley group $\mathrm{E}_{6}(q)$ by setting $K=\mathbb{F}_{q}$.

Note that there is an automorphism of order 2 of the Dynkin diagram. Thus, we obtain the twisted version ${ }^{2} \mathrm{E}_{6}$. Setting $K=\mathbb{F}_{q^{2}}$, we obtain the finite simple twisted group ${ }^{2} \mathrm{E}_{6}\left(q^{2}\right)$.

2. Type $E_{7}$ : In this case, we have an indecomposable root system given by the following Dynkin diagram:

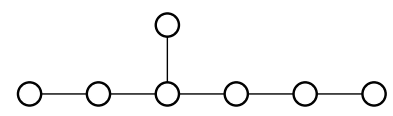

As described above, this yields the finite simple Chevalley group $\mathrm{E}_{7}(q)$ by setting $K=\mathbb{F}_{q}$.

3. Type $E_{8}$ : In this case, we have an indecomposable root system given by the following Dynkin diagram:

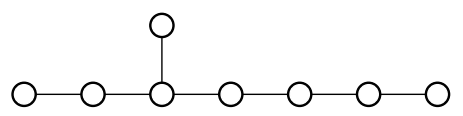

As described above, this yields the finite simple Chevalley group $\mathrm{E}_{8}(q)$ by setting $K=\mathbb{F}_{q}$.

4. Type $F_{4}$ : In this case, we have an indecomposable root system given by the following Dynkin diagram:

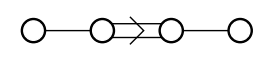

As described above, this yields the finite simple Chevalley group $\mathrm{F}_{4}(q)$ by setting $K=\mathbb{F}_{q}$.

Note that there is an order 2 automorphism of the Dynkin diagram (again, when we ignore the arrow), and this yields the twisted version ${ }^{2} \mathrm{~F}_{4}$. Setting $K=\mathbb{F}_{2^{2 m+1}}$, we obtain the finite simple twisted Ree groups, ${ }^{2} \mathrm{~F}_{4}\left(2^{2 m+1}\right)$. When 
$m=0$, the group is not simple, but its commutator subgroup, the Tits group, ${ }^{2} \mathrm{~F}_{4}(2)^{\prime}$ is simple. The other groups are simple for $m \geq 1$.

5. Type $G_{2}$ : In this case, we have an indecomposable root system given by the following Dynkin diagram:

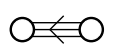

As described above, this yields the finite simple Chevalley group $\mathrm{G}_{2}(q)$ by setting $K=\mathbb{F}_{q}$.

Note that there is an order 2 automorphism of the Dynkin diagram (when we ignore the arrow), and this yields the twisted version ${ }^{2} \mathrm{G}_{2}$. Setting $K=\mathbb{F}_{3^{2 m+1}}$, we obtain the finite simple twisted Ree groups, ${ }^{2} \mathrm{G}_{2}\left(3^{2 m+1}\right)$. When $m=0$, the group is not simple, but its commutator subgroup, ${ }^{2} \mathrm{G}_{2}(3)^{\prime}$, is simple. The other groups are simple for $m \geq 1$.

6. ${ }^{2} \mathrm{~B}_{2}$. As mentioned above, the Dynkin diagram of $\mathrm{B}_{2}$ has an automorphism of order 2 (when we ignore the arrow), and thus, we obtain the twisted version, ${ }^{2} \mathrm{~B}_{2}$. Setting $K=\mathbb{F}_{2^{2 m+1}}$, we obtain the finite simple twisted Suzuki groups, ${ }^{2} \mathrm{~B}_{2}\left(2^{2 m+1}\right)$. When $m=0$, the group is not simple, but the other groups are simple for $m \geq 1$.

7. ${ }^{3} \mathrm{D}_{4}$ : As mentioned above, the Dynkin diagram of $\mathrm{D}_{4}$ has automorphisms of order 3 , and thus, we obtain the twisted version, ${ }^{3} \mathrm{D}_{4}$. Setting $K=\mathbb{F}_{q^{3}}$, we obtain the finite simple twisted groups, ${ }^{3} \mathrm{D}_{4}\left(q^{3}\right)$.

\subsection{Representations of Groups of Lie Type}

Let $\mathbb{F}$ be a field and $G$ be a finite group. An $\mathbb{F}$-representation of $G$ is a homomorphism $\Phi: G \rightarrow \operatorname{GL}(V)$, where $V$ is an $\mathbb{F}$-vector space. If $\operatorname{dim}(V)=n$, then we may fix a basis for $V$ of length $n$, and obtain $\Phi: G \rightarrow \mathrm{GL}_{n}(\mathbb{F})$. Further, such a representation 
$\Phi$ defines an $\mathbb{F} G$-module by taking $V$ as the column space $\mathbb{F}^{n}$ and defining the action of $g \in G$ on a vector $v \in V$ by $g \cdot v:=\Phi(g) v$. Conversely, given any $\mathbb{F} G$-module $V$ of dimension $n$, we obtain a representation $\Phi: G \rightarrow \mathrm{GL}_{n}(\mathbb{F})$ by defining $\Phi(g) v:=g \cdot v$. Thus, we have an identification between $\mathbb{F}$-representations of a finite group $G$ and $\mathbb{F} G$-modules. We say that an $\mathbb{F}$-representation is irreducible if the corresponding $\mathbb{F} G$-module is irreducible. Now, any $\mathbb{F} G$-module $V$ has a composition series

$$
0=V_{0} \subseteq V_{1} \subseteq \cdots \subseteq V_{m}=V
$$

with each factor $V_{i+1} / V_{i}$ irreducible, and these correspond to the irreducible $\mathbb{F}$ representations of $G$. When $\operatorname{char}(\mathbb{F}) \nmid|G|$ and $\mathbb{F}$ is algebraically closed, then the $\mathbb{F}$ representation theory of $G$ is the same as the $\mathbb{C}$-representation theory of $G$ (Maschke's Theorem). However, if $\operatorname{char}(\mathbb{F})|| G \mid$, the $\mathbb{F} G$-modules are no longer necessarily completely reducible, and the representation theory becomes more complicated. We will discuss these representations briefly in Section 2.3.

\subsubsection{Some Character Theoretic Results}

We will rely heavily on results from character theory, many of which are found in [25]. We will discuss the main features of Clifford that are essential in our proofs, and supply proofs that provide helpful insight into various proof techniques. First, we recall the basic definitions of character theory.

Definition 2.2.1. Let $\Phi$ be an $\mathbb{F}$-representation of $G$. Then the $\mathbb{F}$-character, $\chi$, afforded by $\Phi$ is the class function given by $\chi(g):=\operatorname{tr} \circ \Phi(g)$.

The character $\chi$ is said to be irreducible if the representation affording $\chi$ is an irreducible $\mathbb{F}$-representation of $G$. As stated above, when $\operatorname{char}(\mathbb{F}) \nmid|G|$, the $\mathbb{F}$ representation theory of $G$ is the same as the $\mathbb{C}$-representation theory of $G$, and we denote the collection of irreducible complex or ordinary characters of $G$ by $\operatorname{Irr}(G)$. 
Further, if $\psi$ is any ordinary character of $G$, we may decompose $\psi$ as a sum

$$
\psi=\sum_{\chi \in \operatorname{Irr}(G)} a_{\chi} \chi
$$

where each $a_{\chi}$ is a non-negative integer. The following lemmas and theorems are useful when discussing the (ordinary) character theory of subgroups of $G$.

Definition 2.2.2. Let $\chi, \psi$ be class functions on $G$. We denote the restriction of $\chi$ to $H$ by $\chi_{H}$ or $\left.\chi\right|_{H}$.

We define the inner product of $\chi$ and $\psi$ to be

$$
[\chi, \psi]=\frac{1}{|G|} \sum_{g \in G} \chi(g) \overline{\psi(g)}
$$

Notice that for (not necessarily irreducible) characters $\chi$ and $\psi$ of $G$, we have $[\chi, \psi]=$ $[\psi, \chi]$ is a nonnegative integer, and $[\chi, \chi]=1$ iff $\chi \in \operatorname{Irr}(G)$. Now, let $\theta$ be a class function of $H \subseteq G$. Then $\theta^{G}$, the induced class function on $G$, is given by

$$
\theta^{G}(g)=\frac{1}{|H|} \sum_{x \in G, x g x^{-1} \in H} \theta\left(x g x^{-1}\right) .
$$

Lemma 2.2.3 ([25], Theorem 2.18, (Second Orthogonality Relation)). Let $g, h \in G$. Then

$$
\sum_{\chi \in \operatorname{Irr}(G)} \chi(g) \overline{\chi(h)}=0
$$

if $g$ is not conjugate to $h$ in $G$. Otherwise, the sum is equal to $\left|g^{G}\right|$, the size of the conjugacy class of $g$.

Lemma 2.2.4 ([25], Lemma 5.2, (Frobenius Reciprocity)). Let $\theta$ be a class function on $H \subseteq G$, and $\psi$ be a class function on $G$. Then

$$
\left[\theta, \psi_{H}\right]=\left[\theta^{G}, \psi\right]
$$

Proof: By definition, we have

$$
\left[\theta^{G}, \psi\right]=\frac{1}{|G|} \sum_{g \in G} \theta^{G}(g) \overline{\psi(g)}=\frac{1}{|G|} \frac{1}{|H|} \sum_{g \in G} \sum_{x \in G, x g x^{-1} \in H} \theta\left(x g x^{-1}\right) \overline{\psi(g)} .
$$


Now, letting $y=x g x^{-1}$ and observing that $\psi(y)=\psi(g)$, we have

$$
\left[\theta^{G}, \psi\right]=\frac{1}{|G|} \frac{1}{|H|} \sum_{y \in H} \sum_{x \in G} \theta(y) \overline{\psi(y)}=\frac{1}{|H|} \sum_{y \in H} \theta(y) \overline{\psi(y)}=\left[\theta, \psi_{H}\right] .
$$

Theorem 2.2.5 ([25], Theorem 6.2). Let $H \triangleleft G$ and let $\chi \in \operatorname{Irr}(G)$. Let $\theta$ be an irreducible constituent of $\chi_{H}$ and suppose that $\theta=\theta_{1}, \theta_{2}, \ldots, \theta_{t}$ are the distinct conjugates of $\theta$ in $G$. Then

$$
\chi_{H}=e \sum_{i=1}^{t} \theta_{i}
$$

where $e=\left[\theta, \chi_{H}\right]$ is the ramification index.

Proof: For $h \in H$, we have

$$
\theta^{G}(h)=\frac{1}{|H|} \sum_{x \in G, x h x^{-1} \in H} \theta\left(x h x^{-1}\right)=\frac{1}{|H|} \sum_{x \in G} \theta^{x}(h)
$$

since $x h x^{-1} \in H \triangleleft G$ for all $x \in G$. Thus $|H|\left(\theta^{G}\right)_{H}=\sum_{x \in G} \theta^{x}$, and hence, if $\psi \in \operatorname{Irr}(H)$ is not a $G$-conjugate of $\theta$, then $\left[\left(\theta^{G}\right)_{H}, \psi\right]=0$.

Now, by Lemma 2.2.4, as $\theta$ is a constituent of $\chi_{H}, 0 \neq\left[\theta, \chi_{H}\right]=\left[\theta^{G}, \chi\right]$. Hence, $\chi$ is a constituent of $\theta^{G}$. Thus, if $\psi \in \operatorname{Irr}(H)$ is not a $G$-conjugate of $\theta$, then $\left[\chi_{H}, \psi\right]=0$. Thus, every constituent of $\chi_{H}$ must be a $G$-conjugate of $\theta$, hence

$$
\chi_{H}=\sum_{i=1}^{t}\left[\theta_{i}, \chi_{H}\right] \theta_{i}=\sum_{i=1}^{t}\left[\theta, \chi_{H}\right] \theta_{i}=e \sum_{i=1}^{t} \theta_{i} .
$$

Definition 2.2.6. Let $\theta$ be a class function on $H \subseteq G$, and $\psi$ be a class function on G. If $\left[\theta, \psi_{H}\right]>0$, then we will write $\theta \in \psi_{H}$.

Theorem 2.2.7 ([25], Theorem 6.11). (Clifford Correspondence) Let $N \triangleleft G, \theta \in$ $\operatorname{Irr}(N)$, and $T=I_{G}(\theta):=\left\{g \in G \mid \theta^{g}=\theta\right\}$. Let

$$
\mathcal{A}=\left\{\psi \in \operatorname{Irr}(T) \mid \theta \in \psi_{N}\right\}=: \operatorname{Irr}(T \mid \theta) \text { and } \mathcal{B}=\left\{\chi \in \operatorname{Irr}(G) \mid \theta \in \chi_{N}\right\}=: \operatorname{Irr}(G \mid \theta)
$$

Then

(a) If $\psi \in \operatorname{Irr}(T \mid \theta)$, then $\psi^{G} \in \operatorname{Irr}(G \mid \theta)$. 
(b) If $\psi^{G}=\chi \in \mathcal{B}$, then $\left[\psi_{N}, \theta\right]=\left[\chi_{N}, \theta\right]$.

(c) The map $\psi \mapsto \psi^{G}$ is a bijection of $\mathcal{A}$ onto $\mathcal{B}$.

(d) If $T \leq H \leq G$, then the map $\beta \mapsto \beta^{G}$ gives a bijective correspondence $\operatorname{Irr}(H \mid \theta) \rightarrow \operatorname{Irr}(G \mid \theta)$.

Proof: (a) and (b): Let $\chi$ be an irreducible constituent of $\psi^{G}$. Then by Lemma 2.2.4, $\psi$ is an irreducible constituent of $\chi_{T}$. Hence, we have that $\theta \in \psi_{N} \in \chi_{N}$. By Theorem 2.2.5 $\chi_{N}=e \sum_{i=1}^{t} \theta_{i}$, where $\left\{\theta=\theta_{1}, \ldots, \theta_{t}\right\}$ is the set of $G$-conjugates of $\theta$ and $t=[G: T]$. Also by Theorem 2.2.5, $\psi_{N}=f \cdot \theta$ since $\theta$ is the only $T$-conjugate of $\theta$. Further, since $\psi_{N}$ is a constituent of $\chi_{N}, f=\left[\psi_{N}, \theta\right] \leq\left[\chi_{N}, \theta\right]=e$.

Now, we have the chain of inequalities:

$$
e \cdot t \cdot \theta(1)=\chi(1) \leq \psi^{G}(1)=[G: T] \psi(1)=t \cdot f \cdot \theta(1) \leq e \cdot t \cdot \theta(1)
$$

Hence this is a chain of equalities, and thus $e=\left[\chi_{N}, \theta\right]=\left[\psi_{N}, \theta\right]=f$. Finally, we also have $\chi(1)=\psi^{G}(1)$, and as $\chi$ is an irreducible constituent of $\psi^{G}, \chi=\psi^{G} \in \operatorname{Irr}(G \mid \theta)$.

(c) First we show the map is onto. Let $\chi \in \operatorname{Irr}(G \mid \theta)$. Then $\chi_{T}=\sum_{i=1}^{m} \psi_{i}$ for some $\psi_{i} \in \operatorname{Irr}(T)$. As $\theta$ is an irreducible constituent of $\chi_{N}=\sum_{i=1}^{m}\left(\psi_{i}\right)_{N}$, there exists some $\psi_{i} \in \operatorname{Irr}(T)$ with $\theta \in\left(\psi_{i}\right)_{N}$. That is, $\psi_{i} \in \operatorname{Irr}(T \mid \theta)$. By part (a), $\psi_{i}^{G} \in \operatorname{Irr}(G \mid \theta)$. Then, by Lemma 2.2.4, $0 \neq\left[\chi_{T}, \psi_{i}\right]=\left[\chi, \psi_{i}^{G}\right]$. Therefore $\chi=\psi_{i}^{G}$, as both are irreducible.

Next, we show that the map is one to one. Suppose by way of contradiction that $\psi, \psi^{\prime} \in \operatorname{Irr}(T \mid \theta)$ with $\psi^{G}=\chi=\psi^{\prime G}$. Then $\psi, \psi^{\prime} \in \chi_{T}$, hence $\psi+\psi^{\prime} \in \chi_{T}$. Thus

$$
\left[\psi_{N}, \theta\right]=\left[\chi_{N}, \theta\right] \geq\left[\left(\psi+\psi^{\prime}\right)_{N}, \theta\right]=\left[\psi_{N}, \theta\right]+\left[\psi_{N}^{\prime}, \theta\right]
$$

However, this implies $\left[\psi_{N}^{\prime}, \theta\right] \leq 0$, a contradiction. Thus, the map is one to one.

(d) Finally, we observe that $\operatorname{Ind}_{H}^{G}=\operatorname{Ind}_{T}^{G} \circ\left(\operatorname{Ind}_{T}^{H}\right)^{-1}$ is a composition of bijections, and is hence a bijection. 


\subsubsection{Fields of Values of Characters}

Let $\chi \in \operatorname{Irr}(G)$ be an irreducible character of a finite group $G$. We define the field of values of $\chi$ to be

$$
\mathbb{Q}(\chi):=\mathbb{Q}(\{\chi(g) \mid g \in G\}) .
$$

There are many results concerning groups whose characters are real-valued, i.e. $\mathbb{Q}(\chi) \subseteq$ $\mathbb{R}$ for all $\chi \in \operatorname{Irr}(G)$. We will provide some of these results, as the proof techniques are helpful in proving our results on $m$-rational groups.

First, we define the determinantal order of a character. Let $\chi \in \operatorname{Irr}(G)$ be afforded by $\Phi: G \rightarrow \mathrm{GL}_{n}(\mathbb{C})$. Then we may post-compose $\Phi$ with the determinant function, and we obtain a linear character $\operatorname{det}(\chi):=\operatorname{det} \circ \Phi \in \operatorname{Irr}(G)$. As the set of linear irreducible characters form a group under multiplication, we may define $o(\chi)$ to be the order (in the usual group theoretic sense) of $\operatorname{det}(\chi)$ in the group of linear characters of $G$.

Lemma 2.2.8 ([40], Lemma 2.1). Suppose that $N \triangleleft G$ has odd index. If $\theta \in \operatorname{Irr}(N)$ is $G$-invariant and real, then $\theta$ has a unique real extension $\eta$ to $G$. Furthermore, $o(\eta)=o(\theta)$.

Proof: We proceed by induction on $[G: N]$. First, we prove the extendibility of $\theta$. If $\theta$ is not extendible to $G$, then by Corollary 11.31 of [25], there exists some subgroup $P$ such that $P / N$ is a Sylow subgroup of $G / N$ and $\theta$ is not extendible to $P$. Thus it suffices to show that $\theta$ is extendible to $G$ for $G / N$ a $p$-group. Hence, we assume that $G / N$ is a $p$-group. Then there exists a subgroup $M / N \triangleleft G / N$ with $[G / N: M / N]=[G: M]=p$. By the induction hypothesis, as $[M: N]<[G: N]$,

$\theta$ has a unique real extension, $\delta$, to $M$. Now, it is clear that $\delta^{g} \in \operatorname{Irr}(M)$ is also a real extension of $\theta$ for any $g \in G$ since $\delta^{g}(n)=\delta\left(g n g^{-1}\right)=\theta\left(g n g^{-1}\right)=\theta^{g}(n)=\theta(n)$ for any $n \in N$. Thus by uniqueness, $\delta^{g}=\delta$ for any $g \in G$. That is, $\delta$ is $G$-invariant. By Corollary 6.20 of [25], $\delta$ is extendible to $G$. Let $\chi$ be an extension of $\delta$. Then 
$\chi_{N}=\left(\chi_{M}\right)_{N}=\delta_{N}=\theta$. That is, $\chi$ is an extension of $\theta$.

Now, by Gallagher's Theorem [25, Theorem 6.16], the map $\lambda \mapsto \lambda \chi$ is a bijection between $\operatorname{Irr}(G / N)$ and the set of irreducible constituents of $\theta^{G}$. It follows that the restriction of this map to the set of linear irreducible characters of $G / N$ gives a bijection onto the set of extensions of $\theta$ to $G$. Since $[G: N]$ is odd and the number of linear characters of $G / N$ is equal to $\left[G / N:(G / N)^{\prime}\right]$, there is an odd number of extensions of $\theta$ to $G$. Since complex conjugation acts on the set of extensions of $\theta$ to $G$, there must be at least one real extension $\eta:=\beta \chi \in \operatorname{Irr}(G)$ of $\theta$. Suppose that $\psi$ is another real extension of $\theta$. Then there exists a unique (since the mapping is a bijection) linear $\lambda \in \operatorname{Irr}(G / N)$ such that $\lambda \eta=\psi$. As $\psi$ is real valued, we have $\bar{\psi}=\overline{\lambda \eta}=\bar{\lambda} \eta=\lambda \eta=\psi$. Thus $\bar{\lambda}=\lambda$ by uniqueness, hence $\lambda$ is real valued. However, it is easy to see that any real-valued linear character must satisfy $\lambda^{2}=1$ : Since the set of linear characters form a group under multiplication, there exists some $n \in \mathbb{N}$ such that $\lambda^{n}=1$. Thus, $\lambda(g)$ is an $n$-th root of unity for all $g \in G$. As $\lambda(g)$ is real valued, it must be the case that $n \leq 2$. Thus $\lambda^{2}=1$. As stated above, there are $\left[G / N:(G / N)^{\prime}\right]$ linear characters of $G / N$, and thus the order of $\lambda$ in this group must divide $\left[G / N:(G / N)^{\prime}\right]$, which is odd. Therefore, we must have $\lambda=1$. That is, $\psi=\eta$, and $\theta$ has a unique real extension $\eta$ to $G$.

Finally, we show that $o(\eta)=o(\theta)$. Let $\nu=\operatorname{det}(\theta)$ and $\mu=\operatorname{det}(\eta)$. Then, $\mu_{N}=(\operatorname{det}(\eta))_{N}=\operatorname{det}\left(\eta_{N}\right)=\operatorname{det}(\theta)=\nu$. Thus, $o(\nu) \leq o(\mu)$. Now, $\theta$ and $\eta$ are real, and thus, $\operatorname{det}(\theta)=\nu$ and $\operatorname{det}(\eta)=\mu$ are real. (This follows from theorem 4.14 of [25] and Schur's Lemma). As shown in the previous paragraph, every real-valued linear character $\lambda$ satifies $\lambda^{2}=1$, so $o(\mu)$ and $o(\nu)$ must therefore divide 2. If $o(\nu)=2$, then $o(\mu)=2$ and we are done. If $\nu=1$, then $\mu_{N}=1$, so we may view $\mu$ as an irreducible linear character of $G / N$. However, $G / N$ has odd order, and therefore the group of linear characters of $G / N$ must have odd order. Thus, the order of $\mu$ must be odd and at most 2 , and hence $\mu$ is trivial in $G / N$. By inflation, $\mu$ is trivial in $G$. Thus $o(\eta)=o(\theta)$. 
Theorem 2.2.9 ([40], Theorem 2.3). Let $N \triangleleft G$, and let $\theta \in \operatorname{Irr}(N)$ be $G$-invariant, real of odd degree. Suppose that $o(\theta)=1$. Then $\theta$ has a unique real extension $\eta \in \operatorname{Irr}(G)$ such that $o(\eta)=1$.

Proof: First, we prove that $\theta$ extends to $G$. By Corollary 11.31 of [25], it suffices to show that $\theta$ extends to $P$, where $P / N$ is a Sylow $p$-subgroup of $G / N$. If $p=2$, then by Corollary 6.28 of [25], $\theta$ has a unique extension $\chi \in \operatorname{Irr}(P)$ with $([P: N], o(\chi))=1$, since $([P: N], o(\theta) \theta(1))=1$. If $p$ is odd, then by Lemma 2.2.8, $\theta$ has a unique real extension to $P$. Therefore, in either case, $\theta$ is extendible to $P$, and hence to $G$. Let $\chi \in \operatorname{Irr}(G)$ be any extension of $\theta$ to $G$. As shown in Lemma 2.2.8 (using Gallagher's theorem), the set of extensions of $\theta$ to $G$ is $\{\lambda \chi \mid \lambda \in \operatorname{Irr}(G / N), \lambda(1)=1\}=\{\lambda \chi \mid \lambda \in \operatorname{Irr}(G / M)\}$, where $M=G^{\prime} N \triangleleft G$.

To prove uniqueness, we proceed by induction on $[G: N]$. First, we assume $G=M=G^{\prime} N$. Then $G / M=1$, and thus $\chi$ is the unique extension of $\theta$ to $G$. Since $(\bar{\chi})_{N}=\overline{\chi_{N}}=\bar{\theta}=\theta, \bar{\chi}$ is also an extension of $\theta$. By uniqueness, $\chi=\bar{\chi}$, and $\chi$ is real. Further, we see that $\operatorname{det}(\chi)_{N}=\operatorname{det}\left(\chi_{N}\right)=\operatorname{det}(\theta)=1 \in \operatorname{Irr}(N)$, and thus, as $G=M$, $\operatorname{det}(\chi)$ is the unique extension of $1_{N}$ to $G$. Of course, $1_{G}$ extends $1_{N}$, and therefore $\operatorname{det}(\chi)=1$.

Now, assume $M<G$. By induction, $\theta$ has a unique real extension $\nu \in \operatorname{Irr}(M)$ with $o(\nu)=1$. Now, by uniqueness, we have that $\nu$ is $G$-invariant since $\nu^{g}$ is also a real extension of $\theta$ with $o\left(\nu^{g}\right)=1$ for any $g \in G$. Further, we have $\nu(1)=\theta(1)$, which is odd. Thus, if $N<M$, then again by the inductive hypothesis, there exists a unique real extension $\tau$ of $\nu$ to $G$ with $o(\tau)=1$. Thus, in this case, we have $\tau$ is a real extension of $\theta$ to $G$ with $o(\tau)=1$. Suppose that $\chi \in \operatorname{Irr}(G)$ is another real extension of $\theta$ with $\operatorname{det}(\chi)=1$. Then $\chi_{M}$ is a real extensions of $\theta$ to $M$ and $(\operatorname{det}(\chi))_{M}=\operatorname{det}\left(\chi_{M}\right)=1$. By the uniqueness statement in the inductive hypothesis, we have $\chi_{M}=\nu$. Then, as $\chi$ is an extension of $\chi_{M}=\nu$, again by the uniqueness statement in the inductive hypothesis, $\chi=\tau$. 
Therefore, we may assume that $M=N$. Thus $G^{\prime} \leq N$, and $G / N$ is abelian. Let $P / N$ be a Sylow 2-subgroup. Since $G / N$ is abelian, $P / N \triangleleft G / N$, and as $P / N$ is a Sylow subgroup, $(|P / N|,[G / N: P / N])=1$. Thus, by the Schur-Zassenhaus theorem, $P / N$ has a complement $H / N$ in $G / N$. Notice that $|H / N|$ is odd, and thus by Lemma 2.2.8, $\theta$ has a unique real extension $\xi$ to $H$ with $o(\xi)=o(\theta)=1$. Also, by Corollary 6.28 of [25], there is a unique extension $\hat{\theta} \in \operatorname{Irr}(P)$ of $\theta$, and furthermore, $o(\hat{\theta})=o(\theta)=1$. As usual, $\overline{\hat{\theta}}$ and $\hat{\theta}^{g}$ are also extensions of $\theta$ for any $g \in G$, so by uniqueness, $\hat{\theta}$ is real and $G$-invariant.

Now, we claim that restriction defines a bijection $\operatorname{Irr}(G \mid \hat{\theta}) \rightarrow \operatorname{Irr}(H \mid \theta)$. By Gallagher's Theorem, we know that if $\chi \in \operatorname{Irr}(G \mid \hat{\theta})$ (that is, if $\chi \in \operatorname{Irr}(G)$ satisfies $\left.\left[\chi_{P}, \hat{\theta}\right] \neq 0\right)$, then every other character in $\operatorname{Irr}(G \mid \hat{\theta})$ is of the form $\beta \chi$ for some $\beta \in G / P$. Likewise, if $\psi \in \operatorname{Irr}(H \mid \theta)$, then every other character in $\operatorname{Irr}(H \mid \theta)$ is of the form $\beta^{\prime} \psi$ for some $\beta^{\prime} \in H / N$. First, we show that if $\chi \in \operatorname{Irr}(G \mid \hat{\theta})$ and $\psi \in \operatorname{Irr}(H \mid \theta)$ with $\left[\chi_{H}, \psi\right]=\left[\chi, \psi^{G}\right] \neq 0$, then $\left[\chi_{H}, \psi\right]=1$, from which it follows that $\chi_{H}=\psi$ since $\chi_{H}(1)=\chi(1)=\hat{\theta}(1)=\theta(1)=\psi(1)$. Then it will suffice to show that given $\chi \in \operatorname{Irr}(G \mid \hat{\theta})$, there exists some $\psi \in \operatorname{Irr}(H \mid \theta)$ with $\left[\chi_{H}, \psi\right]>0$, and given some $\psi \in \operatorname{Irr}(H \mid \theta)$, there exists $\chi \in \operatorname{Irr}(G \mid \hat{\theta})$ with $\left[\chi_{H}, \psi\right]>0$. Suppose $\chi \in \operatorname{Irr}(G \mid \hat{\theta})$, $\psi \in \operatorname{Irr}(H \mid \theta)$, and $\left[\chi_{H}, \psi\right]>0$. Then by Theorem 2.2.5, we may write $\chi_{P}=e \hat{\theta}$ and $\psi_{N}=f \theta$, since $\hat{\theta}$ is $G$-invariant and $\theta$ is $H$-invariant (Recall that $\theta$ is $G$-invariant by assumption). Then

$$
f=\left[\psi_{N}, \theta\right] \leq\left[\left(\chi_{H}\right)_{N}, \theta\right]=\left[\left(\chi_{P}\right)_{N}, \theta\right]=e\left[\hat{\theta}_{N}, \theta\right]=e
$$

Further, we see that

$$
e=\left[\chi_{P}, \hat{\theta}\right] \leq\left[\left(\psi^{G}\right)_{P}, \hat{\theta}\right]=\left[\left(\psi_{N}\right)^{P}, \hat{\theta}\right]=f\left[\theta^{P}, \hat{\theta}\right]=f\left[\theta, \hat{\theta}_{N}\right]=f,
$$

where the second equality follows from Mackey's formula. Therefore these must be equalities and $\left[\left(\chi_{H}\right)_{N}, \theta\right]=\left[\psi_{N}, \theta\right]$. Thus $\left[\chi_{H}, \psi\right]=1$. Finally, we make the following observations: Given $\chi \in \operatorname{Irr}(G \mid \hat{\theta}),\left[\left(\chi_{H}\right)_{N}, \theta\right]=\left[\left(\chi_{P}\right)_{N}, \theta\right]>0$, so there exists some 
$\psi \in \operatorname{Irr}(H \mid \theta)$ with $\left[\chi_{H}, \psi\right]>0$. Also, given $\psi \in \operatorname{Irr}(H \mid \theta),\left[\left(\psi^{G}\right)_{P}, \hat{\theta}\right]=\left[\left(\psi_{N}\right)^{P}, \hat{\theta}\right]>0$, again by Mackey's formula, so there exists $\chi \in \operatorname{Irr}(G \mid \hat{\theta})$ with $\left[\chi, \psi^{G}\right]=\left[\chi_{H}, \psi\right]>0$, as desired.

Therefore, there exists a unique $\rho \in \operatorname{Irr}(G \mid \hat{\theta})$ such that $\rho_{H}=\xi$. Thus $\rho_{N}=$ $\left(\rho_{H}\right)_{N}=\xi_{N}=\theta$, and $\rho$ is an extension of $\theta$. Since $\xi$ is real, $\bar{\rho}_{H}=\xi$, and the restriction mapping is one to one, it follows that $\bar{\rho}=\rho$, and $\rho$ is real. Now, observe that $\operatorname{det}(\rho)_{P}=\operatorname{det}\left(\rho_{P}\right)=\operatorname{det}(\hat{\theta})=1$ and $\operatorname{det}(\rho)_{H}=\operatorname{det}\left(\rho_{H}\right)=\operatorname{det}(\xi)=1$. Thus, as $G / N=P / N \times H / N$, it follows that $\operatorname{det}(\rho)=1 \operatorname{since} \operatorname{det}(\rho)$ is a homomorphism. Finally, suppose $\mu \in \operatorname{Irr}(G)$ is real, extends $\theta$ and satisfies $o(\mu)=1$. Then $\mu_{P}$ is a real extension of $\theta$ with $o\left(\mu_{P}\right)=1$. By uniqueness, we have $\mu_{P}=\hat{\theta}=\rho_{P}$. Further, $\mu_{H}$ is a real extension of $\theta$, and since the restriction map is a bijection, as above, we have $\mu_{H}=\xi=\rho_{H}$. Thus, $\mu=\rho$, and uniqueness follows.

Theorem 2.2.10 ([39], Theorem 2.1). Suppose that $N \triangleleft G$, and let $\theta \in \operatorname{Irr}(N)$ be real of odd degree. Suppose that $o(\theta)=1$. If $T=I_{G}(\theta):=\left\{g \in G \mid \theta^{g}=\theta\right\}$, then $\theta$ has a unique real extension $\gamma \in \operatorname{Irr}(T)$ such that $o(\gamma)=1$. Furthermore, $\mathbb{Q}(\gamma)=\mathbb{Q}(\theta)$. Hence, $\chi=\gamma^{G} \in \operatorname{Irr}(G)$ is an irreducible character of $G$ over $\theta$ with values in $\mathbb{Q}(\theta)$.

Proof. The first part is exactly Theorem 2.2.9 with $T=G$. We now show that $\mathbb{Q}(\gamma)=\mathbb{Q}(\theta)$. Since $\gamma$ is an extension of $\theta, \gamma_{N}=\theta$, and thus $\mathbb{Q}(\theta) \subseteq \mathbb{Q}(\gamma)$. Let $\sigma$ be a Galois automorphism of $\mathbb{Q}(\gamma)$ fixing everything in $\mathbb{Q}(\theta)$. We claim that $\sigma$ also fixes $\gamma$, giving us the desired equality. Since $\mathbb{Q}(\gamma) \subseteq \mathbb{R}$, we have that $\gamma^{\sigma}$ is a real valued character. Further, $\gamma^{\sigma}(n)=\sigma(\gamma(n))=\sigma(\theta(n))=\theta(n)$ for all $n \in N$, and thus, $\gamma^{\sigma}$ is an extension of $\theta$. Finally, we see that for a representation $\Phi$ affording $\gamma$, $\operatorname{det}(\sigma(\Phi(g)))=\sigma(\operatorname{det}(\Phi(g)))$ for all $g \in T$, and thus $\operatorname{det}\left(\gamma^{\sigma}\right)=\sigma(\operatorname{det}(\gamma))=\sigma(1)=1$. Thus $o\left(\gamma^{\sigma}\right)=1$. By uniqueness of the extensions, it follows that $\gamma^{\sigma}=\gamma$, and $\mathbb{Q}(\theta)=\mathbb{Q}(\gamma)$. The last part is an immediate consequence of Theorem 2.2.7 and the formula for the induced character. 


\subsubsection{Some Deligne-Lusztig Theory}

In this thesis, we are primarily concerned with the semisimple Deligne-Lusztig characters. Further, in the modular version of our main problem, we will use results dealing with the Lusztig series. We will discuss the construction of these characters, as well as reference important facts regarding the Lusztig series. We follow [6], [10], and [33] in this short exposé.

Let $\mathcal{G}$ be a connected reductive algebraic group over $k=\overline{\mathbb{F}}_{q}$, and let $F$ be a Frobenius endomorphism of $\mathcal{G}, G:=\mathcal{G}^{F}$. A torus $\mathcal{T}$ of $\mathcal{G}$ is a finite direct product of copies of the multiplicative group $\mathbb{G}_{m}, \mathbb{G}_{m}(k) \cong k^{\times}$. Two connected reductive algebraic groups $\mathcal{G}$ and $\mathcal{G}^{*}$ are said to be dual if there exist maximal tori $\mathcal{T}$ and $\mathcal{T}^{*}$ of $\mathcal{G}$ and $\mathcal{G}^{*}$, respectively, and an isomorphism from the character group of $\mathcal{T}$ to the cocharacter group of $\mathcal{T}^{*}$, sending the roots of $\mathcal{G}$ to the coroots of $\mathcal{G}^{*}$. Fix an $F$-stable maximal torus $\mathcal{T}$, and $\mathcal{B}$ a Borel containing $\mathcal{T}$. Then $\mathcal{B}=\mathcal{U} \mathcal{T}$, where $\mathcal{U}$ is the unipotent radical of $\mathcal{B}$. Then, for every irreducible character $\theta \in \mathcal{T}^{F}=: T$, we can define a virtual character $R_{T}(\theta)$. We follow the notation used in [6, Section 7.2]: First, let $\tilde{X}$ be the preimage of $\mathcal{U}$ under the Lang map $L$ (recall $L: g \mapsto g^{-1} F(g)$ ), and $\overline{\mathbb{Q}}_{p}$ the algebraic closure of the $p$-adic numbers. Write $H_{c}^{i}\left(\tilde{X}, \overline{\mathbb{Q}}_{p}\right)$ to denote the $i$-th $p$-adic cohomology group of $\tilde{X}$ with compact support, and $H_{c}^{i}\left(\tilde{X}, \overline{\mathbb{Q}}_{p}\right)_{\theta}$ to denote the $T$-submodule of $H_{c}^{i}\left(\tilde{X}, \overline{\mathbb{Q}}_{p}\right)$ on which $T$ acts by $\theta$. Then

$$
R_{T}(\theta)(g):=\sum_{i \geq 0}(-1)^{i} \operatorname{trace}\left(g, H_{c}^{i}\left(\tilde{X}, \overline{\mathbb{Q}}_{p}\right)_{\theta}\right) .
$$

Definition 2.2.11. Let $\mathcal{T}$ and $\mathcal{T}^{\prime}$ be maximal tori of the algebraic group $\mathcal{G}$, with Frobenius morphism $F$, and let $T:=\mathcal{T}^{F}$ and $T^{\prime}=\mathcal{T}^{\prime F}$. Assume $\theta \in \operatorname{Irr}\left(\mathcal{T}^{F}\right)$ and $\theta^{\prime} \in \operatorname{Irr}\left(\mathcal{T}^{F}\right)$. The pairs $(T, \theta)$ and $\left(T^{\prime}, \theta^{\prime}\right)$ are called geometrically conjugate if there exists an element $g \in \mathcal{G}$ which conjugates $\mathcal{T}$ to $\mathcal{T}^{\prime}$ and for any $n$ satisfying $g \in \mathcal{G}^{F^{n}}$, we have $\theta \circ N_{F^{n} / F}=\theta^{\prime} \circ N_{F^{n} / F^{\circ}}$ ad $g$, where $N_{F^{n} / F}$ is the norm map $N_{F^{n} / F}: \mathcal{T} \rightarrow \mathcal{T}: \tau \mapsto \tau \cdot F(\tau) \cdots F^{n-1}(\tau)$ and ad $g$ is the functor on representations 
induced by conjugation by $g$.

Theorem 2.2.12. [6, Theorem 7.3.8] $R_{T}(\theta)$ and $R_{T^{\prime}}\left(\theta^{\prime}\right)$ share an irreducible constituent if and only if the pairs $(T, \theta)$ and $\left(T^{\prime}, \theta^{\prime}\right)$ are geometrically conjugate.

Further, every irreducible character $\chi \in \operatorname{Irr}(G)$ occurs as a constituent of some $R_{T}(\theta)$, and therefore, these geometric conjugacy classes partition $\operatorname{Irr}(G)$ into a disjoint union of Lusztig series [33, Section 5.1]. Recall that an element $s \in \mathcal{G}$ is called semisimple if $s$ is diagonalizable. Equivalently, $s$ is semisimple if $s$ is a $p^{\prime}$-element. An element $u$ is unipotent if $u-1$ is nilpotent. For any element $g \in \mathcal{G}$, there is a (unique) Jordan decomposition $g=s u=u s$ into a semisimple part and a unipotent part. Finally, a regular element $g$ has $C_{\mathcal{G}}(g)$ with dimension equal to that of the rank of $\mathcal{G}$. In particular, the connected component of the centralizer of a regular semisimple element is a maximal torus in $\mathcal{G}$.

Theorem 2.2.13 (Theorem 5.1, [33]). Let $\mathcal{G}$ be as above, with Frobenius endomorphism $F$. Let $s \in G:=\mathcal{G}^{F}$ be semisimple.

1. If $\chi(s) \neq 0$ for some $\chi \in \operatorname{Irr}(G)$, then there exist geometrically conjugate pairs $(T, \theta)$ and $\left(T^{\prime}, \theta^{\prime}\right)$ with $R_{T}(\theta)(s) \neq 0$ and $\left[\chi, R_{T^{\prime}}\left(\theta^{\prime}\right)\right] \neq 0$.

2. If $R_{T}(\theta)(s) \neq 0$, then there exists $g \in G$ with $\mathcal{T}^{g} \leq C_{\mathcal{G}}(s)$.

Further, there is a formula for the degree of $R_{T}(\theta)$ and the value of $R_{T}(\theta)$ on semisimple elements, [6, Proposition 7.5.3]:

$$
\begin{gathered}
R_{T}(\theta)(1)=\epsilon_{\mathcal{G}} \epsilon_{\mathcal{T}}[G: T]_{p^{\prime}}, \text { and } \\
R_{T}(\theta)(s)=\frac{\epsilon_{\mathcal{T}} \epsilon_{C^{\circ}(s)}}{|T|\left|C^{\circ}(s)^{F}\right|_{p}} \sum_{g \in G, g^{-1} s g \in T} \theta\left(g^{-1} s g\right),
\end{gathered}
$$

where $\epsilon_{X}=(-1)^{\text {relative rank of } X}$. We note also that $R_{T}(\theta)(u)=1$ for any regular unipotent element $u \in G$, [10, Lemma 14.45].

Typically, the characters $\pm R_{T}(\theta)$ will be irreducible: 
Theorem 2.2.14. [6, Corollary 7.3.5] If $\theta$ is an irreducible character in general position (i.e. the Weyl group $W(T)$ acts on $\theta$ with $\operatorname{Stab}_{W(T)}(\theta)=1$ ), then $\pm R_{T}(\theta)$ is an irreducible character.

Note that $\theta=1$ is not in general position, and thus $R_{T}(1)$ is reducible. The irreducible constituents of $R_{T}(1)$ are called the unipotent characters of $G$. For a geometric conjugacy class $\mathcal{K}$, we define the semisimple character associated to $\mathcal{K}$ by

$$
\chi_{\mathcal{K}}^{s s}:= \pm \sum_{(T, \theta) \in \mathcal{K}} \frac{R_{T}(\theta)}{\left[R_{T}(\theta), R_{T}(\theta)\right]} .
$$

Theorem 2.2.15. [10, Theorem 13.13] The $G$-conjugacy classes of pairs $(\mathcal{T}, \theta)$ are in one-to-one correspondence with the $G^{*}=\mathcal{G}^{* F}$-conjugacy classes of pairs $\left(\mathcal{T}^{*}, s\right)$, where $s$ is semisimple in $G^{*}$ and $\mathcal{T}^{*}$ is a maximal torus containing $s$.

Using this correspondence, we are able to redefine the rational Lusztig series, now in terms of the $G^{*}$-conjugacy classes of semisimple elements of the dual group:

Definition 2.2.16. A rational Lusztig series $\mathcal{E}(G,(s))$ associated to the $G^{*}$-conjugacy class $(s)$ of a semisimple element $s \in G^{*}$ is the set of irreducible characters of $G$ which occur as a constituent of some $R_{T}(\theta)$, where $(\mathcal{T}, \theta)$ is the $G^{*}$-conjugacy class associated to $(s)$, as stated above.

Thus, as mentioned above, the rational Lusztig series (from now on, we will refer to this as, simply, a rational series) associated to particular $G^{*}$-conjugacy classes of semisimple elements of $G^{*}$ form a partition of $\operatorname{Irr}(G)$, and $\mathcal{E}(G,(1))$ are the unipotent characters of $G$. If $s \in G^{*}$ is a semisimple regular element and $\mathcal{G}$ has connected center, then the semisimple character associated to the class $(s)$ is irreducible, and we will denote this by $\chi_{s} \in \operatorname{Irr}(G)$. More generally, this is true if $s$ has connected centralizer. In this case, by Corollary 14.47 of [10],

$$
\chi_{s}(1)=\left[G^{*}: C_{G^{*}}(s)\right]_{p^{\prime}} .
$$


Theorem 2.2.17. [10, Theorem 13.23] Let $\mathcal{G}$ be a connected reductive group. For any semisimple element $s \in G^{*}$, there exists a bijection

$$
\mathcal{E}(G,(s)) \rightarrow \mathcal{E}\left(C_{\mathcal{G}^{*}}(s)^{F}, 1\right)
$$

and for any $\chi \in \mathcal{E}(G,(s))$, we have

$$
\chi(1)=\frac{|G|_{p^{\prime}}}{\left|C_{\mathcal{G}^{*}}(s)^{F}\right|_{p^{\prime}}} \psi_{s}(\chi)(1),
$$

where $\psi_{s}(\chi)$ is the image of $\chi$ under this bijection.

As it turns out, the block theory related to the rational series proves to be extremely fruitful, and thus, we will give a description of modular representation theory, and state the important results from Deligne-Lusztig theory associated to blocks.

\subsection{Modular Character Theory}

In this section, we discuss the basics of modular representation theory, blocks, and the Deligne-Lusztig theory associated to blocks. We will follow the notation of [37]. For the remainder of this section, $G$ is a finite group, and $\ell$ is a prime dividing the order of $G$. Let $R$ be the ring of algebraic integers in $\mathbb{C}$, and choose $M$ to be a maximal ideal of $R$ containing $\ell R$ so that $\mathbb{F}=R / M$ is an algebraically closed field of characteristic $\ell$. Define $*: R \rightarrow \mathbb{F}$ to be the natural quotient map. Set

$$
U=\left\{\xi \in \mathbb{C} \mid \xi^{m}=1 \text { for some } m \text { not divisible by } \ell\right\} \subseteq R \text {. }
$$

Then $*: U \rightarrow \mathbb{F}^{\times}$is an isomorphism of multiplicative groups [37, Lemma 2.1]. Let $G^{\circ}$ denote the set of $\ell$-regular elements of $G$. Let $\Phi: G \rightarrow \mathrm{GL}_{n}(\mathbb{F})$ be an $\mathbb{F}$-representation of $G$. Then, for any $g \in G^{\circ}$, we have that all eigenvalues of $\Phi(g)$ (which necessarily lie in $\left.\mathbb{F}^{\times}\right)$are of the form $\xi_{1}^{*}, \ldots, \xi_{n}^{*}$, for some unique $\xi_{1}, \ldots, \xi_{n} \in U$. Then the $\ell$-Brauer character of $G$ afforded by $\Phi$ is the class function on $G^{\circ}$,

$$
\phi: G^{\circ} \rightarrow \mathbb{C}
$$


defined by

$$
\phi(g)=\xi_{1}+\cdots+\xi_{n}
$$

As before, if $\Phi$ is an irreducible representation of $G$, then $\phi$ is an irreducible $\ell$-Brauer character of $G$, and we call the collection of irreducible $\ell$-Brauer characters $\operatorname{IBr}_{\ell}(G)$. Further, $\operatorname{IBr}_{\ell}(G)$ is a basis for the space of class functions of $G^{\circ}$, and $\left|\operatorname{IBr}_{\ell}(G)\right|$ is equal to the number of conjugacy classes of $\ell$-regular elements.

Now, for any ordinary irreducible character $\chi \in \operatorname{Irr}(G)$, the restriction of $\chi$ to the $\ell$-regular classes of $G, \chi^{\circ}$, is a class function of $G^{\circ}$, and is therefore an integral combination of the irreducible $\ell$-Brauer characters:

$$
\chi^{\circ}=\sum_{\phi \in \operatorname{IBr}_{\ell}(G)} d_{\chi \phi} \phi,
$$

and we obtain the $|\operatorname{Irr}(G)| \times\left|\operatorname{IBr}_{\ell}(G)\right|$ decomposition matrix,

$$
D=\left(d_{\chi \phi}\right)_{\chi \in \operatorname{Irr}(G), \phi \in \operatorname{IBr}_{\ell}(G)}
$$

\subsubsection{Blocks}

For $\chi \in \operatorname{Irr}(G)$, we have the associated algebra homomorphism $\omega_{\chi}: Z(\mathbb{C} G) \rightarrow \mathbb{C}$ which is evaluated on a conjugacy class sum as

$$
\omega_{\chi}\left(\sum_{x \in \mathcal{K}} x\right)=\frac{|\mathcal{K}| \chi\left(x_{\mathcal{K}}\right)}{\chi(1)},
$$

where $x_{\mathcal{K}}$ is a representative of the class $\mathcal{K}$. Note that this is an algebraic integer, [32, Corollary 2.3.3]. We define

$$
\lambda_{\chi}\left(\sum_{x \in \mathcal{K}} x\right)=\omega_{\chi}\left(\sum_{x \in \mathcal{K}} x\right)^{*},
$$

where $*$ is the map defined above. Likewise, for $\phi \in \operatorname{IBr}_{\ell}(G)$ afforded by $\Phi$, an F-representation of $G$, we may define

$$
\lambda_{\phi}\left(\sum_{x \in \mathcal{K}} x\right) I_{n}=\Phi\left(\sum_{x \in \mathcal{K}} x\right) .
$$


The $\ell$-blocks $B$ of $G$ are the equivalence classes of $\operatorname{Irr}(G) \cup \operatorname{IBr}_{\ell}(G)$ subject to the relation $\chi \sim \phi$ if $\lambda_{\chi}=\lambda_{\phi}$.

Theorem 2.3.1 ([37], Theorem 3.3). If $\chi \in \operatorname{Irr}(G)$ and $\phi \in \operatorname{IBr}_{\ell}(G)$ are such that $d_{\chi \phi} \neq 0$, then $\chi$ and $\phi$ lie in the same $\ell$-block B. Consequently,

$$
\operatorname{IBr}_{\ell}(B)=\left\{\phi \in \operatorname{IBr}_{\ell}(G) \mid d_{\chi \phi} \neq 0 \text {, for some } \chi \in \operatorname{Irr}(B)\right\}
$$

Now, we return to the Deligne-Lusztig theory of blocks. Let $\chi \in \operatorname{Irr}(G)$, and suppose that $\chi$ belongs to the rational series $\mathcal{E}(G,(s))$ labeled by the semisimple element $s$. We may decompose $s$ into an $\ell$-part and an $\ell^{\prime}$-part, say $s=u t=t u$, where $\ell \nmid|t|$. Define

$$
\mathcal{E}_{\ell}(G,(t)):=\bigcup \mathcal{E}(G,(v t)),
$$

where the union is taken over all $\ell$-elements, $v$, of $C_{G^{*}}(t)$. Clearly, $\chi \in \mathcal{E}_{\ell}(G,(t))$. By a result of Broué and Michel, $\mathcal{E}_{\ell}(G,(t))$ is a union of $\ell$-blocks, [4, Theorem 2.2]. In particular, we may say $\chi \in \operatorname{Irr}(B)$ for some block $B$.

Definition 2.3.2. Let $a$ be an integer and $p$ be a prime. Then $(a)_{p}$ denotes the p-part of $a$, the highest power of $p$ dividing $a$, and $(a)_{p^{\prime}}=\frac{a}{(a)_{p}}$.

The degree of any ordinary character in this block is divisible by $\left(\frac{|G|}{\left|C_{G^{*}}(w)\right|}\right)_{p^{\prime}}$ for some $\ell$-regular element $w$ because of the following fact:

Proposition 2.3.3. 24, Proposition 1] Let $\rho$ be an irreducible $\ell$-Brauer character of $G$. Assume that $\rho$ lies in the union of $\ell$-blocks $\mathcal{E}_{\ell}(G, s)$ for some semisimple $\ell^{\prime}$-element $s$ of $G^{*}$. Then $\left(\frac{|G|}{\left|C_{G^{*}}(s)\right|}\right)_{p^{\prime}}$ divides $\rho(1)$.

Finally, in this section, we will mention that GAP, [17, was very useful in our computations, as it contains many ordinary and modular character tables. The following commands are used to call the ordinary and modular character tables of a group, for example, $A_{5}$ and $A_{5} \bmod 5$ : 
gap> Display (CharacterTable("A5")) ;

A5

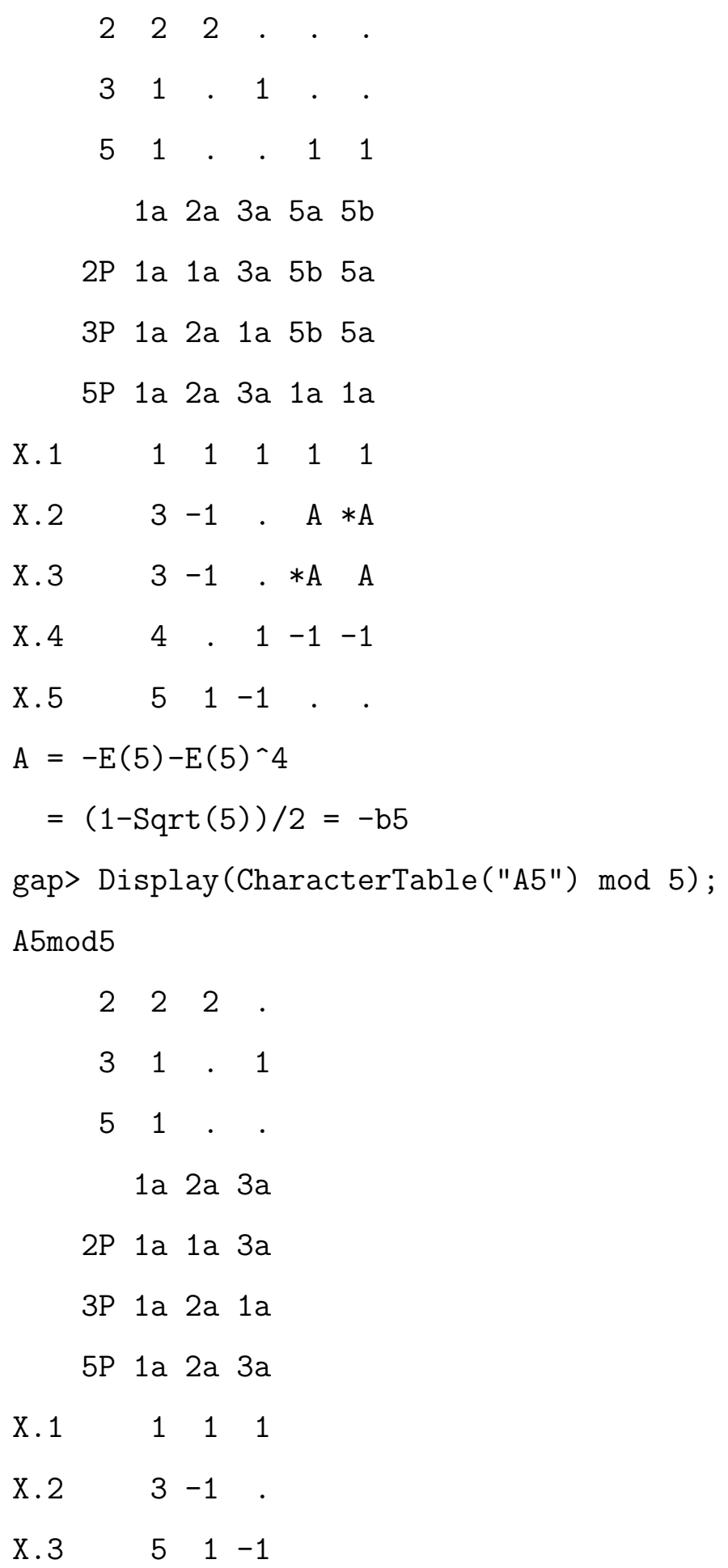


The character table displays the number of irreducible characters (the rows), as well as (the orders of) representatives of the conjugacy classes of the finite group (the columns). The table displays the irrationalities that the characters take on, allowing us to compute the fields of values of the irreducible characters.

\subsection{Structure of Maximal Tori}

In this section, we will discuss the (cyclic) structure of the maximal tori of the finite groups of Lie type. None of the work contained in this section is our original work, and we rely heavily on the work provided in [5], [50], and [27].

\subsubsection{The Classical Groups of Lie Type}

Theorem 2.4.1 (Theorem 2.1, [5]). Let $n \geq 2$ and $\left\{n_{1}, n_{2}, \ldots, n_{s}\right\}$ be a partition of $n$. Let $T$ be a maximal torus of $\mathrm{SL}_{n}(q)$ corresponding to an element of the Weyl group with cycle type $\left(n_{1}\right)\left(n_{2}\right) \cdots\left(n_{s}\right)$. For $1 \leq i \leq s$, define

$$
d_{i}=\operatorname{lcm}_{1 \leq j_{1}<\cdots<j_{i} \leq s} \operatorname{gcd}\left\{q^{n_{j_{1}}}-1, \ldots, q^{n_{j_{i}}}-1\right\}
$$

Then $d_{i}$ divides $d_{i^{\prime}}$ for $i>i^{\prime}$ and

$$
T \cong d_{1} \times d_{2} \times \cdots \times d_{s-1} \times \frac{d_{s}}{q-1} .
$$

Remark 2.4.2. Notice that this implies that $\mathrm{SL}_{n}(q)$ has cyclic maximal tori of orders $\frac{q^{n}-1}{q-1}$ and $q^{n-1}-1$, corresponding to an $n$-cycle and an $n-1$-cycle (respectively) in the Weyl group. Further, the maximal tori of $\mathrm{PGL}_{n}(q)$ are isomorphic to those of $\mathrm{SL}_{n}(q)$, [6, Proposition 4.4.1].

Theorem 2.4.3 (Theorem 2.2, [5]). Let $n \geq 2$ and $\left\{n_{1}, n_{2}, \ldots, n_{s}\right\}$ be a partition of $n$. Let $T$ be a maximal torus of $\mathrm{SU}_{n}(q)$ corresponding to an element of the Weyl group with cycle type $\left(n_{1}\right)\left(n_{2}\right) \cdots\left(n_{s}\right)$. For $1 \leq i \leq s$, define

$$
d_{i}=\operatorname{lcm}_{1 \leq j_{1}<\cdots<j_{i} \leq s} \operatorname{gcd}\left\{q^{n_{j_{1}}}-(-1)^{n_{j_{1}}}, \ldots, q^{n_{j_{i}}}-(-1)^{n_{j_{i}}}\right\}
$$


Then $d_{i}$ divides $d_{i^{\prime}}$ for $i>i^{\prime}$ and

$$
T \cong d_{1} \times d_{2} \times \cdots \times d_{s-1} \times \frac{d_{s}}{q+1}
$$

Remark 2.4.4. Notice that this implies that $\mathrm{SU}_{n}(q)$ has cyclic maximal tori of orders $\frac{q^{n}+1}{q+1}$ and $q^{n-1}-1$, corresponding to an $n$-cycle and an $n-1$-cycle (respectively) in the Weyl group when $n$ is odd, and $\mathrm{SU}_{n}(q)$ has cyclic maximal tori of orders $\frac{q^{n}-1}{q+1}$ and $q^{n-1}+1$, corresponding to an $n$-cycle and an $n-1$-cycle (respectively) in the Weyl group when $n$ is even. We remark further that the maximal tori of $\operatorname{PGU}_{n}(q)$ are isomorphic to the maximal tori of $\mathrm{SU}_{n}(q)$, [6, Proposition 4.4.1].

For the remaining classical groups, we will need the notion of positive and negative cycles in determining the structure of the Weyl groups corresponding to the maximal tori. Again, we follow [5] for our notation.

Dropping the signs from $\{1,2, \ldots, n,-1,-2, \ldots,-n\}$, we obtain an obvious homomorphism from $S l_{n}$, the group of permutations $\tau$ on $\{1,2, \ldots, n,-1,-2, \ldots,-n\}$ with $\tau(-i)=-\tau(i)$ into $S_{n}$, the symmetric group on $n$ letters. Assume that $\tau \in S l_{n}$ is mapped into a cycle $\left(i_{1} \cdots i_{k}\right)$ of $S_{n}$ and fixes all values distinct from $\pm i_{1}, \pm i_{2}, \ldots, \pm i_{k}$. If $\tau\left(i_{1}\right)=i_{2}$, then $\tau$ is a positive cycle of length $k$, and if $\tau\left(i_{1}\right)=-i_{2}$, then $\tau$ is a negative cycle of length $k$. As the image of $\tau$ in $S_{n}$ can be factored as a product of disjoint cycles, we may express $\tau$ as a product of disjoint positive and negative cycles.

Suppose $n=n^{\prime}+n^{\prime \prime}$, and let $\left\{n_{1}, \ldots, n_{k}\right\}$ and $\left\{n_{k+1}, \ldots, n_{s}\right\}$ be partitions of $n^{\prime}$ and $n^{\prime \prime}$, respectively. Then we denote the cycle type of $\left\{n_{1}, \ldots, n_{k},-n_{k+1}, \ldots,-n_{s}\right\}$ by $\left(n_{1}\right) \cdots\left(n_{k}\right)\left(\overline{n_{k+1}}\right) \cdots\left(\overline{n_{s}}\right)$. A standard element with this cycle type is a permutation $\tau^{\prime} \tau^{\prime \prime}$ where $\tau^{\prime}$ is the product of a standard element of cycle type $\left(n_{1}\right) \ldots\left(n_{k}\right)$ in $S_{n}$ and a permutation such as

$\left(-1, \ldots,-n_{1}\right)\left(-n_{1}-1, \ldots,-n_{1}-n_{2}\right) \ldots\left(-n_{1}-\cdots-n_{k-1}-1, \ldots,-n_{1}-\cdots-n_{k}\right)$,

and $\tau^{\prime \prime}$ is a product of negative cycles. 
Using these decompositions, we are now able to describe the cyclic structure of the maximal tori in the symplectic and the so-called spin groups.

Definition 2.4.5. Let $\mathcal{G}$ be a simple, simply connected algebraic group of type $B_{n}$ over $\overline{\mathbb{F}}_{p}$, and let $F$ be the standard $q$-power Frobenius morphism of $\mathcal{G}, q=p^{f}$. Then $\mathcal{G}^{F}$ is the spin group $G=\operatorname{Spin}_{2 n+1}(q)$. Now, let $\mathcal{G}^{*}$ be a simple, adjoint algebraic group of type $B_{n}$. Then $G^{*}:=\mathcal{G}^{* F}=\operatorname{PCSp}_{2 n}(q)$ is the projective conformal symplectic group. We have that $G$ and $G^{*}$ are in duality (see page $120,[6]$ ).

Let $\mathcal{G}$ be a simple, simply connected algebraic group of type $D_{n}$ over $\overline{\mathbb{F}}_{p}$, and let $F$ be the standard $q$-power Frobenius morphism of $\mathcal{G}, q=p^{f}$. Then $\mathcal{G}^{F}$ is the spin group $G=\operatorname{Spin}_{2 n}^{+}(q)$, corresponding to a quadratic form over $\mathbb{F}_{q}$ of maximal Witt defect. Now, let $\mathcal{G}^{*}$ be a simple, adjoint algebraic group of type $D_{n}$. Then $G^{*}:=$ $\mathcal{G}^{* F}=\mathrm{P}\left(\mathrm{CO}_{2 n}(q)^{\circ}\right)$, where $\mathrm{CO}_{2 n}(q)$ is the conformal orthogonal group corresponding to a quadratic form over $\mathbb{F}_{q}$ of maximal Witt defect, and $\mathrm{CO}_{2 n}(q)^{\circ}$ is the subgroup of index 2 that does not interchange the maximal isotropic subspaces of dimension $n$ of $V$ defined in Section 2.1.1. We have that $G$ and $G^{*}$ are in duality, [6].

Let $\mathcal{G}$ be a simple, simply connected algebraic group of type ${ }^{2} D_{n}$ over $\overline{\mathbb{F}}_{p}$, and $F$ is the standard $q$-power Frobenius morphism of $\mathcal{G}, q=p^{f}$. Then $\mathcal{G}^{F}$ is the spin group $G=\operatorname{Spin}_{2 n}^{-}(q)$, corresponding to a quadratic form over $\mathbb{F}_{q}$ of Witt defect 1 and of maximal Witt defect over $\mathbb{F}_{q^{2}}$. Now, let $\mathcal{G}^{*}$ be a simple, adjoint algebraic group of type ${ }^{2} D_{n}$. Then $G^{*}:=\mathcal{G}^{* F}=\mathrm{P}\left(\mathrm{CO}_{2 n}^{-}(q)^{\circ}\right)$, where $\mathrm{CO}_{2 n}^{-}(q)$ is the conformal orthogonal group corresponding to a quadratic form over $\mathbb{F}_{q}$ of Witt defect 1 and of maximal defect over $\mathbb{F}_{q^{2}}$, and $\mathrm{CO}_{2 n}(q)^{\circ}$ is the subgroup of index 2 that does not interchange the maximal isotropic subspaces of dimension $n$ (over $\mathbb{F}_{q^{2}}$ ) of $V$ defined in Section 2.1.1. We have that $G$ and $G^{*}$ are in duality, [6].

Theorem 2.4.6 (Theorem 3, [5]). Let $n \geq 2$ and $\left\{n_{1}, n_{2}, \ldots, n_{s}\right\}$ be a partition of $n$. Let $T$ be a maximal torus of $\operatorname{Sp}_{2 n}(q)$ corresponding to an element of the Weyl group with cycle type $\left(n_{1}\right)\left(n_{2}\right) \cdots\left(n_{k}\right)\left(\bar{n}_{k+1}\right) \cdots\left(\bar{n}_{s}\right)$. Let $\epsilon_{i}=+$ if $i \leq k$ and $\epsilon_{i}=-$ if $i>k$. 
Then

$$
T \cong\left(q^{n_{1}}-\epsilon_{1} 1\right) \times\left(q^{n_{2}}-\epsilon_{2} 1\right) \times \cdots \times\left(q^{n_{s}}-\epsilon_{s} 1\right) .
$$

Remark 2.4.7. Notice that this implies that $\mathrm{Sp}_{2 n}(q)$ has cyclic maximal tori of orders $q^{n}-1$ and $q^{n}+1$, corresponding to the positive and negative $n$-cycles, respectively. Further, the maximal tori of $\mathrm{SO}_{2 n+1}(q)$ are isomorphic to those of $\operatorname{Sp}_{2 n}(q)$, [6, Proposition 4.4.1].

Theorem 2.4.8 (Theorem 1, [50]). Let $n \geq 2$ and $\left\{n_{1}, n_{2}, \ldots, n_{s}\right\}$ be a partition of $n$. Let $T$ be a maximal torus of $\operatorname{Spin}_{2 n}^{ \pm}(q)$ or $\operatorname{Spin}_{2 n+1}(q)$ corresponding to an element of the Weyl group with cycle type $\left(n_{1}\right)\left(n_{2}\right) \cdots\left(n_{k}\right)\left(\bar{n}_{k+1}\right) \cdots\left(\bar{n}_{s}\right)$. Let $\epsilon_{i}=+$ if $i \leq k$ and $\epsilon_{i}=-$ if $i>k$.

1. If there are odd elements $n_{i} \in\left\{n_{1}, \ldots, n_{k}\right\}$ and $n_{j} \in\left\{n_{k+1}, \ldots, n_{s}\right\}$, then

$$
T \cong\left(q^{n_{i}}-1\right)\left(q^{n_{j}}+1\right) \times \prod_{m \neq i, j}\left(q^{n_{m}}-\epsilon_{m} 1\right)
$$

2. If there is an odd element $n_{i}$ in either $\left\{n_{1}, \ldots, n_{k}\right\}$ or $\left\{n_{k+1}, \ldots, n_{s}\right\}$ but not both and an even element $n_{j}$ in $\left\{n_{k}, \ldots, n_{s}\right\}$, then

$$
T \cong\left(q^{n_{i}}-\epsilon_{i} 1\right)\left(q^{n_{j}}+1\right) \times \prod_{m \neq i, j}\left(q^{n_{m}}-\epsilon_{m} 1\right)
$$

3. If all elements in $\left\{n_{1}, \ldots, n_{k}\right\}$ are even and $\left\{n_{k+1}, \ldots, n_{s}\right\}=\emptyset$, then choose $n_{i} \in\left\{n_{1}, \ldots, n_{k}\right\}$ with minimal 2-part. In this case,

$$
T \cong\left(q^{n_{i} / 2}-1\right) \times\left(q^{n_{i} / 2}+1\right) \times \prod_{m \neq i}\left(q^{n_{m}}-1\right) .
$$

4. In all other cases,

$$
T \cong \prod_{i}\left(q^{n_{i}}-\epsilon_{i} 1\right)
$$


Remark 2.4.9. This implies that $\operatorname{Spin}_{2 n+1}(q)$ has maximal tori of orders $q^{n}-1$ and $q^{n}+1$, corresponding to the positive and negative $n$-cycles, respectively. Further, $\operatorname{Spin}_{2 n}^{+}(q)$ has maximal tori of orders $q^{n}-1$ and $\left(q^{n-1}+1\right)(q+1)$, corresponding to the positive $n$-cycle and $(\overline{n-1}, \overline{1})$ cycle, respectively. Lastly, we notice that $\operatorname{Spin}_{2 n}^{-}(q)$ has maximal tori of orders $q^{n}+1$ and $\left(q^{n-1}+1\right)(q-1)$, corresponding to the negative $n$ cycle and $(1, \overline{n-1})$ cycle, respectively. As usual, the maximal tori of these groups are isomorphic to the tori of their dual groups, $\operatorname{PCSp}_{2 n}(q), \mathrm{PCO}_{2 n}(q)^{\circ}$, and $\mathrm{PCO}_{2 n}^{-}(q)^{\circ}$, respectively, [6, Proposition 4.4.1].

\subsubsection{The Exceptional Groups of Lie Type}

Using the results from [27], we see that the cyclic structure of the maximal tori of the exceptional groups of Lie type are as follows:

1. $\mathrm{E}_{6}(q)$ :

$$
\begin{gathered}
(q-1) \times(q-1) \times(q-1) \times(q-1) \times(q-1) \times(q-1) \\
(q-1) \times(q-1) \times(q-1) \times(q-1) \times\left(q^{2}-1\right) \\
(q-1) \times(q-1) \times\left(q^{2}-1\right) \times\left(q^{2}-1\right) \\
(q-1) \times(q-1) \times(q-1) \times\left(q^{3}-1\right) \\
\left(q^{2}-1\right) \times\left(q^{2}-1\right) \times\left(q^{2}-1\right) \\
(q-1) \times\left(q^{2}-1\right) \times\left(q^{3}-1\right) \\
(q-1) \times(q-1) \times\left(q^{4}-1\right) \\
(q+1) \times(q+1) \times\left(q^{2}-1\right) \times\left(q^{2}-1\right) \\
\left(q^{2}-1\right) \times(q+1)\left(q^{3}-1\right) \\
(q-1) \times\left(q^{2}+q+1\right) \times\left(q^{3}-1\right)
\end{gathered}
$$




$$
\begin{gathered}
\left(q^{2}-1\right) \times\left(q^{4}-1\right) \\
(q-1) \times\left(q^{5}-1\right) \\
\left(q^{2}-1\right) \times(q-1)\left(q^{3}+1\right) \\
(q-1)\left(q^{2}+1\right) \times(q-1)\left(q^{2}+1\right) \\
\left(q^{2}+q+1\right) \times(q+1) \times\left(q^{3}-1\right) \\
(q+1) \times(q+1) \times\left(q^{4}-1\right) \\
(q+1)\left(q^{5}-1\right) \\
\left(q^{2}+q+1\right) \times(q-1) \times\left(q^{3}+1\right) \\
\left(q^{2}-1\right)\left(q^{4}-1\right) \\
(q-1)\left(q^{2}+1\right)\left(q^{3}+1\right) \\
\left(q^{2}-q+1\right) \times\left(q^{4}+q^{2}+1\right) \\
q^{6}+q^{3}+1 \\
\left(q^{2}+q+1\right)\left(q^{4}-q^{2}+1\right) \\
(q+1) \times\left(q^{2}+q+1\right) \times\left(q^{2}+q+1\right)
\end{gathered}
$$

2. $\mathrm{E}_{7}(q)$ :

$(q-1) \times$ any element from the set of maximal tori of $\mathrm{E}_{6}(q)$

$$
\begin{gathered}
(q-1) \times(q+1) \times(q+1) \times\left(q^{2}-1\right) \times\left(q^{2}-1\right) \\
(q-1) \times\left(q^{3}-1\right) \times\left(q^{3}-1\right) \\
(q-1) \times\left(q^{2}-1\right) \times\left(q^{4}-1\right) \\
\left(q^{3}-1\right) \times(q+1)\left(q^{3}-1\right)
\end{gathered}
$$




$$
\begin{gathered}
(q-1) \times(q+1) \times(q+1) \times\left(q^{4}-1\right) \\
(q-1) \times(q+1)\left(q^{5}-1\right) \\
(q-1) \times\left(q^{6}-1\right) \\
(q-1) \times\left(q^{2}-1\right)\left(q^{4}+1\right) \\
\left(q^{2}+q+1\right) \times\left(q^{2}+q+1\right) \times\left(q^{3}-1\right) \\
\left(q^{3}+1\right) \times\left(q^{3}-1\right) \times(q+1) \\
\left(q^{3}-1\right)\left(q^{4}-q^{2}+1\right) \\
(q-1)\left(q^{6}+q^{3}+1\right) \\
\left(q^{2}-q+1\right) \times(q-1)\left(q^{4}+q^{2}+1\right) \\
\left(q^{3}-1\right) \times\left(q^{4}-1\right) \\
\left(q^{5}-1\right) \times\left(q^{2}+q+1\right) \\
\left(q^{4}+1\right) \times(q-1)\left(q^{2}+1\right) \\
(q-1)\left(q^{2}+1\right) \times\left(q^{2}-1\right) \times\left(q^{2}+1\right) \\
(q-1
\end{gathered}
$$

3. $\mathrm{E}_{8}(q)$ :

$(q-1) \times$ any element from the set of maximal tori of $\mathrm{E}_{7}(q)$

$$
\begin{gathered}
(q-1) \times\left(q^{3}-1\right) \times\left(q^{4}-1\right) \\
(q-1) \times\left(q^{5}-1\right)\left(q^{2}+q+1\right) \\
\left(q^{2}-1\right) \times\left(q^{2}+1\right)(q-1) \times\left(q^{2}+1\right)(q-1) \\
(q-1) \times\left(q^{7}-1\right) \\
(q-1)\left(q^{4}+1\right) \times(q-1)\left(q^{2}+1\right)
\end{gathered}
$$




$$
\begin{aligned}
& \left(q^{2}-1\right) \times\left(q^{2}-1\right) \times\left(q^{2}-1\right) \times\left(q^{2}-1\right) \\
& \left(q^{2}-1\right) \times\left(q^{2}-1\right) \times(q+1)\left(q^{3}-1\right) \\
& \left(q^{2}-1\right) \times\left(q^{2}-1\right) \times\left(q^{4}-1\right) \\
& (q+1)\left(q^{3}-1\right) \times(q+1)\left(q^{3}-1\right) \\
& (q+1)\left(q^{3}-1\right) \times\left(q^{4}-1\right) \\
& \left(q^{4}-1\right) \times\left(q^{4}-1\right) \\
& \left(q^{2}-1\right) \times\left(q^{2}-1\right) \times\left(q^{2}+1\right) \times\left(q^{2}+1\right) \\
& \left(q^{2}-1\right) \times(q+1)\left(q^{5}-1\right) \\
& \left(q^{2}-1\right) \times\left(q^{6}-1\right) \\
& (q-1)\left(q^{2}+1\right) \times\left(q^{2}+1\right)\left(q^{3}-1\right) \\
& \left(q^{2}-1\right) \times\left(q^{2}-1\right)\left(q^{4}+1\right) \\
& \left(q^{2}+q+1\right) \times\left(q^{2}+q+1\right) \times(q+1)\left(q^{3}-1\right) \\
& (q+1)\left(q^{2}+q+1\right)\left(q^{5}-1\right) \\
& (q+1)\left(q^{2}+1\right)\left(q^{5}-1\right) \\
& (q+1)\left(q^{7}-1\right) \\
& q^{8}-1 \\
& \left(q^{2}-1\right) \times\left(q^{2}+1\right) \times\left(q^{4}+1\right) \\
& \left(q^{2}+1\right) \times\left(q^{2}+1\right) \times\left(q^{4}-1\right) \\
& (q+1)\left(q^{3}-1\right)\left(q^{4}+1\right) \\
& \left(q^{2}+1\right)\left(q^{6}-1\right) \\
& \left(q^{2}-1\right)\left(q^{2}+q+1\right)\left(q^{4}-q^{2}+1\right) \\
& \left(q^{2}-1\right)\left(q^{6}+q^{3}+1\right)
\end{aligned}
$$




$$
\begin{gathered}
\left(q^{2}-q+1\right) \times\left(q^{2}-q+1\right) \times(q+1)\left(q^{3}-1\right) \\
\left(q^{2}-1\right)\left(q^{6}+1\right) \\
\left(q^{2}+q+1\right) \times\left(q^{2}+q+1\right) \times\left(q^{2}+q+1\right) \times\left(q^{2}+q+1\right) \\
\left(q^{4}+q^{3}+q^{2}+q+1\right) \times\left(q^{4}+q^{3}+q^{2}+q+1\right) \\
\left(q^{2}+q+1\right) \times\left(q^{6}+q^{3}+1\right) \\
\left(q^{2}+1\right) \times\left(q^{2}+1\right) \times\left(q^{2}+1\right) \times\left(q^{2}+1\right) \\
\left(q^{2}+1\right) \times\left(q^{6}+1\right) \\
\left(q^{4}+1\right) \times\left(q^{4}+1\right) \\
\left(q^{4}-q^{2}+1\right)\left(q^{2}+q+1\right) \times\left(q^{2}+q+1\right) \\
\left(q^{4}+q^{2}+1\right) \times\left(q^{2}+q+1\right) \times\left(q^{2}-q+1\right) \\
q^{8}+q^{7}-q^{5}-q^{4}-q^{3}+q+1 \\
q^{8}-q^{4}+1 \\
q^{8}-q^{6}+q^{4}-q^{2}+1 \\
\left.q^{2}+1\right) \times\left(q^{4}-q^{2}+1\right)
\end{gathered}
$$

There are also maximal tori with structure given by replacing $q$ with $-q$ in any element from this list.

4. $\mathrm{F}_{4}(q)$ :

$$
\begin{gathered}
(q-1) \times(q-1) \times(q-1) \times(q-1) \\
(q-1) \times(q-1) \times\left(q^{2}-1\right) \\
(q-1) \times(q+1) \times\left(q^{2}-1\right) \\
\left(q^{2}-1\right) \times\left(q^{2}-1\right)
\end{gathered}
$$




$$
\begin{gathered}
(q-1) \times\left(q^{3}-1\right) \\
(q-1) \times(q-1)\left(q^{2}+1\right) \\
(q+1) \times(q+1) \times\left(q^{2}-1\right) \\
(q-1) \times\left(q^{2}+1\right)(q+1) \\
\frac{q^{4}-1}{(2, q-1)} \times(2, q-1) \\
\left(q^{3}+1\right)(q-1) \\
\left(q^{3}-1\right)(q+1) \\
(q+1) \times(q+1) \times(q+1) \times(q+1) \\
\left(q^{2}+q+1\right) \times\left(q^{2}+q+1\right) \\
(q+1) \times\left(q^{2}+1\right)(q+1) \\
\left(q^{2}-q+1\right) \times\left(q^{2}-q+1\right) \\
q^{4}-q^{2}+1 \\
q^{4}+1 \\
\left(q^{2}+1\right) \times\left(q^{3}+1\right)
\end{gathered}
$$

5. $\mathrm{G}_{2}(q)$ :

$$
\begin{gathered}
(q-1) \times(q-1) \\
q^{2}-1 \\
(q+1) \times(q+1) \\
q^{2}-q+1 \\
q^{2}+q+1
\end{gathered}
$$


6. ${ }^{2} \mathrm{~B}_{2}(q), q=2^{2 m+1}$ for some $m \geq 1$ :

$$
\begin{gathered}
q-1 \\
q+\sqrt{2 q}+1 \\
q-\sqrt{2 q}+1
\end{gathered}
$$

7. ${ }^{3} \mathrm{D}_{4}(q)$ :

$$
\begin{gathered}
(q-1) \times\left(q^{3}-1\right) \\
(q+1) \times\left(q^{3}+1\right) \\
\left(q^{3}-1\right)(q+1) \\
\left(q^{3}+1\right) \times(q-1) \\
\left(q^{2}+q+1\right) \times\left(q^{2}+q+1\right) \\
\left(q^{2}-q+1\right) \times\left(q^{2}-q+1\right) \\
q^{4}-q^{2}+1
\end{gathered}
$$

8. ${ }^{2} \mathrm{E}_{6}(q)$ : The maximal tori of ${ }^{2} \mathrm{E}_{6}(q)$ are obtained by replacing $q$ with $-q$ in the list of maximal tori of $\mathrm{E}_{6}(q)$.

9. ${ }^{2} \mathrm{~F}_{4}(q), q=2^{2 m+1}$ for some $m \geq 1$ :

$$
\begin{gathered}
(q-1) \times(q-1) \\
q^{2}-1 \\
(q-1) \times(q-\sqrt{2 q}+1) \\
(q-1) \times(q+\sqrt{2 q}+1)
\end{gathered}
$$




$$
\begin{gathered}
q^{2}+1 \\
(q-\sqrt{2 q}+1) \times(q-\sqrt{2 q}+1) \\
(q+\sqrt{2 q}+1) \times(q+\sqrt{2 q}+1) \\
(q+1) \times(q+1) \\
q^{2}-q+1 \\
q^{2}-\sqrt{2 q^{3}}+q-\sqrt{2 q}+1 \\
q^{2}+\sqrt{2 q^{3}}+q+\sqrt{2 q}+1
\end{gathered}
$$

10. ${ }^{2} \mathrm{G}_{2}(q), q=3^{2 m+1}$ for some $m \geq 1$ :

$$
\begin{gathered}
q-1 \\
\frac{(q+1)}{2} \times 2 \\
q+\sqrt{3 q}+1 \\
q-\sqrt{3 q}+1
\end{gathered}
$$




\section{ZSIGMONDY PRIMES}

In this chapter, we will define and discuss Zsigmondy primes, which we will see are essential in proving our main thesis results. Some results in this section are joint work with Andrew MacLaughlin (another graduate student at the University of Arizona), and I will cite his name wherever credit is due. First, we will define Zsigmondy primes (also known as primitive prime divisors) and large Zsigmondy primes. Then we will state known facts, and prove our own results, including an application to the special linear group.

\subsection{Definitions and Known Facts}

Definition 3.1.1. Let $q$ and $n$ be integers greater than 1. A Zsigmondy prime or primitive prime divisor for $(q, n)$ is a prime $p$ such that $p \mid q^{n}-1$ but $p \nmid q^{i}-1$ for $1 \leq i \leq n-1$. We define $|n|_{p}$ as the $p$ part of $n$, meaning $|n|_{p}$ satisfies $|n|_{p}=p^{k}$ where $p^{k} \mid n$ but $p^{k+1} \nmid n$. A large Zsigmondy prime for $(q, n)$ is a Zsigmondy prime $p$ for $(q, n)$ which also satisfies $\left|q^{n}-1\right|_{p}>n+1$.

An obvious question that arises with this definition concerns the existence of such prime numbers. Karl Zsigmondy and Walter Feit have proven several theorems that state the exact conditions on $q$ and $n$ to ensure the existence of primitive prime divisors and large Zsigmondy primes:

Theorem 3.1.2 (Zsigmondy's Theorem, 41]). Let $q$ and $n$ be integers greater than 1. There exists a prime divisor $p$ of $q^{n}-1$ such that $p$ does not divide $q^{j}-1$ for all $j, 0<j<n$, except in the following cases:

(i) $n=2$ and $q=2^{s}-1$ where $s \geq 2$.

(ii) $n=6$ and $q=2$. 
That is, for any $n>2, q>1,(q, n) \neq(2,6)$, there exists a Zsigmondy prime for $(q, n)$. Walter Feit proves the following theorem in [13].

Theorem 3.1.3 (Theorem B, [13]). Let $N>0$ be a positive integer. Then for all but a finite number of pairs of integers $(q, n)$ with $q>1$ and $n>2$, there exists a Zsigmondy prime $p$ with $\left|q^{n}-1\right|_{p}>n N+1$.

In particular, taking $N=1$, we see that for all but finitely many pairs $(q, n)$ with $n>2$ and $q>1$, there is a large Zsigmondy prime for $(q, n)$. In fact, [13] provides a complete list of exceptions for the case $N=1$, including the case $n=2$ :

Theorem 3.1.4 (Theorem A, [13]). If $q$ and $n$ are integers greater than 1, then there exists a large Zsigmondy prime for $(q, n)$ except in the following cases:

(i) $n=2$ and $q=2^{s} 3^{t}-1$ for some $s \in \mathbb{N}$ and $t=0$ or 1 .

(ii) $q=2$ and $n=4,6,10,12$ or 18

(iii) $q=3$ and $n=4$ or 6

(iv) $(q, n)=(5,6)$.

Let $\phi(n)=\#\{1 \leq m \leq n \mid(m, n)=1\}$ represent Euler's totient function, and let $\Phi_{n}(x)=\prod_{i=1}^{\phi(n)}\left(x-\xi_{i}\right)$ denote the $n^{\text {th }}$ cyclotomic polynomial, where $\xi_{i}$ are the primitive $n^{\text {th }}$ roots of unity. Observe that $\Phi_{n}(q)=\left|\Phi_{n}(q)\right|>0$ for all integers $q \geq 2$. The following lemmas were critical in the proofs of the above theorems, and were essential in proving our own results.

Lemma 3.1.5 ([41], Lemma 1). Let $q>1$ and $n=p^{i} r$ be integers, where $p$ is a prime, $i \geq 1$ and $r$ is a positive integer not divisible by $p$. Let $b=q^{p^{i-1}}$. Then

$$
\Phi_{n}(q)>\left(b^{p-2}(b-1)\right)^{\phi(r)} .
$$

Proof. From Corollary 4 in [18], we know that

$$
\Phi_{n}(q)=\frac{\Phi_{r}\left(q^{p^{i}}\right)}{\Phi_{r}\left(q^{p^{i-1}}\right)}=\frac{\Phi_{r}\left(b^{p}\right)}{\Phi_{r}(b)}
$$


Now, we see that for primitive $r$ th roots of unity $\xi_{i}$,

$$
\frac{\Phi_{r}\left(b^{p}\right)}{\Phi_{r}(b)}=\frac{\prod_{i=1}^{\phi(r)}\left(b^{p}-\xi_{i}\right)}{\prod_{i=1}^{\phi(r)}\left(b-\xi_{i}\right)}>\left(\frac{b^{p}-1}{b+1}\right)^{\phi(r)}
$$

Notice that $b^{p}-1 \geq b^{p}-b^{p-2}=b^{p-2}\left(b^{2}-1\right)$ so

$$
\left(\frac{b^{p}-1}{b+1}\right)^{\phi(r)} \geq\left(\frac{b^{p-2}\left(b^{2}-1\right)}{b+1}\right)^{\phi(r)}=\left(b^{p-2}(b-1)\right)^{\phi(r)} .
$$

That is, $\Phi_{n}(q)>\left(b^{p-2}(b-1)\right)^{\phi(r)}$.

Lemma 3.1.6 ([13], Lemma 2.2). Let $q, n$ be integers with $q>1$ and $n>2$. Let $N$ be a positive integer. Suppose that if $p$ is a Zsigmondy prime for $(q, n)$ then $\left|q^{n}-1\right|_{p} \leq n N+1$. Then

$$
\Phi_{n}(q) \leq n(N n+1)^{N}
$$

Proof. Observe that if $p$ is any Zsigmondy prime for $(q, n)$, it must be the case that $p \equiv 1 \bmod n$. Hence there are at most $N$ Zsigmondy primes $p$ for $(q, n)$, each satisfying $p \leq n N+1$. Let $S$ be the set of Zsigmondy primes for $(q, n)$. Define $Z=\prod_{p \in S}\left|\Phi_{n}(q)\right|_{p}$. Since $\Phi_{n}(q) \mid q^{n}-1$, we have $\left|\Phi_{n}(q)\right|_{p} \leq\left|q^{n}-1\right|_{p}$ for all $p \in S$, and thus

$$
Z \leq\left(\left|q^{n}-1\right|_{p}\right)^{N} \leq(n N+1)^{N} .
$$

As shown in [1], $\Phi_{n}(q) \mid Z P(n)$, where $P(n)$ is the largest prime dividing $n$. Hence $\Phi_{n}(q) \leq Z P(n) \leq(n N+1)^{N} P(n)$. Of course $P(n) \leq n$, and the result follows.

Lemma 3.1.7. Let $n \geq 2$. Then $\phi(n) \geq \sqrt{\frac{n}{2}}$. 
Proof. Let $p$ be an odd prime, and let $m \geq 1$. Then $\phi\left(p^{m}\right)=(p-1) p^{m-1}$. Now, we see that

$$
(p-1) p^{m-1}-\sqrt{p^{m}}=p^{m}-p^{m-1}-p^{m / 2}=p^{m}\left(1-\frac{1}{p}-\frac{1}{p^{m / 2}}\right)>0
$$

giving us

$$
\phi\left(p^{m}\right)=(p-1) p^{m-1}>\sqrt{p^{m}} .
$$

For coprime integers $a, b$, it is known that $\phi(a b)=\phi(a) \phi(b)$. This implies that if $q>1$ is an odd integer, then $\phi_{n}(q) \geq \sqrt{q}$.

For an integer $m \geq 1$, we see that

$$
\phi\left(2^{m}\right)=2^{m-1} \geq 2^{(m-1) / 2}=\sqrt{2^{m} / 2}
$$

Finally, for any integer $n$ with $n>1$, we may write $n=2^{m} q$ with $m$ a non-negative integer and $q$ and odd integer. Noting that $\phi(1)=1$, observe

$$
\phi(n)=\phi\left(2^{m} q\right)=\phi\left(2^{m}\right) \phi(q) \geq \sqrt{\frac{2^{m}}{2}} \sqrt{q}=\sqrt{\frac{n}{2}} .
$$

Lemma 3.1.8. Let $q, n, N$ be integers with $q>1, n>2$, and $N \geq 1$. If no Zsigmondy prime $p$ exists for $(q, n)$ with $\left|q^{n}-1\right|_{p}>n N+1$, then

$$
q^{\sqrt{n / 8}}<n(n N+1)^{N}
$$

Proof. This follows immediately from the three previous lemmas.

\subsection{The Case $N=2$}

In this section, we will restrict our attention to the case $N=2$. First, we consider the case when $n=2$ as there are no Zsigmondy primes for $(q, 2)$ with $q=2^{s}-1$, $s \geq 1$ : 
Proposition 3.2.1 (MacLaughlin, T.). Let $q>2$ be an integer. There exists a Zsigmondy prime $p$ satisfying $\left|q^{2}-1\right|_{p}>5$ unless $q=2^{m} 3^{r} 5^{s}-1$, where $m$ is a non-negative integer and $r, s \in\{0,1\}$.

Proof. By definition, $p$ is a Zsigmondy prime if $p \mid q^{2}-1$ and $p \nmid q-1$. It immediately follows that for $p$ to be a Zsigmondy prime, $p \neq 2$ and $p \mid q+1$. If $p>5$, then clearly $\left|q^{2}-1\right|_{p}>5$. As a result, for $q$ to be an exception, $q+1$ must have no prime divisors greater than 5 . Note that $3^{2}$ and $5^{2}$ are both greater than 5 . We may conclude that $q$ is an exception only when $q+1$ has prime factorization $2^{m} 3^{r} 5^{s}$ where $m \in \mathbb{Z}_{\geq 0}$ and $r, s \in\{0,1\}$. This is equivalent to stating $q=2^{m} 3^{r} 5^{s}-1$, where $m$ is a non-negative integer and $r, s \in\{0,1\}$.

Now, we provide the full results for $N=2$ :

Theorem 3.2.2 (MacLaughlin, T.). Let $q$ and $n$ be integers greater than 1 . Then there exists a Zsigmondy prime $p$ for $(q, n)$ with $\left|q^{n}-1\right|_{p}>2 n+1$ except in the following cases:

(i) $n=2$ and $q=2^{m} 3^{r} 5^{s}-1$, where $m$ is a non-negative integer and $r, s \in\{0,1\}$.

(ii) $q=2$ and $n=3,4,6,8,10,12,18$, or 20 .

(iii) $q=3$ and $n=4,6$, or 18 .

(iv) $q=4$ and $n=3$ or 6 .

(v) $(q, n)=(5,6),(10,6)$, or $(17,6)$.

Proof. We first used Lemma 3.1 .8 to establish rough bounds for both $q$ and $n$. Of course, the relation in Lemma 3.1 .8 implies that

$$
q<\left[n(2 n+1)^{2}\right]^{\frac{4}{\sqrt{n}}}
$$

We notice that $f(n)=\left[n(2 n+1)^{2}\right]^{\frac{4}{\sqrt{n}}}$ is decreasing for $n>4$ and that $2>[n(2 n+$ $\left.1)^{2}\right]^{\frac{4}{\sqrt{n}}}$ for $n=35,950$. Since $q>1$ and is an integer, an upper bound for $n$ is 35,949 . 
We may use this same relation over $3 \leq n \leq 35,949$ to obtain an upper bound on $q$ of 104,976 . The results are summarized in the following table:

\begin{tabular}{cc|cc|cc|cc}
\hline \hline$n$ & $\max q$ & $n$ & $\max q$ & $n$ & $\max q$ & $n$ & $\max q$ \\
\hline 3 & 101205 & 86 & 582 & 169 & 174 & 276 & 80 \\
4 & 104976 & 87 & 569 & 170 & 173 & 279 & 79 \\
5 & 94657 & 88 & 557 & 171 & 171 & 281 & 78 \\
6 & 81066 & 89 & 545 & 172 & 169 & 284 & 77 \\
7 & 68207 & 90 & 534 & 173 & 168 & 286 & 76 \\
8 & 57200 & 91 & 523 & 174 & 166 & 289 & 75 \\
9 & 48120 & 92 & 512 & 175 & 165 & 291 & 74 \\
10 & 40727 & 93 & 502 & 176 & 163 & 294 & 73 \\
11 & 34719 & 94 & 492 & 177 & 161 & 297 & 72 \\
12 & 29821 & 95 & 482 & 178 & 160 & 300 & 71 \\
13 & 25805 & 96 & 473 & 179 & 158 & 303 & 70 \\
14 & 22490 & 97 & 464 & 180 & 157 & 306 & 69 \\
15 & 19733 & 98 & 455 & 181 & 156 & 309 & 68 \\
16 & 17424 & 99 & 447 & 182 & 154 & 312 & 67 \\
17 & 15475 & 100 & 439 & 183 & 153 & 315 & 66 \\
18 & 13820 & 101 & 431 & 184 & 151 & 318 & 65 \\
19 & 12404 & 102 & 423 & 185 & 150 & 322 & 64 \\
20 & 11186 & 103 & 415 & 186 & 149 & 325 & 63 \\
21 & 10133 & 104 & 408 & 187 & 148 & 329 & 62 \\
22 & 9216 & 105 & 401 & 188 & 146 & 333 & 61 \\
\hline
\end{tabular}




\begin{tabular}{|c|c|c|c|c|c|c|c|}
\hline$n$ & $\max q$ & $n$ & $\max q$ & $n$ & $\max q$ & $n$ & $\max q$ \\
\hline 23 & 8414 & 106 & 394 & 189 & 145 & 337 & 60 \\
\hline 24 & 7709 & 107 & 387 & 190 & 144 & 341 & 59 \\
\hline 25 & 7087 & 108 & 381 & 191 & 143 & 345 & 58 \\
\hline 26 & 6535 & 109 & 374 & 192 & 141 & 349 & 57 \\
\hline 27 & 6044 & 110 & 368 & 193 & 140 & 353 & 56 \\
\hline 28 & 5605 & 111 & 362 & 194 & 139 & 358 & 55 \\
\hline 29 & 5211 & 112 & 356 & 195 & 138 & 363 & 54 \\
\hline 30 & 4857 & 113 & 351 & 196 & 137 & 368 & 53 \\
\hline 31 & 4537 & 114 & 345 & 197 & 136 & 373 & 52 \\
\hline 32 & 4247 & 115 & 340 & 198 & 135 & 378 & 51 \\
\hline 33 & 3984 & 116 & 335 & 199 & 133 & 383 & 50 \\
\hline 34 & 3745 & 117 & 329 & 200 & 132 & 389 & 49 \\
\hline 35 & 3526 & 118 & 324 & 201 & 131 & 395 & 48 \\
\hline 36 & 3326 & 119 & 320 & 202 & 130 & 401 & 47 \\
\hline 37 & 3142 & 120 & 315 & 203 & 129 & 407 & 46 \\
\hline 38 & 2973 & 121 & 310 & 204 & 128 & 414 & 45 \\
\hline 39 & 2818 & 122 & 306 & 205 & 127 & 421 & 44 \\
\hline 40 & 2674 & 123 & 301 & 206 & 126 & 428 & 43 \\
\hline 41 & 2541 & 124 & 297 & 207 & 125 & 436 & 42 \\
\hline 42 & 2418 & 125 & 293 & 208 & 124 & 444 & 41 \\
\hline 43 & 2304 & 126 & 289 & 209 & 123 & 452 & 40 \\
\hline 44 & 2198 & 127 & 285 & 210 & 122 & 461 & 39 \\
\hline 45 & 2099 & 128 & 281 & 212 & 121 & 470 & 38 \\
\hline 46 & 2006 & 129 & 277 & 213 & 120 & 480 & 37 \\
\hline 47 & 1920 & 130 & 273 & 214 & 119 & 490 & 36 \\
\hline 48 & 1839 & 131 & 270 & 215 & 118 & 500 & 35 \\
\hline 49 & 1764 & 132 & 266 & 216 & 117 & 512 & 34 \\
\hline 50 & 1693 & 133 & 262 & 217 & 116 & 524 & 33 \\
\hline 51 & 1626 & 134 & 259 & 218 & 115 & 537 & 32 \\
\hline 52 & 1563 & 135 & 256 & 220 & 114 & 550 & 31 \\
\hline 53 & 1504 & 136 & 252 & 221 & 113 & 565 & 30 \\
\hline 54 & 1448 & 137 & 249 & 222 & 112 & 580 & 29 \\
\hline 55 & 1396 & 138 & 246 & 223 & 111 & 597 & 28 \\
\hline 56 & 1346 & 139 & 243 & 225 & 110 & 615 & 27 \\
\hline 57 & 1299 & 140 & 240 & 226 & 109 & 634 & 26 \\
\hline 58 & 1255 & 141 & 237 & 227 & 108 & 655 & 25 \\
\hline
\end{tabular}




\begin{tabular}{|c|c|c|c|c|c|c|c|}
\hline$n$ & $\max q$ & $n$ & $\max q$ & $n$ & $\max q$ & $n$ & $\max q$ \\
\hline 59 & 1213 & 142 & 234 & 229 & 107 & 678 & 24 \\
\hline 60 & 1173 & 143 & 231 & 230 & 106 & 702 & 23 \\
\hline 61 & 1135 & 144 & 229 & 231 & 105 & 729 & 22 \\
\hline 62 & 1099 & 145 & 226 & 233 & 104 & 759 & 21 \\
\hline 63 & 1064 & 146 & 223 & 234 & 103 & 792 & 20 \\
\hline 64 & 1031 & 147 & 221 & 236 & 102 & 828 & 19 \\
\hline 65 & 1000 & 148 & 218 & 237 & 101 & 868 & 18 \\
\hline 66 & 971 & 149 & 216 & 239 & 100 & 914 & 17 \\
\hline 67 & 942 & 150 & 213 & 240 & 99 & 966 & 16 \\
\hline 68 & 915 & 151 & 211 & 242 & 98 & 1025 & 15 \\
\hline 69 & 890 & 152 & 208 & 244 & 97 & 1093 & 14 \\
\hline 70 & 865 & 153 & 206 & 245 & 96 & $1172 \leq n<1267$ & 13 \\
\hline 71 & 841 & 154 & 204 & 247 & 95 & $1267 \leq n<1380$ & 12 \\
\hline 72 & 819 & 155 & 202 & 249 & 94 & $1380 \leq n<1520$ & 11 \\
\hline 73 & 797 & 156 & 199 & 250 & 93 & $1520 \leq n<1694$ & 10 \\
\hline 74 & 776 & 157 & 197 & 252 & 92 & $1694 \leq n<1920$ & 9 \\
\hline 75 & 756 & 158 & 195 & 254 & 91 & $1920 \leq n<2223$ & 8 \\
\hline 76 & 737 & 159 & 193 & 256 & 90 & $2223 \leq n<2648$ & 7 \\
\hline 77 & 719 & 160 & 191 & 258 & 89 & $2648 \leq n<3287$ & 6 \\
\hline 78 & 701 & 161 & 189 & 260 & 88 & $3287 \leq n<4343$ & 5 \\
\hline 79 & 684 & 162 & 187 & 262 & 87 & $4343 \leq n<6373$ & 4 \\
\hline 80 & 668 & 163 & 185 & 264 & 86 & $6373 \leq n<11484$ & 3 \\
\hline 81 & 652 & 164 & 183 & 266 & 85 & $11484 \leq n \leq 35949$ & 2 \\
\hline 82 & 637 & 165 & 181 & 268 & 84 & & \\
\hline 83 & 622 & 166 & 180 & 270 & 83 & & \\
\hline 84 & 608 & 167 & 178 & 272 & 82 & & \\
\hline 85 & 595 & 168 & 176 & 274 & 81 & & \\
\hline
\end{tabular}

However, these bounds are impractical in terms of directly checking for exceptions. Lemma 3.1.7 yields the relation

$$
q<\left[n(2 n+1)^{2}\right]^{\frac{2}{\phi(n)}}
$$

Using GAP, we found the values of $\phi(n)$ for $3 \leq n \leq 35,949$. We were then able to directly compute new bounds for $q$ given $3 \leq n \leq 35,949$. We discovered $2>$ $\left[n(2 n+1)^{2}\right]^{\frac{2}{\phi(n)}}$ for $n>210$, narrowing our bound on $n$ from 35,950 to 210 . We also found the maximum value of $q$ to be bounded by 1,014. The results are summarized in the following table: 


\begin{tabular}{cc|cc|cc|cc}
\hline \hline$n$ & maximum $q$ & $n$ & maximum $q$ & $n$ & maximum $q$ & $n$ & maximum $q$ \\
\hline 3 & 147 & 24 & 15 & 50 & 3 & 82 & 2 \\
4 & 324 & 25 & 3 & 51 & 2 & 84 & 3 \\
5 & 24 & 26 & 6 & 52 & 3 & 86 & 2 \\
6 & 1014 & 27 & 3 & 54 & 4 & 88 & 2 \\
7 & 11 & 28 & 6 & 56 & 3 & 90 & 3 \\
8 & 48 & 29 & 2 & 57 & 2 & 96 & 2 \\
9 & 14 & 30 & 18 & 58 & 2 & 98 & 2 \\
10 & 66 & 31 & 2 & 60 & 5 & 100 & 2 \\
11 & 5 & 32 & 4 & 62 & 2 & 102 & 2 \\
12 & 86 & 33 & 3 & 63 & 2 & 108 & 2 \\
13 & 4 & 34 & 4 & 64 & 2 & 110 & 2 \\
14 & 22 & 35 & 2 & 66 & 4 & 114 & 2 \\
15 & 10 & 36 & 7 & 68 & 2 & 120 & 2 \\
16 & 11 & 38 & 3 & 70 & 3 & 126 & 2 \\
17 & 3 & 39 & 2 & 72 & 3 & 132 & 2 \\
18 & 29 & 40 & 4 & 74 & 2 & 138 & 2 \\
19 & 3 & 42 & 8 & 75 & 2 & 150 & 2 \\
20 & 13 & 44 & 3 & 76 & 2 & 168 & 2 \\
21 & 5 & 45 & 2 & 78 & 3 & 180 & 2 \\
22 & 8 & 46 & 3 & 80 & 2 & 210 & 2 \\
23 & 2 & 48 & 5 & & & & \\
\hline
\end{tabular}

The remaining cases may be further narrowed by solving the relation $\Phi_{n}(q) \leq$ $n(2 n+1)^{2}$, as stated in Lemma 3.1.6. The remaining list of possible exceptions is summarized in the following table:

\begin{tabular}{cc|cc|cc|cc}
\hline \hline$n$ & maximum $q$ & $n$ & maximum $q$ & $n$ & maximum $q$ & $n$ & maximum $q$ \\
\hline 3 & 11 & 8 & 6 & 13 & 2 & 40 & 2 \\
4 & 17 & 9 & 3 & 32 & 2 & 42 & 2 \\
5 & 4 & 10 & 8 & 34 & 2 & 48 & 2 \\
6 & 32 & 11 & 2 & 36 & 2 & 54 & 2 \\
7 & 3 & 12 & 9 & 38 & 2 & 60 & 2 \\
\hline
\end{tabular}

Checking this very small list directly for exceptions, we obtain the desired results. 


\subsection{Application to the Special Linear Group}

Before we prove the main theorem of this section, we require some machinery. For a group $G$, an element $g \in G$ is quadratic rational if $g$ is conjugate to $g^{k^{2}}$ for all integers $k$ such that $|g|$ and $k$ are coprime. We say that a group is quadratic rational if all of its elements are quadratic rational. We find the following lemma useful.

Lemma 3.3.1. Let $G$ be a finite group such that there exists an element $g \in G$ with $|g|=\ell^{b}$ for an odd prime $\ell$ and $b \in \mathbb{N}$. Then $g$ is quadratic rational if and only if $\chi(g)$ is quadratic rational for all irreducible characters $\chi$ of $G$. Furthermore, the generators of $\langle g\rangle$ fall into at most two conjugacy classes in $G$.

Proof. Let $\zeta$ be a primitive $\ell^{b}$ root of unity. Let $\Gamma=\operatorname{Gal}(\mathbb{Q}(\zeta) / \mathbb{Q}) \cong\left(\mathbb{Z} / \ell^{b} \mathbb{Z}\right)^{\times}$. Since $\zeta$ has odd prime power order, this is a cyclic group. Now, for some $k$ relatively prime to $\ell^{b}$, we have that $\sigma: \zeta \mapsto \zeta^{k}$ is a generator for $\Gamma$.

Let $\chi \in \operatorname{Irr}(G)$ be afforded by a representation $\Phi: G \rightarrow \mathrm{GL}_{d}(\mathbb{C})$. For any $h \in G$ with order $m$, it is known that $\Phi(h)$ is similar to a diagonal matrix whose entries are $m^{\text {th }}$ roots of unity. This implies that $\chi(g) \in \mathbb{Q}(\zeta)$. Also, observe

$$
\sigma(\chi(g))=\sigma\left(\sum_{i=1}^{d} \zeta_{i}\right)=\sigma\left(\sum_{i-1}^{d} \zeta^{n_{i}}\right)=\sum_{i=1}^{d} \sigma\left(\zeta^{n_{i}}\right)=\sum_{i=1}^{d} \zeta^{k n_{i}}=\sum_{i=1}^{d} \zeta_{i}^{k}=\chi\left(g^{k}\right)
$$

$(\Rightarrow)$ Suppose that $g \in G$ is quadratic rational. Then $g \sim g^{k^{2}}$. Observe $\sigma^{2}(\chi(g))=$ $\chi\left(g^{k^{2}}\right)=\chi(g)$. Since $\ell$ is an odd prime, $2 \mid \varphi\left(\ell^{b}\right)$. This implies that $\sigma^{2}$ is not a generator of $\Gamma$. It immediately follows that $\left[\Gamma:\left\langle\sigma^{2}\right\rangle\right]=2$. From the Fundamental Theorem of Galois Theory, the fixed field of $\sigma^{2}$ contained in $\mathbb{Q}(\zeta)$ is a degree 2 extension of $\mathbb{Q}$. As $\chi(g)$ is contained in the fixed field of $\sigma^{2}$, it follows that $\chi(g)$ is quadratic rational.

$(\Leftarrow)$ Now suppose that $\chi(g)$ is quadratic rational. This implies that $\mathbb{Q}(\chi(g))$ is a degree two extension of $\mathbb{Q}$. From the Fundamental Theorem of Galois Theory, $\mathbb{Q}(\chi(g))$ is the fixed field for an index 2 subgroup of $\Gamma$. Since $\Gamma$ is cyclic, it contains 
only one subgroup of index 2. As we have already stated, $\left[\Gamma:\left\langle\sigma^{2}\right\rangle\right]=2$, meaning $\left\langle\sigma^{2}\right\rangle$ fixes $\mathbb{Q}(\chi(g))$ and hence $\sigma^{2}$ fixes $\chi(g)$.

The Second Orthogonality Theorem states

$$
\sum_{\chi \in \operatorname{Irr}(G)} \chi(g) \overline{\chi(h)}=0
$$

if and only if $g$ and $h$ are not conjugate. Observe

$$
\sum_{\chi \in \operatorname{Irr}(G)} \chi\left(g^{k^{2}}\right) \overline{\chi(g)}=\sum_{\chi \in \operatorname{Irr}(G)} \sigma^{2}(\chi(g)) \overline{\chi(g)}=\sum_{\chi \in \operatorname{Irr}(G)} \chi(g) \overline{\chi(g)} \neq 0 .
$$

This implies that $g$ and $g^{k^{2}}$ are conjugate.

As $\sigma(\chi(g))=\chi\left(g^{k}\right)$, we may naturally identify $\sigma$ with a map $\psi: g \mapsto g^{k}$. We may write all of the primitive $\ell^{b}$ roots of unity as $\zeta^{m k}$ for some $m \in \mathbb{N}$. Similarly, we may write the generators of $\langle g\rangle$ as $g^{m k}$. It follows that successively applying $\sigma$ to $\zeta$ yields all of the primitive $\ell^{b}$ roots of unity. Since $\left[\Gamma:\left\langle\sigma^{2}\right\rangle\right]=2$, successively applying $\sigma^{2}$ to $\zeta$ yields half of the $\ell^{b}$ roots of unity. Similarly, successively applying $\psi^{2}$ to $g$ yields half of the generators of $\langle g\rangle$. The assumption that $g \sim g^{k^{2}}$ easily generalizes so that $g \sim \psi^{2 m}(g)$ for all $m \in \mathbb{N}$, implying $g$ is conjugate to at least half of the generators of $\langle g\rangle$. This argument may be applied to any generator of $\langle g\rangle$, meaning each generator of $\langle g\rangle$ is conjugate to half of the generators of $\langle g\rangle$. We conclude that the generators of $\langle g\rangle$ fall into at most two conjugacy classes.

We are now able to prove our main results concerning the special linear group.

Theorem 3.3.2 (MacLaughlin, T.). Let $q$ and $n$ be integers with $q>1, n>2$. For all but possibly sixteen pairs $(q, n), \mathrm{SL}_{n}(q)$ is not quadratic rational.

Proof. Assume $\mathrm{SL}_{n}(q)$ is quadratic rational. View $\mathbb{F}_{q^{n}}$ as an $n$-dimensional vector space $V$ over $\mathbb{F}_{q}$. We may view elements of $\mathbb{F}_{q^{n}}$ as elements of $\mathrm{GL}_{n}(q)$ via multiplication on $V$. As $\mathbb{F}_{q^{n}}^{*}$ is cyclic of order $q^{n}-1$, there exists an element $h \in \mathbb{F}_{q^{n}}^{*}$ of order $\frac{q^{n}-1}{q-1}$. Now, $h=t^{q-1}$ for some generator $t$ of $\mathbb{F}_{q^{n}}^{*}$. Hence $\operatorname{det}(h)=(\operatorname{det}(t))^{q-1}=1$. 
From Theorem 3.2.2, for all but sixteen pairs $(q, n)$, there exists a Zsigmondy prime $\ell$ with $\left|q^{n}-1\right|_{\ell}>2 n+1$. Note that $\ell \equiv 1 \bmod n$ so the previous statement implies $\left|q^{n}-1\right|_{\ell} \geq 3 n+1$. Also, note that $\ell \mid q^{n}-1$ but $\ell \nmid q-1$. This implies $\ell$ divides $\frac{q^{n}-1}{q-1}$.

As $\langle h\rangle$ has order $\frac{q^{n}-1}{q-1}$, there exists a $g \in\langle h\rangle$ such that $|g|=\left|q^{n}-1\right|_{\ell}=\ell^{b}$. Note that if $\ell=2$, then $\ell \mid\left(q^{n}-1\right)$ implies $2 \mid q-1$, which contradicts that $\ell$ is a Zsigmondy prime. This implies that $\ell$ is odd. From the previous lemma, the generators of $\langle g\rangle$ fall into at most two conjugacy classes in $\mathrm{SL}_{n}(q)$.

Before we continue, observe

$$
\begin{aligned}
\ell^{b} & \geq 3 n+1 \\
\ell^{b-1} & \geq \frac{3 n+1}{\ell} \\
\ell^{b-1}(\ell-1) & \geq(3 n+1) \frac{\ell-1}{\ell} \\
\phi\left(\ell^{b}\right) & \geq(3 n+1) \frac{\ell-1}{\ell} .
\end{aligned}
$$

Since $\ell$ is an odd prime, $\frac{\ell-1}{\ell} \geq \frac{2}{3}$. This implies

$$
\phi\left(\ell^{b}\right) \geq(3 n+1) \frac{\ell-1}{\ell} \geq 2 n+\frac{2}{3}>2 n .
$$

Now consider $\langle g\rangle$ over the field extension $\overline{\mathbb{F}}_{q}$. The generators of $\langle g\rangle$ have $n$ eigenvalues in $\overline{\mathbb{F}}_{q}$, counting multiplicity. Since $|\langle g\rangle|=\ell^{b}$, the eigenvalues of the generators of $\langle g\rangle$ are $\ell^{b}$ roots of unity with at least one eigenvalue a primitive $\ell^{b}$ root of unity. Eigenvalues, counting multiplicity, are a similarity (and hence conjugacy) invariant. We may therefore determine a minimum number of conjugacy classes of the generators of $\langle g\rangle$ by determining all possible combinations of eigenvalues for generators of $\langle g\rangle$. Let $\zeta$ be an eigenvalue of $g$ which is also a primitive $\ell^{b}$ root of unity. Note that this implies $\zeta^{k}$ is an eigenvalue of $g^{k}$. All generators of $\langle g\rangle$ have the form $g^{k}$ and clearly $g^{k}$ is a generator of $\langle g\rangle$ if and only if $\zeta^{k}$ is a primitive $\ell^{b}$ root of unity. Conversely, any primitive $\ell^{b}$-th root of unity is an eigenvalue for some generator of $\langle g\rangle$. The first thought that one might have is that this gives $\phi\left(\ell^{b}\right)$ roots of unity, however, 
we must consider the possibility that taking $g$ to the $k$ power simply permutes the list of eigenvalues of $g$. Since there are $n$ eigenvalues of $g$, the generators of $g$ have greater than or equal to $\phi\left(\ell^{b}\right) / n>2$ lists of eigenvalues and hence fall into greater than 2 conjugacy classes in $\mathrm{SL}_{n}(q)$. This contradicts our earlier result. We conclude that $\mathrm{SL}_{n}(q)$ cannot be quadratic rational for all but possibly 16 cases. 


\section{4. m-RATional GRoups}

In this chapter, we discuss our main results concerning $m$-rational groups in the ordinary case. Recall the definition of an $m$-rational group.

Definition 4.0.3. Let $m \geq 1$ be an integer. A finite group $G$ is $m$-rational if $[\mathbb{Q}(\chi): \mathbb{Q}] \mid m$ for all $\chi \in \operatorname{Irr}(G)$.

First, we state our main result for quadratic rational groups, i.e. $m=2$.

Theorem 4.0.4. Let $G$ be a finite quadratic rational group. Then a finite non-abelian simple group $S$ can occur as a composition factor of $G$ if and only if $S$ is

- An alternating group $\mathrm{A}_{n}, n \geq 5$,

- One of the 20 sporadic groups from the following set:

$$
\begin{gathered}
\left\{\mathrm{M}_{11}, \mathrm{M}_{12}, \mathrm{M}_{22}, \mathrm{M}_{23}, \mathrm{M}_{24}, \mathrm{~J}_{2}, \mathrm{Co}_{1}, \mathrm{Co}_{2}, \mathrm{Co}_{3},\right. \\
\left.\mathrm{Fi}_{22}, \mathrm{Fi}_{23}, \mathrm{Fi}_{24}^{\prime}, \mathrm{HS}, \mathrm{McL}, \mathrm{He}, \mathrm{Suz}, \mathrm{HN}, \mathrm{Th}, \mathrm{B}, \mathrm{M}\right\}
\end{gathered}
$$

- One of the groups of Lie type from the following set:

$$
\begin{gathered}
\left\{\mathrm{L}_{2}(7), \mathrm{L}_{2}(8), \mathrm{L}_{2}(11), \mathrm{L}_{2}(16), \mathrm{L}_{2}(27), \mathrm{L}_{3}(3), \mathrm{L}_{3}(4), \mathrm{L}_{4}(3),\right. \\
\mathrm{U}_{3}(3), \mathrm{U}_{3}(4), \mathrm{U}_{3}(5), \mathrm{U}_{3}(8), \mathrm{U}_{4}(2), \mathrm{U}_{4}(3), \mathrm{U}_{5}(2), \mathrm{U}_{5}(4), \mathrm{U}_{6}(2), \\
\mathrm{S}_{4}(4), \mathrm{S}_{6}(2), \mathrm{S}_{6}(3), \mathrm{S}_{8}(2), \mathrm{O}_{7}(3), \mathrm{O}_{8}^{+}(2), \mathrm{O}_{8}^{+}(3), \mathrm{O}_{8}^{-}(2), \mathrm{O}_{10}^{-}(2), \\
\left.{ }^{2} \mathrm{E}_{6}(2), \mathrm{F}_{4}(2),{ }^{2} \mathrm{~F}_{4}(2)^{\prime}, \mathrm{G}_{2}(3), \mathrm{G}_{2}(4),{ }^{3} \mathrm{D}_{4}(2)\right\} .
\end{gathered}
$$

The proof of Theorem 4.0.4 uses the Classification of Finite Simple Groups, and the representation theory of finite groups of Lie type [6], [10]. It also requires extensive computations to prove the existence of quadratic rational groups in some cases. Now 
we state our general version of the theorem for all natural numbers $m$. Our notation follows [8] for the non-abelian simple groups, and [25] for the characters of finite groups.

Theorem 4.0.5. Let $G$ be a finite m-rational group. Then any non-abelian composition factor $S$ of $G$ is either an alternating group $\mathrm{A}_{n}, n \geq 5$, a sporadic group, or $S$ belongs to a finite set of groups of Lie type, $\mathcal{F}(m)$, that depends only on $m$.

\subsection{Reduction To Simple Groups}

Throughout this section, $G$ is a (non-solvable) finite group, $\operatorname{Irr}(G)$ is the set of irreducible complex characters of $G$, and $m$ is a natural number. The following result gives a necessary character-theoretic condition for a non-abelian simple group $S$ to occur as a non-abelian composition factor of an $m$-rational group $G$.

Theorem 4.1.1. Suppose that $G$ is an m-rational group and $S$ is a non-abelian composition factor of $G$. Let $\alpha \in \operatorname{Irr}(S)$. Then $[\mathbb{Q}(\alpha): \mathbb{Q}]|m| \operatorname{Out}(S) \mid$. In fact, there is a subgroup $R \leq \operatorname{Out}(S)$ such that the $R$-orbit of $\alpha$ has length $j$ and $[\mathbb{Q}(\alpha): \mathbb{Q}] \mid m j$.

To prove this reduction theorem, we first prove the following lemma.

Lemma 4.1.2. Suppose that $G$ is an m-rational group with non-abelian minimal normal subgroup $N=S_{1} \times \cdots \times S_{n} \cong S^{n}$, where $S=S_{1}$. Then there exists a subgroup $R \leq \operatorname{Out}(S)$ such that for any $\alpha \in \operatorname{Irr}(S)$, the character

$$
\hat{\alpha}=\sum_{i=1}^{j} \alpha_{i}
$$

satisfies $[\mathbb{Q}(\hat{\alpha}): \mathbb{Q}] \mid m$, where $\left\{\alpha_{i} \mid 1 \leq i \leq j\right\}$ is the $R$-orbit of $\alpha$.

Proof. For any $g \in G$, conjugation gives an automorphism of $N$. As $\operatorname{Aut}(N)=$ $\operatorname{Aut}(S)$ \ $\operatorname{Sym}_{n}$, we may consider those elements $g \in G$ that fix $S_{1}$, that is, the 
elements $g \in N_{G}\left(S_{1}\right)$. It is clear that we may naturally identify $N_{G}\left(S_{1}\right) /\left(S_{1} C_{G}\left(S_{1}\right)\right)$ with a subgroup $R \leq \operatorname{Out}(S)$.

Let $\alpha \in \operatorname{Irr}(S)$. Since the $R$-orbit of $1_{S}$ is the singleton set $\left\{1_{S}\right\}$, the result holds for the principal character, and thus we assume $\alpha \neq 1_{S}$. Consider the irreducible character $\beta=\alpha \otimes 1_{S_{2}} \otimes \cdots \otimes 1_{S_{n}} \in \operatorname{Irr}(N)$. Let $\chi \in \operatorname{Irr}(G)$ be an irreducible constituent of $\beta^{G}$. Restricting $\chi$ to $N$, we have $\chi_{N}=e \sum_{i=1}^{t} \beta^{g_{i}}$, where $\beta=\beta^{g_{1}}$, and $\left\{\beta^{g_{i}} \mid 1 \leq i \leq t\right\}$ is the $G$-orbit of $\beta$. Furthermore, $t=\left[G: \operatorname{Stab}_{G}(\beta)\right]$ and $\operatorname{Stab}_{G}(\beta) \leq N_{G}\left(S_{1}\right)$.

Now, we see that for any $g \in N_{G}\left(S_{1}\right)$ and $s \in S$,

$$
\beta^{g}((s, 1, \ldots, 1))=\alpha^{g}(s) .
$$

That is, $\beta^{g}=\alpha^{g} \otimes 1_{S_{2}} \otimes \cdots \otimes 1_{S_{n}}$ for any $g \in N_{G}\left(S_{1}\right)$. Further, for $g \notin N_{G}\left(S_{1}\right)$, we have

$$
\beta^{g}(s, 1, \ldots, 1)=\beta\left(1, \ldots, 1, s^{\prime}, 1, \ldots, 1\right)=\alpha(1) \in \mathbb{Z} .
$$

Writing

$$
\chi_{N}=e\left(\sum_{i=1}^{j} \beta^{g_{i}}+\sum_{i=j+1}^{t} \beta^{g_{i}}\right),
$$

where $g_{1}, \ldots, g_{j} \in N_{G}\left(S_{1}\right)$ and $g_{j+1}, \ldots, g_{t} \notin N_{G}\left(S_{1}\right)$, we see that

$$
\begin{gathered}
\sum_{i=1}^{j} \beta^{g_{i}}((s, 1, \ldots, 1))=\frac{\chi_{N}((s, 1, \ldots, 1))}{e}-\sum_{i=j+1}^{t} \beta^{g_{i}}((s, 1, \ldots, 1)) \\
=\frac{\chi_{N}((s, 1, \ldots, 1))}{e}-(t-j) \alpha(1) \in \mathbb{Q}\left(\chi_{N}\right)
\end{gathered}
$$

for any $s \in S$. However,

$$
\sum_{i=1}^{j} \beta^{g_{i}}((s, 1, \ldots, 1))=\sum_{i=1}^{j} \alpha^{g_{i}}(s) .
$$

Now, we observe that $\left\{\alpha_{i}:=\alpha^{g_{i}} \mid 1 \leq i \leq j\right\}$ is the $R$-orbit of $\alpha$ since $\left\{\beta^{g_{i}} \mid 1 \leq i \leq j\right\}$ is the $N_{G}\left(S_{1}\right)$-orbit of $\beta$. 
Finally, notice that $[\mathbb{Q}(\chi): \mathbb{Q}] \mid m$ since $G$ is $m$-rational, and thus $\left[\mathbb{Q}\left(\chi_{N}\right): \mathbb{Q}\right] \mid m$. Hence $[\mathbb{Q}(\hat{\alpha}): \mathbb{Q}] \mid m$ as needed.

We are now able to prove Theorem 4.1.1.

Proof. Suppose that $G$ is an $m$-rational group and $S$ is a non-abelian composition factor of $G$. As quotients of $m$-rational groups are again $m$-rational, we may assume that $N=S_{1} \times \cdots \times S_{n}$ is a minimal normal subgroup of $G$ with $S=S_{1}$. Let $\alpha \in \operatorname{Irr}(S)$, and define $R \leq \operatorname{Out}(S)$ and $\hat{\alpha}$ as in the previous lemma. Note that $\mathbb{Q}(\hat{\alpha}) \subseteq \mathbb{Q}(\alpha)$. Let $\Gamma=\operatorname{Gal}(\mathbb{Q}(\alpha) / \mathbb{Q}(\hat{\alpha}))$. Then for any $\tau \in \Gamma, \tau(\hat{\alpha})=\hat{\alpha}$, and thus, $\tau$ permutes the constituents $\alpha_{i}$. Further, if $\tau$ fixes $\alpha$, then $\tau(\alpha(g))=\alpha(g)$ for all $g \in S$. Thus $\tau$ acts trivially on $\mathbb{Q}(\alpha)$, and must therefore be the identity element in $\Gamma$. As $\mathbb{Q}(\alpha)=\mathbb{Q}\left(\alpha_{i}\right)$ for all $1 \leq i \leq j, \operatorname{Stab}_{\Gamma}\left(\alpha_{i}\right)=1$ for all $1 \leq i \leq j$. By the orbit-stabilizer theorem, it follows that all $\Gamma$-orbits on $\left\{\alpha_{i} \mid 1 \leq i \leq j\right\}$ have length $|\Gamma|$. Therefore

$$
[\mathbb{Q}(\alpha): \mathbb{Q}(\hat{\alpha})]=|\Gamma||j||R||| \operatorname{Out}(S) \mid .
$$

Since $\hat{\alpha}$ satisfies $[\mathbb{Q}(\hat{\alpha}): \mathbb{Q}] \mid m$, we have $[\mathbb{Q}(\alpha): \mathbb{Q}]|m| \operatorname{Out}(S) \mid$, as desired.

Remark 4.1.3. Of course, the alternating groups occur as composition factors of the symmetric groups, which are rational, and hence $m$-rational for all $m$. Further, we note that for any sporadic group $S,|\operatorname{Out}(S)| \mid 2$, and thus, by Theorem 4.1.1, it suffices to find an irreducible character, $\alpha$, of $S$ and an odd prime number dividing $[\mathbb{Q}(\alpha): \mathbb{Q}]$ to eliminate $S$ as a possible composition factor of a quadratic rational group. Using GAP, we see that $\mathrm{J}_{1}, \mathrm{~J}_{3}, \mathrm{~J}_{4}, \mathrm{Ru}, \mathrm{O}^{\prime} \mathrm{N}$, and Ly each have such an irreducible character. The remaining sporadic groups are quadratic rational.

In light of Theorem 4.1.1, we will attempt to construct irreducible characters of the finite simple groups of Lie type which take on highly irrational values. In particular, given a simple group of Lie type, $S$, we will try to find $\alpha \in \operatorname{Irr}(S)$ satisfying $[\mathbb{Q}(\alpha)$ : $\mathbb{Q}]>m|\operatorname{Out}(S)|$. 
Often, the value $[\mathbb{Q}(\alpha): \mathbb{Q}]$ will be given in terms of the Euler totient function, and we therefore recall the following useful, but well-known lemma that we proved in Chapter 3 (see Lemma 3.1.7).

Lemma 4.1.4. Let $n \geq 2$. Then $\phi(n) \geq \sqrt{\frac{n}{2}}$.

Finally, recall that if $k$ is an integer, then we denote the 2-part of $k$ (i.e. the highest power of 2 dividing $k$ ) by $k_{2}$, and write $k_{2^{\prime}}:=\frac{k}{k_{2}}$, the $2^{\prime}$-part of $k$. In particular, if $a$ and $b$ are integers, then $(a, b)_{2^{\prime}}$ denotes the $2^{\prime}$-part of $(a, b)$, the greatest common divisor of $a$ and $b$. The following lemma will be useful in our computations:

Lemma 4.1.5. Suppose that $q$ is a natural number. Then for any natural number $n$,

$$
\left(q^{n}+1\right)_{2}= \begin{cases}(q+1)_{2} & \text { if } n \text { is odd }, \\ (2, q-1) & \text { if } n \text { is even } .\end{cases}
$$

Proof. See [50, Lemma 5 (i)].

Using GAP, we recognize that a good candidate for our character $\alpha$ in the classical groups of Lie type is the Deligne-Lusztig character associated to a particular torus. We will now give the construction of this character for the projective special linear groups, as it proved useful in learning about the generalized Deligne-Lusztig characters. However, we found that it was difficult to prove that the field of values associated to this character was sufficiently irrational without realizing the character as a semisimple character corresponding to a semisimple element of the dual group. Thus, after this example, we will simply construct the characters $\alpha$ as semisimple characters, where we have techniques of proving that the field of values is sufficiently irrational.

Example 1. Let $\mathcal{T}$ be the usual maximal torus containing diagonal elements in 
$\mathrm{SL}_{n}\left(\overline{\mathbb{F}}_{q}\right)$. Consider the element

$$
w=\left(\begin{array}{ccccc}
0 & 0 & \cdots & 0 & 1 \\
1 & 0 & \cdots & 0 & 0 \\
0 & 1 & \cdots & 0 & 0 \\
\vdots & & \ddots & & \vdots \\
0 & 0 & \cdots & 1 & 0
\end{array}\right) \in \operatorname{SL}_{n}\left(\bar{F}_{q}\right)
$$

By surjectivity of the Lang map ([6], pg. 32), there exists $g \in \mathrm{SL}_{n}\left(\bar{F}_{q}\right)$ such that $g^{-1} F(g)=w$, where $F$ is the standard Frobenius endomorphism. Define the maximal torus $\mathcal{T}_{w}=g \mathcal{T} g^{-1}$. Then $F$ acts on $\mathcal{T}_{w}$ in the same way that $w \cdot F$ acts on $\mathcal{T}$. Therefore, a maximal torus in $\mathrm{SL}_{n}\left(\mathbb{F}_{q}\right)$ is

$$
\begin{aligned}
& T=\mathcal{T}_{w}^{F}=\left\{t=\operatorname{diag}\left(t_{1}, \ldots, t_{n}\right) \mid t_{1}=t_{n}^{q}, t_{2}=t_{1}^{q}, \ldots, t_{n}=t_{n-1}^{q} \text { and } \operatorname{det}(t)=1\right\} \\
& =\left\{\operatorname{diag}\left(t_{1}, t_{1}^{q}, t_{1}^{q^{2}}, \ldots, t_{1}^{q^{n-1}}\right) \mid t_{1}^{q^{n}}=t_{1} \text { and } t_{1}^{1+q+\cdots+q^{n-1}}=1\right\} \cong \mathbb{Z} /\left(\frac{q^{n}-1}{q-1}\right) \mathbb{Z} .
\end{aligned}
$$

Now, let $\epsilon$ be a primitive $\frac{q^{n}-1}{q-1}$-th root of unity, and consider the character $\theta$ of $T=\langle t\rangle$ defined by $\theta(t)=\epsilon^{(n, q-1)}$. We have that $W(T)$, the Weyl group of $T$, is cyclic of order $n$ (see, for example [42]), generated by $w$, and $W(T)$ acts on $\theta$ by $\theta^{w}(t)=\theta\left(w t w^{-1}\right)=(\theta(t))^{q^{n-1}}$. Thus, it is easy to see that $\theta$ is in general position: Suppose that $w^{k} \in \operatorname{Stab}_{W(T)}(\theta)$. Then $\epsilon^{(n, q-1)}=\theta(t)=\theta^{w^{k}}(t)=\epsilon^{(n, q-1) q^{n-k}}$. Hence, we have $\epsilon^{(n, q-1)\left(q^{n-k}-1\right)}=1$, and it follows that

$$
\frac{q^{n}-1}{q-1} \mid(n, q-1)\left(q^{n-k}-1\right) \text {. }
$$

Since $\frac{q^{n}-1}{q-1}>(n, q-1)\left(q^{n-k}-1\right)$ for all $k \geq 2$, we have that $w^{k}$ does not stabilize $\theta$ for $2 \leq k \leq n-1$. Further, it follows that if $n>2, \operatorname{Stab}_{W(T)}(\theta)$ is a proper subgroup of $W(T)$, hence $w \notin \operatorname{Stab}_{W(T)}(\theta)$ since $w$ generates $W(T)$. If $n=2$, we also see that $q+1=\frac{q^{2}-1}{q-1} \nmid(2, q-1)(q-1)$ (since $\left.q>3\right)$, and in this case $w \notin \operatorname{Stab}_{W(T)}(\theta)$. Hence, $\theta$ is in general position. Therefore, we have that the associated Deligne-Lusztig character $\pm R_{T}(\theta)=: \alpha$ is an irreducible character of $\mathrm{SL}_{n}\left(\mathbb{F}_{q}\right)$. Using the formula for $\alpha$ on semisimple elements, Proposition 7.5.3 of []. we see that $\operatorname{ker}(\alpha) \supseteq Z\left(\mathrm{SL}_{n}\left(\mathbb{F}_{q}\right)\right)=$ $\left\langle t^{\frac{q^{n}-1}{(n, q-1)(q-1)}}\right\rangle=:\langle z\rangle$ : 


$$
\begin{aligned}
& R_{T}(\theta)(z)=\frac{\epsilon_{T} \epsilon_{C^{0}(z)}}{|T|\left|C^{0}(z)^{F}\right|_{p}} \sum_{g \in \mathrm{SL}_{n}\left(\mathbb{F}_{q}\right), g^{-1} z g \in T} \theta\left(g^{-1} z g\right)=\frac{\epsilon_{T} \epsilon_{C^{0}(z)}\left|\mathrm{SL}_{n}\left(\mathbb{F}_{q}\right)\right|}{|T|\left|C^{0}(z)^{F}\right|_{p}} \theta(z) \\
= & \frac{\epsilon_{T} \epsilon_{C^{0}(1)}\left|\mathrm{SL}_{n}\left(\mathbb{F}_{q}\right)\right|}{|T|\left|C^{0}(1)^{F}\right|_{p}} \theta(1)=\frac{\epsilon_{T} \epsilon_{C^{0}(1)}}{|T|\left|C^{0}(1)^{F}\right|_{p}} \sum_{g \in \mathrm{SL}_{n}\left(\mathbb{F}_{q}\right), g^{-1} 1 g \in T} \theta\left(g^{-1} 1 g\right)=R_{T}(\theta)(1),
\end{aligned}
$$

since $C^{0}(z)=C^{0}(1)$ and $\theta(z)=\theta(1)$. Therefore, we may view $\alpha$ as an irreducible character of $\operatorname{PSL}_{n}(q)$. Finally, evaluating at $t^{j}$ for all $1 \leq j \leq \frac{q^{n}-1}{q-1}$ with $\left(j, \frac{q^{n}-1}{q-1}\right)=1$, we see that $\alpha$ takes on the values

$$
\begin{aligned}
\alpha\left(t^{j}\right) & = \pm R_{T}(\theta)\left(t^{j}\right)= \pm \frac{\epsilon_{T} \epsilon_{C^{0}\left(t^{j}\right)}}{|T|\left|C^{0}\left(t^{j}\right)^{F}\right|_{p}} \sum_{g \in \mathrm{SL}_{n}\left(\mathbb{F}_{q}\right), g^{-1} t^{j} g \in T} \theta\left(g^{-1} t^{j} g\right) \\
& = \pm \sum_{w \in W(T)} \theta^{w}\left(t^{j}\right)= \pm\left(\epsilon^{d j}+\epsilon^{d j q}+\epsilon^{d j q^{2}}+\cdots+\epsilon^{d j q^{n-1}}\right),
\end{aligned}
$$

for all $1 \leq j \leq \frac{q^{n}-1}{q-1},\left(j, \frac{q^{n}-1}{q-1}\right)=1$, where $d=(n, q-1)$, as $t^{j}$ is regular semisimple, and thus contained in a unique maximal torus.

However, as mentioned above, we were unable to prove that

$\left[\mathbb{Q}\left(\left\{\epsilon^{d j}+\epsilon^{d j q}+\epsilon^{d j q^{2}}+\cdots+\epsilon^{d j q^{n-1}} \mid 1 \leq j \leq \frac{q^{n}-1}{q-1},\left(j, \frac{q^{n}-1}{q-1}\right)=1\right\}\right): \mathbb{Q}\right]=\frac{\phi\left(\frac{q^{n}-1}{(n, q-1)(q-1)}\right)}{n}$,

and therefore we will need to realize this character as a semisimple character. (In fact, in the notation of [23], the semisimple element we choose will correspond to the general position character $\theta \in \operatorname{Irr}(T)$ that we defined above.)

We are now able to prove our main results for the groups of Lie type.

\subsection{The Linear Groups}

Theorem 4.2.1. Let $S=\operatorname{PSL}_{n}(q)=\operatorname{PSL}_{n}\left(p^{f}\right)$, and define $N=\frac{q^{n}-1}{(n, q-1)(q-1)}$.

Then, there exists an irreducible character $\alpha \in \operatorname{Irr}(S)$ with $[\mathbb{Q}(\alpha): \mathbb{Q}]=\frac{\phi(N)}{n}$.

Further, for a fixed $m \in \mathbb{N}$, only finitely many pairs $(n, q)$ satisfy $\frac{\phi(N)}{n}|m| \operatorname{Out}(S) \mid$. 
Hence, $\mathrm{PSL}_{n}(q)$ can occur as a composition factor of an m-rational group for only finitely many pairs $(n, q)$.

As mentioned above, we will need primitive prime divisors to construct $\alpha$. Therefore, we first prove the result for $n=2$, as a primitive prime divisor of $q^{2}-1$ does not necessarily exist, as well as for $\mathrm{PSL}_{6}(2)$.

Lemma 4.2.2. Theorem 4.2.1 holds if $n=2$. Further, $\mathrm{PSL}_{6}(2)$ cannot occur as a composition factor of any quadratic rational group.

Proof. The character table of $\operatorname{PSL}_{2}(q)$ is known in CHEVIE [19], and there exists an irreducible character $\alpha$ of degree $q-1$ which takes on the value $\pm\left(\epsilon+\epsilon^{-1}\right)$, where $\epsilon$ is a primitive $\frac{q^{2}-1}{(2, q-1)(q-1)}$-th root of unity, and is rational on every other conjugacy class. Clearly, $\left[\mathbb{Q}(\epsilon): \mathbb{Q}\left(\epsilon+\epsilon^{-1}\right)\right]=2$, and therefore,

$$
[\mathbb{Q}(\alpha): \mathbb{Q}]=\frac{\phi\left(\frac{q^{2}-1}{(q-1)(2, q-1)}\right)}{2} .
$$

Using the character table of $\mathrm{PSL}_{6}(2)$, we see that there is a character $\alpha$ of degree 19,845 with $[\mathbb{Q}(\alpha): \mathbb{Q}]=6$. However, $2\left|\operatorname{Out}\left(\mathrm{PSL}_{6}(2)\right)\right|=4$ and thus, by Lemma 4.1.1. $\mathrm{PSL}_{6}(2)$ cannot occur as a composition factor of any quadratic rational group.

To prove Theorem 4.2.1 in the general case, we will need the following lemmas, which generalize to the other groups of Lie type. Thus, we will work in the following setup: Let $\mathcal{G}$ be a simple, simply connected algebraic group and $F$ a Frobenius morphism of $\mathcal{G}$ such that $L / Z(L)=S$ is a simple group of Lie type, where $L:=\mathcal{G}^{F}$. Let $L^{*}$ be the dual of $L,[6]$.

Lemma 4.2.3. Suppose $s \in\left[L^{*}, L^{*}\right]$ is semisimple with connected centralizer. Then $\chi_{s}$, the semisimple character of $L$ labeled by $s$ is irreducible over $L$ and is trivial at $Z(L)$, whence it can be viewed as an irreducible character of $S=L / Z(L)$. Moreover, if $\theta$ is a complex primitive $|s|$-th root of unity, then $\mathbb{Q}\left(\chi_{s}\right) \subseteq \mathbb{Q}(\theta)$. 
Proof. See, for example, Lemma 4.4 in [38], and Lemma 9.1 in [40].

Lemma 4.2.4. Let $s$ be as in the previous lemma, and assume $\theta$ is a primitive $o(s)$-th root of unity. Then the Galois automorphism sending $\theta$ to $\theta^{\ell}$ acts trivially on $\mathbb{Q}\left(\chi_{s}\right)$ $\Longleftrightarrow \chi_{s}=\chi_{s} \ell$

Proof. We follow the arguments made in Chapter 9 of [40]. Let $\sigma$ be the Galois automorphism sending $\theta$ to $\theta^{\ell}$, and assume that $\sigma$ acts trivially on $\mathbb{Q}\left(\chi_{s}\right)$. Then $\chi_{s}=\left(\chi_{s}\right)^{\sigma}$. As in the proof of Lemma 9.1 in [40], we have $\left(\chi_{s}\right)^{\sigma}=\chi_{s^{\ell}}$, and thus, $\chi_{s}=\chi_{s^{\ell}}$. Conversely, if $\chi_{s}=\chi_{s^{\ell}}$, then $\chi_{s}=\left(\chi_{s}\right)^{\sigma}$, and $\sigma$ acts trivially on $\mathbb{Q}\left(\chi_{s}\right)$.

Now, let $L=\operatorname{SL}_{n}(q)$ so that $S=L / Z(L)$, and define $L^{*}=\operatorname{PGL}_{n}(q)$. In view of Lemmas 3.1 .2 and 4.2 .2 , we now assume that $n \geq 3$ and $(n, q) \neq(6,2)$; in particular, we may assume the existence of primitive prime divisors of $q^{n}-1$.

Lemma 4.2.5. Let $s$ be a generator of the unique subgroup of index $(n, q-1)$ in a cyclic maximal torus, $T^{*}$ of $L^{*}$ of order $\frac{q^{n}-1}{q-1}$. Then generators of $\langle s\rangle$ lie in $\frac{\phi(N)}{n}$ distinct $L^{*}$-conjugacy classes.

Proof. Choose $r$ to be a primitive prime divisor of $q^{n}-1$, and let $R^{*}$ be a Sylow $r$-subgroup of $L^{*}$. Note that $r|| s \mid$, and we may define $t=s^{|s| / r}$. Then $|t|=r$, and by Lemma 2.4 of [36], $t$ is regular semisimple, and hence, contained in a unique maximal torus of $L^{*}$, [10, Proposition 14.6(ii)]. As $R^{*}$ is the unique Sylow $r$-subgroup of $T^{*}$, it follows that $N_{L^{*}}\left(T^{*}\right) \leq N_{L^{*}}\left(R^{*}\right)$. Further, as $t$ is contained uniquely in $T^{*}$ and $\langle t\rangle=\Omega_{1}\left(R^{*}\right)$ (the subgroup of $R^{*}$ generated by all elements of order $r$ ), we have $N_{L^{*}}\left(R^{*}\right) \leq N_{L^{*}}\left(T^{*}\right)$, and hence equality. Now, suppose that $s^{i}$ and $s^{j}$ are generators of $\langle s\rangle$ that are $L^{*}$-conjugate, say $\left(s^{i}\right)^{h}=s^{j}$. Then

$$
\left(t^{i}\right)^{h}=\left(s^{|s| i / r}\right)^{h}=\left(\left(s^{i}\right)^{h}\right)^{|s| / r}=\left(s^{j}\right)^{|s| / r}=t^{j} .
$$

Hence, by Burnside's Fusion Control Lemma (note that $t^{i}, t^{j} \in Z\left(R^{*}\right)$ ), we have that $h \in N_{L^{*}}\left(R^{*}\right)=N_{L^{*}}\left(T^{*}\right)$. Observe that if $\left(s^{i}\right)^{h}=s^{j}$ for some $h \in N_{L^{*}}\left(T^{*}\right)$, then 
$\left(s^{i}\right)^{h a}=s^{j}$ for all $a \in T^{*}$ as $T^{*}$ is abelian, and thus, conjugacy classes are determined by $W\left(T^{*}\right)$-conjugacy. Since $\langle s\rangle$ is the unique subgroup of $T^{*}$ of order $|s|, N_{L^{*}}\left(T^{*}\right)$ acts on $\langle s\rangle$ and sends $s$ to another generator. Moreover, the stabilizer of a point under this action is $C_{L^{*}}(s)=T^{*}$, and thus the $\phi(N)$ generators of $\langle s\rangle$ lie in $L^{*}$-conjugacy classes of size $\left|W\left(T^{*}\right)\right|=n$ [5. Theorem 2.1], and thus, the generators of $\langle s\rangle$ lie in $\phi(N) / n$ distinct $L^{*}$-conjugacy classes.

We are now able to complete the proof of Theorem 4.2.1:

Proof. Choose $s$ as in Lemma 4.2.5. Since $s$ generates the subgroup of $T^{*}$ of index $(n, q-1)=\left[L^{*}:\left[L^{*}, L^{*}\right]\right]$, it is clear that $s \in\left[L^{*}, L^{*}\right]$. In the notation of the previous lemma, define $\alpha:=\chi_{s}$. From Lemma 4.2.4, the Galois automorphism sending $\theta$ to $\theta^{\ell}$, where $\theta$ is a primitive $N$-th root of unity, acts trivially on $\mathbb{Q}\left(\chi_{s}\right) \Longleftrightarrow \chi_{s}=\chi_{s^{\ell}}$. Now, $\chi_{s}=\chi_{s^{\ell}}$ iff $s$ is conjugate to $s^{\ell} \Longleftrightarrow$ the maps sending $x$ to $x^{\ell}$ acts trivially on the conjugacy classes of generators of $\langle s\rangle$. Note that the group consisting of the maps $\left.x \mapsto x^{\ell}, \ell \in \mathbb{Z} / N \mathbb{Z}\right)^{\times}$, acts transitively on the set of conjugacy classes of generators of $\langle s\rangle$. Thus, by the orbit stabilizer theorem, we have $[\mathbb{Q}(\alpha): \mathbb{Q}]=\frac{\phi(N)}{n}$, since the generators of $\langle s\rangle$ lie in $\frac{\phi(N)}{n}$ conjugacy classes.

Now, let $m \in \mathbb{N}$. We have from [29, Table 5.1.A] that

$$
|\operatorname{Out}(S)|=\left\{\begin{array}{ll}
(2, q-1) f & n=2 \\
2(n, q-1) f & n>2
\end{array} .\right.
$$

From Lemma 3.1.7, we see that

$$
\frac{\phi(N)}{n} \geq \sqrt{\frac{N}{2 n^{2}}} \geq \sqrt{\frac{q^{n-1}}{2 n^{3}}} .
$$

Further, $m|\operatorname{Out}(S)| \leq 2 m(n, q-1) f \leq m q^{2}$. Comparing these, we must determine when

$$
\sqrt{\frac{q^{n-1}}{2 n^{3}}}>m q^{2}
$$

Rewriting this as

$$
\sqrt{\frac{q^{n-5}}{2 n^{3}}} \geq \sqrt{\frac{2^{n-6}}{n^{3}}}>m
$$


it is easy to see that there exists $n_{0} \in \mathbb{N}$ large enough so that $\frac{\phi(N)}{n}>m|\operatorname{Out}(S)|$ for all $n \geq n_{0}$ and $q \geq 2$. In particular, $\operatorname{PSL}_{n}(q)$ cannot occur as a composition factor of an $m$-rational group for any $n \geq n_{0}$ and $q \geq 2$. Now, for $3 \leq n \leq n_{0}$, we see that

$$
\frac{\phi(N)}{n} \geq \sqrt{\frac{N}{2 n^{2}}} \geq \sqrt{\frac{q^{n-1}}{2 n^{3}}},
$$

and $2 m(n, q-1) f \leq 2 \log _{2}(q) n m$. As $\sqrt{\frac{q^{n-1}}{8 n^{5}\left(\log _{2}(q)\right)^{2}}}$ is positive and increasing unboundedly for a fixed $3 \leq n \leq n_{0}$ and $q \geq 3$, there exists $q_{0}$ such that $\sqrt{\frac{q^{n-1}}{8 n^{5}\left(\log _{2}(q)\right)^{2}}}>$ $m$ for all $q \geq q_{0}$, and, in particular, $\frac{\phi(N)}{n}>2 m(n, q-1) f$ for all $q \geq q_{0}$. Therefore, only finitely many groups $\operatorname{PSL}_{n}(q)$ can occur as a composition factor of an $m$-rational group.

Corollary 4.2.6. $\mathrm{PSL}_{n}(q)$ can occur as a composition factor of a quadratic rational group if and only if

$$
\begin{aligned}
& (n, q) \in\{(2,4),(2,5),(2,7),(2,8),(2,9),(2,11), \\
& (2,16),(2,27),(3,2),(3,3),(3,4),(4,2),(4,3)\} .
\end{aligned}
$$

Proof. Fix $m=2$. From Theorem 4.2.1, we must solve the relation $\frac{\phi(N)}{n}|2| \operatorname{Out}(S) \mid$. In the notation of the proof of Theorem 4.2.1, we use a computer to determine that we may choose $n_{0}=22$. Then, for each $3 \leq n \leq 22$, we may choose $q_{0}$ as follows:

\begin{tabular}{|c|c|c|c|c|c|c|c|c|c|c|}
\hline$n$ & 3 & 4 & 5 & 6 & 7 & 8 & 9 & 10 & 11 & 12 \\
\hline$q_{0}$ & 860 & 116 & 42 & 22 & 15 & 11 & 9 & 7 & 6 & 5 \\
\hline
\end{tabular}

\begin{tabular}{|c|c|c|c|c|c|c|c|c|c|c|}
\hline$n$ & 13 & 14 & 15 & 16 & 17 & 18 & 19 & 20 & 21 & 22 \\
\hline$q_{0}$ & 5 & 4 & 4 & 4 & 4 & 4 & 3 & 3 & 3 & 3 \\
\hline
\end{tabular}

Verifying which of these (finitely many) pairs $(n, q)$ satisfy $\frac{\phi(N)}{n}|2| \operatorname{Out}(S) \mid$ (see the Appendix for the complete set of computations), we determine that $S=\operatorname{PSL}_{n}(q)$ 
can occur as a composition factor of a quadratic rational group only if

$$
\begin{gathered}
(n, q) \in\{(2,4),(2,5),(2,7),(2,8),(2,9),(2,11),(2,16),(2,19),(2,23),(2,27),(2,31) \\
(3,2),(3,3),(3,4),(3,7),(3,16),(4,2),(4,3)\}
\end{gathered}
$$

Using GAP, we see that $\mathrm{PSL}_{2}(19), \mathrm{PSL}_{2}(23)$, and $\mathrm{PSL}_{3}(16)$ have characters $\chi_{1}$, $\chi_{2}$, and $\chi_{3}$ which give $\left[\mathbb{Q}\left(\chi_{1}\right): \mathbb{Q}\right]=3,\left[\mathbb{Q}\left(\chi_{2}\right): \mathbb{Q}\right]=5$, and $\left[\mathbb{Q}\left(\chi_{3}\right): \mathbb{Q}\right]=32$, respectively. Hence, these cannot occur as a composition factor of any quadratic rational group, as $2|\operatorname{Out}(S)|=4,4$, and 48 , respectively, each a violation of Lemma 4.1.1.

We see that $\mathrm{PSL}_{2}(31)$ has a character, $\alpha$ of degree 32 which is extendible to Aut $\left(\mathrm{PSL}_{2}(31)\right)$. Thus, in the notation of Lemma 4.1.2, $\hat{\alpha}=\alpha$. Hence, if $\mathrm{PSL}_{2}(31)$ is to occur as a composition factor of a quadratic rational group, it must be the case that $[\mathbb{Q}(\alpha): \mathbb{Q}]=[\mathbb{Q}(\hat{\alpha}): \mathbb{Q}] \leq 2$. However, we see that $4 \mid[\mathbb{Q}(\alpha): \mathbb{Q}]$. Therefore, $\mathrm{PSL}_{2}(31)$ cannot occur as a composition factor of a quadratic rational group.

Similarly, $\mathrm{PSL}_{3}(7)$ has a character $\beta$ of degree 288 that extends to $\mathrm{PSL}_{3}(7) .3$. Thus, the $\operatorname{Out}(S)$-orbit of $\beta$ must have length at most 2 , and hence $[\mathbb{Q}(\beta): \mathbb{Q}] \mid 4$ by Theorem 4.1.1. However, $[\mathbb{Q}(\beta): \mathbb{Q}]=6$, and therefore, $\operatorname{PSL}_{3}(7)$ cannot occur as a composition factor of any quadratic rational group.

Using GAP, we see that the remaining groups $S$ do occur as a composition factor of some quadratic rational (abbreviated QR) group:

\begin{tabular}{|c|c|c|c|c|c|}
\hline$(n, q)$ & $(2,4)$ & $(2,5)$ & $(2,7)$ & $(2,8)$ & $(2,9)$ \\
\hline QR Group & $\mathrm{PSL}_{2}(4)$ & $\mathrm{PSL}_{2}(5)$ & $\mathrm{PSL}_{2}(7)$ & $\mathrm{PSL}_{2}(8) .3$ & $\mathrm{PSL}_{2}(9)$ \\
\hline
\end{tabular}

\begin{tabular}{|c|c|c|c|}
\hline$(n, q)$ & $(2,11)$ & $(2,16)$ & $(2,27)$ \\
\hline QR Group & $\mathrm{PSL}_{2}(11)$ & $\mathrm{PSL}_{2}(16) \cdot 4$ & $\mathrm{PSL}_{2}(27) .3$ \\
\hline
\end{tabular}




\begin{tabular}{|c|c|c|c|c|c|}
\hline$(n, q)$ & $(3,2)$ & $(3,3)$ & $(3,4)$ & $(4,2)$ & $(4,3)$ \\
\hline QR Group & $\mathrm{PSL}_{3}(2)$ & $\mathrm{PSL}_{3}(3) .2$ & $\mathrm{PSL}_{3}(4)$ & $\mathrm{PSL}_{4}(2)$ & $\mathrm{Aut}_{\left(\mathrm{PSL}_{4}(3)\right)}$ \\
\hline
\end{tabular}

Note that $\mathrm{L}_{2}(4) \cong \mathrm{L}_{2}(5) \cong \mathrm{A}_{5}, \mathrm{~L}_{2}(9) \cong \mathrm{A}_{6}, \mathrm{~L}_{3}(2) \cong \mathrm{L}_{2}(7)$, and $\mathrm{L}_{4}(2) \cong \mathrm{A}_{8}$, and thus, we remove $\mathrm{L}_{2}(4), \mathrm{L}_{2}(5), \mathrm{L}_{2}(9), \mathrm{L}_{3}(2)$, and $\mathrm{L}_{4}(2)$ from our list in the statement of Theorem 4.0.4.

\subsection{The Unitary Groups}

Theorem 4.3.1. Let $S=\operatorname{PSU}_{n}(q), q^{2}=p^{f}$. Then $S$ can occur as a composition factor of an m-rational group for only finitely many pairs $(n, q)$.

To prove Theorem 4.1, we must consider the parity of $n$ :

Proposition 4.3.2. Suppose $n$ is even, $(n, q) \neq(4,2)$, and define $N=\frac{q^{n-1}+1}{(n, q+1)}$. Then, there exists an irreducible character $\alpha \in \operatorname{Irr}(S)$ with $[\mathbb{Q}(\alpha): \mathbb{Q}]=\frac{\phi(N)}{n-1}$. Further, for a fixed $m \in \mathbb{N}$, only finitely many pairs $(n, q)$ satisfy $\frac{\phi(N)}{n-1}|m| \operatorname{Out}(S) \mid$. Hence, $\operatorname{PSU}_{n}(q)$ with $n$ even can occur as a composition factor of an m-rational group for only finitely many pairs $(n, q)$.

Proof. The proof is nearly identical to that of the linear groups. Define $s$ to be a generator of the unique subgroup of index $(n, q+1)$ of a cyclic maximal torus $T^{*}$ of order $q^{n-1}+1$ in $L^{*}=\mathrm{PGU}_{n}(q)$, the dual of $L=\mathrm{SU}_{n}(q)$, [5, Theorem 2.2]. Let $r$ be a primitive prime divisor of $q^{2(n-1)}-1$. Following the proof of Lemma 4.2.5, we see that generators of $\langle s\rangle$ lie in $\frac{\phi(N)}{n-1}$ distinct $L^{*}$-conjugacy classes, as $W\left(T^{*}\right) \cong C_{n-1}$ is cyclic of order $n-1$, [5, Theorem 2.2]. Therefore, $[\mathbb{Q}(\alpha): \mathbb{Q}]=\frac{\phi(N)}{n-1}$, where $\alpha:=\chi_{s}$ is the irreducible semisimple character of $S$ labelled by $s$.

As in the linear group case, comparing $\frac{\phi(N)}{n-1}$ to a square root function, we have

$$
\frac{\phi(N)}{n-1} \geq \sqrt{\frac{q^{n-1}}{2 n^{3}}} \geq \sqrt{\frac{2^{n-2}}{n^{3}}} .
$$


Further, we see that $m|\operatorname{Out}(S)|=m(n, q+1) f \leq 2 m n q\left(\right.$ recall that $\left.q^{2}=p^{f}\right)$ [29, Table 5.1.A]. Clearly, there exists $n_{0}$ large enough so that $\sqrt{\frac{2^{n-6}}{n^{5}}}>m$ for all $n \geq n_{0}$, and in particular, $\operatorname{PSU}_{n}(q)$ cannot occur as a composition factor of any $m$-rational group for $n \geq n_{0}(n$ even) and $q \geq 2$.

Now, for a fixed $4 \leq n \leq n_{0}$, we see that $\sqrt{\frac{q^{n-1}}{8 n^{5}\left(\log _{2}(q)\right)^{2}}}$ is positive and increasing unboundedly for all $q \geq 2$. Hence, for each $4 \leq n \leq n_{0}$, there exists $q_{0}$ such that $\sqrt{\frac{q^{n-1}}{8 n^{5}\left(\log _{2}(q)\right)^{2}}}>m$ for all $q \geq q_{0}$, and $\operatorname{PSU}_{n}(q)$ cannot occur as a composition factor of any $m$-rational group for $4 \leq n \leq n_{0}$ even and $q \geq q_{0}$. Therefore, $\operatorname{PSU}_{n}(q)$ with $n$ even can occur as a composition factor of an $m$-rational group for only finitely many pairs $(n, q)$.

Proposition 4.3.3. Suppose $n$ is odd, and define $N=\frac{q^{n}+1}{(n, q+1)(q+1)}$. Then, there exists an irreducible character $\alpha \in \operatorname{Irr}(S)$ with $[\mathbb{Q}(\alpha): \mathbb{Q}]=\frac{\phi(N)}{n}$. Further, for a fixed $m \in \mathbb{N}$, only finitely many pairs $(n, q)$ satisfy $\frac{\phi(N)}{n}|m| \operatorname{Out}(S) \mid$. Hence, $\operatorname{PSU}_{n}(q)$ with $n$ odd can occur as a composition factor of an m-rational group for only finitely many pairs $(n, q)$.

Proof. Define $s$ to be a generator of the unique subgroup of index $(n, q+1)$ of a cyclic maximal torus $T^{*}$ of order $\frac{q^{n}+1}{q+1}$ in $L^{*}=\operatorname{PGU}_{n}(q)$, the dual of $L=\mathrm{SU}_{n}(q)$, [5, Theorem 2.2]. Let $r$ be a primitive prime divisor of $q^{2 n}-1$. Following the proof of Lemma 4.2 .5 , we see that generators of $\langle s\rangle$ lie in $\frac{\phi(N)}{n}$ distinct $L^{*}$-conjugacy classes, as $W\left(T^{*}\right) \cong C_{n}$ is cyclic of order $n,\left[5\right.$, Theorem 2.2]. Therefore, $[\mathbb{Q}(\alpha): \mathbb{Q}]=\frac{\phi(N)}{n}$, where $\alpha:=\chi_{s}$ is the irreducible semisimple character of $S$ labelled by $s$.

As in the linear group case, comparing $\frac{\phi(N)}{n}$ to a square root function, we have

$$
\frac{\phi(N)}{n} \geq \sqrt{\frac{q^{n-2}}{2 n^{3}}} \geq \sqrt{\frac{2^{n-3}}{n^{3}}} .
$$

Further, we see that $m|\operatorname{Out}(S)|=m(n, q+1) f \leq 2 m n q\left(\right.$ recall that $\left.q^{2}=p^{f}\right)$ [29, Table 5.1.A]. Clearly, there exists $n_{0}$ large enough so that $\sqrt{\frac{2^{n-7}}{n^{5}}}>m$ for all $n \geq n_{0}$, 
and in particular, $\operatorname{PSU}_{n}(q)$ cannot occur as a composition factor of any $m$-rational group for $n \geq n_{0}(n$ odd $)$ and $q \geq 2$.

Now, for a fixed $3 \leq n \leq n_{0}$, we see that $\sqrt{\frac{q^{n-2}}{8 n^{5}\left(\log _{2}(q)\right)^{2}}}$ is positive and increasing unboundedly for all $q \geq 8$. Hence, for each $3 \leq n \leq n_{0}$, there exists $q_{0}$ such that $\sqrt{\frac{q^{n-2}}{8 n^{5}\left(\log _{2}(q)\right)^{2}}}>m$ for all $q \geq q_{0}$, and $\operatorname{PSU}_{n}(q)$ cannot occur as a composition factor of any $m$-rational group for $3 \leq n \leq n_{0}$ odd and $q \geq q_{0}$. Therefore, $\operatorname{PSU}_{n}(q)$ with $n$ odd can occur as a composition factor of an $m$-rational group for only finitely many pairs $(n, q)$.

Corollary 4.3.4. $S=\operatorname{PSU}_{n}(q)$ can occur as a composition factor of a quadratic rational group if and only if

$$
(n, q) \in\{(3,3),(3,4),(3,5),(3,8),(4,2),(4,3),(5,2),(5,4),(6,2)\}
$$

Proof. Fix $m=2$. First, as a Zsigmondy prime does not exist for $2^{6}-1$, we note that $\mathrm{U}_{4}(2)$ is quadratic rational. Now, from Propositions 4.3 .2 and 4.3 .3 , we must solve the relations $\frac{\phi\left(\frac{q^{n}+1}{(n, q+1)(q+1)}\right)}{n}|2| \operatorname{Out}(S) \mid$ for $n$ odd and $\frac{\phi\left(\frac{q^{n-1}+1}{(n, q+1)}\right)}{n-1}|2| \operatorname{Out}(S) \mid$ for $n$ even. In the notation of the proofs of Propositions 4.3 .2 and 4.3 .3 , we find that $n_{0}=34$ in the odd case and $n_{0}=33$ in the even case. For each $n \leq n_{0}$, this yields the following values $q_{0}$ :

\begin{tabular}{|c|c|c|c|c|c|c|c|c|c|c|c|c|c|c|c|}
\hline$n$ & 3 & 4 & 5 & 6 & 7 & 8 & 9 & 10 & 11 & 12 & 13 & 14 & 15 & 16 & 17 \\
\hline$q_{0}$ & 3702239 & 116 & 178 & 22 & 27 & 11 & 12 & 7 & 8 & 5 & 6 & 5 & 5 & 4 & 4 \\
\hline
\end{tabular}

\begin{tabular}{|c|c|c|c|c|c|c|c|c|c|c|c|c|c|c|c|c|}
\hline$n$ & 18 & 19 & 20 & 21 & 22 & 23 & 24 & 25 & 26 & 27 & 28 & 29 & 30 & 31 & 32 & 33 \\
\hline$q_{0}$ & 4 & 4 & 3 & 3 & 3 & 3 & 3 & 3 & 3 & 3 & 3 & 3 & 3 & 3 & 3 & 3 \\
\hline
\end{tabular}

Verifying which of these (finitely many) pairs $(n, q) \neq(4,2)$ satisfy $\frac{\phi\left(\frac{q^{n}+1}{(n, q+1)(q+1)}\right)}{n} \mid$ $2|\operatorname{Out}(S)|$ for $n$ odd and $\frac{\phi\left(\frac{q^{n-1}+1}{(n, q+1)}\right)}{n-1}|2| \operatorname{Out}(S) \mid$ for $n$ even, we determine that $S=$ 
$\operatorname{PSU}_{n}(q)$ can occur as a composition factor of a quadratic rational group only if

$$
(n, q) \in\{(3,3),(3,4),(3,5),(3,8),(3,11),(4,2),(4,3),(5,2),(5,4),(6,2),(9,2)\} .
$$

Using GAP, we see that $\operatorname{PSU}_{3}(8)$ and $\operatorname{PSU}_{9}(2)$ have characters $\chi_{1}$ and $\chi_{2}$ which give $8 \mid\left[\mathbb{Q}\left(\chi_{1}\right): \mathbb{Q}\right]$ and $8 \mid\left[\mathbb{Q}\left(\chi_{2}\right): \mathbb{Q}\right]$, respectively. Hence these cannot occur as a composition factor of any quadratic rational group, as $2|\operatorname{Out}(S)|=36$ and 12 , respectively, each a violation of Lemma 4.1.1.

Further, using GAP and MAGMA ${ }^{1}[3]$, we see that the remaining groups occur as composition factors of quadratic rational groups. That is, $\operatorname{PSU}_{n}(q)$ occurs as a composition factor of a quadratic rational group for pairs:

\begin{tabular}{|c|c|c|c|c|}
\hline$(n, q)$ & $(3,3)$ & $(3,4)$ & $(3,5)$ & $(3,8)$ \\
\hline QR Group & $\mathrm{U}_{3}(3)$ & $\mathrm{U}_{3}(4) .4$ & $\mathrm{U}_{3}(5)$ & $\mathrm{U}_{3}(8) .3_{1}$ \\
\hline
\end{tabular}

\begin{tabular}{|c|c|c|c|c|c|}
\hline$(n, q)$ & $(4,2)$ & $(4,3)$ & $(5,2)$ & $(5,4)$ & $(6,2)$ \\
\hline QR Group & $\mathrm{U}_{4}(2)$ & $\mathrm{U}_{4}(3)$ & $\mathrm{U}_{5}(2)$ & $\mathrm{U}_{5}(4) .4$ & $\mathrm{U}_{6}(2)$ \\
\hline
\end{tabular}

\subsection{The Symplectic and Odd-Dimensional Orthogonal Groups}

Theorem 4.4.1. Let $S=\operatorname{PSp}_{2 n}(q)$ with $n \geq 2$ and $(n, q) \neq(3,2)$ or $S=\Omega_{2 n+1}(q)$ with $n>2, q=p^{f}$. Then $S$ can occur as a composition factor of an $m$-rational group for only finitely many pairs $(n, q)$.

Proof. Let $L=\operatorname{Sp}_{2 n}(q)$ (resp., $L=\operatorname{Spin}_{2 n+1}(q)$ ) so that $L^{*}=\mathrm{SO}_{2 n+1}$ (resp., $L^{*}=$ $\left.\operatorname{PCSp}_{2 n}(q)\right)$. Define $s$ to be a generator of the unique subgroup of index $(2, q-1)$ (resp., $\left.\left(q^{n}+1\right)_{2}\right)$ in a cyclic maximal torus $T^{*}$ of order $q^{n}+1$ in $L^{*}$, [5, Theorem 3], [50, Theorem 1]. Let $r$ be a primitive prime divisor of $q^{2 n}-1$. As in the proof of lemma 4.2.5, it follows that generators of $\langle s\rangle$ lie in $\frac{\phi(N)}{2 n}$ distinct $L^{*}$-conjugacy

\footnotetext{
${ }^{1}$ Thanks to Dr. Eamonn O'Brien for computing the character table of $U_{5}(4) .4$ using MAGMA
} 
classes, where $N=\frac{q^{n}+1}{(2, q-1)}\left(\right.$ resp., $\left.N=\frac{q^{n}+1}{\left(q^{n}+1\right)_{2}}\right)$, as $W\left(T^{*}\right) \cong C_{2 n}$, [5. Theorem 3], [50, Theorem 1]. Therefore, $\left[\mathbb{Q}\left(\chi_{s}\right): \mathbb{Q}\right]=\frac{\phi(N)}{2 n}$.

From Lemma 4.1.1, we must determine when

$\frac{\phi(N)}{2 n}|m| \operatorname{Out}(S) \mid=\left\{\begin{array}{ll}2 m(2, q-1) f & q \text { even and } n=2 \\ m(2, q-1) f & \text { else }\end{array} \quad\right.$ (see [29, Table 5.1.A]).

As usual, applying Lemma 3.1.7 and Lemma 4.1.5, we compare the left side to a square root function, allowing us to find $n_{0} \in \mathbb{N}$ such that $\frac{\phi(N)}{2 n}>m|\operatorname{Out}(S)|$ for all $n \geq n_{0}$ and $q \geq 2$ :

$$
\frac{\phi(N)}{2 n \cdot 2(2, q-1) f}=\frac{\phi\left(\frac{q^{n}+1}{(2, q-1)}\right)}{4 n(2, q-1) f} \geq \sqrt{\frac{q^{n}+1}{256 q^{2} n^{2}}} \geq \sqrt{\frac{2^{n-10}}{n^{2}}} \quad \text { when } L=\operatorname{Sp}_{2 n}(q),
$$

and

$\frac{\phi(N)}{2 n \cdot 2(2, q-1) f}=\frac{\phi\left(\frac{q^{n}+1}{\left(q^{n}+1\right)_{2}}\right)}{4 n(2, q-1) f} \geq \sqrt{\frac{q^{n}+1}{128(q+1) q^{2} n^{2}}} \geq \sqrt{\frac{2^{n-11}}{n^{2}}}$ when $L=\operatorname{Spin}_{2 n+1}(q)$.

As in the linear and unitary cases, for each $n<n_{0}$, we may find $q_{0} \in \mathbb{N}$ so that $\frac{\phi(N)}{2 n}>m|\operatorname{Out}(S)|$ for all $q>q_{0}$ :

$$
\frac{\phi(N)}{2 n \cdot 2(2, q-1) f}=\frac{\phi\left(\frac{q^{n}+1}{(2, q-1)}\right)}{2 n \cdot 2(2, q-1) f} \geq \sqrt{\frac{q^{n}}{256 n^{2}\left(\log _{2}(q)\right)^{2}}} \quad \text { when } L=\operatorname{Sp}_{2 n}(q),
$$

and

$$
\frac{\phi(N)}{2 n \cdot 2(2, q-1) f}=\frac{\phi\left(\frac{q^{n}+1}{\left(q^{n}+1\right)_{2}}\right)}{2 n \cdot 2(2, q-1) f} \geq \sqrt{\frac{q^{n-2}}{128 n^{2}\left(\log _{2}(q)\right)^{2}}} \quad \text { when } L=\operatorname{Spin}_{2 n+1}(q)
$$

Thus, $S$ can occur as a composition factor of an $m$-rational group for only finitely many pairs $(n, q)$.

Corollary 4.4.2. $\operatorname{PSp}_{2 n}(q)$ can occur as a composition factor of a quadratic rational group if and only if $(n, q) \in\{(2,2),(2,3),(2,4),(3,2),(3,3),(4,2)\}$, and $\Omega_{2 n+1}(q)$ can occur as a composition factor of a quadratic rational group if and only if $(n, q)=(3,3)$. 
Proof. Fix $m=2$. First, as a Zsigmondy prime does not exist for $2^{6}-1$, we note that $\mathrm{S}_{6}(2)$ is rational. Now, by Theorem 4.4.1, we must determine when

$$
\frac{\phi(N)}{2 n} \mid \begin{cases}4(2, q-1) f & q \text { even and } n=2 \\ 2(2, q-1) f & \text { else }\end{cases}
$$

where $N$ is defined as above. In the notation of the proof of Theorem 4.4.1 (and using the crudest bounds), we determine that $n_{0}=22$, and for each $n<n_{0}$, we obtain the following values $q_{0}$ :

\begin{tabular}{|c|c|c|c|c|c|c|c|c|c|c|c|}
\hline$n$ & 2 & 3 & 4 & 5 & 6 & 7 & 8 & 9 & 10 & 11 & 12 \\
\hline$q_{0}$ & 589 & 2021944 & 887 & 80 & 26 & 13 & 9 & 6 & 5 & 4 & 4 \\
\hline
\end{tabular}

\begin{tabular}{|c|c|c|c|c|c|c|c|c|c|}
\hline$n$ & 13 & 14 & 15 & 16 & 17 & 18 & 19 & 20 & 21 \\
\hline$q_{0}$ & 4 & 3 & 3 & 3 & 3 & 3 & 3 & 3 & 3 \\
\hline
\end{tabular}

Verifying which of these finitely many pairs $(n, q) \neq(3,2)$ satisfy the necessary divisibility relations, we see that the only possible pairs $(n, q)$ lie in the following set:

$$
\{(2,2),(2,3),(2,4),(3,2),(3,3),(4,2),(5,2)\}
$$

To eliminate $(n, q)=(5,2)$, we observe that $\operatorname{Sp}_{10}(2) \cong \Omega_{11}(2)$ has an irreducible character $\chi$ which satisfies $3 \mid[\mathbb{Q}(\chi): \mathbb{Q}]$, whereas $2|\operatorname{Out}(S)|=2$, a violation of Lemma 4.1.1. Using GAP, we see that the remaining groups do occur as a composition factor of some quadratic rational group:

\begin{tabular}{|c|c|c|c|c|c|c|c|}
\hline$(n, q)$ & $(2,2)$ & $(2,3)$ & $(2,4)$ & $(3,2)$ & $(3,3)$ & $(3,3)$ & $(4,2)$ \\
\hline QR Group & $\mathrm{Sp}_{4}(2)^{\prime}$ & $\mathrm{S}_{4}(3)$ & $\mathrm{S}_{4}(4) .2$ & $\mathrm{~S}_{6}(2) \cong \mathrm{O}_{7}(2)$ & $\mathrm{S}_{6}(3)$ & $\mathrm{O}_{7}(3)$ & $\mathrm{S}_{8}(2) \cong \mathrm{O}_{9}(2)$ \\
\hline
\end{tabular}

Note that $\operatorname{Sp}_{4}(2)^{\prime} \cong \mathrm{A}_{6}$ and $\mathrm{S}_{4}(3) \cong \mathrm{U}_{4}(2)$, and thus, we remove $\mathrm{Sp}_{4}(2)^{\prime}$ and $\mathrm{S}_{4}(3)$ from our list in the statement of Theorem 4.0.4. 


\subsection{The Even-Dimensional Orthogonal Groups}

Theorem 4.5.1. Let $S=\mathrm{O}_{2 n}^{+}(q), q=p^{f}$ and $(n, q) \neq(4,2)$. Then $S$ can occur as a composition factor of an m-rational group for only finitely many pairs $(n, q)$.

Proof. Let $L=\operatorname{Spin}_{2 n}^{+}(q)$ so that $L^{*}=\mathrm{PCO}_{2 n}^{+}(q)^{\circ}$. Let $s$ generate a cyclic subgroup of a maximal torus $T^{*} \cong C_{q^{n-1}+1} \times C_{q+1}$ of $L^{*}$ of index $\left(q^{n-1}+1, q+1\right)_{2^{\prime}}\left(q^{n-1}+\right.$ $1)_{2}(q+1)_{2}$, [50, Theorem 1]. Let $r$ be a primitive prime divisor of $q^{2(n-1)}-1$. Then, following the proof of Lemma 4.2.5, we see that generators of $\langle s\rangle$ lie in $\frac{\phi(N)}{2(n-1)}$ distinct $L^{*}$-conjugacy classes, where $N=\frac{\left(q^{n-1}+1\right)(q+1)}{\left(q^{n-1}+1, q+1\right)_{2^{\prime}}\left(q^{n-1}+1\right)_{2}(q+1)_{2}}$, as $W\left(T^{*}\right) \cong C_{2(n-1)},[50$, Theorem 1]. Therefore, it follows that $\left[\mathbb{Q}\left(\chi_{s}\right): \mathbb{Q}\right]=\frac{\phi(N)}{2(n-1)}$.

Using Lemmas 3.1.7 and 4.1.5, and the formula for $|\operatorname{Out}(S)|$ given in [29, Table 5.1.A], we determine that there exists an $n_{0} \in \mathbb{N}$ such that

$$
\frac{\phi(N)}{2(n-1)}>m|\operatorname{Out}(S)|= \begin{cases}6 m(2, q-1)^{2} f & n=4 \\ 2 m(2, q-1)^{2} f & n \geq 6, n \text { even } \\ 2 m\left(4, q^{n}-1\right) f & n \geq 5, n \text { odd }\end{cases}
$$

for all $n \geq n_{0}$ and $q \geq 2$ :

$$
\frac{\phi(N)}{2(n-1)|\operatorname{Out}(S)|} \geq \sqrt{\frac{q^{n-5}}{4608(n-1)^{2}}} \geq \sqrt{\frac{2^{n-17}}{9(n-1)^{2}}} .
$$

Further, for each $4 \leq n \leq n_{0}$, there exists $q_{0} \in \mathbb{N}$ such that $\frac{\phi(N)}{2(n-1)}>m|\operatorname{Out}(S)|$ for all $q \geq q_{0}$ :

$$
\frac{\phi(N)}{2(n-1)|\operatorname{Out}(S)|} \geq \sqrt{\frac{q^{n-3}}{4608(n-1)^{2}\left(\log _{2}(q)\right)^{2}}} .
$$

Therefore,

$$
\frac{\phi(N)}{2(n-1)}|m| \operatorname{Out}(S) \mid
$$

for only finitely many pairs $(n, q)$, and $S$ can occur as a composition factor of an $m$-rational group for only finitely many pairs $(n, q)$.

Corollary 4.5.2. Let $S=\mathrm{O}_{2 n}^{+}(q), q=p^{f}$. Then $S$ can occur as a composition factor of a quadratic rational group if and only if $(n, q) \in\{(4,2),(4,3)\}$. 
Proof. Fix $m=2$. First, as a Zsigmondy prime does not exist for $2^{6}-1$, we note that $O_{8}^{+}(2)$ is rational. Now, we must determine which pairs $(n, q) \neq(4,2)$ satisfy the divisibility relation

$$
\frac{\phi(N)}{2(n-1)} \mid \begin{cases}12(2, q-1)^{2} f & n=4 \\ 4(2, q-1)^{2} f & n \geq 6, n \text { even } \\ 4\left(4, q^{n}-1\right) f & n \geq 5, n \text { odd }\end{cases}
$$

obtained in Theorem 4.5.1. In the notation of the proof of Theorem 4.5.1, we see that $n_{0}=33$, and for each $4 \leq n<n_{0}$, we give $q_{0}$ in the following table:

\begin{tabular}{|c|c|c|c|c|c|c|c|c|c|c|c|c|c|c|c|}
\hline$n$ & 4 & 5 & 6 & 7 & 8 & 9 & 10 & 11 & 12 & 13 & 14 & 15 & 16 & 17 & 18 \\
\hline$q_{0}$ & 119428262 & 6929 & 319 & 71 & 30 & 17 & 11 & 8 & 7 & 6 & 5 & 4 & 4 & 4 & 3 \\
\hline
\end{tabular}

\begin{tabular}{|c|c|c|c|c|c|c|c|c|c|c|c|c|c|c|}
\hline$n$ & 19 & 20 & 21 & 22 & 23 & 24 & 25 & 26 & 27 & 28 & 29 & 30 & 31 & 32 \\
\hline$q_{0}$ & 3 & 3 & 3 & 3 & 3 & 3 & 3 & 3 & 3 & 3 & 3 & 3 & 3 & 3 \\
\hline
\end{tabular}

Verifying which of the (finitely many) remaining pairs $(n, q) \neq(4,2)$ satisfy the necessary divisibility relation, we see that $S$ can occur as a composition factor of an quadratic rational group only if

$$
(n, q) \in\{(4,2),(4,3),(4,4),(4,5),(4,9),(5,2),(6,2)\}
$$

However, we are able to construct other irreducible semisimple characters that do not satisfy the conclusions of Theorem 4.1.1, allowing us to eliminate $\mathrm{O}_{8}^{+}(4)$, $\mathrm{O}_{8}^{+}(5), \mathrm{O}_{8}^{+}(9), \mathrm{O}_{10}^{+}(2)$, and $\mathrm{O}_{12}^{+}(2)$ as possible composition factors of quadratic rational groups, [50, Theorem 1]:

\begin{tabular}{|c|c|c|c|}
\hline Group $S$ & $\mathrm{O}_{8}^{+}(4)$ & $\mathrm{O}_{8}^{+}(5)$ & $\mathrm{O}_{8}^{+}(9)$ \\
\hline Order $|s|$ & $4^{4}-1=255$ & $\frac{\left(5^{4-1}-1\right)(5-1)}{\left(5^{4-1}-1\right)_{2}(5-1)_{2}}=31$ & $\frac{9^{4}-1}{\left(9^{4}-1\right)_{2}}=205$ \\
\hline Field Degree & $16=\frac{\phi(|s|)}{8} \mid\left[\mathbb{Q}\left(\chi_{s}\right): \mathbb{Q}\right]$ & $5=\frac{\phi(|s|)}{6} \mid\left[\mathbb{Q}\left(\chi_{s}\right): \mathbb{Q}\right]$ & $20=\frac{\phi(|s|)}{8} \mid\left[\mathbb{Q}\left(\chi_{s}\right): \mathbb{Q}\right]$ \\
\hline $2|\operatorname{Out}(S)|$ & 24 & 48 & 96 \\
\hline
\end{tabular}




\begin{tabular}{|c|c|c|}
\hline Group $S$ & $\mathrm{O}_{10}^{+}(2)$ & $\mathrm{O}_{12}^{+}(2)$ \\
\hline Order $|s|$ & $2^{5}-1=31$ & $2^{6-1}-1=31$ \\
\hline Field Degree & $6=\frac{\phi(|s|)}{5} \mid\left[\mathbb{Q}\left(\chi_{s}\right): \mathbb{Q}\right]$ & $3=\frac{\phi(|s|)}{10} \mid\left[\mathbb{Q}\left(\chi_{s}\right): \mathbb{Q}\right]$ \\
\hline $2 \mid$ Out $(S) \mid$ & 4 & 4 \\
\hline
\end{tabular}

Using GAP, we have that the remaining possibilities do, in fact, occur as composition factors of quadratic rational groups:

\begin{tabular}{|c|c|c|}
\hline Group & $\mathrm{O}_{8}^{+}(2)$ & $\mathrm{O}_{8}^{+}(3)$ \\
\hline QR Group & $\mathrm{O}_{8}^{+}(2)$ & $\mathrm{O}_{8}^{+}(3)$ \\
\hline
\end{tabular}

Theorem 4.5.3. Let $S=\mathrm{O}_{2 n}^{-}(q), q^{2}=p^{f}$. Then $S$ can occur as a composition factor of an m-rational group for only finitely many pairs $(n, q)$.

Proof. Let $L=\operatorname{Spin}_{2 n}^{-}(q)$ so that $L^{*}=\operatorname{PCO}_{2 n}^{-}(q)^{\circ}$. Let $s$ generate the unique subgroup of a cyclic maximal torus $T^{*} \cong C_{q^{n}+1}$ in $L^{*}$ of index $\left(q^{n}+1\right)_{2}$, [50, Theorem 1]. Let $r$ be a primitive prime divisor of $q^{2 n}-1$. Following the proof of Lemma 4.2.5, we see that generators of $\langle s\rangle$ lie in $\frac{\phi(N)}{n} L^{*}$-conjugacy classes, as $W\left(T^{*}\right) \cong C_{n}$ is cyclic of order $n,\left[50\right.$, Theorem 1]. Hence, $\left[\mathbb{Q}\left(\chi_{s}\right): \mathbb{Q}\right]=\frac{\phi(N)}{n}$, where $N=\frac{q^{n}+1}{\left(q^{n}+1\right)_{2}}$.

Now, using Lemmas 3.1.7 and 4.1.5, it is easy to see that there exists $n_{0} \in \mathbb{N}$ such that $\frac{\phi(N)}{n}>m\left(4, q^{n}+1\right) f=m|\operatorname{Out}(S)|$ for all $n \geq n_{0}$ and $q \geq 2$, where $|\operatorname{Out}(S)|$ is given in [29, Table 5.1.A]:

$$
\frac{\phi(N)}{\left(4, q^{n}+1\right) f n}=\frac{\phi\left(\frac{q^{n}+1}{\left(q^{n}+1\right)_{2}}\right)}{\left(4, q^{n}+1\right) f n} \geq \sqrt{\frac{q^{n}+1}{64 q^{2} n^{2}\left(q^{n}+1\right)_{2}}} \geq \sqrt{\frac{2^{n-11}}{n^{2}}} .
$$

Further, for each $4 \leq n<n_{0}$, there exists $q_{0}$ such that $\frac{\phi(N)}{n}>m\left(4, q^{n}+1\right) f$ for all $q \geq q_{0}$ :

$$
\frac{\phi\left(\frac{q^{n}+1}{\left(q^{n}+1\right)_{2}}\right)}{\left(4, q^{n}+1\right) f n} \geq \sqrt{\frac{q^{n}+1}{128\left(\log _{2}(q)\right)^{2} n^{2}\left(q^{n}+1\right)_{2}}} \geq \sqrt{\frac{q^{n-2}}{128\left(\log _{2}(q)\right)^{2} n^{2}}} .
$$


Therefore, only finitely many pairs $(n, q)$ can satisfy $\frac{\phi(N)}{n} \mid m\left(4, q^{n}+1\right) f$, and in particular, $S$ can occur as a composition factor of an $m$-rational group for only finitely pairs $(n, q)$.

Corollary 4.5.4. Let $S=\mathrm{O}_{2 n}^{-}(q), q^{2}=p^{f}$. Then $S$ can occur as a composition factor of a quadratic rational group if and only if $(n, q) \in\{(4,2),(5,2)\}$.

Proof. Fix $m=2$. From Theorem 4.5.3, we must determine when $\frac{\phi(N)}{n} \mid 2\left(4, q^{n}+\right.$ 1) $f$. In the notation of the proof of Theorem 4.5.3, we calculate that $n_{0}=22$, and for each $4 \leq n<n_{0}, q_{0}$ is given in the following table:

\begin{tabular}{|c|c|c|c|c|c|c|c|c|c|}
\hline$n$ & 4 & 5 & 6 & 7 & 8 & 9 & 10 & 11 & 12 \\
\hline$q_{0}$ & 887 & 80 & 26 & 13 & 9 & 6 & 5 & 4 & 4 \\
\hline
\end{tabular}

\begin{tabular}{|c|c|c|c|c|c|c|c|c|c|}
\hline$n$ & 13 & 14 & 15 & 16 & 17 & 18 & 19 & 20 & 21 \\
\hline$q_{0}$ & 4 & 3 & 3 & 3 & 3 & 3 & 3 & 3 & 3 \\
\hline
\end{tabular}

Verifying which of these finitely many pairs $(n, q)$ satsify the necessary divisibility condition, we see that $S$ can occur as a composition factor of a quadratic rational group only if $(n, q) \in\{(4,2),(5,2)\}$. Further, using GAP, we see that both do, in fact, occur as a composition factor of some quadratic rational group, namely:

\begin{tabular}{|c|c|c|}
\hline Group & $\mathrm{O}_{8}^{-}(2)$ & $\mathrm{O}_{10}^{-}(2)$ \\
\hline QR Group & $\mathrm{O}_{8}^{-}(2) \cdot 2$ & $\mathrm{O}_{10}^{-}(2) \cdot 2$ \\
\hline
\end{tabular}

\subsection{The Exceptional Groups}

Let $\mathcal{L}$ be a simple simply connected algebraic group and $F$ a Frobenius map on $\mathcal{L}$ so that $L:=\mathcal{L}^{F}$ and $S=L / Z(L)$ is a simple exceptional group of Lie type. In all cases, we choose a regular semisimple element $s \in\left[L^{*}, L^{*}\right]$ with connected centralizer 
to construct $\chi_{s} \in \operatorname{Irr}(S)$. Then, selecting a primitive prime divisor $r$ and calculating the number of conjugacy classes that generators of $\langle s\rangle$ lie in, we obtain a divisibility relation that must be satisfied in order for $S$ to be realizable as a composition factor of an $m$-rational group. We summarize our choices of tori and elements $s$ in the following table, [49, Table 1]:

\begin{tabular}{|c|c|c|}
\hline Group & Cyclic Maximal Torus Order & $|s|$ \\
\hline$E_{6}(q), q=p^{f}$ & $q^{6}+q^{3}+1$ & $\frac{q^{6}+q^{3}+1}{(3,-1)}$ \\
\hline$E_{7}(q), q=p^{f}$ & $\left(q^{6}-q^{3}+1\right)(q+1)$ & $\frac{\left(q^{6}-q^{3}+1\right)(q+1)}{(2, q-1)}$ \\
\hline$E_{8}(q), q=p^{f}$ & $q^{8}+q^{7}-q^{5}-q^{4}-q^{3}+q+1$ & $q^{8}+q^{7}-q^{5}-q^{4}-q^{3}+q+1$ \\
\hline$F_{4}(q), q=p^{f}$ & $q^{4}-q^{2}+1$ & $q^{4}-q^{2}+1$ \\
\hline$G_{2}(q), q=p^{f}$ & $q^{2}-q+1$ & $q^{2}-q+1$ \\
\hline${ }^{2} B_{2}(q), q=2^{f}$ & $q \pm \sqrt{2 q}+1$ & $q \pm \sqrt{2 q}+1$ \\
\hline${ }^{3} D_{4}(q), q^{3}=p^{f}$ & $q^{4}-q^{2}+1$ & $q^{4}-q^{2}+1$ \\
\hline${ }^{2} E_{6}(q), q^{2}=p^{f}$ & $q^{6}-q^{3}+1$ & $\frac{q^{6}-q^{3}+1}{(3, q+1)}$ \\
\hline${ }^{2} F_{4}(q), q=2^{f}$ & $q^{2}+q+1 \pm\left(\sqrt{2 q^{3}}+\sqrt{2 q}\right)$ & $q^{2}+q+1 \pm\left(\sqrt{2 q^{3}}+\sqrt{2 q}\right)$ \\
\hline${ }^{2} G_{2}(q), q=3^{f}$ & $q \pm \sqrt{3 q}+1$ & $q \pm \sqrt{3 q}+1$ \\
\hline
\end{tabular}

Choosing the appropriate primitive prime divisors and following the proof of Lemma 4.2.5. we obtain the divisibility relations stated in the following table, where $|\operatorname{Out}(S)|$ is taken from [29, Table 5.1.B]:

\begin{tabular}{|c|c|c|}
\hline Group & $r$ a P.P.D. of & Divisibility Relation \\
\hline$E_{6}(q), q=p^{f}$ & $q^{9}-1$ & $\frac{\phi(|s|)}{9} \mid 2 m(3, q-1) f$ \\
\hline$E_{7}(q), q=p^{f}$ & $q^{18}-1$ & $\frac{\phi(|s|)}{18} \mid m(2, q-1) f$ \\
\hline$E_{8}(q), q=p^{f}$ & $q^{30}-1$ & $\frac{\phi(|s|)}{30} \mid m f$ \\
\hline$F_{4}(q), q=p^{f}$ & $q^{12}-1$ & $\frac{\phi(|s|)}{12} \mid m(2, q) f$ \\
\hline$G_{2}(q), q=p^{f}$ & $q^{6}-1$ & $\frac{\phi(|s|)}{6} \mid 2 m f$ \\
\hline${ }^{2} B_{2}(q), q=2^{f}$ & $q^{4}-1$ & $\frac{\phi(|s|)}{4} \mid m f$ \\
\hline${ }^{3} D_{4}(q), q^{3}=p^{f}$ & $q^{12}-1$ & $\frac{\phi(|s|)}{4} \mid m f$ \\
\hline${ }^{2} E_{6}(q), q^{2}=p^{f}$ & $q^{18}-1$ & $\frac{\phi(|s|)}{9} \mid m(3, q+1) f$ \\
\hline${ }^{2} F_{4}(q), q=2^{f}$ & $q^{12}-1$ & $\frac{\phi(|s|)}{12} \mid m f$ \\
\hline${ }^{2} G_{2}(q), q=3^{f}$ & $q^{6}-1$ & $\frac{\phi(|\vec{s}|)}{6} \mid m f$ \\
\hline
\end{tabular}


Using the divisbility relations stated in the above table, it is clear from Lemma 3.1 .7 that we have the following theorem:

Theorem 4.6.1. For a fixed integer $m$, only finitely many simple exceptional groups of Lie type can occur as a composition factor of some m-rational group.

Corollary 4.6.2. Suppose $S$ is an exceptional group of Lie type. Then $S$ can occur as a composition factor of some quadratic rational group if and only if

$$
S \in\left\{\mathrm{F}_{4}(2), \mathrm{G}_{2}(2)^{\prime}, \mathrm{G}_{2}(3), \mathrm{G}_{2}(4),{ }^{3} \mathrm{D}_{4}(2),{ }^{2} \mathrm{E}_{6}(2),{ }^{2} \mathrm{~F}_{4}(2)^{\prime},{ }^{2} \mathrm{G}_{2}(3)^{\prime}\right\}
$$

Proof. Fix $m=2$. Solving the divisibility relations in the above table, we see that $S$ can occur as a composition factor of a quadratic rational group only if

$$
\begin{gathered}
S \in\left\{\mathrm{F}_{4}(2), \mathrm{G}_{2}(2)^{\prime}, \mathrm{G}_{2}(3), \mathrm{G}_{2}(4), \mathrm{G}_{2}(5), \mathrm{G}_{2}(8)\right. \\
\left.{ }^{2} \mathrm{~B}_{2}(8),{ }^{2} \mathrm{~B}_{2}(32),{ }^{3} \mathrm{D}_{4}(2),{ }^{2} \mathrm{E}_{6}(2),{ }^{2} \mathrm{~F}_{4}(2),{ }^{2} \mathrm{G}_{2}(3),,{ }^{2} \mathrm{G}_{2}(27)\right\}
\end{gathered}
$$

\begin{tabular}{|c|c|c|c|c|c|c|}
\hline Group $S$ & \multicolumn{2}{|r|}{$\mathrm{G}_{2}(5)$} & \multicolumn{2}{|r|}{$\mathrm{G}_{2}(8)$} & \multicolumn{2}{|r|}{${ }^{2} \mathrm{~B}_{2}(32)$} \\
\hline Field Degree & 5 & {$[\mathbb{Q}(\chi): \mathbb{Q}]$} & 12 & {$[\mathbb{Q}(\chi): \mathbb{Q}]$} & 15 & {$[\mathbb{Q}(\chi): \mathbb{Q}]$} \\
\hline $2|\operatorname{Out}(S)|$ & & 2 & & 6 & & 10 \\
\hline
\end{tabular}

To eliminate $\mathrm{G}_{2}(5), \mathrm{G}_{2}(8)$, and ${ }^{2} \mathrm{~B}_{2}(32)$, we use GAP to find a different irreducible character $\chi$ of $S$ which does not satisfy the conclusions of Lemma 4.1.1:

However, eliminating ${ }^{2} \mathrm{~B}_{2}(8)$ and ${ }^{2} \mathrm{G}_{2}(27)$ requires more work. First, suppose that $S={ }^{2} \mathrm{~B}_{2}(8)$. Suppose that $G$ is a quadratic rational group with minimal normal subgroup $N=S_{1} \times \cdots \times S_{n} \cong S^{n}$. Then, $N_{G}\left(S_{1}\right) / C_{G}\left(S_{1}\right) \cong S$ or $N_{G}\left(S_{1}\right) / C_{G}\left(S_{1}\right) \cong$ S.3. If we are in the first case, we consider the irreducible character $\chi$ of $S$ of degree 65 . In this case, $[\mathbb{Q}(\chi): \mathbb{Q}]=3$, and in particular, there exists some $g=(s, 1, \ldots, 1) \in G$ such that $\chi(s)$ has a degree 3 minimal polynomial over $\mathbb{Q}$. Inflating this character to $\hat{\chi} \in \operatorname{Irr}\left(N_{G}\left(S_{1}\right)\right)=\operatorname{Irr}\left(I_{G}(\chi)\right)$ and inducing to $G$ yields an irreducible character 
$\hat{\chi}^{G}=\psi$ of $G$ by the Clifford correspondence. Let $T$ be a transversal of $N_{G}\left(S_{1}\right)$ in $G$. Then by the formula for the induced character, we have $\left.\psi\right|_{N_{G}\left(S_{1}\right)}=\sum_{t \in T} \hat{\chi}^{t}$. In particular, evaluating at $g$, we see that $\psi(g)=\chi(s)+(|T|-1) \chi(1)$. Hence, $3 \mid[\mathbb{Q}(\psi(g)): \mathbb{Q}]$, and $G$ is not quadratic rational. Thus, it must be the case that $N_{G}\left(S_{1}\right) / C_{G}\left(S_{1}\right) \cong S .3$. Let $\chi$ be the irreducible character of $S$ of degree 14 that extends to a non-quadratic-rational character $\psi$ of $S .3$. Since $\psi$ restricts irreducibly to $S$, we have that $\psi^{G} \in \operatorname{Irr}(G)$ by the Clifford correspondence. We see that $\mathbb{Q}(\psi)=$ $\mathbb{Q}(i, \sqrt{3})$ and $\mathbb{Q}(\chi)=\mathbb{Q}(i)$. Let $\sigma$ be the nontrivial element of $\operatorname{Gal}(\mathbb{Q}(i, \sqrt{3}) / \mathbb{Q}(i))$. Then $\psi$ is not fixed by $\sigma$, and therefore, from the formula for induced characters as above, $\psi^{G}$ is not fixed by $\sigma$. Thus, $\mathbb{Q}\left(\psi^{G}\right) \supseteq \mathbb{Q}(i, \sqrt{3})$, and it cannot be the case that $G$ is quadratic rational.

Now, suppose that $S={ }^{2} \mathrm{G}_{2}(27)$. Suppose that $G$ is a quadratic rational group with minimal normal subgroup $N=S_{1} \times \cdots \times S_{n} \cong S^{n}$. Then, $N_{G}\left(S_{1}\right) / C_{G}\left(S_{1}\right) \cong S$ or $N_{G}\left(S_{1}\right) / C_{G}\left(S_{1}\right) \cong S .3$. If we are in the first case, we consider an irreducible character $\chi$ of $S$ of degree 18278. In this case, $[\mathbb{Q}(\chi): \mathbb{Q}]=3$, and in particular, there exists some $g=(s, 1, \ldots, 1) \in G$ such that $\chi(s)$ has a degree 3 minimal polynomial over $\mathbb{Q}$. Inflating this character to $\hat{\chi} \in \operatorname{Irr}\left(N_{G}\left(S_{1}\right)\right)=\operatorname{Irr}\left(I_{G}(\chi)\right)$ and inducing to $G$ yields an irreducible character $\hat{\chi}^{G}=\psi$ of $G$ by the Clifford correspondence. Let $T$ be a transversal of $N_{G}\left(S_{1}\right)$ in $G$. Then by the formula for the induced character, we have $\left.\psi\right|_{N_{G}\left(S_{1}\right)}=\sum_{t \in T} \hat{\chi}^{t}$. In particular, evaluating at $g$, we see that $\psi(g)=\chi(s)+(|T|-$ 1) $\chi(1)$. Hence, $3 \mid[\mathbb{Q}(\psi(g)): \mathbb{Q}]$, and $G$ is not quadratic rational. Thus, it must be the case that $N_{G}\left(S_{1}\right) / C_{G}\left(S_{1}\right) \cong S .3$. Let $\chi$ be the irreducible character of $S$ of degree 2184 that extends to a non-quadratic-rational character $\psi$ of S.3. Since $\psi$ restricts irreducibly to $S$, we have that $\psi^{G} \in \operatorname{Irr}(G)$ by the Clifford correspondence. We see that $\mathbb{Q}(\psi)=\mathbb{Q}\left(-e^{4 i \pi / 9}+e^{10 i \pi / 9}, \sqrt{-3}\right)$ and $\mathbb{Q}(\chi)=\mathbb{Q}(\sqrt{-3})$. Let $\sigma$ be a nontrivial element of $\operatorname{Gal}\left(\mathbb{Q}\left(-e^{4 i \pi / 9}+e^{10 i \pi / 9}, \sqrt{-3}\right) / \mathbb{Q}(\sqrt{-3})\right)$. Then $\psi$ is not fixed by $\sigma$, and therefore, from the formula for induced characters as above, $\psi^{G}$ is not fixed 
by $\sigma$. Thus, $\mathbb{Q}\left(\psi^{G}\right) \supseteq \mathbb{Q}\left(-e^{4 i \pi / 9}+e^{10 i \pi / 9}, \sqrt{-3}\right)$, and it cannot be the case that $G$ is quadratic rational.

The remaining groups do, in fact, occur as a composition factor of some quadratic rational group, which we summarize below:

\begin{tabular}{|c|c|c|c|c|c|c|c|c|}
\hline Group & $\mathrm{F}_{4}(2)$ & $\mathrm{G}_{2}(2)^{\prime}$ & $\mathrm{G}_{2}(3)$ & $\mathrm{G}_{2}(4)$ & ${ }^{3} \mathrm{D}_{4}(2)$ & ${ }^{2} \mathrm{E}_{6}(2)$ & ${ }^{2} \mathrm{~F}_{4}(2)$ & ${ }^{2} \mathrm{G}_{2}(3)^{\prime}$ \\
\hline QR Group & $\mathrm{F}_{4}(2)$ & $\mathrm{G}_{2}(2)^{\prime}$ & $\mathrm{G}_{2}(3)$ & $\mathrm{G}_{2}(4)$ & ${ }^{3} \mathrm{D}_{4}(2) .3$ & ${ }^{2} \mathrm{E}_{6}(2)$ & ${ }^{2} \mathrm{~F}_{4}(2)^{\prime}$ & ${ }^{2} \mathrm{G}_{2}(3)^{\prime} .3$ \\
\hline
\end{tabular}

Note that $\mathrm{G}_{2}(2)^{\prime} \cong \mathrm{U}_{3}(3)$ and ${ }^{2} \mathrm{G}_{2}(3)^{\prime} \cong \mathrm{L}_{2}(8)$, and thus, we remove $\mathrm{G}_{2}(2)^{\prime}$ and ${ }^{2} \mathrm{G}_{2}(3)^{\prime}$ from our list in the statement of Theorem 4.0.4.

\subsection{The Main Results}

We are now able to prove our main results.

Theorem 4.7.1. Let $G$ be a finite m-rational group. Then any non-abelian composition factor $S$ of $G$ is either an alternating group $\mathrm{A}_{n}, n \geq 5$, a sporadic group, or $S$ belongs to a finite set of groups of Lie type, $\mathcal{F}(m)$, that depends only on $m$.

Proof. This follows immediately from Remark 4.1 .3 and Theorems 4.2.1, 4.3.1, 4.4.1, $4.5 .1,4.5 .3,4.6 .1$.

Theorem 4.7.2. Let $G$ be a finite quadratic rational group. Then a finite non-abelian simple group $S$ can occur as a composition factor of $G$ if and only if $S$ is

- An alternating group $\mathrm{A}_{n}, n \geq 5$,

- One of the 20 sporadic groups from the following set:

$$
\begin{gathered}
\left\{\mathrm{M}_{11}, \mathrm{M}_{12}, \mathrm{M}_{22}, \mathrm{M}_{23}, \mathrm{M}_{24}, \mathrm{~J}_{2}, \mathrm{Co}_{1}, \mathrm{Co}_{2}, \mathrm{Co}_{3},\right. \\
\left.\mathrm{Fi}_{22}, \mathrm{Fi}_{23}, \mathrm{Fi}_{24}^{\prime}, \mathrm{HS}, \mathrm{McL}, \mathrm{He}, \mathrm{Suz}, \mathrm{HN}, \mathrm{Th}, \mathrm{B}, \mathrm{M}\right\}
\end{gathered}
$$


- One of the groups of Lie type from the following set:

$$
\begin{gathered}
\left\{\mathrm{L}_{2}(7), \mathrm{L}_{2}(8), \mathrm{L}_{2}(11), \mathrm{L}_{2}(16), \mathrm{L}_{2}(27), \mathrm{L}_{3}(3), \mathrm{L}_{3}(4), \mathrm{L}_{4}(3),\right. \\
\mathrm{U}_{3}(3), \mathrm{U}_{3}(4), \mathrm{U}_{3}(5), \mathrm{U}_{3}(8), \mathrm{U}_{4}(2), \mathrm{U}_{4}(3), \mathrm{U}_{5}(2), \mathrm{U}_{5}(4), \mathrm{U}_{6}(2), \\
\mathrm{S}_{4}(4), \mathrm{S}_{6}(2), \mathrm{S}_{6}(3), \mathrm{S}_{8}(2), \mathrm{O}_{7}(3), \mathrm{O}_{8}^{+}(2), \mathrm{O}_{8}^{+}(3), \mathrm{O}_{8}^{-}(2), \mathrm{O}_{10}^{-}(2), \\
\left.{ }^{2} \mathrm{E}_{6}(2), \mathrm{F}_{4}(2),{ }^{2} \mathrm{~F}_{4}(2)^{\prime}, \mathrm{G}_{2}(3), \mathrm{G}_{2}(4),{ }^{3} \mathrm{D}_{4}(2)\right\}
\end{gathered}
$$

Proof. This follows immediately from Remark 4.1.3 and Corollaries 4.2.6, 4.3.4. $4.4 .2,4.5 .2,4.5 .4,4.6 .2$,

Remark 4.7.3. There is a strong resemblance between the statement of Theorem 4.7.1 and that of the Guralnick-Thompson conjecture, which was proven by D. Frohardt and K. Magaard, [15]. However, there does not appear to be a conceptual explanation for this resemblance. 


\section{5. $\ell$-Brauer $m$-Rational Groups}

Definition 5.0.4. Fix $\ell$ to be a prime and $m \geq 1$ an integer. Let $G$ be a finite group. We say that $G$ is $\ell$-Brauer $m$-rational if $[\mathbb{Q}(\phi): \mathbb{Q}] \mid m$ for all irreducible Brauer characters $\phi \in \operatorname{IBr}_{\ell}(G)$.

Note that rational groups are $\ell$-Brauer rational for every $\ell$. Every irreducible $\ell$ Brauer character, $\phi$ is a $\mathbb{Z}$-linear combination, $\phi=\sum a_{\chi} \chi^{\circ}$, where $\chi^{\circ}$ is the restriction

of the irreducible ordinary character $\chi \in \operatorname{Irr}(G)$ to the $\ell$-regular classes of $G$. As $\mathbb{Q}(\chi)=\mathbb{Q}$ for all $\chi$ in a rational group, $\mathbb{Q}(\phi)=\mathbb{Q}$ for all $\phi \in \operatorname{IBr}_{\ell}(G)$. It is not the case that an $m$-rational group is necessarily $\ell$-Brauer $m$-rational. To see this, consider $G=\mathrm{SL}_{2}(7)$ with $\ell=7$. Using the ordinary and modular character tables of $G$, we see that $G$ is quadratic rational, but is 7 -Brauer 4-rational. Of course, it is not the case that $\ell$-Brauer $m$-rational groups are $m$-rational. The alternating group $\mathrm{A}_{5}$ is 5-Brauer rational, but is not rational.

First, we state our main result for $\ell$-Brauer rational groups, i.e. $m=1$.

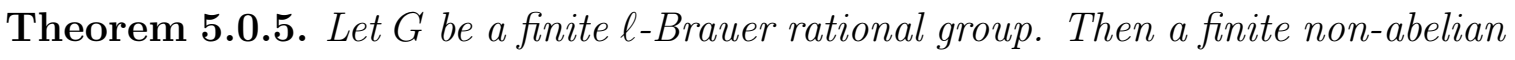
simple group $S$ can occur as a composition factor of $G$ if and only if $S$ is

- An alternating group $\mathrm{A}_{n}, n \geq 5$ for any prime $\ell$,

- One of the sporadic groups from the following set:

$$
\left\{\mathrm{M}_{12}, \mathrm{M}_{22}, \mathrm{~J}_{2}, \mathrm{Fi}_{22}, \mathrm{HS}, \mathrm{Suz}, \mathrm{HN}\right\} \text {, }
$$

- A group of Lie type in characteristic $\ell$, or

- One of the groups of Lie type in characteristic $p \neq \ell$ from the following set:

$$
\left\{\mathrm{L}_{2}(7), \mathrm{L}_{2}(8), \mathrm{L}_{2}(25), \mathrm{L}_{3}(2), \mathrm{L}_{3}(4), \mathrm{L}_{4}(3)\right.
$$




$$
\begin{gathered}
\mathrm{U}_{3}(3), \mathrm{U}_{3}(5), \mathrm{U}_{4}(2), \mathrm{U}_{4}(3), \mathrm{S}_{4}(4), \mathrm{S}_{6}(2), \\
\left.\mathrm{O}_{8}^{+}(2), \mathrm{O}_{8}^{+}(3), \mathrm{O}_{8}^{-}(2), \mathrm{G}_{2}(2)^{\prime},{ }^{3} \mathrm{D}_{4}(2)\right\}
\end{gathered}
$$

The proof of Theorem 5.0.5 uses the Classification of Finite Simple Groups, and the representation theory of finite groups of Lie type [6], [10]. Now we state our general version of the theorem for all natural numbers $m$.

Theorem 5.0.6. Let $G$ be a finite $\ell$-Brauer $m$-rational group. Then any non-abelian composition factor $S$ of $G$ is either an alternating group $\mathrm{A}_{n}, n \geq 5$, a sporadic group, a group of Lie type in characteristic $\ell$, or $S$ belongs to a finite set of groups of Lie type in characteristic $p \neq \ell, \mathcal{F}(m)$.

\subsection{Reduction To Simple Groups}

Throughout this chapter, $G$ is a (non-solvable) finite group, $\ell$ is a prime and $m \geq 1$ is an integer, $\operatorname{Irr}(G)$ is the set of irreducible complex characters of $G$, and $\operatorname{IBr}_{\ell}(G)$ is the set of irreducible $\ell$-Brauer characters of $G$. An irreducible Brauer character $\phi$ of $G$ is liftable to $\operatorname{Irr}(G)$ if there exists $\chi \in \operatorname{Irr}(G)$ such that $\phi=\chi^{\circ}$, the restriction of $\chi$ to the $\ell$-regular conjugacy classes of $G$. The following result gives a necessary character-theoretic condition for a non-abelian simple group $S$ to occur as a nonabelian composition factor of an $\ell$-Brauer $m$-rational group $G$.

Theorem 5.1.1. Suppose that $G$ is an $\ell$-Brauer $m$-rational group and $S$ is a nonabelian composition factor of $G$. Let $\alpha \in \operatorname{IBr}_{\ell}(S)$ be liftable to $\operatorname{Irr}(S)$. Then $[\mathbb{Q}(\alpha)$ : $\mathbb{Q}]|m| \operatorname{Out}(S) \mid$. In fact, there is a subgroup $R \leq \operatorname{Out}(S)$ such that the $R$-orbit of $\alpha$ has length $j$ and $[\mathbb{Q}(\alpha): \mathbb{Q}] \mid m j$.

To prove this reduction theorem, we first prove the following lemma. 
Lemma 5.1.2. Suppose that $G$ is an $\ell$-Brauer $m$-rational group with non-abelian minimal normal subgroup $N=S_{1} \times \cdots \times S_{n} \cong S^{n}$, where $S=S_{1}$. Then there exists a subgroup $R \leq \operatorname{Out}(S)$ such that for any $\alpha \in \operatorname{IBr}_{\ell}(S)$, the character

$$
\hat{\alpha}=\sum_{i=1}^{j} \alpha_{i}
$$

satisfies $[\mathbb{Q}(\hat{\alpha}): \mathbb{Q}] \mid m$, where $\left\{\alpha_{i} \mid 1 \leq i \leq j\right\}$ is the $R$-orbit of $\alpha$.

Proof. For any $g \in G$, conjugation gives an automorphism of $N$. As $\operatorname{Aut}(N)=$ $\operatorname{Aut}(S)$ 乙 $S_{n}$, we may consider those elements $g \in G$ that fix $S_{1}$, that is, the elements $g \in N_{G}\left(S_{1}\right)$. It is clear that we may naturally identify $N_{G}\left(S_{1}\right) /\left(S_{1} C_{G}\left(S_{1}\right)\right)$ with a subgroup $R \leq \operatorname{Out}(S)$.

Let $\alpha \in \operatorname{IBr}_{\ell}(S)$ be an irreducible $\ell$-Brauer character of $S$. Since the $R$-orbit of $1_{S}$ is the singleton set $\left\{1_{S}\right\}$, the result holds for the principal character, and thus we assume $\alpha \neq 1_{S}$. Consider the irreducible character $\beta=\alpha \otimes 1_{S_{2}} \otimes \cdots \otimes 1_{S_{n}} \in \operatorname{IBr}_{\ell}(N)$. Let $\phi \in \operatorname{IBr}_{\ell}(G)$ be an irreducible constituent of $\beta^{G}$. Restricting $\phi$ to $N$, we have $\phi_{N}=e \sum_{i=1}^{t} \beta^{g_{i}}$, where $\beta=\beta^{g_{1}}$, and $\left\{\beta^{g_{i}} \mid 1 \leq i \leq t\right\}$ is the $G$-orbit of $\beta$. Furthermore, $t=\left[G: \operatorname{Stab}_{G}(\beta)\right]$ and $\operatorname{Stab}_{G}(\beta) \leq N_{G}\left(S_{1}\right)$.

Now, we see that for any $g \in N_{G}\left(S_{1}\right)$ and $s \in S, \ell \nmid|s|$,

$$
\beta^{g}((s, 1, \ldots, 1))=\alpha^{g}(s) .
$$

That is, $\beta^{g}=\alpha^{g} \otimes 1_{S_{2}} \otimes \cdots \otimes 1_{S_{n}}$ for any $g \in N_{G}\left(S_{1}\right)$. Further, for $g \notin N_{G}\left(S_{1}\right)$, we have

$$
\beta^{g}(s, 1, \ldots, 1)=\beta\left(1, \ldots, 1, s^{\prime}, 1, \ldots, 1\right)=\alpha(1) \in \mathbb{Z} .
$$

Writing

$$
\phi_{N}=e\left(\sum_{i=1}^{j} \beta^{g_{i}}+\sum_{i=j+1}^{t} \beta^{g_{i}}\right),
$$


where $g_{1}, \ldots, g_{j} \in N_{G}\left(S_{1}\right)$ and $g_{j+1}, \ldots, g_{t} \notin N_{G}\left(S_{1}\right)$, we see that

$$
\begin{gathered}
\sum_{i=1}^{j} \beta^{g_{i}}((s, 1, \ldots, 1))=\frac{\phi_{N}((s, 1, \ldots, 1))}{e}-\sum_{i=j+1}^{t} \beta^{g_{i}}((s, 1, \ldots, 1)) \\
=\frac{\phi_{N}((s, 1, \ldots, 1))}{e}-(t-j) \alpha(1) \in \mathbb{Q}\left(\phi_{N}\right)
\end{gathered}
$$

for any $s \in S, \ell \nmid|s|$. However,

$$
\sum_{i=1}^{j} \beta^{g_{i}}((s, 1, \ldots, 1))=\sum_{i=1}^{j} \alpha^{g_{i}}(s)
$$

Now, we observe that $\left\{\alpha_{i}:=\alpha^{g_{i}} \mid 1 \leq i \leq j\right\}$ is the $R$-orbit of $\alpha$ since $\left\{\beta^{g_{i}} \mid 1 \leq i \leq j\right\}$ is the $N_{G}\left(S_{1}\right)$-orbit of $\beta$.

Finally, notice that $[\mathbb{Q}(\phi): \mathbb{Q}] \mid m$ since $G$ is $\ell$-Brauer $m$-rational, and thus $\left[\mathbb{Q}\left(\phi_{N}\right): \mathbb{Q}\right] \mid m$. Hence $[\mathbb{Q}(\hat{\alpha}): \mathbb{Q}] \mid m$ as needed.

We are now able to prove Theorem 5.1.1.

Proof. Suppose that $G$ is an $\ell$-Brauer $m$-rational group and $S$ is a non-abelian composition factor of $G$. As quotients of $\ell$-Brauer $m$-rational groups are again $\ell$ Brauer $m$-rational, we may assume that $N=S_{1} \times \cdots \times S_{n}$ is a minimal normal subgroup of $G$ with $S=S_{1}$. Let $\alpha \in \operatorname{IBr}_{\ell}(S)$ lift to $\chi \in \operatorname{Irr}(S)$, and define $R \leq \operatorname{Out}(S)$ and $\hat{\alpha}$ as in the previous lemma. Note that $\mathbb{Q}(\hat{\alpha}) \subseteq \mathbb{Q}(\alpha)$. Let $\Gamma=\operatorname{Gal}(\mathbb{Q}(\alpha) / \mathbb{Q}(\hat{\alpha}))$. Then for any $\tau \in \Gamma, \tau(\hat{\alpha})=\hat{\alpha}$, and we claim that $\tau$ permutes the constituents $\alpha_{i}$. Since $\chi^{\circ}=\alpha$, we have (in the notation of the previous proof) that $\alpha_{i}=\alpha^{g_{i}}=\left(\chi^{\circ}\right)^{g_{i}}=$ $\left(\chi^{g_{i}}\right)^{\circ}=: \chi_{i}^{\circ}$, and thus,

$$
\hat{\alpha}=\left(\sum_{i=1}^{j} \alpha_{i}\right)^{\tau}=\sum_{i=1}^{j}\left(\chi_{i}^{\tau}\right)^{\circ}
$$

Now, $\left(\chi^{\tau}\right)^{\circ}$ can be decomposed as $\beta_{1}+\cdots+\beta_{s}$ for some $\beta_{i} \in \operatorname{IBr}(S)$, and therefore, in the notation of the previous proof, we have

$$
\sum_{i=1}^{j}\left(\chi_{i}^{\tau}\right)^{\circ}=\left(\beta_{1}+\cdots+\beta_{s}\right)+\left(\beta_{1}+\cdots+\beta_{s}\right)^{g_{2}}+\cdots+\left(\beta_{1}+\cdots+\beta_{s}\right)^{g_{j}}
$$


By linear independence of the irreducible $\ell$-Brauer characters, it follows that $s=1$, and $\beta_{1}=\alpha_{1}^{g_{k}}$ for some $k$. That is, $\tau$ permutes the constituents of $\hat{\alpha}$. Finally, if $\tau$ fixes $\alpha$, then $\tau(\alpha(g))=\alpha(g)$ for all $g \in S$. Thus $\tau$ acts trivially on $\mathbb{Q}(\alpha)$, and must therefore be the identity element in $\Gamma$. As $\mathbb{Q}(\alpha)=\mathbb{Q}\left(\alpha_{i}\right)$ for all $1 \leq i \leq j$, $\operatorname{Stab}_{\Gamma}\left(\alpha_{i}\right)=1$ for all $1 \leq i \leq j$. By the orbit-stabilizer theorem, it follows that all $\Gamma$-orbits on $\left\{\alpha_{i} \mid 1 \leq i \leq j\right\}$ have length $|\Gamma|$. Therefore

$$
[\mathbb{Q}(\alpha): \mathbb{Q}(\hat{\alpha})]=|\Gamma||j||R||| \operatorname{Out}(S) \mid .
$$

Since $\hat{\alpha}$ satisfies $[\mathbb{Q}(\hat{\alpha}): \mathbb{Q}] \mid m$, we have $[\mathbb{Q}(\alpha): \mathbb{Q}]|m| \operatorname{Out}(S) \mid$, as desired.

Corollary 5.1.3. Suppose that $S$ is a non-abelian composition factor of an $\ell$-Brauer rational group. If $\operatorname{Out}(S)=1$, then $S$ is $\ell$-Brauer rational.

Proof. In the notation of Lemma 5.1.2, $R=1$, and hence $\hat{\alpha}=\alpha$ for any $\alpha \in \operatorname{IBr}_{\ell}(S)$. Therefore,

$$
[\mathbb{Q}(\hat{\alpha}): \mathbb{Q}]=[\mathbb{Q}(\alpha): \mathbb{Q}]=1 .
$$

That is, $S$ is $\ell$-Brauer rational.

The following fact will be helpful in dealing with simple non-abelian groups $S$ that are themselves $\ell$-Brauer rational.

Lemma 5.1.4. $S$ is $\ell$-Brauer rational if and only if $\chi(g) \in \mathbb{Z}$ for all $g \in S^{\circ}$ and $\chi \in \operatorname{Irr}(S)$.

Proof. Suppose $S$ is $\ell$-Brauer rational, and let $g \in S^{\circ}, \chi \in \operatorname{Irr}(S)$. Then

$$
\chi(g)=\chi^{\circ}(g)=\sum_{\phi \in \operatorname{IBr}_{\ell}(S)} d_{\chi, \phi} \phi,
$$

where $d_{\chi, \phi}$ is the (non-negative, integral) decomposition number, [37, Theorem 2.3, Corollary 2.9]. As each $\phi \in \operatorname{IBr}_{\ell}(S)$ is rational, it follows that $\chi(g)$ is rational. 
Conversely, suppose that $\chi(g) \in \mathbb{Q}$ for every $g \in S^{\circ}$ and $\chi \in \operatorname{Irr}(G)$, and let $\phi \in \operatorname{IBr}_{\ell}(S)$. Then, by Corollary 2.16 of [37], there exist $a_{\chi} \in \mathbb{Z}$ such that

$$
\phi=\sum_{\chi \in \operatorname{Irr}(S)} a_{\chi} \chi^{\circ} .
$$

Therefore, $\phi(g) \in \mathbb{Q}$ for all $g \in S^{\circ}$, and $S$ is $\ell$-Brauer rational.

Remark 5.1.5. We observe that the liftability condition in Theorem 5.1.1 is necessary by considering $S=A_{8}$, the alternating group on 8 letters. Using GAP, we see that the degree 4 character $\alpha \in \operatorname{IBr}_{2}(S)$ does not lift to an ordinary character of $S$ and has $\mathbb{Q}(\alpha)=\mathbb{Q}(\sqrt{-7}, \sqrt{-15})$. Therefore, $[\mathbb{Q}(\alpha): \mathbb{Q}]=4$. However, $A_{8}$ is a composition factor of $S_{8}$, a Brauer rational group.

Remark 5.1.6. Of course, the alternating groups occur as composition factors of the symmetric groups, which are rational, and hence $\ell$-Brauer $m$-rational for all primes $\ell$ and integers $m$.

In light of Theorem 5.1.1, we will attempt to construct irreducible $\ell$-Brauer characters of the finite simple groups of Lie type which take on highly irrational values. In particular, given a simple group of Lie type, $S$, we will try to find a liftable $\ell$-Brauer character $\alpha \in \operatorname{IBr}_{\ell}(S)$ satisfying $[\mathbb{Q}(\alpha): \mathbb{Q}]>m|\operatorname{Out}(S)|$. In order to construct these characters, we will again need primitive prime divisors, and thus we recall the definition.

Definition 5.1.7. Let $q$ and $n$ be integers larger than 1. A prime $r$ is a primitive prime divisor of $q^{n}-1$ if $r \mid q^{n}-1$, but $r \nmid q^{k}-1$ for all $1 \leq k<n$.

As we will see, the existence of these characters will also depend on the primes that divide the order of particular semisimple group elements, and thus, we will need the following result:

Lemma 5.1.8. Let $q \in \mathbb{Z}$ and $k, n \in \mathbb{N}$. Then $\left(q^{k}-1, q^{n}-1\right)=q^{(k, n)}-1$, where $(a, b)$ denotes the greatest common divisor of integers a and $b$. 
Proof. See, for example, [50, Lemma 5].

Finally, if $k$ is an integer, then we denote the 2-part of $k$ (i.e. the highest power of 2 dividing $k$ ) by $k_{2}$, and write $k_{2^{\prime}}:=\frac{k}{k_{2}}$, the $2^{\prime}$-part of $k$. In particular, if $a$ and $b$ are integers, then $(a, b)_{2^{\prime}}$ denotes the $2^{\prime}$-part of $(a, b)$. The following lemma will be useful in our computations:

Lemma 5.1.9. Suppose that $q$ is a natural number. Then for any natural number $n$,

$$
\begin{gathered}
\left(q^{n}+1\right)_{2}=\left\{\begin{array}{ll}
(q+1)_{2} & \text { if } n \text { is odd } \\
(2, q-1) & \text { if } n \text { is even }
\end{array},\right. \text { and } \\
\left(q^{n}-1\right)_{2}=\left\{\begin{array}{ll}
n_{2}(q+1)_{2} & \text { if } n \text { is even and } a \equiv-1 \bmod 4 \\
n_{2}(q-1)_{2} & \text { otherwise }
\end{array} .\right.
\end{gathered}
$$

Proof. See [50, Lemma 5 (i)].

\subsection{The Linear Groups}

Theorem 5.2.1. Let $S=\operatorname{PSL}_{n}(q)=\operatorname{PSL}_{n}\left(p^{f}\right), p \neq \ell, n \geq 4$ and $(n, q) \neq$ $(6,2),(7,2)$. Define $N=\frac{q^{n}-1}{(n, q-1)(q-1)}$ and $N^{\prime}=\frac{q^{n-1}-1}{(n, q-1)}$. Then, there exists a liftable irreducible character $\alpha \in \operatorname{IBr}_{\ell}(S)$ with either $[\mathbb{Q}(\alpha): \mathbb{Q}]=\frac{\phi(N)}{n}$ or $[\mathbb{Q}(\alpha): \mathbb{Q}]=\frac{\phi\left(N^{\prime}\right)}{n-1}$. Further, for a fixed $m \in \mathbb{N}$, only finitely many pairs $(n, q)$ satisfy $\frac{\phi(N)}{n}|m| \operatorname{Out}(S) \mid$ or $\frac{\phi\left(N^{\prime}\right)}{n-1}|m| \operatorname{Out}(S) \mid$. In general, $\operatorname{PSL}_{n}(q)$ can occur as a composition factor of an $\ell$-Brauer $m$-rational group for only finitely many pairs $(n, q)$.

As mentioned above, we will need primitive prime divisors to construct $\alpha$. Therefore, we first prove the result for $n=2$ and $n=3$, as a primitive prime divisor of $q^{2}-1$ does not necessarily exist, as well as for $\operatorname{PSL}_{6}(2)$ and $\operatorname{PSL}_{7}(2)$.

Lemma 5.2.2. Theorem 5.2.1 holds if $n=2$ and $n=3$. Further, $\mathrm{PSL}_{6}(2)$ and $\mathrm{PSL}_{7}(2)$ cannot occur as a composition factor of any $\ell$-Brauer rational group for any $\ell>2$. 
Proof. The character table of $\operatorname{PSL}_{2}(q)$ is known in CHEVIE, [19], and there exist irreducible characters $\alpha_{1}$ and $\alpha_{2}$ of degrees $q+1$ and $q-1$, respectively. Note that these are both semisimple characters corresponding to semisimple elements of coprime order (recall that $\mathrm{PSL}_{2}(2)$ and $\mathrm{PSL}_{2}(3)$ are not simple, and thus we assume $q>3$ ), and hence, at least one is an irreducible $\ell$-Brauer character when restricted to the $\ell$-regular classes. Further, they take on the values $\pm\left(\zeta_{1}+\zeta_{1}^{-1}\right)$, where $\zeta_{1}$ is a primitive $\frac{q^{2}-1}{(2, q-1)(q-1)}$-th root of unity and $\pm\left(\xi_{1}+\xi_{1}^{-1}\right)$, where $\xi_{1}$ is a primitive $\frac{q^{2-1}-1}{(2, q-1)}$-th root of unity. Thus, as both characters are rational on every other conjugacy class,

$$
\begin{gathered}
{\left[\mathbb{Q}\left(\alpha_{1}\right): \mathbb{Q}\right]=\frac{\phi\left(\frac{q^{2}-1}{(q-1)(2, q-1)}\right)}{2}, \quad \text { and }} \\
{\left[\mathbb{Q}\left(\alpha_{2}\right): \mathbb{Q}\right]=\frac{\phi\left(\frac{q^{2-1}-1}{(2, q-1)}\right)}{2} .}
\end{gathered}
$$

Likewise, the character table of $\operatorname{PSL}_{3}(q)$ is known in CHEVIE, [19], and there exist irreducible characters $\alpha_{1}$ and $\alpha_{2}$ of degrees $(q-1)^{2}(q+1)$ and $(q-1)\left(q^{2}+q+\right.$ 1), respectively. Note that these are both semisimple characters corresponding to semisimple elements of coprime order, and hence, at least one is an irreducible $\ell$ Brauer character when restricted to the $\ell$-regular classes. Further, they take on the values $\pm\left(\phi_{3}+\phi_{3}^{q}+\phi_{3}^{q^{2}}\right)$, where $\phi_{3}$ is a primitive $\frac{q^{3}-1}{(3, q-1)(q-1)}$-th root of unity and $\pm\left(\xi_{2}+\xi_{2}^{-1}\right)$, where $\xi_{2}$ is a primitive $\frac{q^{3-1}-1}{(3, q-1)}$-th root of unity. Thus,

$$
\begin{gathered}
\frac{\phi\left(\frac{q^{3}-1}{(q-1)(3, q-1)}\right)}{3} \mid\left[\mathbb{Q}\left(\alpha_{1}\right): \mathbb{Q}\right], \quad \text { and } \\
\frac{\phi\left(\frac{q^{3-1}-1}{(3, q-1)}\right)}{2} \mid\left[\mathbb{Q}\left(\alpha_{2}\right): \mathbb{Q}\right] .
\end{gathered}
$$

Now, we have that the odd prime divisors of $\left|\mathrm{PSL}_{6}(2)\right|$ are 3,5,7, and 31. Using GAP, we see that the 3,5, and 7 modular character tables have a liftable character $\alpha$ which takes on the value $\xi+\xi^{2}+\xi^{4}+\xi^{8}+\xi^{16}$ where $\xi$ is a primitive 31-st root of unity, and hence $6 \mid[\mathbb{Q}(\alpha): \mathbb{Q}]$, a violation of Theorem 5.1.1. Thus, $\operatorname{PSL}_{6}(2)$ cannot occur 
as a composition factor of any $\ell$-Brauer rational group for $\ell \in\{3,5,7\}$ or any prime $\ell$ not dividing $\left|\mathrm{PSL}_{6}(2)\right|$. Lastly, in GAP, we see that the 31-modular character table contains a liftable non-quadratic rational character, which again violates Theorem 5.1.1. Therefore, $\mathrm{PSL}_{6}(2)$ cannot occur as a composition factor of any $\ell$-Brauer rational group for any $\ell>2$.

The odd prime divisors of $\left|\mathrm{PSL}_{7}(2)\right|$ are 3,5,7,31, and 127. As before, the 2,5,7,31, and 127-modular character tables and the ordinary character table all have a liftable non-quadratic rational character, and therefore, by Theorem 5.1.1, $\mathrm{PSL}_{7}(2)$ cannot occur as a composition factor of any $\ell$-Brauer rational group for any $\ell>2$.

In view of Lemmas 3.1 .2 and 5.2 .2 , we now assume that $n \geq 4$ and $(n, q) \neq(6,2),(7,2)$; in particular, we may assume the existence of primitive prime divisors of $q^{n}-1$ and $q^{n-1}-1$. To prove Theorem 5.2.1 in the general case, we will need the following lemmas, which will generalize to the other groups of Lie type. We will work in the following setup: Let $\mathcal{G}$ be a simple algebraic group and $F$ a Frobenius endomorphism of $\mathcal{G}$ such that $\mathcal{G}^{F}=: L=\operatorname{SL}_{n}(q), S=L / Z(L)$, and $L^{*}=\operatorname{PGL}_{n}(q)$, the dual of $L$, see [6].

Lemma 5.2.3. Let $s$ be a generator of the unique subgroup of index $(n, q-1)$ in a cyclic maximal torus, $T^{*}$ of $L^{*}$ of order $\frac{q^{n}-1}{q-1}$. Then generators of $\langle s\rangle$ lie in $\frac{\phi(N)}{n}$ distinct $L^{*}$-conjugacy classes. Likewise, if $s^{\prime}$ is a generator of the unique subgroup of index $(n, q-1)$ in a cyclic maximal torus $T^{* \prime}$ of $L^{*}$ of order $q^{n-1}-1$, then the generators of $\left\langle s^{\prime}\right\rangle$ lie in $\frac{\phi\left(N^{\prime}\right)}{n-1}$ distinct $L^{*}$-conjugacy classes.

Proof. Choose $r$ to be a primitive prime divisor of $q^{n}-1$, and let $R^{*}$ be a Sylow $r$-subgroup of $L^{*}$. Note that $r|| s \mid$, and we may define $t=s^{|s| / r}$. Then $|t|=r$, and by Lemma 2.4 of [36], $t$ is regular semisimple, and hence, contained in a unique maximal torus of $L^{*}$, [10, Proposition 14.6(ii)]. As $R^{*}$ is the unique Sylow $r$-subgroup 
of $T^{*}$, it follows that $N_{L^{*}}\left(T^{*}\right) \leq N_{L^{*}}\left(R^{*}\right)$. Further, as $t$ is contained uniquely in $T^{*}$ and $\langle t\rangle=\Omega_{1}\left(R^{*}\right)$ (the subgroup of $R^{*}$ generated by all elements of order $r$ ), we have $N_{L^{*}}\left(R^{*}\right) \leq N_{L^{*}}\left(T^{*}\right)$, and hence equality. Now, suppose that $s^{i}$ and $s^{j}$ are generators of $\langle s\rangle$ that are $L^{*}$-conjugate, say $\left(s^{i}\right)^{h}=s^{j}$. Then

$$
\left(t^{i}\right)^{h}=\left(s^{|s| i / r}\right)^{h}=\left(\left(s^{i}\right)^{h}\right)^{|s| / r}=\left(s^{j}\right)^{|s| / r}=t^{j} .
$$

Hence, by Burnside's Fusion Control Lemma (note that $t^{i}, t^{j} \in Z\left(R^{*}\right)$ ), we have that $h \in N_{L^{*}}\left(R^{*}\right)=N_{L^{*}}\left(T^{*}\right)$. Observe that if $\left(s^{i}\right)^{h}=s^{j}$ for some $h \in N_{L^{*}}\left(T^{*}\right)$, then $\left(s^{i}\right)^{h a}=s^{j}$ for all $a \in T^{*}$, and thus, conjugacy classes are determined by $W\left(T^{*}\right)$ conjugacy. Since $\langle s\rangle$ is the unique subgroup of $T^{*}$ of order $|s|, N_{L^{*}}\left(T^{*}\right)$ acts on $\langle s\rangle$ and sends $s$ to another generator. Moreover, the stabilizer of a point under this action is $C_{L^{*}}(s)=T^{*}$, and thus the $\phi(N)$ generators of $\langle s\rangle$ lie in $L^{*}$-conjugacy classes of size $\left|W\left(T^{*}\right)\right|=n$, and thus, the generators of $\langle s\rangle$ lie in $\phi(N) / n$ distinct $L^{*}$-conjugacy classes.

Now choose $r^{\prime}$ to be a primitive prime divisor of $q^{n-1}-1$. Then, from Fermat's Little Theorem, we see that $n-1 \mid r^{\prime}-1$, and thus, either $r^{\prime}=n$ or $r^{\prime} \geq n+1$. First, we assume that $r^{\prime} \geq n+1$. Let $R^{* \prime}$ be a Sylow $r^{\prime}$-subgroup of $L^{*}$. Note that $r^{\prime}|| s^{\prime} \mid$, and we may define $t^{\prime}=s^{\prime\left|s^{\prime}\right| / r^{\prime}}$. Then $\left|t^{\prime}\right|=r^{\prime}$, and by Lemma 2.4 of [36], $t^{\prime}$ is regular semisimple, and hence, contained in a unique maximal torus of $L^{*},[10$, Proposition 14.6(ii)]. Further, by Lemma 13.14(i) and Remark 13.15(i) in [10], $t^{\prime}$ has connected centralizer. As $R^{* \prime}$ is the unique Sylow $r^{\prime}$-subgroup of $T^{* \prime}$, it follows that $N_{L^{*}}\left(T^{* \prime}\right) \leq N_{L^{*}}\left(R^{* \prime}\right)$. Further, as $t^{\prime}$ is contained uniquely in $T^{* \prime}$ and $\left\langle t^{\prime}\right\rangle=\Omega_{1}\left(R^{* \prime}\right)$, we have $N_{L^{*}}\left(R^{* \prime}\right) \leq N_{L^{*}}\left(T^{* \prime}\right)$, and hence equality. Now, suppose that $s^{i}$ and $s^{\prime j}$ are generators of $\left\langle s^{\prime}\right\rangle$ that are $L^{*}$-conjugate, say $\left(s^{\prime i}\right)^{h}=s^{\prime j}$. Then

$$
\left(t^{\prime i}\right)^{h}=\left(s^{\prime\left|s^{\prime}\right| i / r^{\prime}}\right)^{h}=\left(\left(s^{i}\right)^{h}\right)^{\left|s^{\prime}\right| / r^{\prime}}=\left(s^{\prime j}\right)^{\left|s^{\prime}\right| / r^{\prime}}=t^{\prime j} .
$$

Hence, by Burnside's Fusion Control Lemma (note that $t^{\prime i}, t^{\prime j} \in Z\left(R^{*}\right)$ ), we have that $h \in N_{L^{*}}\left(R^{*}\right)=N_{L^{*}}\left(T^{*}\right)$. Observe that if $\left(s^{\prime}\right)^{h}=s^{\prime j}$ for some $h \in N_{L^{*}}\left(T^{*}\right)$, then 
$\left(s^{i}\right)^{h a}=s^{\prime j}$ for all $a \in T^{*}$, and thus, conjugacy classes are determined by $W\left(T^{*}\right)$ conjugacy. Since $\left\langle s^{\prime}\right\rangle$ is the unique subgroup of $T^{* \prime}$ of order $\left|s^{\prime}\right|, N_{L^{*}}\left(T^{* \prime}\right)$ acts on $\left\langle s^{\prime}\right\rangle$ and sends $s^{\prime}$ to another generator. Moreover, the stabilizer of a point under this action is $C_{L^{*}}\left(s^{\prime}\right)=T^{* \prime}$, and thus the $\phi\left(N^{\prime}\right)$ generators of $\left\langle s^{\prime}\right\rangle$ lie in $L^{*}$-conjugacy classes of size $\left|W\left(T^{*}\right)\right|=n-1$, and thus, the generators of $\left\langle s^{\prime}\right\rangle$ lie in $\phi\left(N^{\prime}\right) /(n-1)$ distinct $L^{*}$-conjugacy classes.

Now, assume that $r^{\prime}=n$. Then the argument provided above fails, as the element of order $r^{\prime}, t^{\prime}$, may not have a connected centralizer. For example, consider $\mathcal{G}=\mathrm{SL}_{5}\left(\overline{\mathbb{F}}_{3}\right)$ and $\mathcal{G}^{*}=\mathrm{PGL}_{5}\left(\overline{\mathbb{F}}_{3}\right)$ so that $L=\mathrm{SL}_{5}(3)$ and $L^{*}=\operatorname{PGL}_{5}(3)$. Then a primitive prime divisor of $3^{5-1}-1$ is $5=n$. Let $\omega$ be a primitive 5 -th root of unity, and define the elements $g, h \in \mathcal{G}^{*}$ as follows:

$$
g=\left(\begin{array}{ccccc}
1 & 0 & 0 & 0 & 0 \\
0 & \omega & 0 & 0 & 0 \\
0 & 0 & \omega^{2} & 0 & 0 \\
0 & 0 & 0 & \omega^{3} & 0 \\
0 & 0 & 0 & 0 & \omega^{4}
\end{array}\right) \quad \text { and } \quad h=\left(\begin{array}{ccccc}
0 & 1 & 0 & 0 & 0 \\
0 & 0 & 1 & 0 & 0 \\
0 & 0 & 0 & 1 & 0 \\
0 & 0 & 0 & 0 & 1 \\
1 & 0 & 0 & 0 & 0
\end{array}\right)
$$

We have that $h g h^{-1}=\omega g$, and hence, $h \in C_{\mathcal{G}^{*}}(g)$. Thus $C_{\mathcal{G}^{*}}(g) \geq \mathcal{T}^{*}\langle h\rangle$, where $\mathcal{T}^{*}$ is a maximal torus containing $g$, and $g$ does not have a connected centralizer. However, returning to the general case, we may remedy this: as $n$ is a prime and $n=r^{\prime} \nmid q-1$, $Z(L)=1$. Hence, $L \cong L^{*} \cong S$, and we may switch the roles of $\mathcal{G}$ and $\mathcal{G}^{*}$. That is, now assume $\mathcal{G}=\mathrm{PGL}_{n}\left(\overline{\mathbb{F}}_{p}\right)$ and $\mathcal{G}^{*}=\mathrm{SL}_{n}\left(\overline{\mathbb{F}}_{p}\right)$. Then, choosing our element $t^{\prime}$ as before, $C_{\mathcal{G}^{*}}\left(t^{\prime}\right)$ is connected as $\mathcal{G}$ has connected center ([ $\underline{6}$, Theorem 4.5.9]), and the proof proceeds as in the case $r^{\prime} \geq n+1$.

Lemma 5.2.4. Let $s \in\left[L^{*}, L^{*}\right]$ be a semisimple regular $\ell^{\prime}$-element with connected centralizer. Then $\chi_{s}$, the semisimple character of L labeled by s is irreducible, restricts irreducibly to $S$, and $\chi_{s}^{\circ} \in \operatorname{IBr}_{\ell}(S)$. Further, if $\chi_{s}^{\circ}=\chi_{s^{k}}^{\circ}$, then $s$ is conjugate to $s^{k}$.

Proof. First, as $s$ is semisimple and regular with $C_{\mathcal{L}^{*}}(s)$ is connected, $\chi_{s} \in \operatorname{Irr}(L)$. Further, as $s \in\left[L^{*}, L^{*}\right], \operatorname{ker}\left(\chi_{s}\right) \geq Z(L)$, and hence, we may view $\chi_{s} \in \operatorname{Irr}(L / Z(L))$, 
that is, $\chi_{s} \in \operatorname{Irr}(S)$. Next, we observe that $\chi_{s}(1)=\left(\frac{|L|}{\left|C_{L^{*}}(s)\right|}\right)_{p^{\prime}}$, [6]. To prove that $\chi_{s}^{\circ} \in \operatorname{IBr}_{\ell}(S)$, we reference [24]. We have that $\chi_{s} \in \mathcal{E}(L, s)$, the Lusztig series labelled by $s$, and hence, $\chi_{s} \in \mathcal{E}_{\ell}(L, s):=\cup_{t} \mathcal{E}(L, s t)$, where $t$ runs through $\ell$-elements in $C_{\mathcal{L}^{*}}(s)$. By a result of Broué and Michel, $\mathcal{E}_{\ell}(L, s)$ is a union of $\ell$-blocks, and therefore, $\chi_{s}$ lies in some $\ell$-block, say $\operatorname{Irr}(B)$. By Proposition 2.3.3. $\left(\frac{|L|}{C_{L^{*}}(s)}\right)_{p^{\prime}} \mid \alpha(1)$ for any $\ell$-Brauer character in this block. As $\chi_{s}^{\circ}$ is a non-negative linear combination of the irreducible $\ell$-Brauer characters in $B$, it follows that $\chi_{s}^{\circ}$ is an irreducible $\ell$-Brauer character since $\chi_{s}(1)=\left(\frac{|L|}{\left|C_{L^{*}}(s)\right|}\right)_{p^{\prime}}$. Therefore $\chi_{s}^{\circ} \in \operatorname{IBr}_{\ell}(S)$.

Now, suppose that $\chi_{s}^{\circ}=\chi_{s^{k}}^{\circ}$. As above, $\mathcal{E}_{\ell}\left(L, s^{k}\right)$ is a union of $\ell$-blocks, say $B_{1} \cup \cdots \cup B_{n}$. By disjointedness of $\ell$-blocks and the assumption $\chi_{s}^{\circ}=\chi_{s^{k}}^{\circ}$, we may assume $B_{1}=B$, and therefore, $\mathcal{E}(L, s t) \cap \mathcal{E}\left(L, s^{k} u\right) \neq \emptyset$ for some $t, u, \ell$-elements. Now, Lusztig series' are disjoint, and hence, st must be conjugate to $s^{k} u$. As $s, s^{k}$ are $\ell^{\prime}$-elements, $u, t$ are $\ell$-elements, $s t=t s$, and $s^{k} u=u s^{k}$, we get that $s \sim s^{k}$.

We are now able to complete the proof of Theorem 5.2.1:

Proof. Notice from Lemma 5.1.8 that $\left(N, N^{\prime}\right)=1$, and in particular, either $s$ or $s^{\prime}$ as defined in Lemma 5.2 .3 is $\ell$-regular. Hence, in the notation of the previous lemma, either $\alpha:=\chi_{s}^{\circ}$ or $\alpha^{\prime}:=\chi_{s^{\prime}}^{\circ}$ is an irreducible $\ell$-Brauer character of $S$. As in the $m$-rational case, we see that the Galois automorphism sending $\theta$ to $\theta^{k}$, where $\theta$ is a primitive $N$-th (or $N^{\prime}$-th) root of unity acts trivially on $\mathbb{Q}(\alpha)\left(\right.$ or $\left.\mathbb{Q}\left(\alpha^{\prime}\right)\right) \Longleftrightarrow$ $\chi_{s}^{\circ}=\chi_{s^{k}}^{\circ}\left(\right.$ or $\left.\chi_{s^{\prime}}^{\circ}=\chi_{s^{k}}^{\circ}\right)$, by Lemma 4.2.4. Now, by the previous lemma, $\chi_{s}^{\circ}=\chi_{s^{k}}^{\circ}$ (or $\chi_{s^{\prime}}^{\circ}=\chi_{s^{\prime}}^{\circ}$ ) iff $s$ is conjugate to $s^{k}$ (or $s^{\prime}$ is conjugate to $s^{\prime k}$ ) $\Longleftrightarrow$ the maps sending $x$ to $x^{k}$ acts trivially on the conjugacy classes of generators of $\langle s\rangle$ (or $\left.\left\langle s^{\prime}\right\rangle\right)$. Note that the group consisting of the maps $x \mapsto x^{k}, k \in(\mathbb{Z} / N \mathbb{Z})^{\times}\left(\right.$or $\left.k \in\left(\mathbb{Z} / N^{\prime} \mathbb{Z}\right)^{\times}\right)$, acts transitively on the set of conjugacy classes of generators of $\langle s\rangle\left(\right.$ or $\left.\left\langle s^{\prime}\right\rangle\right)$. Thus, by the orbit stabilizer theorem, we have $[\mathbb{Q}(\alpha): \mathbb{Q}]=\frac{\phi(N)}{n}\left(\right.$ or $\left.\left[\mathbb{Q}\left(\alpha^{\prime}\right): \mathbb{Q}\right]=\frac{\phi\left(N^{\prime}\right)}{n-1}\right)$, since the generators of $\langle s\rangle$ (or $\left\langle s^{\prime}\right\rangle$ ) lie in $\frac{\phi(N)}{n}$ (or $\left.\frac{\phi\left(N^{\prime}\right)}{n-1}\right)$ conjugacy classes. Thus, we must determine when $\frac{\phi(N)}{n}>m|\operatorname{Out}(S)|$ and $\frac{\phi\left(N^{\prime}\right)}{n-1}>m|\operatorname{Out}(S)|$ to eliminate $S$ as a 
possible composition factor of an $\ell$-Brauer $m$-rational group.

Now, let $m \in \mathbb{N}$. We have from [29, Table 5.1.A] that

$$
|\operatorname{Out}(S)|=\left\{\begin{array}{ll}
(2, q-1) f & n=2 \\
2(n, q-1) f & n>2
\end{array} .\right.
$$

From Lemma 3.1.7, we see that

$$
\frac{\phi(N)}{n} \geq \sqrt{\frac{N}{2 n^{2}}} \geq \sqrt{\frac{q^{n-1}}{2 n^{3}}} \quad \text { and } \quad \frac{\phi\left(N^{\prime}\right)}{n-1} \geq \sqrt{\frac{N^{\prime}}{2(n-1)^{2}}} \geq \sqrt{\frac{q^{n-2}}{2 n^{2}}}
$$

Further, $m|\operatorname{Out}(S)| \leq 2 m(n, q-1) f \leq m q^{2}$. Comparing these, we must determine when

$$
\sqrt{\frac{q^{n-1}}{2 n^{3}}}>m q^{2} \quad \text { and } \quad \sqrt{\frac{q^{n-2}}{2 n^{2}}}>m q^{2} .
$$

Rewriting these as

$$
\sqrt{\frac{q^{n-5}}{2 n^{3}}} \geq \sqrt{\frac{2^{n-6}}{n^{3}}}>m \quad \text { and } \quad \sqrt{\frac{q^{n-6}}{2 n^{2}}} \geq \sqrt{\frac{2^{n-7}}{n^{2}}}>m,
$$

it is easy to see that there exists $n_{0} \in \mathbb{N}$ large enough so that $\frac{\phi(N)}{n}>m|\operatorname{Out}(S)|$ and $\frac{\phi\left(N^{\prime}\right)}{n-1}>m|\operatorname{Out}(S)|$ for all $n \geq n_{0}$ and $q \geq 2$. In particular, $\operatorname{PSL}_{n}(q)$ cannot occur as a composition factor of an $m$-rational group for any $n \geq n_{0}$ and $q \geq 2$. Now, for $4 \leq n \leq n_{0}$, we see that

$$
\frac{\phi(N)}{n} \geq \sqrt{\frac{N}{2 n^{2}}} \geq \sqrt{\frac{q^{n-1}}{2 n^{3}}} \quad \text { and } \quad \frac{\phi\left(N^{\prime}\right)}{n-1} \geq \sqrt{\frac{N^{\prime}}{2 n^{2}}} \geq \sqrt{\frac{q^{n-2}}{2 n^{2}}},
$$

and $2 m(n, q-1) f \leq 2 \log _{2}(q) n m$. As $\sqrt{\frac{q^{n-1}}{8 n^{5}\left(\log _{2}(q)\right)^{2}}}$ and $\sqrt{\frac{q^{n-2}}{8 n^{4}\left(\log _{2}(q)\right)^{2}}}$ are positive and increasing unboundedly for a fixed $4 \leq n \leq n_{0}$ and $q \geq 3$, there exists $q_{0}$ such that $\sqrt{\frac{q^{n-1}}{8 n^{5}\left(\log _{2}(q)\right)^{2}}}>m$ and $\sqrt{\frac{q^{n-2}}{8 n^{4}\left(\log _{2}(q)\right)^{2}}}>m$ for all $q \geq q_{0}$, and, in particular, $\frac{\phi(N)}{n}>2 m(n, q-1) f$ and $\frac{\phi\left(N^{\prime}\right)}{n-1}>2 m(n, q-1) f$ for all $q \geq q_{0}$. Therefore, only finitely many groups $\operatorname{PSL}_{n}(q)$ can occur as a composition factor of an $\ell$-Brauer $m$-rational group. 
Corollary 5.2.5. $\mathrm{PSL}_{n}(q)$ with $\ell \nmid q$ can occur as a composition factor of an $\ell$-Brauer rational group if and only if

$$
(n, q) \in\{(2,4),(2,5),(2,7),(2,8),(2,9),(2,25),(3,2),(3,4),(4,2),(4,3)\}
$$

To prove Corollary 5.2.5, we will need the following lemma, which is not specific to $\operatorname{PSL}_{n}(q)$, and will be used in the other sections.

Lemma 5.2.6. Suppose that $G$ is a finite group with a minimal normal subgroup $N=S_{1} \times \cdots \times S_{n} \cong S^{n}$. Let $\alpha \in \operatorname{IBr}_{\ell}(S)$, and define $\beta=\alpha \otimes 1_{S_{2}} \otimes \cdots \otimes 1_{S_{n}} \in \operatorname{IBr}_{\ell}(N)$. Let $\gamma \in \operatorname{IBr}_{\ell}\left(N_{G}\left(S_{1}\right) \mid \beta\right)$. Note that $N_{G}\left(S_{1}\right) \geq I$, the inertia subgroup of $\beta$ in $G$. Suppose further that any Galois-conjugate of $\gamma$ is an irreducible $\ell$-Brauer character of $N_{G}\left(S_{1}\right)$ and $\beta$ is rational. Then $\gamma$ is rational if and only if $\gamma^{G} \in \operatorname{IBr}_{\ell}(G)$ is rational. In particular, if $\beta$ is rational and $\gamma$ is liftable, then the conclusion holds.

Proof. Of course, if $\gamma$ is rational, then $\gamma^{G}$ is rational, by the formula for induced characters. Suppose that $\gamma^{G}$ is rational. Let $\sigma \in \operatorname{Gal}(\mathbb{Q}(\gamma) / \mathbb{Q})$. Notice that $\gamma^{\sigma} \in$ $\operatorname{IBr}\left(N_{G}\left(S_{1}\right) \mid \beta^{\sigma}\right)=\operatorname{IBr}\left(N_{G}\left(S_{1}\right) \mid \beta\right)$, as any Galois conjugate of $\gamma$ is an (irreducible) $\ell$-Brauer character of $N_{G}\left(S_{1}\right)$ and $\beta$ is rational. We see that

$$
\gamma^{G}=\left(\gamma^{G}\right)^{\sigma}=\left(\gamma^{\sigma}\right)^{G}
$$

As induction defines a bijection

$$
\operatorname{IBr}_{\ell}\left(N_{G}\left(S_{1}\right) \mid \beta\right) \rightarrow \operatorname{IBr}_{\ell}(G \mid \beta)
$$

and $\gamma^{G}=\left(\gamma^{\sigma}\right)^{G}$, it must be the case that $\gamma^{\sigma}=\gamma$. Therefore, $\gamma$ is rational.

We are now prepared to prove Corollary 5.2.5.

Proof. Fix $m=1$. From Theorem 5.2.1, we must determine when $\frac{\phi(N)}{n}|| \operatorname{Out}(S) \mid$ or $\frac{\phi\left(N^{\prime}\right)}{n-1}|| \operatorname{Out}(S) \mid$. In the notation of the proof of Theorem 5.2 .1 , we use a computer to determine that we may choose $n_{0}=19$. Then, for each $2 \leq n \leq 18$, we may choose $q_{0}$ as follows: 


\begin{tabular}{|c|c|c|c|c|c|c|c|c|c|c|c|c|c|c|c|c|c|}
\hline$n$ & 2 & 3 & 4 & 5 & 6 & 7 & 8 & 9 & 10 & 11 & 12 & 13 & 14 & 15 & 16 & 17 & 18 \\
\hline$q_{0}$ & 11684 & 377 & 390 & 56 & 22 & 12 & 9 & 7 & 6 & 5 & 5 & 4 & 4 & 4 & 4 & 3 & 3 \\
\hline
\end{tabular}

Verifying which of these (finitely many) pairs $(n, q)$ satisfy $\frac{\phi(N)}{n}|| \operatorname{Out}(S) \mid$ or $\frac{\phi\left(N^{\prime}\right)}{n-1}|| \operatorname{Out}(S) \mid$, we determine that $S=\operatorname{PSL}_{n}(q)$ can occur as a composition factor of an $\ell$-Brauer rational group only if

$$
\begin{gathered}
(n, q) \in\{(2,4),(2,5),(2,7),(2,8),(2,9),(2,11),(2,13),(2,19),(2,23),(2,25),(2,27), \\
(3,2),(3,3),(3,4),(3,7),(3,16),(4,2),(4,3),(5,2)\} .
\end{gathered}
$$

As $\mathrm{L}_{2}(4) \cong \mathrm{L}_{2}(5) \cong \mathrm{A}_{5}, \mathrm{~L}_{2}(9) \cong \mathrm{A}_{6}$, and $\mathrm{L}_{4}(2) \cong \mathrm{A}_{8}$, we have that these occur as composition factors of $\ell$-Brauer rational groups, namely $S_{5}, S_{6}$, and $S_{8}$, for any prime $\ell$.

We have that the (non-defining characteristic) prime divisors of $\left|\mathrm{L}_{2}(7)\right|$ are 2 and 3. We see that $L_{2}(7) .2$ is 2 -Brauer rational. To show that $L_{2}(7)$ cannot occur as a composition factor of any 3-Brauer rational group, we invoke Lemma 5.2.6. Suppose that $G$ is 3-Brauer rational, with minimal normal subgroup $N=S_{1} \times \cdots \times S_{n}$, $S_{i} \cong \mathrm{L}_{2}(7)=: S$. We have that $N_{G}\left(S_{1}\right) / C_{G}\left(S_{1}\right) \cong S$ or $N_{G}\left(S_{1}\right) / C_{G}\left(S_{1}\right) \cong S .2$. In the former case, we see that $S$, and hence $N_{G}\left(S_{1}\right)$ have an irrational 3-Brauer character $\chi$ of degree 3 . Using the formula for the induced character, it is clear to see that $\chi^{G} \in \operatorname{IBr}_{3}(G)$ is not a rational character by restricting to $S_{1}$. In the latter case, we see that $S$ has a rational character $\chi \in \operatorname{IBr}_{3}(S)$ that extends to an irrational irreducible character $\psi \in \operatorname{IBr}_{3}(S .2)$. Thus, there exists an irrational irreducible character, the inflation $\hat{\psi}$, of $N_{G}\left(S_{1}\right)$ lying above $\chi \otimes 1_{S_{2}} \otimes \cdots \otimes 1_{S_{n}}$. Further, we see that the Galois conjugates of $\hat{\psi}$ are irreducible 3 -Brauer characters (in fact, $\hat{\psi}$ is liftable), and therefore, by Lemma 5.2.6, $\hat{\psi}^{G}$ is an irrational irreducible 3-Brauer character of $G$. Therefore, $\mathrm{L}_{2}(7)$ cannot occur as a composition factor of any 3-Brauer rational group. The same irreducible characters and the same argument also prove that $\mathrm{L}_{2}(7)$ cannot occur as a composition factor of any $\ell$-Brauer rational group for $\ell$ not dividing $\left|L_{2}(7)\right|$. 
We have that the (non-defining characteristic) prime divisors of $\left|\mathrm{L}_{2}(8)\right|$ are 3 and 7. Using GAP, we see that $\mathrm{L}_{2}(8) .3$ is a 3 -Brauer rational group. To show that $\mathrm{L}_{2}(8)$ cannot occur as a composition factor of any 7-Brauer rational group, mimic the proof of the proof for $\mathrm{L}_{2}(7)$. In the case that $N_{G}\left(S_{1}\right) / C_{G}\left(S_{1}\right) \cong S$, use an irrational character of degree 7 . In the case that $N_{G}\left(S_{1}\right) / C_{G}\left(S_{1}\right) \cong S .3$, use a rational character of degree 8 that extends to an irrational character of S.3. The same characters and arguments also prove that $\mathrm{L}_{2}(8)$ cannot occur as a composition factor of any $\ell$-Brauer rational group with $\ell$ not dividing $\left|\mathrm{L}_{2}(8)\right|$.

We have that the (non-defining characteristic) prime divisors of $\left|\mathrm{L}_{2}(11)\right|$ are 2, 3 and 5. To show that $\mathrm{L}_{2}(11)$ cannot occur as a composition factor of any 2-Brauer or 3 -Brauer rational group, we observe that $\mathrm{L}_{2}(11)$ has an irrational liftable irreducible character which extends to $\mathrm{L}_{2}(11) .2$, a clear violation of Lemma 5.1.2. To show that $\mathrm{L}_{2}(11)$ cannot occur as a composition factor of any 5 -Brauer or $\ell$-Brauer rational group, where $\ell \nmid\left|\mathrm{L}_{2}(11)\right|$, apply the same argument as in $\mathrm{L}_{2}(7)$. In the case that $N_{G}\left(S_{1}\right) / C_{G}\left(S_{1}\right) \cong S$, use an irreducible character of degree 5 , and in the case that $N_{G}\left(S_{1}\right) / C_{G}\left(S_{1}\right) \cong S .2$, use a rational character of $S$ of degree 10 that extends to an irrational liftable character of $S .2$.

We have that the (non-defining characteristic) prime divisors of $\left|\mathrm{L}_{2}(13)\right|$ are 2, 3 and 7 . To show that $\mathrm{L}_{2}(13)$ cannot occur as a composition factor of any 2-Brauer or 3-Brauer rational group, we observe that $\mathrm{L}_{2}(13)$ has an irrational liftable irreducible character which extends to $\mathrm{L}_{2}(13) .2$, a clear violation of Lemma 5.1.2. To show that $\mathrm{L}_{2}(13)$ cannot occur as a composition factor of any 7 -Brauer or $\ell$-Brauer rational group, where $\ell \nmid\left|\mathrm{L}_{2}(13)\right|$, apply the same argument as in $\mathrm{L}_{2}(7)$. In the case that $N_{G}\left(S_{1}\right) / C_{G}\left(S_{1}\right) \cong S$, use an irreducible character of degree 7 , and in the case that $N_{G}\left(S_{1}\right) / C_{G}\left(S_{1}\right) \cong S .2$, use a rational character of $S$ of degree 14 that extends to an irrational liftable character of $S .2$.

We have that the (non-defining characteristic) prime divisors of $\left|\mathrm{L}_{2}(19)\right|$ are 2, 3 and 5. To show that $\mathrm{L}_{2}(19)$ cannot occur as a composition factor of any 2-Brauer, 
3-Brauer, 5-Brauer, or $\ell$-Brauer rational group with $\ell \nmid\left|\mathrm{L}_{2}(19)\right|$, we observe that $\mathrm{L}_{2}(19)$ has an irrational liftable irreducible character which extends to $\mathrm{L}_{2}(19) .2$, a clear violation of Lemma 5.1.2.

We have that the (non-defining characteristic) prime divisors of $\left|\mathrm{L}_{2}(23)\right|$ are 2,3 and 11. To show that $\mathrm{L}_{2}(13)$ cannot occur as a composition factor of any 2-Brauer, 3 -Brauer or $\ell$-Brauer rational group, where $\ell \nmid\left|\mathrm{L}_{2}(23)\right|$, we observe that $\mathrm{L}_{2}(23)$ has an irrational liftable irreducible character which extends to $\mathrm{L}_{2}(23) .2$, a clear violation of Lemma 5.1.2. To show that $\mathrm{L}_{2}(23)$ cannot occur as a composition factor of any 11-Brauer rational group, apply the same argument as in $\mathrm{L}_{2}(7)$. In the case that $N_{G}\left(S_{1}\right) / C_{G}\left(S_{1}\right) \cong S$, use an irreducible character of degree 11, and in the case that $N_{G}\left(S_{1}\right) / C_{G}\left(S_{1}\right) \cong S .2$, use a rational character of $S$ of degree 22 that extends to an irrational liftable character of S.2.

We have that the (non-defining characteristic) prime divisors of $\left|\mathrm{L}_{2}(25)\right|$ are 2,3 and 13. To show that $\mathrm{L}_{2}(25)$ cannot occur as a composition factor of any 2-Brauer, 3 -Brauer or $\ell$-Brauer rational group, where $\ell \nmid\left|\mathrm{L}_{2}(25)\right|$, we observe that $\mathrm{L}_{2}(25)$ has a liftable irreducible character $\chi$ which satisfies $6 \mid[\mathbb{Q}(\chi): \mathbb{Q}]$, a clear violation of Theorem 5.1.1, as $\left|\operatorname{Out}\left(\mathrm{L}_{2}(25)\right)\right|=4$. Further, using GAP, we see that $\mathrm{L}_{2}(25) .2_{2}$ is 13-Brauer rational.

We have that the (non-defining characteristic) prime divisors of $\left|\mathrm{L}_{2}(27)\right|$ are 2,7 and 13. To show that $\mathrm{L}_{2}(27)$ cannot occur as a composition factor of any 2-Brauer, 7-Brauer, 13-Brauer, or $\ell$-Brauer rational group, where $\ell \nmid\left|\mathrm{L}_{2}(27)\right|$, we observe that $\mathrm{L}_{2}(27)$ has a liftable irreducible character $\chi$ which satisfies $6 \mid[\mathbb{Q}(\chi): \mathbb{Q}]$ and extends to $\mathrm{L}_{2}(27) .2$, a clear violation of Lemma 5.1 .2 as $\left|\operatorname{Out}\left(\mathrm{L}_{2}(27)\right)\right|=6$.

We have that $\mathrm{L}_{3}(2) \cong \mathrm{L}_{2}(7)$, and thus, from our work above with $\mathrm{L}_{2}(7)$, we must only check our result for the prime 7 . Using GAP, we see that $\mathrm{L}_{3}(2)$ is in fact 7 -Brauer rational.

We have that the (non-defining characteristic) prime divisors of $\left|\mathrm{L}_{3}(3)\right|$ are 2 and 13. To show that $\mathrm{L}_{3}(3)$ cannot occur as a composition factor of any 2-Brauer 
or $\ell$-Brauer rational group, where $\ell \nmid\left|\mathrm{L}_{3}(3)\right|$, we observe that $\mathrm{L}_{3}(3)$ has a liftable irreducible character $\chi$ which satisfies $4 \mid[\mathbb{Q}(\chi): \mathbb{Q}]$, a clear violation of Theorem 5.1.1, as $\left|\operatorname{Out}\left(\mathrm{L}_{3}(3)\right)\right|=2$. In the case that $\ell=13$, however, we observe that Lemma 5.2.6 does not apply: the Galois conjugates of the candidate characters are NOT Brauer characters. Thus, we prove the following lemma which will be necessary for eliminating $\mathrm{L}_{3}(3)$ and six sporadic groups as possible composition factors of $\ell$-Brauer rational groups.

Lemma 5.2.7. Suppose that $S$ is a finite nonabelian simple group and $\operatorname{Aut}(S)=S .2$. Assume that there exists $1_{S} \neq \alpha \in \operatorname{IBr}_{\ell}(S)$ that extends to an irreducible character $\tilde{\alpha} \in \operatorname{IBr}_{\ell}(S .2)$ and $S$ has an irrational character $\alpha^{\prime} \neq \alpha \in \operatorname{IBr}_{\ell}(S)$. If $S$ occurs as a composition factor of some $\ell$-Brauer rational group, then $\alpha$ is rational and $\operatorname{IBr}_{\ell}(S .2 \mid \alpha)$ is rational.

Proof. Assume $G$ is an $\ell$-Brauer rational group with minimal normal subgroup $N=$ $S_{1} \times \cdots \times S_{n} \cong S^{n}$. Then $N_{G}\left(S_{1}\right) / C_{G}\left(S_{1}\right) \cong S$ or $N_{G}\left(S_{1}\right) / C_{G}\left(S_{1}\right) \cong S .2$. In the first case, we may inflate $\alpha^{\prime}$ to an irrational character of $N_{G}\left(S_{1}\right)$ lying above $\beta^{\prime}=\alpha^{\prime} \otimes 1_{S_{2}} \otimes \cdots \otimes 1_{S_{n}}$. Using the formula for induced characters, we see that this inflated character induced to $G$ is irrational. Thus, we must be in the case that $N_{G}\left(S_{1}\right) / C_{G}\left(S_{1}\right) \cong S .2$. If $\alpha$ is irrational and extends to $S .2$, then we contradict Lemma 5.1.2. We claim that $\operatorname{IBr}_{\ell}(S .2 \mid \alpha)$ is rational. Suppose by way of contradiction that there exists $x \in S .2 \backslash S$ such that $\tilde{\alpha}(x) \notin \mathbb{Q}$. Let $g \in N_{G}\left(S_{1}\right)$ be a lift of $x$. Take $\gamma$ to be the inflation of $\tilde{\alpha}$ to $N_{G}\left(S_{1}\right)$. Then $\gamma$ lies above $\beta=\alpha \otimes 1_{S_{2}} \otimes \cdots \otimes 1_{S_{n}}$. Define $\chi:=\gamma^{G} \in \operatorname{IBr}_{\ell}(G \mid \beta)$. Let $V$ be the module affording $\chi$, and decompose $V_{N_{G}\left(S_{1}\right)}=V_{1} \oplus \cdots \oplus V_{n}$, where $V_{1}$ affords $\gamma$. We claim that $\chi(g) \notin \mathbb{Q}$. As $g \in N_{G}\left(S_{1}\right)$, $g$ fixes $V_{1}$, and say without loss of generality that $g$ also fixes $V_{2}, \ldots, V_{j}$ and has no fixed points on $V_{j+1}, \ldots, V_{n}$. Then $\chi_{\langle g\rangle}=\chi_{1}+\cdots+\chi_{j}+\delta$, where $\chi_{i}$ is the character afforded by $V_{i}$ and $\delta$ is afforded by $V_{j+1} \oplus \cdots \oplus V_{n}$. As $\gamma$ is the inflation of $\tilde{\alpha}$ and corresponds to $V_{1}$, we have $\chi_{1}(g)=\tilde{\alpha}(x) \notin \mathbb{Q}$. 
Now, suppose that $y \in G$ acts on $S_{1}$ by $S_{1}^{y}=S_{i}$ for some $1 \leq i \leq j$. Then we see

$$
g \in \operatorname{Stab}_{G}\left(V_{i}\right)=\operatorname{Stab}_{G}\left(V_{1}\right)^{y}=N_{G}\left(S_{1}\right)^{y}=N_{G}\left(S_{i}\right),
$$

and thus, $g$ acts as an automorphism of $S_{i}$. If $g$ acts as an inner automorphism, then, of course, $\chi_{i}(g) \in \mathbb{Q}$. However, if $g$ acts as an outer automorphism, there exists $z_{i} \in S_{i}$ such that $g z_{i}=x$ as $\operatorname{Aut}(S)=S .2$. Note that multiplication by $z_{i}$ does not affect the action of $g$ on any $S_{d}$ with $d \neq i$. Then

$$
\chi_{i}\left(g z_{i}\right)=\chi_{i}(x)=\tilde{\alpha}(x)
$$

Lastly, we claim that $\delta(g)=0$. Assuming this is the case, we see that

$$
\chi(g)=\chi_{1}(g)+\cdots+\chi_{j}(g)+\delta(g)=k \tilde{\alpha}(x)+k^{\prime},
$$

for some $k, k^{\prime} \in \mathbb{Z}, k>0$, a contradiction, as $\chi(g) \in \mathbb{Q}$ and $\tilde{\alpha}(x) \notin \mathbb{Q}$. Thus, the proof is complete once we prove the following lemma.

Lemma 5.2.8. Suppose $\langle g\rangle$ acts on $V=V_{1} \oplus \cdots \oplus V_{m}$ by $g: V_{1} \mapsto V_{2} \mapsto \ldots \mapsto V_{m} \mapsto$ $V_{1}$ and $|g|$ is coprime to $\operatorname{char}(\mathbb{F})$. Then $\delta(g)=0$, where $\delta$ is the $\ell$-Brauer character afforded by $V$.

Proof. First, we notice that $g^{m}$ fixes each of $V_{1}, \ldots, V_{m}$. Thus, we may diagonalize $g^{m}$ on $V_{1}$, say with basis $V_{1}=\left\langle e_{1}, \ldots, e_{n}\right\rangle$. That is, $g^{m}\left(e_{i}\right)=\lambda_{i} e_{i}$ for some scalars $\lambda_{i}$. For each $\lambda_{i}$, we have distinct $\mu_{i 1}, \ldots, \mu_{i m}$ such that $\mu_{i j}^{m}=\lambda_{i}$. For each $1 \leq j \leq m$, define

$$
e_{i j}=e_{i}+\mu_{i j}^{-1} g\left(e_{i}\right)+\mu_{i j}^{-2} g^{2}\left(e_{i}\right)+\cdots+\mu_{i j}^{1-m} g^{m-1}\left(e_{i}\right) \in V .
$$

Then, we see that

$$
\begin{gathered}
g\left(e_{i j}\right)=g\left(e_{i}\right)+\mu_{i j}^{-1} g^{2}\left(e_{i}\right)+\mu_{i j}^{-2} g^{3}\left(e_{i}\right)+\cdots+\mu_{i j}^{1-m} g^{m}\left(e_{i}\right) \\
=\mu_{i j}\left(\mu_{i j}^{-m} g^{m}\left(e_{i}\right)+\mu_{i j}^{-1} g\left(e_{i}\right)+\mu_{i j}^{-2} g^{2}\left(e_{i}\right)+\mu_{i j}^{-3} g^{3}\left(e_{i}\right)+\cdots+\mu_{i j}^{1-m} g^{m-1}\left(e_{i}\right)\right)
\end{gathered}
$$




$$
=\mu_{i j}\left(\lambda^{-1} \lambda e_{i}+\mu_{i j}^{-1} g\left(e_{i}\right)+\mu_{i j}^{-2} g^{2}\left(e_{i}\right)+\mu_{i j}^{-3} g^{3}\left(e_{i}\right)+\cdots+\mu_{i j}^{1-m} g^{m-1}\left(e_{i}\right)\right)=\mu_{i j} e_{i j} .
$$

Thus, $g$ has $n \cdot m$ distinct eigenvalues, and so $\delta(g)=\sum \tilde{\mu}_{i j}$, where $\tilde{\mu}_{i j}$ are lifts of $\mu_{i j}$ to complex numbers. However,

$$
\sum \tilde{\mu}_{i j}=\sum_{i} \sum_{j} \tilde{\mu}_{i j}=\sum_{i}\left(\sum_{j} \tilde{\epsilon}^{j-1}\right) \tilde{\mu}_{i 1},
$$

where $\epsilon$ is a primitive $m$-th root of unity. As $\sum_{j} \tilde{\epsilon}^{j-1}=0$, we have that $\delta(g)=0$.

Using GAP, we see that (in the notation of Lemma 5.2.7) we may choose $\alpha^{\prime}$ to be the irrational character of degree 26 in $S$ and $\alpha$ to be a rational character of degree 16 that extends to an irrational character of $S .2$. Since $\operatorname{Aut}\left(\mathrm{L}_{3}(3)\right)=S .2, \mathrm{~L}_{3}(3)$ cannot occur as a composition factor of any 13-Brauer rational group, by the Lemma 5.2.7.

Using GAP, we see that the ordinary character table of $\operatorname{Aut}\left(\mathrm{L}_{3}(4)\right)$ is rational, and therefore, $\mathrm{L}_{3}(4)$ occurs as a composition factor of some $\ell$-Brauer rational group for any $\ell$.

We have that the (non-defining characteristic) prime divisors of $\left|\mathrm{L}_{3}(7)\right|$ are 2,3 , and 19. To show that $\mathrm{L}_{3}(7)$ cannot occur as a composition factor of any 2-Brauer, 3-Brauer, or $\ell$-Brauer rational group, where $\ell \nmid\left|\mathrm{L}_{3}(3)\right|$, we observe that $\mathrm{L}_{3}(7)$ has a liftable irreducible character $\chi$ which satisfies $6 \mid[\mathbb{Q}(\chi): \mathbb{Q}]$ and extends to $\mathrm{L}_{3}(7) .3$, a clear violation of Lemma 5.1 .2 as $\left|\operatorname{Out}\left(\mathrm{L}_{3}(7)\right)\right|=6$. To show that $\mathrm{L}_{3}(7)$ cannot occur as a composition factor of any 19-Brauer rational group, we observe that $\mathrm{L}_{3}(7)$ has a liftable irreducible character $\chi$ which satisfies $4 \mid[\mathbb{Q}(\chi): \mathbb{Q}]$, a clear violation of Theorem 5.1.1 as $\left|\operatorname{Out}\left(\mathrm{L}_{3}(7)\right)\right|=6$.

We have that the (non-defining characteristic) prime divisors of $\left|\mathrm{L}_{3}(16)\right|$ are 3 , $5,7,13$, and 17 . We see that $\frac{16^{3-1}-1}{(3,16-1)}=85=5 \cdot 17$. Thus, the irreducible character $\chi$ corresponding to the semisimple element in $\mathrm{PGL}_{3}(16)$ of order 85 may be viewed as an irreducible 3-Brauer, 7-Brauer, 13-Brauer, or $\ell$-Brauer character of $\mathrm{L}_{3}(16)$, where $\ell \nmid\left|\mathrm{L}_{3}(16)\right|$. However, we see that $32 \mid[\mathbb{Q}(\chi): \mathbb{Q}]$ by Lemma 5.2.2, a clear violation of Theorem 5.1.1, as $\left|\operatorname{Out}\left(\mathrm{L}_{3}(16)\right)\right|=24$. Hence, $\mathrm{L}_{3}(16)$ 
cannot occur as a composition factor of any 3-Brauer, 7-Brauer, 13-Brauer, or $\ell$ Brauer rational group, where $\ell \nmid\left|\mathrm{L}_{3}(16)\right|$. To show that $\mathrm{L}_{3}(16)$ cannot occur as a composition factor of any 5-Brauer or 17-Brauer rational group, we notice that the outer diagonal automorphisms form a cyclic group of order 3. As these act trivially on the irreducible $\ell$-Brauer characters coming from semisimple characters, we have that (in the notation of Lemma 5.1.2) the $R$-orbit length of any such $\alpha$ has length at most 8. However, using an element of order 91 in $\mathrm{SL}_{3}(16)$ yields a character $\alpha$ satisfying $24 \mid[\mathbb{Q}(\alpha)$ : $\mathbb{Q}]$ by Lemma 5.2.2. Hence, by Theorem 5.1.1, $\mathrm{L}_{3}(16)$ cannot occur as a composition factor of any 5-Brauer or 17-Brauer rational group.

We have that the (non-defining characteristic) prime divisors of $\left|\mathrm{L}_{4}(3)\right|$ are 2,5 , and 13. Using GAP, we see that $\mathrm{L}_{4}(3) .2_{2}$ is 13 -Brauer rational. To show that $\mathrm{L}_{4}(3)$ cannot occur as a composition factor of any 2-Brauer, 5-Brauer, or $\ell$-Brauer rational group, where $\ell \nmid\left|\mathrm{L}_{4}(3)\right|$, we observe that $\mathrm{L}_{4}(3)$ has a liftable irreducible character $\chi$ which satisfies $4 \mid[\mathbb{Q}(\chi): \mathbb{Q}]$ and extends to $\mathrm{L}_{4}(3) .2_{1}$, a clear violation of Lemma 5.1 .2 as $\left|\operatorname{Out}\left(\mathrm{L}_{4}(3)\right)\right|=4$.

We have that the (non-defining characteristic) prime divisors of $\left|\mathrm{L}_{5}(2)\right|$ are 3,5 , 7, and 31. To show that $\mathrm{L}_{5}(2)$ cannot occur as a composition factor of any 3-Brauer, 5-Brauer, 7-Brauer or $\ell$-Brauer rational group, where $\ell \nmid\left|\mathrm{L}_{5}(2)\right|$, we observe that $\mathrm{L}_{5}(2)$ has a liftable irreducible character $\chi$ which satisfies $6 \mid[\mathbb{Q}(\chi): \mathbb{Q}]$, a clear violation of Theorem 5.1.1, as $\left|\operatorname{Out}\left(\mathrm{L}_{5}(2)\right)\right|=2$. To show that $\mathrm{L}_{5}(2)$ cannot occur as a composition factor of any 31-Brauer rational group, apply the same argument as in $\mathrm{L}_{2}(7)$. In the case that $N_{G}\left(S_{1}\right) / C_{G}\left(S_{1}\right) \cong S$, use an irreducible character of degree 465 , and in the case that $N_{G}\left(S_{1}\right) / C_{G}\left(S_{1}\right) \cong S .2$, use a rational character of $S$ of degree 930 that extends to an irrational liftable character of S.2.

\begin{tabular}{|c|c|c|c|}
\hline$(n, q)$ & $(2,4)$ & $(2,5)$ & $(2,7)$ \\
\hline Prime(s) $\ell$ & Any prime $\ell$ & Any Prime $\ell$ & 2 \\
\hline$\ell$-Brauer Rational Group & $\mathrm{L}_{2}(4) .2$ & $\mathrm{~L}_{2}(5) .2$ & $\mathrm{~L}_{2}(7) .2$ \\
\hline
\end{tabular}




\begin{tabular}{|c|c|c|c|}
\hline$(n, q)$ & $(2,8)$ & $(2,9)$ & $(2,25)$ \\
\hline Prime $(\mathrm{s}) \ell$ & 3 & Any prime $\ell$ & 13 \\
\hline Ł-Brauer Rational Group & $\mathrm{L}_{2}(8) .3$ & $\mathrm{~L}_{2}(9) .2$ & $\mathrm{~L}_{2}(25) .2_{2}$ \\
\hline
\end{tabular}

\begin{tabular}{|c|c|c|c|c|}
\hline$(n, q)$ & $(3,2)$ & $(3,4)$ & $(4,2)$ & $(4,3)$ \\
\hline Prime $(\mathrm{s}) \ell$ & 7 & Any Prime $\ell$ & Any prime $\ell$ & 13 \\
\hline$\ell$-Brauer Rational Group & $\mathrm{L}_{3}(2)$ & Aut $\left(\mathrm{L}_{3}(4)\right)$ & $\mathrm{L}_{4}(2) .2$ & $\mathrm{~L}_{4}(3) .2_{2}$ \\
\hline
\end{tabular}

Note that $\mathrm{L}_{2}(4) \cong \mathrm{L}_{2}(5) \cong \mathrm{A}_{5}, \mathrm{~L}_{2}(9) \cong \mathrm{A}_{6}$, and $\mathrm{L}_{4}(2) \cong \mathrm{A}_{8}$, and thus, we remove $\mathrm{L}_{2}(4), \mathrm{L}_{2}(5), \mathrm{L}_{2}(9)$, and $\mathrm{L}_{4}(2)$ from our list in the statement of Theorem 5.0.5.

\subsection{The Unitary Groups}

Theorem 5.3.1. Let $S=\operatorname{PSU}_{n}(q), n \geq 3, q^{2}=p^{f}$ and $p \neq \ell$. Then $S$ can occur as a composition factor of an $\ell$-Brauer $m$-rational group for only finitely many pairs $(n, q)$.

To prove Theorem 4.1, we must consider the parity of $n$.

Proposition 5.3.2. Suppose $n$ is even, and assume $(n, q) \neq(4,2),(6,2)$. Define $N=\frac{q^{n-1}+1}{(n, q+1)}$ and $N^{\prime}=\frac{q^{n}-1}{(q+1)(n, q+1)}$. Then, there exists a liftable irreducible $\ell-$ Brauer character $\alpha \in \operatorname{IBr}_{\ell}(S)$ with either $[\mathbb{Q}(\alpha): \mathbb{Q}]=\frac{\phi(N)}{n-1}$ or $[\mathbb{Q}(\alpha): \mathbb{Q}]=\frac{\phi\left(N^{\prime}\right)}{n}$. Further, for a fixed $m \in \mathbb{N}$, only finitely many pairs $(n, q)$ satisfy $\frac{\phi(N)}{n-1}|m| \operatorname{Out}(S) \mid$ or $\frac{\phi\left(N^{\prime}\right)}{n}|m| \operatorname{Out}(S) \mid$. Hence, $\operatorname{PSU}_{n}(q)$ with $n$ even can occur as a composition factor of an $\ell$-Brauer m-rational group for only finitely many pairs $(n, q)$.

Proof. The proof is nearly identical to that of the linear groups. Define $s$ (resp. $\left.s^{\prime}\right)$ to be a generator of the unique subgroup of index $(n, q+1)$ of a cyclic maximal torus $T^{*}$ (resp. $T^{* \prime}$ ) of order $q^{n-1}+1\left(\operatorname{resp} . \frac{q^{n}-1}{q+1}\right)$ in $L^{*}=\operatorname{PGU}_{n}(q)$, the dual of $L=\mathrm{SU}_{n}(q)$, [5, Theorem 2.2]. Let $r$ be a primitive prime divisor of $q^{2(n-1)}-1$ (resp. 
$r^{\prime}$, a primitive prime divisor of $q^{n}-1$ ). Following the proof of Lemma 5.2.3, we see that generators of $\langle s\rangle$ lie in $\frac{\phi(N)}{n-1}$ distinct $L^{*}$-conjugacy classes, as $W\left(T^{*}\right) \cong C_{n-1}$ is cyclic of order $n-1$, and generators of $\left\langle s^{\prime}\right\rangle$ lie in $\frac{\phi\left(N^{\prime}\right)}{n}$ distinct $L^{*}$-conjugacy classes, as $W\left(T^{* \prime}\right) \cong C_{n}$ is cyclic of order $n$ [5, Theorem 2.2]. Note that $\left(N, N^{\prime}\right)=1$ by Lemma 5.1.8, and in particular, $\chi_{s}^{\circ}$ or $\chi_{s^{\prime}}^{\circ}$ is an irreducible $\ell$-Brauer character of $S$. Therefore, $[\mathbb{Q}(\alpha): \mathbb{Q}]=\frac{\phi(N)}{n-1}$ or $[\mathbb{Q}(\alpha): \mathbb{Q}]=\frac{\phi\left(N^{\prime}\right)}{n}$, where $\alpha:=\chi_{s}^{\circ}$ if $\ell \nmid|s|$ or $\alpha:=\chi_{s^{\prime}}^{\circ}$ if $\ell \nmid\left|s^{\prime}\right|$.

As in the linear group case, comparing $\frac{\phi(N)}{n-1}$ and $\frac{\phi\left(N^{\prime}\right)}{n}$ to square root functions, we have

$$
\frac{\phi(N)}{n-1} \geq \sqrt{\frac{q^{n-1}}{2 n^{3}}} \geq \sqrt{\frac{2^{n-2}}{n^{3}}} \quad \text { and } \quad \frac{\phi\left(N^{\prime}\right)}{n} \geq \sqrt{\frac{q^{n-2}}{2 n^{3}}} \geq \sqrt{\frac{2^{n-3}}{n^{3}}}
$$

Further, we see that $m|\operatorname{Out}(S)|=m(n, q+1) f \leq 2 m n q\left(\right.$ recall that $\left.q^{2}=p^{f}\right)$ [29, Table 5.1.A]. Clearly, there exists $n_{0}$ large enough so that $\sqrt{\frac{2^{n-7}}{n^{5}}}>m$ for all $n \geq n_{0}$, and in particular, $\operatorname{PSU}_{n}(q)$ cannot occur as a composition factor of any $\ell$-Brauer $m$-rational group for $n \geq n_{0}$ ( $n$ even) and $q \geq 2$.

Now, for a fixed $4 \leq n \leq n_{0}$, we see that

$$
\sqrt{\frac{q^{n-1}}{8 n^{5}\left(\log _{2}(q)\right)^{2}}} \quad \text { and } \quad \sqrt{\frac{q^{n-2}}{8 n^{5}\left(\log _{2}(q)\right)^{2}}}
$$

are positive and increasing unboundedly for all $q \geq 2$. Hence, for each $4 \leq n \leq n_{0}$, there exists $q_{0}$ such that

$$
\sqrt{\frac{q^{n-1}}{8 n^{5}\left(\log _{2}(q)\right)^{2}}}>m \quad \text { and } \quad \sqrt{\frac{q^{n-2}}{8 n^{5}\left(\log _{2}(q)\right)^{2}}}>m
$$

for all $q \geq q_{0}$, and $\operatorname{PSU}_{n}(q)$ cannot occur as a composition factor of any $\ell$-Brauer $m$-rational group for $4 \leq n \leq n_{0}$ even and $q \geq q_{0}$. Therefore, $\operatorname{PSU}_{n}(q)$ with $n$ even can occur as a composition factor of an $\ell$-Brauer $m$-rational group for only finitely many pairs $(n, q)$. 
Proposition 5.3.3. Suppose $n$ is odd, $(n, q) \neq(7,2)$. Define $N=\frac{q^{n}+1}{(n, q+1)(q+1)}$ and $N^{\prime}=\frac{q^{n-1}-1}{(n, q+1)}$. Then, there exists an irreducible character $\alpha \in \operatorname{IBr}_{\ell}(S)$ with $[\mathbb{Q}(\alpha): \mathbb{Q}]=\frac{\phi(N)}{n}$ or $[\mathbb{Q}(\alpha): \mathbb{Q}]=\frac{\phi\left(N^{\prime}\right)}{n-1}$. Further, for a fixed $m \in \mathbb{N}$, only finitely many pairs $(n, q)$ satisfy $\frac{\phi(N)}{n}|m| \operatorname{Out}(S) \mid$ or $\frac{\phi\left(N^{\prime}\right)}{n-1}|m| \operatorname{Out}(S) \mid$. Hence, $\operatorname{PSU}_{n}(q)$ with $n$ odd can occur as a composition factor of an $\ell$-Brauer m-rational group for only finitely many pairs $(n, q)$.

A Zsigmondy prime for $q^{n}-1$ does not exist when $n=3$ and $q$ is a Mersenne prime, and thus we first prove this proposition for $n=3$.

Lemma 5.3.4. Proposition 5.3.3 holds when $n=3$.

Proof. Using CHEVIE, [19], we see there exist irreducible ordinary characters $\alpha_{1}$ and $\alpha_{2}$ of degrees $(q-1)(q+1)^{2}$ and $(q+1)\left(q^{2}-q+1\right)$, respectively. Note that these are both semisimple characters corresponding to semisimple elements of coprime order (recall that $\operatorname{PSU}_{3}(2)$ is not simple, and thus we assume $q>2$ ), and hence, at least one is an irreducible $\ell$-Brauer character when restricted to the $\ell$-regular classes. Further, they take on the values $\pm\left(\phi_{6}+\phi_{6}^{-q}+\phi_{6}^{q^{2}}\right)$, where $\phi_{6}$ is a primitive $\frac{q^{3}+1}{(3, q+1)(q+1)}$-th root of unity and $\pm\left(\xi_{2}+\xi_{2}^{-q}\right)$, where $\xi_{2}$ is a primitive $\frac{q^{3-1}-1}{(3, q+1)}$-th root of unity. Thus,

$$
\frac{\phi\left(\frac{q^{3}+1}{(q+1)(3, q+1)}\right)}{3} \mid\left[\mathbb{Q}\left(\alpha_{1}\right): \mathbb{Q}\right], \quad \text { or } \quad \frac{\phi\left(\frac{q^{3-1}-1}{(3, q+1)}\right)}{2} \mid\left[\mathbb{Q}\left(\alpha_{2}\right): \mathbb{Q}\right]
$$

Now, we may prove Proposition 5.3.3 for $n \geq 5,(n, q) \neq(7,2)$ :

Proof. Assume $n \geq 5$ and $n \geq 5,(n, q) \neq(7,2)$. Define $s$ (resp. $\left.s^{\prime}\right)$ to be a generator of the unique subgroup of index $(n, q+1)$ of a cyclic maximal torus $T^{*}$ (resp. $\left.T^{* \prime}\right)$ of order $\frac{q^{n}+1}{q+1}\left(\operatorname{resp} . q^{n-1}-1\right)$ in $L^{*}=\operatorname{PGU}_{n}(q)$, the dual of $L=\operatorname{SU}_{n}(q)$, [5, Theorem 2.2 ]. Let $r$ be a primitive prime divisor of $q^{2 n}-1$ (resp. $r^{\prime}$ a primitive prime divisor 
of $q^{n-1}-1$ ). Following the proof of Lemma 5.2.3, we see that generators of $\langle s\rangle$ (resp. $\left.\left\langle s^{\prime}\right\rangle\right)$ lie in $\frac{\phi(N)}{n}$ (resp. $\left.\frac{\phi\left(N^{\prime}\right)}{n-1}\right)$ distinct $L^{*}$-conjugacy classes, as $W\left(T^{*}\right) \cong C_{n}$ is cyclic of order $n$ (resp. $\left.W\left(T^{* \prime}\right) \cong C_{n-1}\right)$, [5, Theorem 2.2]. Note that, as in the linear group case, we must consider the case $r^{\prime}=n$ separately, as (in the notation of Lemma 5.2.3) the element $t^{\prime}$ may not have a connected centralizer. However, if $r^{\prime}=n$ is prime, then $Z(L)=1$ as $n=r^{\prime} \nmid q+1$, and $L \cong L^{*} \cong \operatorname{PSU}_{n}(q)$. Thus, we may use $\mathcal{G}=\operatorname{PGU}_{n}\left(\overline{\mathbb{F}}_{p}\right)$ and $\mathcal{G}^{*}=\mathrm{SU}_{n}\left(\overline{\mathbb{F}}_{p}\right)$. Then, choosing our element $t^{\prime}$ as before, $C_{\mathcal{G}^{*}}\left(t^{\prime}\right)$ is connected as $\mathcal{G}$ has connected center ([6, Theorem 4.5.9]), and the proof proceeds as in the case $r^{\prime} \geq n+1$.

Now, we have that $\left(N, N^{\prime}\right)=1$ by Lemma 5.1.8, and hence, either $\chi_{s}^{\circ}$ or $\chi_{s^{\prime}}^{\circ}$ is an irreducible $\ell$-Brauer character of $S$. Therefore, $[\mathbb{Q}(\alpha): \mathbb{Q}]=\frac{\phi(N)}{n}$ or $[\mathbb{Q}(\alpha): \mathbb{Q}]=$ $\frac{\phi\left(N^{\prime}\right)}{n-1}$, where $\alpha:=\chi_{s}^{\circ}$ if $\ell \nmid|s|$ or $\alpha:=\chi_{s^{\prime}}^{\circ}$ if $\ell \nmid\left|s^{\prime}\right|$.

As in the linear group case, comparing $\frac{\phi(N)}{n}$ and $\frac{\phi\left(N^{\prime}\right)}{n-1}$ to square root functions, we have

$$
\frac{\phi(N)}{n} \geq \sqrt{\frac{q^{n-2}}{2 n^{3}}} \geq \sqrt{\frac{2^{n-3}}{n^{3}}} \quad \text { and } \quad \frac{\phi\left(N^{\prime}\right)}{n-1} \geq \sqrt{\frac{q^{n-2}}{2 n^{3}}} \geq \sqrt{\frac{2^{n-3}}{n^{3}}} .
$$

Further, we see that $m|\operatorname{Out}(S)|=m(n, q+1) f \leq 2 m n q\left(\right.$ recall that $\left.q^{2}=p^{f}\right)$ [29, Table 5.1.A]. Clearly, there exists $n_{0}$ large enough so that $\sqrt{\frac{2^{n-7}}{n^{5}}}>m$ for all $n \geq n_{0}$, and in particular, $\operatorname{PSU}_{n}(q)$ cannot occur as a composition factor of any $\ell$-Brauer $m$-rational group for $n \geq n_{0}(n$ odd) and $q \geq 2$.

Now, for a fixed $3 \leq n \leq n_{0}$, we see that $\sqrt{\frac{q^{n-2}}{8 n^{5}\left(\log _{2}(q)\right)^{2}}}$ is positive and increasing unboundedly for all $q \geq 8$. Hence, for each $3 \leq n \leq n_{0}$, there exists $q_{0}$ such that $\sqrt{\frac{q^{n-2}}{8 n^{5}\left(\log _{2}(q)\right)^{2}}}>m$ for all $q \geq q_{0}$, and $\operatorname{PSU}_{n}(q)$ cannot occur as a composition factor of any $\ell$-Brauer $m$-rational group for $3 \leq n \leq n_{0}$ odd and $q \geq q_{0}$. Therefore, $\operatorname{PSU}_{n}(q)$ with $n$ odd can occur as a composition factor of an $\ell$-Brauer $m$-rational group for only finitely many pairs $(n, q)$. 
Corollary 5.3.5. $S=\operatorname{PSU}_{n}(q)$ with $\ell \nmid q$ can occur as a composition factor of an $\ell$-Brauer rational group if and only if

$$
(n, q) \in\{(3,3),(3,5),(4,2),(4,3)\}
$$

Proof. Fix $m=1$. First, we observe that a primitive prime divisor of $2^{6}-1$ does not exist, and thus, we handle the cases $S=\mathrm{U}_{4}(2), \mathrm{U}_{6}(2)$, and $\mathrm{U}_{7}(2)$ separately. Using GAP, we see that $\mathrm{U}_{4}(2) .2$ is rational, and thus $\mathrm{U}_{4}(2)$ occurs as a composition factor of an $\ell$-Brauer rational group for any $\ell$.

We have that the (non-defining characteristic) primes dividing $\left|\mathrm{U}_{6}(2)\right|$ are 3,5 , 7, and 11. Note that all such $\ell$-modular characters are available in GAP. When $\ell \neq 3$ (and, of course, $\ell \neq 2$ ), we apply the same argument as $\mathrm{L}_{2}(7)$. We have that $N_{G}\left(S_{1}\right) / C_{G}\left(S_{1}\right) \cong S, S .2, S .3$, or $S . S_{3}$. In the case that $N_{G}\left(S_{1}\right) / C_{G}\left(S_{1}\right) \cong S$, use an irrational character of degree 770. In any of the latter cases, we see that a rational character of degree 560 extends to a liftable irrational character of $S .2, S .3$, and $S . S_{3}$. Therefore, $\mathrm{U}_{6}(2)$ cannot occur as a composition factor of any $\ell$-Brauer rational group for $\ell \neq 2,3$. When $\ell=3$, we apply the same argument as $\mathrm{L}_{2}(7)$. When $N_{G}\left(S_{1}\right) / C_{G}\left(S_{1}\right) \cong S$ or $S .3$, choose an irrational liftable character of degree 25515 . When $N_{G}\left(S_{1}\right) / C_{G}\left(S_{1}\right) \cong S .2$ or $S . S_{3}$, choose a rational character of degree 560 that extends to a liftable irrational character of $S .2$ and $S . S_{3}$. Thus, $\mathrm{U}_{6}(2)$ cannot occur as a composition factor of a 3-Brauer rational group.

Using GAP, we see that $S=\mathrm{U}_{7}(2)$ does, in fact, have the semisimple characters $\alpha_{1}$ and $\alpha_{2}$ corresponding to elements of orders $\frac{2^{7}+1}{2+1}=43$ and $2^{7-1}-1=63$, respectively, as described in Propositions 5.3.3 and 5.3.2. Notice that $(43,63)=1$ and therefore, either $\alpha_{1}$ or $\alpha_{2}$ restricts irreducibly to the $\ell$-regular classes of $S$ for any $\ell>2$. However, neither satisfy the conclusion of Theorem 5.1 .1 , as $6=\frac{\phi(43)}{7}=$ $\left[\mathbb{Q}\left(\alpha_{1}\right): \mathbb{Q}\right] \nmid 2=|\operatorname{Out}(S)|$ and $6=\frac{\phi(63)}{6}=\left[\mathbb{Q}\left(\alpha_{2}\right): \mathbb{Q}\right] \nmid 2=|\operatorname{Out}(S)|$. Therefore, $\mathrm{U}_{7}(2)$ cannot occur as a composition factor of any $\ell$-Brauer rational group for $\ell>2$. Now, from Propositions 5.3 .2 and 5.3.3. we must solve the relations $\frac{\phi\left(\frac{q^{n}+1}{(n, q+1)(q+1)}\right)}{n}$ । 
$|\operatorname{Out}(S)|$ or $\frac{\phi\left(\frac{q^{n-1}-1}{(n, q+1)}\right)}{n-1}|| \operatorname{Out}(S) \mid$ for $n$ odd and $\frac{\phi\left(\frac{q^{n-1}+1}{(n, q+1)}\right)}{n-1}|| \operatorname{Out}(S)\left|\operatorname{or} \frac{\phi\left(\frac{q^{n}-1}{(n, q+1)(q+1)}\right)}{n}\right|$ $|\operatorname{Out}(S)|$ for $n$ even, and $(n, q) \neq(4,2),(6,2),(7,2)$. In the notation of the proofs of Propositions 5.3 .2 and 5.3 .3 , we find that $n_{0}=34$. For each $n \leq n_{0}$, this yields the following values $q_{0}$ :

\begin{tabular}{|c|c|c|c|c|c|c|c|c|c|c|c|c|c|c|c|c|}
\hline$n$ & 3 & 4 & 5 & 6 & 7 & 8 & 9 & 10 & 11 & 12 & 13 & 14 & 15 & 16 & 17 & 18 \\
\hline$q_{0}$ & 738816 & 887 & 36 & 22 & 13 & 11 & 7 & 7 & 6 & 5 & 5 & 5 & 4 & 4 & 4 & 3 \\
\hline
\end{tabular}

\begin{tabular}{|c|c|c|c|c|c|c|c|c|c|c|c|c|c|c|c|}
\hline$n$ & 19 & 20 & 21 & 22 & 23 & 24 & 25 & 26 & 27 & 28 & 29 & 30 & 31 & 32 & 33 \\
\hline$q_{0}$ & 3 & 3 & 3 & 3 & 3 & 3 & 3 & 3 & 3 & 3 & 3 & 3 & 3 & 3 & 3 \\
\hline
\end{tabular}

Verifying which of these (finitely many) pairs $(n, q)$ satisfy $\frac{\phi\left(\frac{q^{n}+1}{(n, q+1)(q+1)}\right)}{n}|| \operatorname{Out}(S) \mid$ or $\frac{\phi\left(\frac{q^{n-1}-1}{(n, q+1)}\right)}{n-1}|| \operatorname{Out}(S) \mid$ for $n$ odd and $\frac{\phi\left(\frac{q^{n-1}+1}{(n, q+1)}\right)}{n-1}|| \operatorname{Out}(S) \mid$ or $\frac{\phi\left(\frac{q^{n}-1}{(n, q+1)(q+1)}\right)}{n} \mid$ $|\operatorname{Out}(S)|$ for $n$ even, we determine that $S=\operatorname{PSU}_{n}(q)$ can occur as a composition factor of an $\ell$-Brauer rational group only if

$$
(n, q) \in\{(3,3),(3,4),(3,5),(3,8),(4,3),(5,2)\}
$$

We have that the (non-defining characteristic) primes dividing $\left|\mathrm{U}_{3}(3)\right|$ are 2 and 7. Using GAP, we see that $\mathrm{U}_{3}(3) .2$ is 2 -Brauer rational. To show that $\mathrm{U}_{3}(3)$ cannot occur as a composition factor of any 7-Brauer or $\ell$-Brauer rational group where $\ell \nmid \mid \mathrm{U}_{3}(3)$, apply the same argument as in $\mathrm{L}_{2}(7)$. In the case that $N_{G}\left(S_{1}\right) / C_{G}\left(S_{1}\right) \cong S$, use an irrational irreducible character of degree 7 , and in the case that $N_{G}\left(S_{1}\right) / C_{G}\left(S_{1}\right) \cong S .2$, use a rational character of $S$ of degree 6 that extends to an irrational liftable character of $S .2$.

We have that the (non-defining characteristic) primes dividing $\left|\mathrm{U}_{3}(4)\right|$ are 3 and 5 and 13. To show that $\mathrm{U}_{3}(4)$ cannot occur as a composition factor of any 13-Brauer or $\ell$-Brauer rational group where $\ell \nmid\left|\mathrm{U}_{3}(4)\right|$, apply the same $\operatorname{argument}$ as in $\mathrm{L}_{2}(7)$. In 
the case that $N_{G}\left(S_{1}\right) / C_{G}\left(S_{1}\right) \cong S$, use an irrational irreducible character of degree 13 , and in the case that $N_{G}\left(S_{1}\right) / C_{G}\left(S_{1}\right) \cong S .2$ or $N_{G}\left(S_{1}\right) / C_{G}\left(S_{1}\right) \cong S .4$, use the rational character of $S$ of degree 12 that extends to an irrational liftable character of $S .2$ and S.4. The same argument, replacing the character of degree 13 with the character of degree 75 in the case that $N_{G}\left(S_{1}\right) / C_{G}\left(S_{1}\right) \cong S$ shows that $\mathrm{U}_{3}(4)$ cannot occur as a composition factor of any 3-Brauer or 5-Brauer rational group.

We have that the (non-defining characteristic) primes dividing $\left|\mathrm{U}_{3}(5)\right|$ are 2,3 , and 7. Using GAP, we see that $\mathrm{U}_{3}(5) .2$ is 2-Brauer rational. To show that $\mathrm{U}_{3}(5)$ cannot occur as a composition factor of any 3-Brauer, 7-Brauer, or $\ell$-Brauer rational group where $\ell \nmid\left|\mathrm{U}_{3}(5)\right|$, apply the same argument as in $\mathrm{L}_{2}(7)$. In the case that $N_{G}\left(S_{1}\right) / C_{G}\left(S_{1}\right) \cong S$ or $N_{G}\left(S_{1}\right) / C_{G}\left(S_{1}\right) \cong S .3$, use an irrational irreducible character of degree 126, and in the case that $N_{G}\left(S_{1}\right) / C_{G}\left(S_{1}\right) \cong S .2$ or $N_{G}\left(S_{1}\right) / C_{G}\left(S_{1}\right) \cong$ $S . S_{3}$, use a rational character of $S$ of degree 20 that extends to an irrational liftable character of $S .2$ and S.S $S_{3}$.

We have that the (non-defining characteristic) primes dividing $\left|\mathrm{U}_{3}(8)\right|$ are 3,7 , and 19. To show that $\mathrm{U}_{3}(8)$ cannot occur as a composition factor of any 3-Brauer, 7-Brauer, 19-Brauer, or $\ell$-Brauer rational group where $\ell \nmid\left|\mathrm{U}_{3}(8)\right|$, apply the same argument as in $\mathrm{L}_{2}(7)$. In the case that $N_{G}\left(S_{1}\right) / C_{G}\left(S_{1}\right) \cong S$, use an irrational character of degree 57 (if the prime is not 3) or 513 (if the prime is 3). In the case that $N_{G}\left(S_{1}\right) / C_{G}\left(S_{1}\right) \cong S .2, N_{G}\left(S_{1}\right) / C_{G}\left(S_{1}\right) \cong S . S_{3}$, or $N_{G}\left(S_{1}\right) / C_{G}\left(S_{1}\right) \cong \operatorname{Aut}(S)$, use a rational character of $S$ of degree 56 that extends to an irrational liftable character of $S .2, S . S_{3}$, and $\operatorname{Aut}(S)$. In the case that $N_{G}\left(S_{1}\right) / C_{G}\left(S_{1}\right) \cong S .3_{1}, N_{G}\left(S_{1}\right) / C_{G}\left(S_{1}\right) \cong$ $S .3_{2}$, or $N_{G}\left(S_{1}\right) / C_{G}\left(S_{1}\right) \cong S .3_{3}$, use a rational character of $S$ of degree 56 (if the prime is not 3), or an irrational character of degree 1701 (if the prime is 3 and $\left.N_{G}\left(S_{1}\right) / C_{G}\left(S_{1}\right) \neq S .3_{2}\right)$ or 513 (if the prime is 3 and $N_{G}\left(S_{1}\right) / C_{G}\left(S_{1}\right) \cong S .3_{2}$ ) that extends to an irrational liftable character of $S .3_{1}, S .3_{2}$, and $S .3_{3}$.

We have that the (non-defining characteristic) primes dividing $\left|\mathrm{U}_{4}(3)\right|$ are 2,5 , and 7. Using GAP, we see that $\mathrm{U}_{4}(3) . D_{8}$ is 2 -Brauer rational and 7-Brauer rational. 
To show that $\mathrm{U}_{4}(3)$ cannot occur as a composition factor of any 5 -Brauer or $\ell$-Brauer rational group where $\ell \nmid\left|\mathrm{U}_{4}(3)\right|$, apply the same argument as in $\mathrm{L}_{2}(7)$. In the case that $N_{G}\left(S_{1}\right) / C_{G}\left(S_{1}\right) \cong S .2_{1}, N_{G}\left(S_{1}\right) / C_{G}\left(S_{1}\right) \cong S .2_{2}, N_{G}\left(S_{1}\right) / C_{G}\left(S_{1}\right) \cong S .2_{3}$, or $N_{G}\left(S_{1}\right) / C_{G}\left(S_{1}\right) \cong S .4$, use a rational character of $S$ of degree 560 that extends to an irrational liftable character of $S .2_{1}, S .2_{2}, S .2_{3}$, and $S .4$. In the case that $N_{G}\left(S_{1}\right) / C_{G}\left(S_{1}\right) \cong S$ or $N_{G}\left(S_{1}\right) / C_{G}\left(S_{1}\right) \cong S . D_{8}$, use an irrational character of $S$ of degree 640 that lies below an irrational liftable character of $S . D_{8}$.

We have that the (non-defining characteristic) primes dividing $\left|\mathrm{U}_{5}(2)\right|$ are 3,5 , and 11 . To show that $\mathrm{U}_{5}(2)$ cannot occur as a composition factor of any 3-Brauer, 5-Brauer, or $\ell$-Brauer rational group where $\ell \nmid\left|\mathrm{U}_{5}(2)\right|$, apply the same argument as in $\mathrm{L}_{2}(7)$. In the case that $N_{G}\left(S_{1}\right) / C_{G}\left(S_{1}\right) \cong S$, use an irreducible character of degree 1215 , and in the case that $N_{G}\left(S_{1}\right) / C_{G}\left(S_{1}\right) \cong S .2$, use a rational character of $S$ of degree 10 that extends to an irrational liftable character of S.2. To show that $\mathrm{U}_{5}(2)$ cannot occur as a composition factor of any 11-Brauer, apply the same argument as in $\mathrm{L}_{2}(7)$. In the case that $N_{G}\left(S_{1}\right) / C_{G}\left(S_{1}\right) \cong S$, use an irrational irreducible character of degree 11 , and in the case that $N_{G}\left(S_{1}\right) / C_{G}\left(S_{1}\right) \cong S .2$, use a rational character of $S$ of degree 10 that extends to an irrational liftable character of S.2.

\begin{tabular}{|c|c|c|c|c|}
\hline$(n, q)$ & $(3,3)$ & $(3,5)$ & $(4,2)$ & $(4,3)$ \\
\hline Prime(s) $\ell$ & 2 & 2 & Any prime $\ell$ & 2,7 \\
\hline$\ell$-Brauer Rational Group & $\mathrm{U}_{3}(3) .2$ & $\mathrm{U}_{3}(5) .2$ & $\mathrm{U}_{4}(2) .2$ & $\mathrm{U}_{4}(3) . D_{8}$ \\
\hline
\end{tabular}

\subsection{The Symplectic and Odd-Dimensional Orthogonal Groups}

Theorem 5.4.1. Let $S=\operatorname{PSp}_{2 n}(q)$ with $n \geq 2$ and $(n, q) \neq(3,2),(6,2)$, or $S=$ $\Omega_{2 n+1}(q)$ with $n>2, q=p^{f}, p \neq \ell$. Then $S$ can occur as a composition factor of an $\ell$-Brauer m-rational group for only finitely many pairs $(n, q)$. 
Before we prove Theorem 5.4.1, we recall that no Zsigmondy prime exists for $q^{2}-1$ when $q$ is a Mersenne prime, and thus, we must prove the following lemma.

Lemma 5.4.2. Let $S=S_{4}(q)$. Then there exist characters $\alpha_{1}, \alpha_{2} \in \operatorname{IBr}_{\ell}(S)$ satisfying

$$
\frac{\phi\left(\frac{q^{2}+1}{(2, q-1)}\right)}{4} \mid\left[\mathbb{Q}\left(\alpha_{1}\right): \mathbb{Q}\right] \quad \text { or } \quad \frac{\phi\left(\frac{q^{2}-1}{(2, q-1)}\right)}{4} \mid\left[\mathbb{Q}\left(\alpha_{2}\right): \mathbb{Q}\right] \text {. }
$$

Furthermore, Theorem 5.4.1 holds for $n=2$.

Proof. The ordinary character tables of $\operatorname{Sp}_{4}(q)$ are known (see [43] for $q$ odd and [11] for $q$ even). We see that there are irreducible semisimple characters $\chi_{1}$ and $\chi_{2}$ (with $\operatorname{ker}\left(\chi_{i}\right) \geq Z\left(\operatorname{Sp}_{4}(q)\right)$ for $\left.i=1,2\right)$ of degree $\left(q^{2}-1\right)^{2}$ and $q^{4}-1$, respectively, that correspond to semisimple elements of order $\frac{q^{2}-1}{(2, q-1)}$ and $\frac{q^{2}+1}{(2, q-1)}$, respectively. Thus, we may view these as ordinary irreducible characters of $\mathrm{S}_{4}(q)$, and as these orders are coprime, the restriction of $\chi_{1}$ or $\chi_{2}$ to the $\ell$-regular classes of $\mathrm{S}_{4}(q)$ is an irreducible $\ell$-Brauer character of $\mathrm{S}_{4}(q)$. Further, these take on the values $\pm\left(\xi+\xi^{-1}+\xi^{q}+\xi^{-q}\right)$, where $\xi$ is a primitive $\frac{q^{2}-1}{(2, q-1)}$-th root of unity, and $\pm\left(\zeta+\zeta^{-1}+\zeta^{q}+\zeta^{-q}\right)$, where $\zeta$ is a primitive $\frac{q^{2}+1}{(2, q-1)}$-th root of unity, respectively. Therefore, $\alpha_{1}:=\chi_{1}^{\circ}$ and $\alpha_{2}:=\chi_{2}^{\circ}$ satisfy either

$$
\frac{\phi\left(\frac{q^{2}+1}{(2, q-1)}\right)}{4} \mid\left[\mathbb{Q}\left(\alpha_{1}\right): \mathbb{Q}\right] \quad \text { or } \quad \frac{\phi\left(\frac{q^{2}-1}{(2, q-1)}\right)}{4} \mid\left[\mathbb{Q}\left(\alpha_{2}\right): \mathbb{Q}\right] .
$$

Now, for a fixed $m$, we see that

$$
\begin{aligned}
& \frac{\phi\left(\frac{q^{2}+1}{(2, q-1)}\right)}{4} \geq \sqrt{\frac{q^{2}+1}{64}>4 m \log _{2}(q) \geq m\left|\operatorname{Out}\left(\mathrm{S}_{4}(q)\right)\right|} \\
& \quad \text { and } \\
& \frac{\phi\left(\frac{q^{2}-1}{(2, q-1)}\right)}{4} \geq \sqrt{\frac{q^{2}-1}{64}}>4 m \log _{2}(q) \geq m\left|\operatorname{Out}\left(\mathrm{S}_{4}(q)\right)\right|
\end{aligned}
$$

for all but finitely many $q$, and therefore, by Theorem 5.1.1, $\mathrm{S}_{4}(q)$ can occur as a composition factor of an $\ell$-Brauer $m$-rational group for only finitely many $q$. 
Now, we may assume $n>2$ and proceed to prove Theorem 5.4.1.

Proof. Let $L=\operatorname{Sp}_{2 n}(q)$ (resp., $\left.L=\operatorname{Spin}_{2 n+1}(q)\right), n>2$, so that $L^{*}=\mathrm{SO}_{2 n+1}$ (resp., $\left.L^{*}=\operatorname{PCSp}_{2 n}(q)\right)$. Define $s$ to be a generator of the unique subgroup of index $(2, q-1)$ (resp., $\left.\left(q^{n}+1\right)_{2}\right)$ in a cyclic maximal torus $T^{*}$ of order $q^{n}+1$ in $L^{*}$, and $s^{\prime}$ to be a generator of the unique subgroup of index $(2, q-1)$ (resp., $\left.(q-1)_{2}\right)$ in a cyclic maximal torus $T^{* \prime}$ of order $q^{n}-1$ in $L^{*}$ [5, Theorem 3], [50, Theorem 1]. Let $r$ be a primitive prime divisor of $q^{2 n}-1$. As in the proof of Lemma 5.2.3, it follows that generators of $\langle s\rangle$ lie in $\frac{\phi(N)}{2 n}$ distinct $L^{*}$-conjugacy classes, where $N=\frac{q^{n}+1}{(2, q-1)}$ (resp., $\left.N=\frac{q^{n}+1}{\left(q^{n}+1\right)_{2}}\right)$, as $W\left(T^{*}\right) \cong C_{2 n}$, [5, Theorem 3], [50, Theorem 1].

Now, we see that $s^{\prime}$ is semisimple as $p \nmid\left|s^{\prime}\right|$. Let $s^{\prime}$ act on the natural module $V=$ $\mathbb{F}_{q}^{2 n}$ (resp. $\mathbb{F}_{q}^{2 n+1}$ ). Then, the eigenvalues are $\lambda, \lambda^{q}, \ldots, \lambda^{q^{n-1}}, \lambda^{-1}, \ldots, \lambda^{-q^{n-1}}$ (resp. $\left.\lambda, \lambda^{q}, \ldots, \lambda^{q^{n-1}}, \lambda^{-1}, \ldots, \lambda^{-q^{n-1}}, 1\right)$, where $\lambda$ is a primitive $\frac{q^{n}-1}{(2, q-1)}$-th (resp. $\frac{q^{n}-1}{(q-1)_{2}}$-th) root of unity. We claim that, as all eigenvalues are necessarily distinct, $s^{\prime}$ is a regular element:

Lemma 5.4.3. Let $s^{\prime} \in L^{*}$ be semisimple of odd order with no multiple eigenvalues. Then $s^{\prime}$ is regular.

Proof. Let $\mathcal{H}=\mathrm{Sp}_{2 n}\left(\overline{\mathbb{F}}_{p}\right)$ (resp. $\mathrm{SO}_{2 n+1}\left(\overline{\mathbb{F}}_{p}\right)$ ), $F$ be a Frobenius morphism, and $\mathcal{G}^{*}$ be such that $\mathcal{G}^{* F}=L^{*}$ and $\mathcal{H} / Z=\mathcal{G}^{*}$, where $Z \leq C_{2}$. From Theorem 3.5.3 of [6], we see that $C_{\mathcal{H}}\left(s^{\prime}\right)^{\circ}=\left\langle\mathcal{T}, X_{\alpha}, \alpha\left(s^{\prime}\right)=1\right\rangle$, where $X_{\alpha}$ are the root subgroups. Suppose that there exists some root $\alpha$ such that $\alpha\left(s^{\prime}\right)=1$. Then $-\alpha\left(s^{\prime}\right)=1$ as well, and $\mathrm{SL}_{2}\left(\overline{\mathbb{F}}_{p}\right) \cong\left\langle X_{\alpha}, X_{-\alpha}\right\rangle \leq C_{\mathcal{H}}\left(s^{\prime}\right)^{\circ}$. However, $C_{\mathcal{H}}\left(s^{\prime}\right)^{\circ}$ is abelian as there are no multiple eigenvalues: the natural module decomposes as $V=V_{1} \oplus \cdots \oplus V_{n} \oplus V_{1}^{\prime} \oplus \cdots \oplus V_{n}^{\prime}$ (resp. $V=V_{1} \oplus \cdots \oplus V_{n} \oplus V_{1}^{\prime} \oplus \cdots \oplus V_{n}^{\prime} \oplus V^{\prime}$ ) where $\left.s^{\prime}\right|_{V_{i}}=\lambda^{q^{i-1}}$ and $\left.s^{\prime}\right|_{V_{i}^{\prime}}=\lambda^{-q^{i-1}}$ (and $s^{\prime}$ acts trivially on $V^{\prime}$ ) in the notation above. Hence, $C_{\mathcal{H}}\left(s^{\prime}\right)^{\circ}$ cannot contain a non-abelian subgroup. Thus, no such $\alpha$ can exist, and $C_{\mathcal{H}}\left(s^{\prime}\right)^{\circ}=\mathcal{T}$. Now, let $\mathcal{D}$ be the full preimage of $C_{\mathcal{G}^{*}}\left(s^{\prime}\right)$ in $\mathcal{H}$. Then, as $\left|s^{\prime}\right|$ is odd (in particular, coprime to 
$|Z|)$, we have that $\mathcal{D}=C_{\mathcal{H}}\left(s^{\prime}\right)$ : there is a unique element of $\mathcal{D}$ of order $\left|s^{\prime}\right|$ in the preimage of $s^{\prime}$, which we also denote $s^{\prime}$. Further, if $s^{\prime \prime}$ is an element of order $\left|s^{\prime}\right|, s^{\prime \prime}$ is conjugate to $s^{\prime}$ in $\mathcal{G}^{*}$ if and only if the preimages $s^{\prime \prime}$ and $s^{\prime}$ are conjugate in $\mathcal{H}$, and thus, $\mathcal{D}=C_{\mathcal{H}}\left(s^{\prime}\right)$. Hence, as $\mathcal{D}^{\circ}$ is a maximal torus in $\mathcal{H}, C_{\mathcal{G}^{*}}\left(s^{\prime}\right)^{\circ}$ is a maximal torus of $\mathcal{G}^{*}$, and $s^{\prime}$ is a regular element.

Finally, to show that generators of $\left\langle s^{\prime}\right\rangle$ lie in $\frac{\phi\left(N^{\prime}\right)}{2 n}$ distinct $L^{*}$-conjugacy classes, where $N^{\prime}=\frac{q^{n}-1}{(2, q-1)}$ (resp., $\left.N^{\prime}=\frac{q^{n}-1}{(q-1)_{2}}\right)$, we prove the following lemma:

Lemma 5.4.4. Let $\mathcal{G}$ be connected reductive with Frobenius morphism $F, \mathcal{T}$ a maximal torus. Suppose $x, y \in T:=\mathcal{T}^{F}$ are regular $\left(\right.$ so that $\left.C_{\mathcal{G}}(x)^{\circ}=\mathcal{T}=C_{\mathcal{G}}(y)^{\circ}\right)$. If $x, y$ are conjugate in $G:=\mathcal{G}^{F}$, then $x, y$ are conjugate under $N_{\mathcal{G}}(\mathcal{T})^{F} / \mathcal{T}^{F}$.

Proof. Suppose $x=g y g^{-1}$ for some $g \in G$. Let $t \in \mathcal{T}$. We claim that $g t g^{-1} \in \mathcal{T}$ so that $g \in\left(N_{\mathcal{G}}(\mathcal{T})\right)^{F}$. As $y$ is regular, $\mathcal{T}=C_{\mathcal{G}}(y)^{\circ}$, and thus, $t=y t y^{-1}$. Thus,

$$
g t g^{-1}=g y t y^{-1} g^{-1}=g y g^{-1} g t g^{-1} g y^{-1} g^{-1}=x g t g^{-1} x^{-1}
$$

That is, $g t g^{-1} \in C_{\mathcal{G}}(x)$. Further, $g C_{\mathcal{G}}(y)^{\circ} g^{-1}$ is connected and contains the identity of $\mathcal{G}$. Hence,

$$
g t g^{-1} \in C_{\mathcal{G}}(x)^{\circ}=\mathcal{T}
$$

Therefore, $g \in\left(N_{\mathcal{G}}(\mathcal{T})\right)^{F}$, and quotienting out by $\mathcal{T}^{F}$, we have $x$ and $y$ are conjugate under $N_{\mathcal{G}}(\mathcal{T})^{F} / \mathcal{T}^{F}$.

As $W\left(T^{* \prime}\right) \cong C_{2 n}$, [5, Theorem 3], [50, Theorem 1], generators of $\left\langle s^{\prime}\right\rangle$ lie in $\frac{\phi\left(N^{\prime}\right)}{2 n}$ distinct $L^{*}$-conjugacy classes. Note that $\left(N, N^{\prime}\right)=1$, and in particular, either $s$ or $s^{\prime}$ is an $\ell^{\prime}$-element. Therefore, $\left[\mathbb{Q}\left(\chi_{s}^{\circ}\right): \mathbb{Q}\right]=\frac{\phi(N)}{2 n}$ if $\ell \nmid|s|$ or $\left[\mathbb{Q}\left(\chi_{s^{\prime}}^{\circ}\right): \mathbb{Q}\right]=\frac{\phi\left(N^{\prime}\right)}{2 n}$ if $\ell \nmid\left|s^{\prime}\right|$.

From Theorem 5.1.1, we must determine when

$$
\frac{\phi(N)}{2 n}|m| \operatorname{Out}(S) \mid= \begin{cases}2 m(2, q-1) f & q \text { even and } n=2 \\ m(2, q-1) f & \text { else }\end{cases}
$$


or $\frac{\phi\left(N^{\prime}\right)}{2 n}|m| \operatorname{Out}(S) \mid[29$, Table 5.1.A]. As usual, applying Lemma 3.1.7 and Lemma 5.1.9, we compare $\frac{\phi(N)}{2 n}$ and $\frac{\phi\left(N^{\prime}\right)}{2 n}$ to square root functions, allowing us to find $n_{0} \in \mathbb{N}$ such that $\frac{\phi(N)}{2 n}>m|\operatorname{Out}(S)|$ and $\frac{\phi\left(N^{\prime}\right)}{2 n}>m|\operatorname{Out}(S)|$ for all $n \geq n_{0}$ and $q \geq 2$ :

$$
\begin{aligned}
& \frac{\phi(N)}{2 n \cdot 2(2, q-1) f} \geq \sqrt{\frac{q^{n}+1}{256 q^{2} n^{2}}} \geq \sqrt{\frac{2^{n-10}}{n^{2}}} \\
& \text { and } \\
& \frac{\phi\left(N^{\prime}\right)}{2 n \cdot 2(2, q-1) f} \geq \sqrt{\frac{q^{n}-1}{256 q^{2} n^{2}}} \geq \sqrt{\frac{2^{n-11}}{n^{2}}}
\end{aligned}
$$

when $L=\operatorname{Sp}_{2 n}(q)$, and

$$
\begin{gathered}
\frac{\phi(N)}{2 n \cdot 2(2, q-1) f} \geq \sqrt{\frac{q^{n-4}}{128 n^{2}}} \geq \sqrt{\frac{2^{n-11}}{n^{2}}} \\
\text { and } \\
\frac{\phi\left(N^{\prime}\right)}{2 n \cdot 2(2, q-1) f} \geq \sqrt{\frac{q^{n-5}}{128 n^{3}}} \geq \sqrt{\frac{2^{n-12}}{n^{3}}}
\end{gathered}
$$

when $L=\operatorname{Spin}_{2 n+1}(q)$. As in the linear and unitary cases, for each $n<n_{0}$, we may find $q_{0} \in \mathbb{N}$ so that $\frac{\phi(N)}{2 n}>m|\operatorname{Out}(S)|$ and $\frac{\phi\left(N^{\prime}\right)}{2 n}>m|\operatorname{Out}(S)|$ for all $q>q_{0}$ :

$$
\begin{gathered}
\frac{\phi(N)}{2 n \cdot 2(2, q-1) f} \geq \sqrt{\frac{q^{n}}{256 n^{2}\left(\log _{2}(q)\right)^{2}}} \\
\text { and } \\
\frac{\phi\left(N^{\prime}\right)}{2 n \cdot 2(2, q-1) f} \geq \sqrt{\frac{q^{n}-1}{256 n^{2}\left(\log _{2}(q)\right)^{2}}}
\end{gathered}
$$

when $L=\operatorname{Sp}_{2 n}(q)$, and

$$
\begin{gathered}
\frac{\phi(N)}{2 n \cdot 2(2, q-1) f} \geq \sqrt{\frac{q^{n-2}}{128 n^{2}\left(\log _{2}(q)\right)^{2}}} \\
\frac{\phi\left(N^{\prime}\right)}{2 n \cdot 2(2, q-1) f} \geq \sqrt{\frac{q^{n}-1}{128(q+1) n^{3}\left(\log _{2}(q)\right)^{2}}}
\end{gathered}
$$


when $L=\operatorname{Spin}_{2 n+1}(q)$.

Thus, $S$ can occur as a composition factor of an $\ell$-Brauer $m$-rational group for only finitely many pairs $(n, q)$.

Corollary 5.4.5. $\operatorname{PSp}_{2 n}(q)$ can occur as a composition factor of an $\ell$-Brauer rational group if and only if $(n, q) \in\{(2,2),(2,3),(3,2),(4,2)\}$, and $\Omega_{2 n+1}(q)$ cannot occur as a composition factor of any $\ell$-Brauer rational group, where $\ell \nmid q$ in both cases.

Proof. Fix $m=1$. First, as a Zsigmondy prime does not exist for $2^{6}-1$, we must handle the cases $\mathrm{S}_{6}(2)$ and $\mathrm{S}_{12}(2)$. First, we notice that $\mathrm{S}_{6}(2)$ is rational, and hence is $\ell$-Brauer rational for all $\ell>2$. Further, $\operatorname{Out}\left(\mathrm{S}_{12}(2)\right)=1$, and thus, by Corollary 5.1.3. $\mathrm{S}_{12}(2)$ can occur as a composition factor of an $\ell$-Brauer rational group iff $\mathrm{S}_{12}(2)$ is itself $\ell$-Brauer rational. However, applying Lemma 5.1.4, we observe that $\mathrm{S}_{12}(2)$ has ordinary characters $\chi_{1}$ and $\chi_{2}$ that take on irrationalities on the classes of order 63 and 65 , respectively. As $(63,65)=1$, it follows that $\mathrm{S}_{12}(2)$ cannot occur as a composition factor of any $\ell$-Brauer rational group for $\ell>2$.

Now, by Theorem 5.4.1 and Lemma 5.4.2, we must determine when $\frac{\phi(N)}{2 n}|| \operatorname{Out}(S) \mid=\left\{\begin{array}{ll}2(2, q-1) f & q \text { even and } n=2 \\ (2, q-1) f & \text { else }\end{array} \quad\right.$ or $\quad \frac{\phi\left(N^{\prime}\right)}{2 n}|| \operatorname{Out}(S) \mid$

In the notation of the proof of Theorem 5.4.1 (and using the crudest bounds), we determine that $n_{0}=27$, and for each $n<n_{0}$, we obtain the following values $q_{0}$ :

\begin{tabular}{|c|c|c|c|c|c|c|c|c|c|c|c|c|c|c|c|}
\hline$n$ & 2 & 3 & 4 & 5 & 6 & 7 & 8 & 9 & 10 & 11 & 12 & 13 & 14 & 15 & 16 \\
\hline$q_{0}$ & 257 & 398768 & 390 & 47 & 17 & 9 & 7 & 6 & 5 & 4 & 3 & 3 & 3 & 3 & 3 \\
\hline
\end{tabular}

\begin{tabular}{|c|c|c|c|c|c|c|c|c|c|c|}
\hline$n$ & 17 & 18 & 19 & 20 & 21 & 22 & 23 & 24 & 25 & 26 \\
\hline$q_{0}$ & 3 & 3 & 3 & 3 & 3 & 3 & 3 & 3 & 3 & 3 \\
\hline
\end{tabular}

Verifying which of these finitely many pairs satisfy the necessary divisibility relations, we see that the only possible pairs $(n, q)$ lie in the following set:

$$
\{(2,2),(2,3),(2,4),(2,5),(2,7),(2,9),(3,2),(3,3),(4,2),(4,3)\} \text {. }
$$


Using GAP, we see that $\mathrm{S}_{4}(2)$ and $\operatorname{Aut}\left(\mathrm{S}_{4}(3)\right)$ are rational groups, and hence, $\mathrm{S}_{4}(2)^{\prime}$ and $\mathrm{S}_{4}(3)$ occur as a composition factor of an $\ell$-Brauer rational group for all primes $\ell$.

We have that the (non-defining characteristic) primes dividing $\left|\mathrm{S}_{4}(4)\right|$ are 3, 5, and 17. To see that $\mathrm{S}_{4}(4)$ cannot occur as a composition factor of any 3-Brauer, 5-Brauer, 17-Brauer, or $\ell$-Brauer rational group $G$, we follow the argument used for $\mathrm{L}_{2}(7)$. In the case that $N_{G}\left(S_{1}\right) / C_{G}\left(S_{1}\right) \cong S$, choose a liftable irrational character of degree 51 (if our prime is not 5) or a liftable irrational character of degree 225 (if our prime is 5). In the case that $N_{G}\left(S_{1}\right) / C_{G}\left(S_{1}\right) \cong S .2$ or $N_{G}\left(S_{1}\right) / C_{G}\left(S_{1}\right) \cong S .4$, choose a rational character of $S$ of degree 18 that extends to a liftable irrational character of $S .2$ and S.4.

We have that the (non-defining characteristic) primes dividing $\left|\mathrm{S}_{4}(5)\right|$ are 2, 3, and 13. To show that $\mathrm{S}_{4}(5)$ cannot occur as a composition factor of any 2-Brauer, 3-Brauer, or $\ell$-Brauer rational group, where $\ell \nmid\left|S_{4}(5)\right|$, we observe that there is a liftable character $\chi$ of degree 576 that satisfies $3 \mid[\mathbb{Q}(\chi): \mathbb{Q}]$, a clear violation of Theorem 5.1.1, as $\left|\operatorname{Out}\left(\mathrm{S}_{4}(5)\right)\right|=2$. To see that $\mathrm{S}_{4}(5)$ cannot occur as a composition factor of any 13-Brauer rational group $G$, we follow the argument used for $\mathrm{L}_{2}(7)$. In the case that $N_{G}\left(S_{1}\right) / C_{G}\left(S_{1}\right) \cong S$, choose a liftable irrational character of degree 13 . In the case that $N_{G}\left(S_{1}\right) / C_{G}\left(S_{1}\right) \cong S .2$, choose a rational character of $S$ of degree 624 that extends to a liftable irrational character of S.2.

We have that the (non-defining characteristic) primes dividing $\left|\mathrm{S}_{4}(7)\right|$ are 2, 3, and 5. To show that $\mathrm{S}_{4}(7)$ cannot occur as a composition factor of any 2-Brauer, 3-Brauer, or $\ell$-Brauer rational group, where $\ell \nmid\left|S_{4}(7)\right|$, we observe that there is a liftable character $\chi$ of degree 2304 that satisfies $5 \mid[\mathbb{Q}(\chi): \mathbb{Q}]$, a clear violation of Theorem 5.1.1, as $\left|\operatorname{Out}\left(\mathrm{S}_{4}(7)\right)\right|=2$. To see that $\mathrm{S}_{4}(7)$ cannot occur as a composition factor of any 5-Brauer rational group $G$, we observe that there is a character $\chi$ of degree 1200 that satisfies $4 \mid[\mathbb{Q}(\chi): \mathbb{Q}]$, another violation of Theorem 5.1.1.

We have that the (non-defining characteristic) primes dividing $\left|\mathrm{S}_{4}(9)\right|$ are 2, 5, 
and 41. To show that $\mathrm{S}_{4}(9)$ cannot occur as a composition factor of any 2-Brauer, 5-Brauer, or $\ell$-Brauer rational group, where $\ell \nmid\left|S_{4}(9)\right|$, we observe that there is a character $\chi$ of degree 6400 that satisfies $10 \mid[\mathbb{Q}(\chi): \mathbb{Q}]$, a clear violation of Theorem 5.1.1, as $\left|\operatorname{Out}\left(\mathrm{S}_{4}(9)\right)\right|=4$. To see that $\mathrm{S}_{4}(9)$ cannot occur as a composition factor of any 41-Brauer rational group $G$, we observe that there is a character $\chi$ of degree 6560 that satisfies $4 \mid[\mathbb{Q}(\chi): \mathbb{Q}]$ and extends to $\mathrm{S}_{4}(9) .2_{2}$, another violation of Lemma 5.1 .2 .

We have that the (non-defining characteristic) primes dividing $\left|\mathrm{S}_{6}(3)\right|$ are 2, 5, 7, and 13. To show that $\mathrm{S}_{6}(3)$ cannot occur as a composition factor of any 2-Brauer, 5-Brauer, 7-Brauer, or $\ell$-Brauer rational group, where $\ell \nmid\left|S_{6}(3)\right|$, we observe that there is a liftable character $\chi$ of degree 17920 that satisfies $2 \mid[\mathbb{Q}(\chi): \mathbb{Q}]$ and extends to $\mathrm{S}_{6}(3) .2$, a violation of Lemma 5.1.2, as $\left|\operatorname{Out}\left(\mathrm{S}_{6}(3)\right)\right|=2$. To see that $\mathrm{S}_{6}(3)$ cannot occur as a composition factor of any 13-Brauer rational group $G$, we follow the argument used for $\mathrm{L}_{2}(7)$. In the case that $N_{G}\left(S_{1}\right) / C_{G}\left(S_{1}\right) \cong S$, choose a liftable irrational character of degree 13 . In the case that $N_{G}\left(S_{1}\right) / C_{G}\left(S_{1}\right) \cong S .2$, choose a rational character of $S$ of degree 16640 that extends to a liftable irrational character of $S .2$.

We have that the (non-defining characteristic) primes dividing $\left|\mathrm{S}_{8}(2)\right|$ are 3, 5, 7, and 17. To show that $\mathrm{S}_{8}(2)$ cannot occur as a composition factor of any 3-Brauer, 5-Brauer, 7-Brauer or $\ell$-Brauer rational group, where $\ell \nmid\left|S_{8}(2)\right|$, we observe that there is a character $\chi$ of degree 42525 that satisfies $2 \mid[\mathbb{Q}(\chi): \mathbb{Q}]$, a clear violation of Theorem 5.1.1, as $\left|\operatorname{Out}\left(\mathrm{S}_{8}(2)\right)\right|=1$. Further, using GAP, we see that $\mathrm{S}_{8}(2)$ is 17-Brauer rational.

We have that the (non-defining characteristic) primes dividing $\left|\mathrm{S}_{8}(3)\right|$ are 2, 5, 7, 13, and 41. To show that $\mathrm{S}_{8}(3)$ cannot occur as a composition factor of any 2Brauer, 5-Brauer, 7-Brauer, 13-Brauer, or $\ell$-Brauer rational group, where $\ell \nmid\left|S_{8}(3)\right|$, we observe that there is a liftable character $\chi$ of degree 37273600 that satisfies 5 | $[\mathbb{Q}(\chi): \mathbb{Q}]$, a clear violation of Theorem 5.1.1, as $\left|\operatorname{Out}\left(\mathrm{S}_{8}(3)\right)\right|=2$. Likewise, $\mathrm{S}_{8}(3)$ 
cannot occur as a composition factor of any 41-Brauer rational group as there is a liftable character $\chi$ of degree 14694400 that satisfies $4 \mid[\mathbb{Q}(\chi): \mathbb{Q}]$, another violation of Theorem 5.1.1.

We have that the (non-defining characteristic) primes dividing $\left|\mathrm{O}_{7}(3)\right|$ are 2, 5, 7, and 13. To show that $\mathrm{O}_{7}(3)$ cannot occur as a composition factor of any 2-Brauer, 5-Brauer, 7-Brauer, or $\ell$-Brauer rational group, where $\ell \nmid\left|\mathrm{O}_{7}(3)\right|$, we observe that there is a liftable character $\chi$ of degree 17920 that satisfies $2 \mid[\mathbb{Q}(\chi): \mathbb{Q}]$ and extends to $\mathrm{O}_{7}(3) .2$, a violation of Lemma 5.1.2, as $\left|\mathrm{Out}\left(\mathrm{O}_{7}(3)\right)\right|=2$. To see that $\mathrm{O}_{7}(3)$ cannot occur as a composition factor of any 13-Brauer rational group $G$, we follow the argument used for $\mathrm{L}_{2}(7)$. In the case that $N_{G}\left(S_{1}\right) / C_{G}\left(S_{1}\right) \cong S$, choose a liftable irrational character of degree 1560 . In the case that $N_{G}\left(S_{1}\right) / C_{G}\left(S_{1}\right) \cong S .2$, choose a rational character of $S$ of degree 16640 that extends to a liftable irrational character of $S .2$.

We have that the (non-defining characteristic) primes dividing $\left|\mathrm{O}_{9}(3)\right|$ are 2, 5, 7, 13, and 41. To show that $\mathrm{O}_{9}(3)$ cannot occur as a composition factor of any 2Brauer, 5-Brauer, 7-Brauer, 13-Brauer, or $\ell$-Brauer rational group, where $\ell \nmid\left|\mathrm{O}_{9}(3)\right|$, we observe that there is a liftable character $\chi$ of degree 37273600 that satisfies 5 | $[\mathbb{Q}(\chi): \mathbb{Q}]$, a clear violation of Theorem 5.1.1, as $\left|\operatorname{Out}\left(\mathrm{O}_{9}(3)\right)\right|=2$. Likewise, $\mathrm{O}_{9}(3)$ cannot occur as a composition factor of any 41-Brauer rational group as there is a liftable character $\chi$ of degree 14694400 that satisfies $4 \mid[\mathbb{Q}(\chi): \mathbb{Q}]$, another violation of Theorem 5.1.1.

\begin{tabular}{|c|c|c|c|c|}
\hline$(n, q)$ & $(2,2)$ & $(2,3)$ & $(2,4)$ & $(3,2)$ \\
\hline Prime(s) $\ell$ & Any prime $\ell$ & Any prime $\ell$ & Any prime $\ell$ & 17 \\
\hline$\ell$-Brauer Rational Group & $\mathrm{Sp}_{4}(2)$ & Aut $\left(\mathrm{S}_{4}(3)\right)$ & $\mathrm{S}_{6}(2) \cong \mathrm{O}_{7}(2)$ & $\mathrm{S}_{8}(2) \cong \mathrm{O}_{9}(2)$ \\
\hline
\end{tabular}

Note that $\mathrm{Sp}_{4}(2)^{\prime} \cong \mathrm{A}_{6}$ and $\mathrm{S}_{4}(3) \cong \mathrm{U}_{4}(2)$, and thus, we remove $\mathrm{Sp}_{4}(2)^{\prime}$ and $\mathrm{S}_{4}(3)$ from our list in the statement of Theorem 5.0.5. 


\subsection{The Even-Dimensional Orthogonal Groups}

Theorem 5.5.1. Let $S=\mathrm{O}_{2 n}^{+}(q), q=p^{f}, p \neq \ell, n \geq 4$, and $(n, q) \neq(4,2)$. Then $S$ can occur as a composition factor of an $\ell$-Brauer $m$-rational group for only finitely many pairs $(n, q)$.

Proof. Let $L=\operatorname{Spin}_{2 n}^{+}(q)$ so that $L^{*}=\mathrm{PCO}_{2 n}^{+}(q)^{\circ}$. Let $s$ generate a cyclic subgroup of a maximal torus $T^{*} \cong C_{q^{n-1}+1} \times C_{q+1}$ of $L^{*}$ of index $\left(q^{n-1}+1, q+1\right)_{2^{\prime}}\left(q^{n-1}+1\right)_{2}(q+$ $1)_{2}$, [50, Theorem 1]. If $n$ is even, define $s^{\prime}$ to be a generator of a subgroup of index $\left(q^{n-1}-1\right)_{2}(q-1)_{2}\left(q^{n-1}-1, q-1\right)_{2^{\prime}}$ of a maximal torus $T^{* \prime}$ of $L^{*}$ of order $\left(q^{n-1}-1\right)(q-1)$, and if $n$ is odd, define $s^{\prime \prime}$ to be a generator of the unique subgroup of index $\left(q^{n}-1\right)_{2}$ of a maximal torus $T^{* \prime \prime}$ of $L^{*}$ of order $q^{n}-1$, [50]. Let $r$ be a primitive prime divisor of $q^{2(n-1)}-1$, let $r^{\prime}$ be a primitive prime divisor of $q^{n-1}-1$ if $n$ is even, and let $r^{\prime \prime}$ be a primitive prime divisor of $q^{n}-1$ if $n$ is odd. Then, following the proof of Lemma 5.2 .3 , we see that generators of $\langle s\rangle$ lie in $\frac{\phi(N)}{2(n-1)}$ distinct $L^{*}$-conjugacy classes, where $N=\frac{\left(q^{n-1}+1\right)(q+1)}{\left(q^{n-1}+1, q+1\right)_{2^{\prime}}\left(q^{n-1}+1\right)_{2}(q+1)_{2}}$, as $W\left(T^{*}\right) \cong C_{2(n-1)}$, [50, Theorem 1]. Moreover, when $n$ is even, the generators of $\left\langle s^{\prime}\right\rangle$ lie in $\frac{\phi\left(N^{\prime}\right)}{2(n-1)}$ distinct $L^{*}$-conjugacy classes, where $N^{\prime}=\frac{\left(q^{n-1}-1\right)(q-1)}{\left(q^{n-1}-1, q-1\right)_{2^{\prime}}\left(q^{n-1}-1\right)_{2}(q-1)_{2}}$, as $W\left(T^{* \prime}\right) \cong C_{2(n-1)}$, and when $n$ is odd, the generators of $\left\langle s^{\prime \prime}\right\rangle$ lie in $\frac{\phi\left(N^{\prime \prime}\right)}{n}$ distinct $L^{*}$-conjugacy classes, where $N^{\prime \prime}=\frac{q^{n}-1}{\left(q^{n}-1\right)_{2}}$, as $W\left(T^{* \prime \prime}\right) \cong C_{n},\left[50\right.$, Theorem 1]. Note that $\left(N, N^{\prime}\right)=1$ and $\left(N, N^{\prime \prime}\right)=1$, and therefore, $\left[\mathbb{Q}\left(\chi_{s}^{\circ}\right): \mathbb{Q}\right]=\frac{\phi(N)}{2(n-1)}$ when $\ell \nmid|s|,\left[\mathbb{Q}\left(\chi_{s^{\prime}}^{\circ}\right): \mathbb{Q}\right]=\frac{\phi\left(N^{\prime}\right)}{2(n-1)}$ when $n$ is even and $\ell \nmid\left|s^{\prime}\right|$, or $\left[\mathbb{Q}\left(\chi_{s^{\prime \prime}}^{\circ}\right): \mathbb{Q}\right]=\frac{\phi\left(N^{\prime \prime}\right)}{n}$ when $n$ is odd and $\ell \nmid\left|s^{\prime \prime}\right|$.

Using Lemmas 3.1.7 and 5.1.9, we determine that there exists an $n_{0} \in \mathbb{N}$ such that $\frac{\phi(N)}{2(n-1)}>m|\operatorname{Out}(S)|, \frac{\phi\left(N^{\prime}\right)}{2(n-1)}>m|\operatorname{Out}(S)|$, and $\frac{\phi\left(N^{\prime \prime}\right)}{n}>m|\operatorname{Out}(S)|$ for all $n \geq n_{0}$ and $q \geq 2$, where

$$
|\operatorname{Out}(S)|= \begin{cases}6(2, q-1)^{2} f & n=4 \\ 2(2, q-1)^{2} f & n \geq 6, n \text { even } \\ 2\left(4, q^{n}-1\right) f & n \geq 5, n \text { odd }\end{cases}
$$


as provided in [29, Table 5.1.A]:

$$
\begin{gathered}
\frac{\phi(N)}{2(n-1)|\operatorname{Out}(S)|} \geq \sqrt{\frac{q^{n-5}}{4608(n-1)^{2}}} \geq \sqrt{\frac{2^{n-14}}{9(n-1)^{2}}}, \\
\frac{\phi\left(N^{\prime}\right)}{2(n-1)|\operatorname{Out}(S)|} \geq \sqrt{\frac{q^{n-5}}{9216 n^{3}}} \geq \sqrt{\frac{2^{n-15}}{9 n^{3}}}, \\
\frac{\text { and }}{n|\operatorname{Out}(S)|} \geq \sqrt{\frac{q^{n-5}}{2304 n^{3}}} \geq \sqrt{\frac{2^{n-13}}{9 n^{3}}} .
\end{gathered}
$$

Further, for each $4 \leq n \leq n_{0}$, there exists $q_{0} \in \mathbb{N}$ such that $\frac{\phi(N)}{2(n-1)}>m|\operatorname{Out}(S)|$ for all $q \geq q_{0}$ :

$$
\begin{gathered}
\frac{\phi(N)}{2(n-1)|\operatorname{Out}(S)|} \geq \sqrt{\frac{q^{n-3}}{4608(n-1)^{2}\left(\log _{2}(q)\right)^{2}}}, \\
\frac{\text { N } \left.^{\prime}\right)}{2(n-1)|\operatorname{Out}(S)|} \geq \sqrt{\frac{q^{n-3}}{9216 n^{3}\left(\log _{2}(q)\right)^{2}}}, \\
\text { and } \\
\frac{\phi(N)}{2(n-1)|\operatorname{Out}(S)|} \geq \sqrt{\frac{q^{n-2}}{2304 n^{3}\left(\log _{2}(q)\right)^{2}}} .
\end{gathered}
$$

Therefore,

$$
\frac{\phi(N)}{2(n-1)}|m| \operatorname{Out}(S)\left|, \quad \frac{\phi\left(N^{\prime}\right)}{2(n-1)}\right| m|\operatorname{Out}(S)|, \quad \text { or } \quad \frac{\phi\left(N^{\prime \prime}\right)}{n}|m| \operatorname{Out}(S) \mid
$$

for only finitely many pairs $(n, q)$, and $S$ can occur as a composition factor of an $\ell$-Brauer $m$-rational group for only finitely many pairs $(n, q)$.

Corollary 5.5.2. Let $S=\mathrm{O}_{2 n}^{+}(q), q=p^{f}, n \geq 4$, and $p \neq \ell$. Then $S$ can occur as a composition factor of an $\ell$-Brauer rational group if and only if $(n, q) \in\{(4,2),(4,3)\}$.

Proof. Fix $m=1$. As a Zsigmondy prime does not exist for $2^{6}-1$, we first note that $\mathrm{O}_{8}^{+}(2)$ is rational. Now, we must determine which pairs $(n, q)$ satisfy the divisibility relations

$$
\frac{\phi(N)}{2(n-1)}|| \operatorname{Out}(S) \mid= \begin{cases}6(2, q-1)^{2} f & n=4 \\ 2(2, q-1)^{2} f & n \geq 6, n \text { even } \\ 2\left(4, q^{n}-1\right) f & n \geq 5, n \text { odd }\end{cases}
$$




$$
\frac{\phi\left(N^{\prime}\right)}{2(n-1)}|| \operatorname{Out}(S) \mid, \quad \text { or } \quad \frac{\phi\left(N^{\prime \prime}\right)}{n}|| \operatorname{Out}(S) \mid
$$

obtained in the proof of Theorem 5.5.1. In the notation of the proof of Theorem 5.5.1, we see that $n_{0}=34$, and for each $4 \leq n<n_{0}$, we give $q_{0}$ in the following table:

\begin{tabular}{|c|c|c|c|c|c|c|c|c|c|c|c|c|c|c|c|}
\hline$n$ & 4 & 5 & 6 & 7 & 8 & 9 & 10 & 11 & 12 & 13 & 14 & 15 & 16 & 17 & 18 \\
\hline$q_{0}$ & 491715301 & 3157 & 549 & 48 & 43 & 13 & 15 & 7 & 9 & 5 & 6 & 4 & 5 & 3 & 4 \\
\hline
\end{tabular}

\begin{tabular}{|c|c|c|c|c|c|c|c|c|c|c|c|c|c|c|c|}
\hline$n$ & 19 & 20 & 21 & 22 & 23 & 24 & 25 & 26 & 27 & 28 & 29 & 30 & 31 & 32 & 33 \\
\hline$q_{0}$ & 3 & 4 & 3 & 3 & 3 & 3 & 3 & 3 & 3 & 3 & 3 & 3 & 3 & 3 & \\
\hline
\end{tabular}

Verifying which of the (finitely many) remaining pairs $(n, q)$ satisfy the necessary divisibility relation, we see that $S$ can occur as a composition factor of an quadratic rational group only if

$$
(n, q) \in\{(4,2),(4,3),(4,4),(4,5),(4,9),(6,2)\} .
$$

We have that the (non-defining characteristic) primes dividing $\left|\mathrm{O}_{8}^{+}(3)\right|$ are 2,5 , 7, and 13. To show that $\mathrm{O}_{8}^{+}(3)$ cannot occur as a composition factor of any 2-Brauer, 5-Brauer, 7-Brauer, or $\ell$-Brauer rational group, where $\ell \nmid\left|\mathrm{O}_{8}^{+}(3)\right|$, we see that $\mathrm{O}_{8}^{+}(3)$ has a liftable character $\chi$ of degree 716800 which satisfies $2 \mid[\mathbb{Q}(\chi): \mathbb{Q}]$ and extends to $\mathrm{O}_{8}^{+}(3) . S_{4}$, a violation of Lemma 5.1.2, as $\operatorname{Out}\left(\mathrm{O}_{8}^{+}(3)\right) \cong S_{4}$. Further, using GAP we see that $\mathrm{O}_{8}^{+}(3)$ is 13 -Brauer rational.

In the case of $S=\mathrm{O}_{8}^{+}(4), \mathrm{O}_{8}^{+}(5)$, or $\mathrm{O}_{8}^{+}(9)$, we observe that the centralizer of the triality graph automorphism is isomorphic to $G_{2}(q)$ (see, for example, [28, Proposition 1.4.1(ii)]), and therefore, if we consider a semisimple element inside $G_{2}(q)$, the corresponding semisimple character will be invariant under the triality automorphism. Further, the semisimple characters (as they may be viewed as characters of $\left.\operatorname{Spin}_{8}^{+}(q)\right)$ are invariant under the outer diagonal automorphisms of $O_{8}^{+}(q)$. Therefore, in the case that our chosen element is an element of $G_{2}(q)$, the $R$-orbit length 
of the character can at most be 4, 2, and 4, respectively. However, the semisimple elements chosen above of orders $\frac{q^{3}-1}{\left(q^{3}-1\right)_{2}}$ and $\frac{q^{3}+1}{\left(q^{3}+1\right)_{2}}$ inside maximal tori of $\operatorname{Spin}_{8}^{+}(q)$ of order $\left(q^{3}-1\right)(q-1)$ and $\left(q^{3}+1\right)(q+1)$, respectively, are elements of $G_{2}(q)$, and these yield characters $\alpha$ satisfying $6=\frac{\phi(63)}{6} \mid[\mathbb{Q}(\alpha): \mathbb{Q}]$ or $8=\frac{\phi(65)}{6} \mid[\mathbb{Q}(\alpha): \mathbb{Q}]$, $5=\frac{\phi(31)}{6} \mid[\mathbb{Q}(\alpha): \mathbb{Q}]$ or $6=\frac{\phi(63)}{6} \mid[\mathbb{Q}(\alpha): \mathbb{Q}]$, and $12=\frac{\phi(91)}{6} \mid[\mathbb{Q}(\alpha): \mathbb{Q}]$ or $48=\frac{\phi(365)}{6} \mid[\mathbb{Q}(\alpha): \mathbb{Q}]$, respectively, each a contradiction of Theorem 5.1.1. Therefore, $\mathrm{O}_{8}^{+}(4), \mathrm{O}_{8}^{+}(5)$, and $\mathrm{O}_{8}^{+}(9)$ cannot occur as composition factors of any $\ell$-Brauer rational group for non-defining characteristic primes $\ell$.

Finally, to show that $\mathrm{O}_{12}^{+}(2)$ cannot occur as a composition factor of any $\ell$ Brauer rational group for $\ell \neq 2$, we observe that $\operatorname{Spin}_{12}^{+}(2)$ has cyclic maximal tori of (coprime) orders $\left(2^{6-1}-1\right)(2-1)=31$ and $\left(2^{6}-1\right)=63$. Choosing generators $s$ and $s^{\prime}$ of the (isomorphic) dual tori in $\left(\operatorname{Spin}_{12}^{+}(2)\right)^{*}$, we obtain ordinary semisimple characters $\chi_{s}$ and $\chi_{s^{\prime}}$ of $\mathrm{O}_{12}^{+}(2)$, respectively. As $\left(|s|,\left|s^{\prime}\right|\right)=1$, the restriction of one of these characters to the $\ell$-regular classes is an irreducible $\ell$-Brauer character. However, we see that both satisfy $3=\frac{\phi(31)}{2(6-1)}=\frac{\phi(63)}{2 \cdot 6}$, and thus, $3 \mid\left[\mathbb{Q}\left(\chi_{s}^{\circ}\right): \mathbb{Q}\right]$ or $3 \mid\left[\mathbb{Q}\left(\chi_{s^{\prime}}^{\circ}\right): \mathbb{Q}\right]$, a violation of Theorem 5.1.1, as $\left|\operatorname{Out}\left(\mathrm{O}_{12}^{+}(2)\right)\right|=2$. Therefore, $\mathrm{O}_{12}^{+}(2)$ cannot occur as a composition factor of any $\ell$-Brauer rational group for $\ell \neq 2$.

\begin{tabular}{|c|c|c|}
\hline Group & $\mathrm{O}_{8}^{+}(2)$ & $\mathrm{O}_{8}^{+}(3)$ \\
\hline Prime(s) $\ell$ & Any prime $\ell$ & 13 \\
\hline$\ell$-Brauer Rational Group & $\mathrm{O}_{8}^{+}(2)$ & $\mathrm{O}_{8}^{+}(3)$ \\
\hline
\end{tabular}

Theorem 5.5.3. Let $S=\mathrm{O}_{2 n}^{-}(q), q^{2}=p^{f}, p \neq \ell, n \geq 4$, and $(n, q) \neq(4,2)$. Then $S$ can occur as a composition factor of an $\ell$-Brauer $m$-rational group for only finitely many pairs $(n, q)$.

Proof. Let $L=\operatorname{Spin}_{2 n}^{-}(q)$ so that $L^{*}=\operatorname{PCO}_{2 n}^{-}(q)^{\circ}$. Let $s$ generate the unique subgroup of a cyclic maximal torus $T^{*} \cong C_{q^{n}+1}$ in $L^{*}$ of index $\left(q^{n}+1\right)_{2}$, and $s^{\prime}$ 
generate the unique subgroup of a maximal torus $T^{* \prime}$ of order $\left(q^{n-1}+1\right)(q-1)$ in $L^{*}$ of index $\left(q^{n-1}+1, q-1\right)_{2^{\prime}}\left(q^{n-1}+1\right)_{2}(q-1)_{2}$, [50, Theorem 1]. Let $r$ be a primitive prime divisor of $q^{2 n}-1$ and $r^{\prime}$ be a primitive prime divisor of $q^{2(n-1)}-1$. Following the proof of Lemma 5.2.3, we see that generators of $\langle s\rangle$ lie in $\frac{\phi(N)}{n} L^{*}$-conjugacy classes, where $N=\frac{q^{n}+1}{\left(q^{n}+1\right)_{2}}$, as $W\left(T^{*}\right) \cong C_{n}$ is cyclic of order $n$. Likewise, the generators of $\left\langle s^{\prime}\right\rangle$ lie in $\frac{\phi\left(N^{\prime}\right)}{2(n-1)} L^{*}$-conjugacy classes, where $N^{\prime}=\frac{\left(q^{n-1}+1\right)(q-1)}{\left(q^{n-1}+1, q-1\right)_{2^{\prime}}\left(q^{n-1}+1\right)_{2}(q-1)_{2}}$, as $W\left(T^{* \prime}\right) \cong C_{2(n-1)}$ is cyclic of order $2(n-1)$ [50, Theorem 1]. Note that $\left(N, N^{\prime}\right)=1$ by Lemma 5.1.8. Hence, $\left[\mathbb{Q}\left(\chi_{s}^{\circ}\right): \mathbb{Q}\right]=\frac{\phi(N)}{n}$ if $\ell \nmid|s|$ or $\left[\mathbb{Q}\left(\chi_{s^{\prime}}^{\circ}\right): \mathbb{Q}\right]=\frac{\phi\left(N^{\prime}\right)}{2(n-1)}$ if $\ell \nmid\left|s^{\prime}\right|$.

Now, using Lemmas 3.1.7 and 5.1.9, it is easy to see that there exists $n_{0} \in \mathbb{N}$ such that $\frac{\phi(N)}{n}>m|\operatorname{Out}(S)|=m\left(4, q^{n}+1\right) f$ and $\frac{\phi\left(N^{\prime}\right)}{2(n-1)}>m|\operatorname{Out}(S)|$ for all $n \geq n_{0}$ and $q \geq 2$, where $|\operatorname{Out}(S)|$ is taken from [29, Table 5.1.A]:

$$
\begin{gathered}
\frac{\phi(N)}{\left(4, q^{n}+1\right) f n} \geq \sqrt{\frac{q^{n}+1}{64 q^{2} n^{2}\left(q^{n}+1\right)_{2}}} \geq \sqrt{\frac{2^{n-11}}{n^{2}}} \\
\frac{\phi\left(N^{\prime}\right)}{2(n-1)\left(4, q^{n}+1\right) f} \geq \sqrt{\frac{q^{n-1}+1}{256 q^{2} n^{2}\left(q^{n}+1\right)_{2}}} \geq \sqrt{\frac{2^{n-13}}{n^{2}}}
\end{gathered}
$$

Further, for each $4 \leq n<n_{0}$, there exists $q_{0}$ such that $\frac{\phi(N)}{n}>m\left(4, q^{n}+1\right) f$ and $\frac{\phi\left(N^{\prime}\right)}{2(n-1)}>m\left(4, q^{n}+1\right) f$ for all $q \geq q_{0}$ :

$$
\begin{gathered}
\frac{\phi\left(\frac{q^{n}+1}{\left(q^{n}+1\right)_{2}}\right)}{\left(4, q^{n}+1\right) f n} \geq \sqrt{\frac{q^{n}+1}{128\left(\log _{2}(q)\right)^{2} n^{2}\left(q^{n}+1\right)_{2}}} \geq \sqrt{\frac{q^{n-2}}{128\left(\log _{2}(q)\right)^{2} n^{2}}} \\
\frac{\phi\left(\frac{\text { and }}{\left(q^{n-1}+1, q-1\right)_{2^{\prime}}\left(q^{n-1}+1\right)_{2}(q-1)_{2}}\right)}{\left(4, q^{n}+1\right) f n} \geq \sqrt{\frac{q^{n-1}+1}{256\left(\log _{2}(q)\right)^{2} n^{2}\left(q^{n-1}+1\right)_{2}}} \geq \sqrt{\frac{q^{n-1}+1}{256(q+1)\left(\log _{2}(q)\right)^{2} n^{2}}} .
\end{gathered}
$$

Therefore, only finitely many pairs $(n, q)$ can satisfy $\frac{\phi(N)}{n} \mid m\left(4, q^{n}+1\right) f$ or $\frac{\phi\left(N^{\prime}\right)}{2(n-1)} \mid m\left(4, q^{n}+1\right) f$, and in particular, $S$ can occur as a composition factor of an $\ell$-Brauer $m$-rational group for only finitely pairs $(n, q)$. 
Corollary 5.5.4. Let $S=\mathrm{O}_{2 n}^{-}(q), q^{2}=p^{f}, p \neq \ell$, and $n \geq 4$. Then $S$ can occur as a composition factor of a quadratic rational group if and only if $(n, q) \in\{(4,2),(5,2)\}$.

Proof. Fix $m=1$. As a Zsigmondy prime does not exist for $2^{6}-1$, we first handle the case $\mathrm{O}_{8}^{-}(2)$. We have that the (non-defining characteristic) primes dividing $\left|\mathrm{O}_{8}^{-}(2)\right|$ are $3,5,7$, and 17 . To show that $\mathrm{O}_{8}^{-}(2)$ cannot occur as a composition factor of any 3-Brauer, 5-Brauer, 7-Brauer, or $\ell$-Brauer rational group, where $\ell \nmid\left|\mathrm{O}_{8}^{-}(2)\right|$, we see that $\mathrm{O}_{8}^{-}(2)$ has a liftable character $\chi$ of degree 2835 which satisfies $4 \mid[\mathbb{Q}(\chi): \mathbb{Q}]$, a violation of Theorem 5.1.1, as $\left|\operatorname{Out}\left(\mathrm{O}_{8}^{-}(2)\right)\right|=2$. Further, using GAP we see that $\mathrm{O}_{8}^{-}(2) .2$ is 17 -Brauer rational.

Now, from Theorem 5.5.3 we must determine when $\frac{\phi(N)}{n} \mid\left(4, q^{n}+1\right) f$ or $\frac{\phi\left(N^{\prime}\right)}{2(n-1)} \mid\left(4, q^{n}+1\right) f$. In the notation of the proof of Theorem 5.5.3, we calculate that $n_{0}=22$, and for each $4 \leq n<n_{0}, q_{0}$ is given in the following table:

\begin{tabular}{|c|c|c|c|c|c|c|c|c|c|}
\hline$n$ & 4 & 5 & 6 & 7 & 8 & 9 & 10 & 11 & 12 \\
\hline$q_{0}$ & 590 & 62 & 21 & 12 & 8 & 6 & 5 & 4 & 4 \\
\hline
\end{tabular}

\begin{tabular}{|c|c|c|c|c|c|c|c|c|c|}
\hline$n$ & 13 & 14 & 15 & 16 & 17 & 18 & 19 & 20 & 21 \\
\hline$q_{0}$ & 3 & 3 & 3 & 3 & 3 & 3 & 3 & 3 & 3 \\
\hline
\end{tabular}

Verifying which of these finitely many pairs $(n, q)$ satsify the necessary divisibility condition, we see that $S$ can occur as a composition factor of a quadratic rational group only if $(n, q) \in\{(4,2),(4,3),(5,2),(6,2)\}$.

We have that the (non-defining characteristic) primes dividing $\left|\mathrm{O}_{8}^{-}(3)\right|$ are $2,5,7$, 13, and 41. To show that $\mathrm{O}_{8}^{-}(3)$ cannot occur as a composition factor of any 2-Brauer, 5-Brauer, 7-Brauer, 13-Brauer, or $\ell$-Brauer rational group, where $\ell \nmid\left|\mathrm{O}_{8}^{-}(3)\right|$, we see that $\mathrm{O}_{8}^{-}(3)$ has a liftable character $\chi$ of degree 465920 which satisfies $10 \mid[\mathbb{Q}(\chi): \mathbb{Q}]$, a violation of Theorem 5.1.1, as $\left|\operatorname{Out}\left(\mathrm{O}_{8}^{-}(3)\right)\right|=4$. To show that $\mathrm{O}_{8}^{-}(3)$ cannot occur as a composition factor of any 41-Brauer rational group, we see that $\mathrm{O}_{8}^{-}(3)$ has a 
liftable character $\chi$ of degree 367360 which satisfies $4 \mid[\mathbb{Q}(\chi): \mathbb{Q}]$ and extends to $\mathrm{O}_{8}^{-}(3) .2_{2}$, another violation of Lemma 5.1.2.

We have that the (non-defining characteristic) primes dividing $\left|\mathrm{O}_{10}^{-}(2)\right|$ are 3 , $5,7,11$, and 17 . To show that $\mathrm{O}_{10}^{-}(2)$ cannot occur as a composition factor of any 5-Brauer, 7-Brauer, 17-Brauer, or $\ell$-Brauer rational group, where $\ell \nmid\left|\mathrm{O}_{10}^{-}(2)\right|$, we see that $\mathrm{O}_{10}^{-}(2)$ has a liftable character $\chi$ of degree 722925 which satisfies $4 \mid[\mathbb{Q}(\chi): \mathbb{Q}]$, a violation of Theorem 5.1.1, as $\left|\operatorname{Out}\left(\mathrm{O}_{10}^{-}(2)\right)\right|=2$. To show that $\mathrm{O}_{10}^{-}(2)$ cannot occur as a composition factor of any 3-Brauer or 11-Brauer rational group, we see that $\mathrm{O}_{10}^{-}(2)$ has a liftable character $\chi$ of degree 1403325 which satisfies $2 \mid[\mathbb{Q}(\chi): \mathbb{Q}]$ and extends to $\mathrm{O}_{10}^{-}(2) .2$, another violation of Lemma 5.1 .2 .

We have that the (non-defining characteristic) primes dividing $\left|\mathrm{O}_{12}^{-}(2)\right|$ are 3,5 , $7,11,13,17$, and 31 . To show that $\mathrm{O}_{12}^{-}(2)$ cannot occur as a composition factor of any 3-Brauer, 7-Brauer, 11-Brauer, 17-Brauer, 31-Brauer, or $\ell$-Brauer rational group, where $\ell \nmid\left|\mathrm{O}_{12}^{-}(2)\right|$, we consider the semisimple character $\chi_{s}$ labelled by the element $s \in\left(\operatorname{Spin}_{12}^{-}(2)\right)^{*}$ of order $2^{6}+1$. This yields $4=\frac{\phi(65)}{2 \cdot 6} \mid\left[\mathbb{Q}\left(\chi_{s}^{\circ}\right): \mathbb{Q}\right]$ by Lemma 5.2 .4 . a violation of Theorem 5.1.1, as $\left|\operatorname{Out}\left(\mathrm{O}_{12}^{-}(2)\right)\right|=2$. Likewise, to show that $\mathrm{O}_{12}^{-}(2)$ cannot occur as a composition factor of any 5-Brauer or 13-Brauer rational group, we consider the semisimple character $\chi_{s}$ labelled by the element $s \in\left(\operatorname{Spin}_{12}^{-}(2)\right)^{*}$ of order $2^{6}-1$. This yields $3=\frac{\phi\left(2^{6}-1\right)}{2 \cdot 6} \mid\left[\mathbb{Q}\left(\chi_{s}^{\circ}\right): \mathbb{Q}\right]$ by Lemma 5.2.4, another violation of Theorem 5.1.1.

\begin{tabular}{|c|c|}
\hline Group & $\mathrm{O}_{8}^{-}(2)$ \\
\hline Prime(s) $\ell$ & 17 \\
\hline Ł-Brauer Rational Group & $\mathrm{O}_{8}^{-}(2) .2$ \\
\hline
\end{tabular}




\subsection{The Exceptional Groups}

Let $\mathcal{L}$ be a simple simply connected algebraic group and $F$ a Frobenius map on $\mathcal{L}$ so that $L:=\mathcal{L}^{F}$ and $S=L / Z(L)$ is a simple exceptional group of Lie type. In all cases, we choose a regular semisimple $\ell^{\prime}$-element $s \in\left[L^{*}, L^{*}\right]$ with connected centralizer to construct $\chi_{s}^{\circ} \in \operatorname{IBr}_{\ell}(S)$. Then, selecting a primitive prime divisor $r$ and calculating the number of conjugacy classes that generators of $\langle s\rangle$ lie in, we obtain a divisibility relation that must be satisfied in order for $S$ to be realizable as a composition factor of an $\ell$-Brauer $m$-rational group. In the following table, we give two cyclic maximal tori $T^{*}$ and $T^{* \prime}$, as well as the corresponding generators, $s$ and $s^{\prime}$ (of coprime order), of particular subgroups of the tori, [27]:

\begin{tabular}{|c|c|c|}
\hline Group & Cyclic Maximal Torus $T^{*}$ Order & Cyclic Maximal Torus $T^{* \prime}$ Order \\
\hline$E_{6}(q), q=p^{f}$ & $q^{6}+q^{3}+1$ & $\left(q^{4}-q^{2}+1\right)\left(q^{2}+q+1\right)$ \\
\hline$E_{7}(q), q=p^{f}$ & $\left(q^{6}-q^{3}+1\right)(q+1)$ & $q^{7}+1$ \\
\hline$E_{8}(q), q=p^{f}$ & $q^{8}+q^{7}-q^{5}-q^{4}-q^{3}+q+1$ & $q^{8}-q^{7}+q^{5}-q^{4}+q^{3}-q+1$ \\
\hline$F_{4}(q), q=p^{f}$ & $q^{4}-q^{2}+1$ & $q^{4}+1$ \\
\hline$G_{2}(q), q=p^{f}$ & $q^{2}-q+1$ & $q^{2}+q+1$ \\
\hline${ }^{2} B_{2}(q), q=2^{f}$ & $q+\sqrt{2 q}+1$ & $\left(q^{3}+1\right)(q-1)$ \\
\hline${ }^{3} D_{4}(q), q^{3}=p^{f}$ & $q^{4}-q^{2}+1$ & $\left(q^{4}-q^{2}+1\right)\left(q^{2}-q+1\right)$ \\
\hline${ }^{2} E_{6}(q), q^{2}=p^{f}$ & $q^{6}-q^{3}+1$ & $q^{2}+q+1-\left(\sqrt{2 q^{3}}+\sqrt{2 q}\right)$ \\
\hline${ }^{2} F_{4}(q), q=2^{f}$ & $q^{2}+q+1+\left(\sqrt{2 q^{3}}+\sqrt{2 q}\right)$ & $q-\sqrt{3 q}+1$ \\
\hline${ }^{2} G_{2}(q), q=3^{f}$ & $q+\sqrt{3 q}+1$ &
\end{tabular}

In all cases, we may choose the primitive prime divisors listed below. Following the proof of Lemma 5.2.3, noting that $\left(s^{\prime}\right)^{\frac{\left|s^{\prime}\right|}{r^{\prime}}}$ and $s^{\frac{|s|}{r}}$ are regular elements, [36, Lemma 2.3], we obtain the divisibility relations stated below (where $|\operatorname{Out}(S)|$ can be found in [29, Table 5.1.B]). Finally, note that the cases ${ }^{3} D_{4}(2)$ and $G_{2}(2)^{\prime}$ are handled separately when discussing $\ell$-Brauer rationality, as a primitive prime divisor of $2^{6}-1$ does not exist. 


\begin{tabular}{|c|c|c|}
\hline Group & Element $s$ Order & Element $s^{\prime}$ order \\
\hline$E_{6}(q), q=p^{f}$ & $\frac{q^{6}+q^{3}+1}{(3, q-1)}$ & $\frac{\left(q^{4}-q^{2}+1\right)\left(q^{2}+q+1\right)}{(3, q-1)}$ \\
\hline$E_{7}(q), q=p^{f}$ & $\frac{\left(q^{6}-q^{3}+1\right)(q+1)}{q+1}$ & $\frac{q^{7}+1}{q+1}$ \\
\hline$E_{8}(q), q=p^{f}$ & $q^{8}+q^{7}-q^{5}-q^{4}-q^{3}+q+1$ & $q^{8}-q^{7}+q^{5}-q^{4}+q^{3}-q+1$ \\
\hline$F_{4}(q), q=p^{f}$ & $q^{4}-q^{2}+1$ & $q^{4}+1$ \\
\hline$G_{2}(q), q=p^{f}$ & $q^{2}-q+1$ & $q^{2}+q+1$ \\
\hline${ }^{2} B_{2}(q), q=2^{f}$ & $q+\sqrt{2 q}+1$ & $\left(q^{3}+1\right)(q-1)$ \\
\hline${ }^{3} D_{4}(q), q^{3}=p^{f}$ & $q^{4}-q^{2}+1$ & $\frac{\left(q^{4}-q^{2}+1\right)\left(q^{2}-q+1\right)}{(3, q+1)}$ \\
\hline${ }^{2} E_{6}(q), q^{2}=p^{f}$ & $\frac{q^{6}-q^{3}+1}{(3, q+1)}$ & $q-\sqrt{3 q}+1$ \\
\hline${ }^{2} F_{4}(q), q=2^{f}$ & $q^{2}+q+1+\left(\sqrt{2 q^{3}}+\sqrt{2 q}\right)$ & $q^{2}+q+1-\left(\sqrt{2 q^{3}}+\sqrt{2 q}\right)$ \\
\hline${ }^{2} G_{2}(q), q=3^{f}$ & $q+\sqrt{3 q}+1$ & $q$ \\
\hline
\end{tabular}

\begin{tabular}{|c|c|c|c|}
\hline Group & $r$ a P.P.D. of & $r^{\prime}$ a P.P.D. of & Divisibility Relations \\
\hline$E_{6}(q), q=p^{f}$ & $q^{9}-1$ & $q^{12}-1$ & $\frac{\phi(|s|)}{9}$ or $\frac{\phi\left(\left|s^{\prime}\right|\right)}{12} \mid 2 m(3, q-1) f$ \\
\hline$E_{7}(q), q=p^{f}$ & $q^{18}-1$ & $q^{14}-1$ & $\frac{\phi(|s|)}{18}$ or $\frac{\phi\left(\left|s^{\prime}\right|\right)}{14} \mid m(2, q-1) f$ \\
\hline$E_{8}(q), q=p^{f}$ & $q^{30}-1$ & $q^{15}-1$ & $\frac{\phi(|s|)}{30}$ or $\frac{\phi\left(\mid s^{\prime}\right)}{30} \mid m f$ \\
\hline$F_{4}(q), q=p^{f}$ & $q^{12}-1$ & $q^{8}-1$ & $\frac{\phi(|s|)}{12}$ or $\frac{\phi\left(\left|s^{\prime}\right|\right)}{8} \mid m(2, q) f$ \\
\hline$G_{2}(q), q=p^{f}$ & $q^{6}-1$ & $q^{3}-1$ & $\frac{\phi(|s|)}{6}$ or $\frac{\phi\left(\left|s^{\prime}\right|\right)}{6} \mid 2 m f$ \\
\hline${ }^{2} B_{2}(q), q=2^{f}$ & $q^{4}-1$ & $q^{4}-1$ & $\frac{\phi(|s|)}{4}$ or $\frac{\phi\left(s^{\prime} \mid\right)}{4} \mid m f$ \\
\hline${ }^{3} D_{4}(q), q^{3}=p^{f}$ & $q^{12}-1$ & $q^{6}-1$ & $\frac{\phi(|s|)}{4}$ or $\frac{\phi\left(\left|s^{\prime}\right|\right)}{4} \mid m f$ \\
\hline${ }^{2} E_{6}(q), q^{2}=p^{f}$ & $q^{18}-1$ & $q^{12}-1$ & $\frac{\phi(|s|)}{9}$ or $\frac{\phi\left(\left|s^{\prime}\right|\right)}{12} \mid m(3, q+1) f$ \\
\hline${ }^{2} F_{4}(q), q=2^{f}$ & $q^{12}-1$ & $q^{12}-1$ & $\frac{\phi(|s|)}{12}$ or $\frac{\phi\left(\left|s^{\prime}\right|\right)}{12} \mid m f$ \\
\hline${ }^{2} G_{2}(q), q=3^{f}$ & $q^{6}-1$ & $q^{6}-1$ & $\frac{\phi(s)}{6}$ or $\frac{\phi\left(s^{\prime} \mid\right)}{6} \mid m f$ \\
\hline
\end{tabular}

Using the divisbility relations stated in the above table, it is clear from Lemma 3.1.7 that we have the following theorem:

Theorem 5.6.1. Only finitely many simple exceptional groups of Lie type in characteristic $p \neq \ell$ can occur as a composition factor of some $\ell$-Brauer $m$-rational group.

Corollary 5.6.2. Suppose $S$ is an exceptional group of Lie type in characteristic $p \neq \ell$. Then $S$ can occur as a composition factor of some $\ell$-Brauer rational group if and only if

$$
S \in\left\{\mathrm{F}_{4}(2), \mathrm{G}_{2}(2)^{\prime}, \mathrm{G}_{2}(3), \mathrm{G}_{2}(4),{ }^{3} \mathrm{D}_{4}(2),{ }^{2} \mathrm{E}_{6}(2),{ }^{2} \mathrm{~F}_{4}(2)^{\prime},{ }^{2} \mathrm{G}_{2}(3)^{\prime}\right\} .
$$


Proof. Fix $m=1$. Solving the divisibility relations in the above table, we see that $S$ can occur as a composition factor of an $\ell$-Brauer rational group only if

$$
\begin{gathered}
S \in\left\{\mathrm{F}_{4}(2), \mathrm{G}_{2}(2)^{\prime}, \mathrm{G}_{2}(3), \mathrm{G}_{2}(4), \mathrm{G}_{2}(5),{ }^{2} \mathrm{~B}_{2}(8),{ }^{2} \mathrm{~B}_{2}(32),\right. \\
\left.{ }^{3} \mathrm{D}_{4}(2),{ }^{2} \mathrm{E}_{6}(2),{ }^{2} \mathrm{~F}_{4}(2)^{\prime},{ }^{2} \mathrm{~F}_{4}(8),{ }^{2} \mathrm{G}_{2}(3)^{\prime},{ }^{2} \mathrm{G}_{2}(27)\right\} .
\end{gathered}
$$

Note that $\mathrm{G}_{2}(2)^{\prime} \cong \mathrm{U}_{3}(3)$, and therefore, we must only verify the result for the prime 3. Using GAP, we see that $\mathrm{G}_{2}(2)$ is 3 -Brauer rational.

We have that the (non-defining characteristic) prime divisors of $\left|\mathrm{G}_{2}(3)\right|$ are 2, 7, and 13. To show that $\mathrm{G}_{2}(3)$ cannot occur as a composition factor of any 2-Brauer, 7-Brauer, 13-Brauer, or $\ell$-Brauer rational group, where $\ell \nmid\left|\mathrm{G}_{2}(3)\right|$, observe that $\mathrm{G}_{2}(3)$ has a liftable character $\chi$ of degree 64 which satisfies $2 \mid[\mathbb{Q}(\chi): \mathbb{Q}]$ and extends to $\mathrm{G}_{2}(3) .2$, a violation of Lemma 5.1.2, as $\left|\operatorname{Out}\left(\mathrm{G}_{2}(3)\right)\right|=2$.

We have that the (non-defining characteristic) prime divisors of $\left|\mathrm{G}_{2}(4)\right|$ are 3, 5, 7 , and 13 . To show that $\mathrm{G}_{2}(4)$ cannot occur as a composition factor of any 5 -Brauer, 7-Brauer, 13-Brauer, or $\ell$-Brauer rational group, where $\ell \nmid\left|\mathrm{G}_{2}(4)\right|$, observe that $\mathrm{G}_{2}(4)$ has a liftable character $\chi$ of degree 300 which satisfies $2 \mid[\mathbb{Q}(\chi): \mathbb{Q}]$ and extends to $\mathrm{G}_{2}(4) .2$, a violation of Lemma 5.1.2, as $\left|\operatorname{Out}\left(\mathrm{G}_{2}(4)\right)\right|=2$. To show that $\mathrm{G}_{2}(4)$ cannot occur as a composition factor of any 5-Brauer rational group, we follow the argument presented for $\mathrm{L}_{2}(7)$. In the case that $N_{G}\left(S_{1}\right) / C_{G}\left(S_{1}\right) \cong S$, choose an irrational liftable character of degree 819. In the case that $N_{G}\left(S_{1}\right) / C_{G}\left(S_{1}\right) \cong S .2$, choose a rational character of $S$ of degree 378 that extends to an irrational liftable character of S.2.

We have that the (non-defining characteristic) prime divisors of $\left|\mathrm{G}_{2}(5)\right|$ are 2, 3,7 , and 31 . To show that $\mathrm{G}_{2}(5)$ cannot occur as a composition factor of any 2Brauer, 3-Brauer, 7-Brauer, or $\ell$-Brauer rational group, where $\ell \nmid\left|\mathrm{G}_{2}(5)\right|$, observe that $\mathrm{G}_{2}(5)$ has a liftable character $\chi$ of degree 12096 which satisfies $4 \mid[\mathbb{Q}(\chi): \mathbb{Q}]$, a violation of Theorem 5.1.1, as $\left|\operatorname{Out}\left(\mathrm{G}_{2}(5)\right)\right|=1$. To show that $\mathrm{G}_{2}(5)$ cannot occur as a composition factor of any 31-Brauer rational group, observe that $\mathrm{G}_{2}(5)$ has a 
liftable character $\chi$ of degree 960 which satisfies $2 \mid[\mathbb{Q}(\chi): \mathbb{Q}]$, another violation of Theorem 5.1.1.

We have that the (non-defining characteristic) prime divisors of $|\mathrm{Sz}(8)|$ are 5, 7, and 13. To show that $\mathrm{Sz}(8)$ cannot occur as a composition factor of any 5-Brauer, 7-Brauer, 13-Brauer, or $\ell$-Brauer rational group, where $\ell \nmid|\mathrm{Sz}(8)|$, observe that $\mathrm{Sz}(8)$ has a liftable irrational character $\chi$ of degree 14 which extends to a liftable character of $\mathrm{Sz}(8) .3$, a violation of Lemma 5.1.2, as $|\mathrm{Out}(\mathrm{Sz}(8))|=3$.

We have that the (non-defining characteristic) prime divisors of $|\mathrm{Sz}(32)|$ are 5, 31, and 41. To show that $\mathrm{Sz}(32)$ cannot occur as a composition factor of any 5Brauer, 31-Brauer, $\ell$-Brauer rational group, where $\ell \nmid|\mathrm{Sz}(32)|$, observe that $\mathrm{Sz}(32)$ has a liftable character $\chi$ of degree 775 which satisfies $10 \mid[\mathbb{Q}(\chi): \mathbb{Q}]$, a violation of Theorem 5.1.1, as $|\operatorname{Out}(\mathrm{Sz}(32))|=5$. Likewise, $\mathrm{Sz}(32)$ cannot occur as a composition factor of any 41-Brauer rational group as it contains a liftable character of degree 1025 satisfying $15 \mid[\mathbb{Q}(\chi): \mathbb{Q}]$, another violation of Theorem 5.1.1.

We have that the (non-defining characteristic) prime divisors of $\left|{ }^{3} \mathrm{D}_{4}(2)\right|$ are 3,7 , and 13. Using GAP, we see that ${ }^{3} \mathrm{D}_{4}(2) .3$ is 3 -Brauer rational. To show that ${ }^{3} \mathrm{D}_{4}(2)$ cannot occur as a composition factor of any 7-Brauer, 13-Brauer, or $\ell$-Brauer rational group where $\ell \nmid\left|{ }^{3} \mathrm{D}_{4}(2)\right|$, we follow the argument presented for $\mathrm{L}_{2}(7)$. In the case that $N_{G}\left(S_{1}\right) / C_{G}\left(S_{1}\right) \cong S$, choose an irrational liftable character of degree 1911 . In the case that $N_{G}\left(S_{1}\right) / C_{G}\left(S_{1}\right) \cong S .3$, choose a rational character of $S$ of degree 26 that extends to an irrational liftable character of S.3.

We have that the (non-defining characteristic) prime divisors of $\left|{ }^{2} \mathrm{E}_{6}(2)\right|$ are 3 , $5,7,11,13,17$, and 19 . To show that ${ }^{2} \mathrm{E}_{6}(2)$ cannot occur as a composition factor of any 3-Brauer, 5-Brauer, 7-Brauer, 11-Brauer, 13-Brauer, 19-Brauer, or $\ell$-Brauer rational group where $\ell \nmid\left|{ }^{2} \mathrm{E}_{6}(2)\right|$, we observe that ${ }^{2} \mathrm{E}_{6}(2)$ has a liftable irrational character of degree 65511420975 that extends to ${ }^{2} \mathrm{E}_{6}(2) . S_{3}$, a violation of Lemma 5.1 .2 as $\left|\operatorname{Out}\left({ }^{2} \mathrm{E}_{6}(2)\right)\right|=6$. To show that ${ }^{2} \mathrm{E}_{6}(2)$ cannot occur as a composition factor of any 17-Brauer rational group, we observe that ${ }^{2} \mathrm{E}_{6}(2)$ has a liftable character $\chi$ of 
degree 145411200 that extends to ${ }^{2} \mathrm{E}_{6}(2) .2$ and satisfies $2 \mid[\mathbb{Q}(\chi): \mathbb{Q}]$, a violation of Lemma 5.1 .2 as $\left.\mid \operatorname{Out}^{2} \mathrm{E}_{6}(2)\right) \mid=6$.

We have that the (non-defining characteristic) prime divisors of $\left|{ }^{2} \mathrm{~F}_{4}(2)^{\prime}\right|$ are 3, 5, and 13. To show that ${ }^{2} \mathrm{~F}_{4}(2)^{\prime}$ cannot occur as a composition factor of any 3-Brauer, 5-Brauer, 13-Brauer, or $\ell$-Brauer rational group where $\ell \nmid\left|{ }^{2} \mathrm{~F}_{4}(2)^{\prime}\right|$, we observe that ${ }^{2} \mathrm{~F}_{4}(2)^{\prime}$ has a liftable irrational character of degree 27 that extends to ${ }^{2} \mathrm{~F}_{4}(2)$, a violation of Lemma 5.1 .2 as $\left|\operatorname{Out}\left({ }^{2} \mathrm{~F}_{4}(2)\right)\right|=2$.

We have that the (non-defining characteristic) prime divisors of $\left|{ }^{2} \mathrm{~F}_{4}(8)\right|$ are 3 , $5,7,13,19,37$, and 109 . To show that ${ }^{2} \mathrm{~F}_{4}(8)$ cannot occur as a composition factor of any 3-Brauer, 5-Brauer, 7-Brauer, 13-Brauer, 19-Brauer, 37-Brauer, or $\ell$-Brauer rational group, where $\ell \nmid\left|{ }^{2} \mathrm{~F}_{4}(8)\right|$, observe that ${ }^{2} \mathrm{~F}_{4}(8)$ has a liftable character $\chi$ of degree 35365873725 which satisfies $9 \mid[\mathbb{Q}(\chi): \mathbb{Q}]$, a violation of Theorem 5.1.1, as $\left|\operatorname{Out}\left({ }^{2} \mathrm{~F}_{4}(8)\right)\right|=3$. Likewise, ${ }^{2} \mathrm{~F}_{4}(8)$ cannot occur as a composition factor of any 109-Brauer rational group, as ${ }^{2} \mathrm{~F}_{4}(8)$ has a liftable character $\chi$ of degree 67629477825 which satisfies $6 \mid[\mathbb{Q}(\chi): \mathbb{Q}]$, another violation of Theorem 5.1.1.

Now, we have that ${ }^{2} \mathrm{G}_{2}(3)^{\prime} \cong \mathrm{L}_{2}(8)$, and thus, we must only prove the result for the prime 2. We follow the argument presented for $\mathrm{L}_{2}(7)$. In the case that $N_{G}\left(S_{1}\right) / C_{G}\left(S_{1}\right) \cong S$, choose an irrational character of degree 2 . In the case that $N_{G}\left(S_{1}\right) / C_{G}\left(S_{1}\right) \cong S .3$, choose the rational character of $S$ of degree 8 that extends to an irrational liftable character of S.3.

We have that the (non-defining characteristic) prime divisors of $\left|{ }^{2} \mathrm{G}_{2}(27)\right|$ are $2,7,13,19$, and 37 . To show that ${ }^{2} \mathrm{G}_{2}(27)$ cannot occur as a composition factor of any 2-Brauer, 7-Brauer, 13-Brauer, 19-Brauer, or $\ell$-Brauer rational group, where $\ell \nmid\left|{ }^{2} \mathrm{G}_{2}(27)\right|$, observe that ${ }^{2} \mathrm{G}_{2}(27)$ has a liftable character $\chi$ of degree 13832 which satisfies $6 \mid[\mathbb{Q}(\chi): \mathbb{Q}]$, a violation of Theorem 5.1.1, as $\left|\operatorname{Out}\left({ }^{2} \mathrm{G}_{2}(27)\right)\right|=3$. Likewise, ${ }^{2} \mathrm{G}_{2}(27)$ cannot occur as a composition factor of any 37-Brauer rational group, as ${ }^{2} \mathrm{G}_{2}(27)$ has a liftable character $\chi$ of degree 19684 which satisfies $6 \mid[\mathbb{Q}(\chi): \mathbb{Q}]$, another violation of Theorem 5.1.1. 


\begin{tabular}{|c|c|c|}
\hline Group & $\mathrm{G}_{2}(2)^{\prime}$ & ${ }^{3} \mathrm{D}_{4}(2)$ \\
\hline Prime $(\mathrm{s}) \ell$ & 3 & 3 \\
\hline QR Group & $\mathrm{G}_{2}(2)$ & ${ }^{3} \mathrm{D}_{4}(2) .3$ \\
\hline
\end{tabular}

\subsection{The Main Results}

We are now able to prove our main results.

Theorem 5.7.1. Let $G$ be a finite $\ell$-Brauer $m$-rational group. Then any non-abelian composition factor $S$ of $G$ is either an alternating group $\mathrm{A}_{n}, n \geq 5$, a sporadic group, a group of Lie type in characteristic $p=\ell$, or $S$ belongs to a finite set of groups of Lie type in characteristic $p \neq \ell, \mathcal{F}(m)$.

Proof. This follows immediately from Remark 5.1.6 and Theorems 5.2.1, 5.3.1, 5.4.1, $5.5 .1,5.5 .3,5.6 .1$.

Theorem 5.7.2. Let $G$ be a finite $\ell$-Brauer rational group. Then a finite non-abelian simple group $S$ can occur as a composition factor of $G$ only if $S$ is

- An alternating group $\mathrm{A}_{n}, n \geq 5$,

- A sporadic group from the following set:

$$
\left\{\mathrm{M}_{12}, \mathrm{M}_{22}, \mathrm{~J}_{2}, \mathrm{Fi}_{22}, \mathrm{HS}, \mathrm{Suz}, \mathrm{HN}\right\}
$$

- A group of Lie type in characteristic $\ell$,

- One of the groups of Lie type from the following set:

$$
\begin{gathered}
\left\{\mathrm{L}_{2}(7), \mathrm{L}_{2}(8), \mathrm{L}_{2}(25), \mathrm{L}_{3}(2), \mathrm{L}_{3}(4), \mathrm{L}_{4}(3),\right. \\
\mathrm{U}_{3}(3), \mathrm{U}_{3}(5), \mathrm{U}_{4}(2), \mathrm{U}_{4}(3), \mathrm{S}_{4}(4), \mathrm{S}_{6}(2) \\
\left.\mathrm{O}_{8}^{+}(2), \mathrm{O}_{8}^{+}(3), \mathrm{O}_{8}^{-}(2), \mathrm{G}_{2}(2)^{\prime},{ }^{3} \mathrm{D}_{4}(2)\right\}
\end{gathered}
$$


Proof. From Remark 5.1.6 and Corollaries 5.2.5, 5.3.5, 5.4.5, 5.5.2, 5.5.4, 5.6.2, it suffices to determine which sporadic groups occur as a composition factor of some $\ell$-Brauer rational group. We have that $\operatorname{Out}(S)$ is trivial for

$$
S \in\left\{\mathrm{M}_{11}, \mathrm{M}_{23}, \mathrm{M}_{24}, \mathrm{~J}_{1}, \mathrm{~J}_{4}, \mathrm{Co}_{1}, \mathrm{Co}_{2}, \mathrm{Co}_{3}, \mathrm{Fi}_{23}, \mathrm{Ru}, \mathrm{Ly}, \mathrm{Th}, \mathrm{B}, \mathrm{M}\right\}
$$

and thus for these groups, $S$ itself must be $\ell$-Brauer rational by Corollary 5.1.3. In many cases, the $\ell$-modular character tables are not recorded in the ATLAS or GAP, and thus, we may use Lemma 5.1.4, which we recall now:

Lemma 5.7.3. $S$ is $\ell$-Brauer rational if and only if $\chi(g) \in \mathbb{Z}$ for all $g \in S^{\circ}$ and $\chi \in \operatorname{Irr}(S)$.

We have that the prime divisors of $\left|\mathrm{M}_{11}\right|$ are $2,3,5$, and 11 . Choose an irrational character of degree 16 for all primes except 11, and an irrational character of degree 10 when $\ell=11$.

We have that the prime divisors of $\left|\mathrm{M}_{23}\right|$ are 2, 3, 5, 7, 11, and 23. When $\ell=2,5,7$, or $\ell \nmid\left|\mathrm{M}_{23}\right|$, choose an irrational character of degree 896, and when $\ell=3,11$, or 23 , choose an irrational character of degree 45 .

We have that the prime divisors of $\left|\mathrm{M}_{24}\right|$ are 2, 3, 5, 7, 11, and 23. When $\ell=3,5,7,11$, or $\ell \nmid\left|\mathrm{M}_{23}\right|$, choose an irrational character of degree 770 , when $\ell=23$, choose an irrational character of degree 990, and when $\ell=2$, choose the irrational character of degree 11.

We have that the prime divisors of $\left|\mathrm{J}_{1}\right|$ are $2,3,5,7,11$, and 19. When $\ell=2,3,5$, or $\ell \nmid\left|J_{1}\right|$, choose an irrational character of degree 120 , and when $\ell=7,11$, or 19 , choose an irrational character of degree 77.

We have that the prime divisors of $\left|\mathrm{J}_{4}\right|$ are $2,3,5,7,11,23,29,31,37$, and 43 . When $\ell=5,7,37$, or $\ell \nmid\left|J_{4}\right|$, choose an irrational character of degree 1445942610 . Now, for the remaining primes $\ell$, we see that a character of degree 1333 takes on irrationalities on the classes of order 7, and therefore, by Lemma 5.1.4, there exists 
an irrational 2-Brauer, 3-Brauer, 11-Brauer, 23-Brauer, 29-Brauer, 31-Brauer and 43Brauer character. Hence, $\mathrm{J}_{4}$ cannot occur as a composition factor of any 2-Brauer, 3-Brauer, 11-Brauer, 23-Brauer, 29-Brauer, 31-Brauer, or 43-Brauer rational group.

We have that the prime divisors of $\left|\mathrm{Co}_{1}\right|$ are $2,3,5,7,11,13$, and 23. When $\ell=7,11,13$, or $\ell \nmid\left|\mathrm{Co}_{1}\right|$, choose an irrational character of degree 673750 , when $\ell=23$, choose an irrational character of degree 2464749. As in the $\mathrm{J}_{4}$ case, we see that a character of degree 673750 takes on irrationalities on the classes of order 23 , and therefore there exists an irrational 2-Brauer, 3-Brauer, and 5-Brauer character.

We have that the prime divisors of $\left|\mathrm{Co}_{2}\right|$ are 2, 3, 5, 7, 11, and 23. When $\ell=5,7,11$, or $\ell \nmid\left|\mathrm{Co}_{2}\right|$, choose an irrational character of degree 9625, when $\ell=23$ or 3 , choose an irrational character of degree 91125 , and when $\ell=2$, choose the irrational character of degree 748 .

We have that the prime divisors of $\left|\mathrm{Co}_{3}\right|$ are 2, 3, 5, 7, 11, and 23. When $\ell=2,5,7,23$, or $\ell \nmid\left|\mathrm{Co}_{3}\right|$, choose an irrational character of degree 896 , when $\ell=11$, choose an irrational character of degree 9625, and when $\ell=3$, choose the irrational character of degree 126.

We have that the prime divisors of $\left|\mathrm{Fi}_{23}\right|$ are 2, 3, 5, 7, 11, 13, 17, and 23. When $\ell=2,5,7,11$, or $\ell \nmid\left|\mathrm{Fi}_{23}\right|$, choose an irrational character of degree 504627200, when $\ell=13,17$, or 23 , choose an irrational character of degree 9108736 . As in the $\mathrm{J}_{4}$ case, we see that a character of degree 153014400 takes on irrationalities on the classes of order 13, and therefore there exists an irrational 3-Brauer character.

We have that the prime divisors of $|\mathrm{Ru}|$ are 2, 3, 5, 7, 13, and 29. When $\ell=3,5,7,13,29$, or $\ell \nmid|\mathrm{Ru}|$, choose an irrational character of degree 378 , and when $\ell=2$, choose an irrational character of degree 8192 .

We have that the prime divisors of $|\mathrm{Ly}|$ are $2,3,5,7,11,31,37$, and 67 . When $\ell=7,31,37,67$, or $\ell \nmid|\mathrm{Ly}|$, choose an irrational character of degree 2480, and when $\ell=11$, choose an irrational character of degree 43110144. As in the $\mathrm{J}_{4}$ case, we see that a character of degree 43110144 takes on irrationalities on the classes of order 31 , 
and therefore there exists an irrational 2-Brauer, 3-Brauer, and 5-Brauer character.

We have that the prime divisors of $|\mathrm{Th}|$ are $2,3,5,7,13,19$, and 31 . When $\ell=13,19,31$, or $\ell \nmid|\mathrm{Th}|$, choose an irrational character of degree 27000 . As in the $\mathrm{J}_{4}$ case, we see that a character of degree 767637 takes on irrationalities on the classes of order 31, and therefore there exists an irrational 2-Brauer, 3-Brauer, 5-Brauer, and 7-Brauer character.

We have that the prime divisors of $|\mathrm{B}|$ are $2,3,5,7,11,13,17,19,23,31$, and 47. When $\ell=11,13,17,23,31,47$ or $\ell \nmid|B|$, choose an irrational character of degree 4097337118875. As in the $\mathrm{J}_{4}$ case, we see that a character of degree 252984703125 takes on irrationalities on the classes of order 23 , and therefore there exists an irrational 2-Brauer, 3-Brauer, 5-Brauer, 7-Brauer, 19-Brauer, and 47-Brauer character.

We have that the prime divisors of $|\mathrm{M}|$ are $2,3,5,7,11,13,17,19,23,29,31$, 41, 47, 59, and 71. When $\ell=17,19,23,31$, or $\ell \nmid|\mathrm{M}|$, choose an irrational character of degree 5514132424881463208443904. As in the $\mathrm{J}_{4}$ case, we see that a character of degree 8980616927734375 takes on irrationalities on the classes of order 23, and therefore there exists an irrational 2-Brauer, 3-Brauer, 5-Brauer, 7-Brauer, 11-Brauer, 13-Brauer, 29-Brauer, 41-Brauer, 47-Brauer, 59-Brauer, and 71-Brauer character.

Now, $\operatorname{Out}(S)$ has order 2 for

$$
S \in\left\{\mathrm{M}_{12}, \mathrm{M}_{22}, \mathrm{~J}_{2}, \mathrm{~J}_{3}, \mathrm{Fi}_{22}, \mathrm{Fi}_{24}^{\prime}, \mathrm{HS}, \mathrm{McL}, \mathrm{He}, \mathrm{Suz}, \mathrm{O}^{\prime} \mathrm{N}, \mathrm{HN}\right\} .
$$

We have that the prime divisors of $\left|\mathrm{M}_{12}\right|$ are 2, 3, 5, and 11. Using GAP, we see that $\mathrm{M}_{12}$ is 11-Brauer rational and $\mathrm{M}_{12} .2$ is 2-Brauer rational. To show that $\mathrm{M}_{12}$ cannot occur as a composition factor of a 5 -Brauer or $\ell$-Brauer rational group where $\ell \nmid$ $\left|\mathrm{M}_{12}\right|$, we follow the argument provided for $\mathrm{L}_{2}(7)$. In the case that $N_{G}\left(S_{1}\right) / C_{G}\left(S_{1}\right) \cong$ $S$, use an irrational character of degree 16 . In the case that $N_{G}\left(S_{1}\right) / C_{G}\left(S_{1}\right) \cong S .2$, use a rational character of $S$ of degree 120 that extends to a liftable irrational character of S.2. Likewise, to show that $\mathrm{M}_{12}$ cannot occur as a composition factor of a 3Brauer rational group, we follow the argument provided for $\mathrm{L}_{2}(7)$. In the case that 
$N_{G}\left(S_{1}\right) / C_{G}\left(S_{1}\right) \cong S$, use an irrational character of degree 15 . In the case that $N_{G}\left(S_{1}\right) / C_{G}\left(S_{1}\right) \cong S .2$, use a rational character of $S$ of degree 54 that extends to a liftable irrational character of S.2.

We have that the prime divisors of $\left|\mathrm{M}_{22}\right|$ are 2, 3, 5, 7, and 11. Using GAP, we see that $\mathrm{M}_{22} .2$ is 7 -Brauer rational. To see that $\mathrm{M}_{22}$ cannot occur as a composition factor of a 3-Brauer, 5-Brauer, 11-Brauer, or $\ell$-Brauer rational group where $\ell \nmid\left|\mathrm{M}_{22}\right|$, observe that $\mathrm{M}_{22}$ has an irrational character of degree 45 that extends to $\mathrm{M}_{22} \cdot 2$, a violation of Lemma 5.1.2, as $\left|\operatorname{Out}\left(\mathrm{M}_{22}\right)\right|=2$. Likewise, $\mathrm{M}_{22}$ cannot occur as a composition factor of a 2-Brauer rational group, as $\mathrm{M}_{22}$ has an irrational character of degree 10 that extends to $\mathrm{M}_{22} \cdot 2$.

We have that the prime divisors of $\left|\mathrm{J}_{2}\right|$ are $2,3,5$, and 7 . Using GAP, we see that $\mathrm{J}_{2}$ is 5 -Brauer rational and $\mathrm{J}_{2} .2$ is 2 -Brauer and 3 -Brauer rational. To show that $\mathrm{J}_{2}$ cannot occur as a composition factor of a 7 -Brauer or $\ell$-Brauer rational group where $\ell \nmid\left|\mathrm{J}_{2}\right|$, we follow the argument provided for $\mathrm{L}_{2}(7)$. In the case that $N_{G}\left(S_{1}\right) / C_{G}\left(S_{1}\right) \cong$ $S$, use an irrational character of degree 14 . In the case that $N_{G}\left(S_{1}\right) / C_{G}\left(S_{1}\right) \cong S .2$, use a rational character of $S$ of degree 336 that extends to a liftable irrational character of $S .2$.

We have that the prime divisors of $\left|J_{3}\right|$ are $2,3,5,17$, and 19 . To see that $J_{3}$ cannot occur as a composition factor of a 2-Brauer, 5-Brauer, or $\ell$-Brauer rational group where $\ell \nmid\left|J_{3}\right|$, observe that $\mathrm{J}_{3}$ has an irrational character of degree 1920 that extends to $\mathrm{J}_{3} .2$, a violation of Lemma 5.1 .2 , as $\left|\operatorname{Out}\left(\mathrm{J}_{3}\right)\right|=2$. To see that $\mathrm{J}_{3}$ cannot occur as a composition factor of a 3-Brauer rational group, observe that $\mathrm{J}_{3}$ has an irrational character of degree 1215 that extends to $\mathrm{J}_{3} .2$, another violation of Lemma 5.1.2. To see that $J_{3}$ cannot occur as a composition factor of a 17-Brauer rational group, observe that $\mathrm{J}_{3}$ has an irrational character of degree 379 that extends to $\mathrm{J}_{3} .2$, another violation of Lemma 5.1.2. Finally, $\mathrm{J}_{3}$ cannot occur as a composition factor of a 19-Brauer rational group, as $\mathrm{J}_{3}$ has an irrational character of degree 706 that extends to $\mathrm{J}_{3} .2$, another violation of Lemma 5.1 .2 . 
We have that the prime divisors of $\left|\mathrm{Fi}_{22}\right|$ are 2, 3, 5, 7, 11, and 13. Using GAP, we see that $\mathrm{Fi}_{22} .2$ is 2 -Brauer and 3 -Brauer rational. To show that $\mathrm{Fi}_{22}$ cannot occur as a composition factor of a 5-Brauer, 7-Brauer, or $\ell$-Brauer rational group where $\ell \nmid\left|\mathrm{J}_{2}\right|$, we follow the argument provided for $\mathrm{L}_{2}(7)$. In the case that $N_{G}\left(S_{1}\right) / C_{G}\left(S_{1}\right) \cong S$, use an irrational character of degree 582400. In the case that $N_{G}\left(S_{1}\right) / C_{G}\left(S_{1}\right) \cong S .2$, use a rational character of $S$ of degree 1360800 that extends to a liftable irrational character of S.2. By Lemma 5.2.7, $\mathrm{Fi}_{22}$ cannot occur as a composition factor of any 11-Brauer or 13-Brauer rational group. In the notation of Lemma 5.2.7, we may choose $\alpha^{\prime}$ to be an irrational character of degree 600600 , and $\alpha$ to be a character of degree 582400 or 138600 .

We have that the prime divisors of $\left|\mathrm{Fi}_{24}^{\prime}\right|$ are $2,3,5,7,11,13,17,23$, and 29. To see that $\mathrm{Fi}_{24}^{\prime}$ cannot occur as a composition factor of an 11-Brauer, 13-Brauer, 17-Brauer, 29-Brauer, or $\ell$-Brauer rational group where $\ell \nmid\left|\mathrm{Fi}_{24}^{\prime}\right|$, observe that $\mathrm{Fi}_{24}^{\prime}$ has an irrational character of degree 1603525 (found using GAP) that extends to $\mathrm{Fi}_{24}$, a violation of Lemma 5.1.2, as $\left|\operatorname{Out}\left(\mathrm{Fi}_{24}^{\prime}\right)\right|=2$. Likewise, when $\ell=23, \mathrm{Fi}_{24}^{\prime}$ has an irrational character of degree 89730653717 (again, found using GAP) that extends to $\mathrm{Fi}_{24}$, another violation of Lemma 5.1.2. To see that $\mathrm{Fi}_{24}^{\prime}$ cannot occur as a composition of any 2-Brauer, 3-Brauer, 5-Brauer, or 7-Brauer character, we must use some modular representation theory.

Definition 5.7.4. Let $\ell$ be a prime. An ordinary irreducible character $\chi \in \operatorname{Irr}(G)$ is said to have $\ell$-defect zero if $\ell \nmid(|G| / \chi(1))$. From Theorem 15.29 of [25], if $\chi$ is an $\ell$-defect zero character of $G$, then $\chi^{\circ} \in \operatorname{IBr}_{\ell}(G)$.

Using GAP, we see that $S=\mathrm{Fi}_{24}^{\prime}$ has an irrational 2-defect zero character of degree 197813862400 that extends to S.2, a violation of Lemma 5.1.2, and therefore $S$ cannot occur as a composition factor of a 2-Brauer rational group. Likewise, $S$ has an irrational 5, 7-defect zero character of degree 1603525 that extends to S.2, and $S$ cannot occur as a composition factor of any 5-Brauer or 7-Brauer rational group. 
Finally, we see that $S$ has a rational 3-defect zero character of degree 178514751987 that extends to an irrational character of $S .2$, a violation of Lemma 5.2.6. Therefore, $\mathrm{Fi}_{24}^{\prime}$ cannot occur as a composition factor of any 3-Brauer rational group.

We have that the prime divisors of $|\mathrm{HS}|$ are 2, 3, 5, 7, and 11. Using GAP, we see that HS.2 is 2-Brauer and 5-Brauer rational. To show that HS cannot occur as a composition factor of a 3-Brauer, 7-Brauer, or $\ell$-Brauer rational group where $\ell \nmid|\mathrm{HS}|$, we follow the argument provided for $\mathrm{L}_{2}(7)$. In the case that $N_{G}\left(S_{1}\right) / C_{G}\left(S_{1}\right) \cong S$, use an irrational character of degree 770 . In the case that $N_{G}\left(S_{1}\right) / C_{G}\left(S_{1}\right) \cong S .2$, use a rational character of $S$ of degree 2520 that extends to a liftable irrational character of S.2. By Lemma 5.2.7. HS cannot occur as a composition factor of any 11-Brauer rational group. In the notation of Lemma 5.2.7, we may choose $\alpha^{\prime}$ to be an irrational character of degree 770 , and $\alpha$ to be a character of degree 854 .

We have that the prime divisors of $|\mathrm{McL}|$ are 2, 3, 5, 7, and 11. To see that McL cannot occur as a composition factor of a 2-Brauer, 5-Brauer, 7-Brauer, or $\ell$ Brauer rational group where $\ell \nmid|\mathrm{McL}|$, observe that McL has an irrational character of degree 896 that extends to McL.2, a violation of Lemma 5.1.2, as $\mid$ Out(McL) $\mid=2$. Likewise, McL cannot occur as a composition factor of a 3-Brauer rational group as it contains an irrational character of degree 104 that extends to McL, another violation of Lemma 5.1.2. Finally, to show that McL cannot occur as a composition factor of an 11-Brauer rational group, we follow the argument provided for $\mathrm{L}_{2}(7)$. In the case that $N_{G}\left(S_{1}\right) / C_{G}\left(S_{1}\right) \cong S$, use an irrational character of degree 770 . In the case that $N_{G}\left(S_{1}\right) / C_{G}\left(S_{1}\right) \cong S .2$, use a rational character of $S$ of degree 3520 that extends to a liftable irrational character of S.2.

We have that the prime divisors of $|\mathrm{He}|$ are 2, 3, 5, 7, and 17. To see that He cannot occur as a composition factor of a 2 -Brauer or $\ell$-Brauer rational group where $\ell \nmid|\mathrm{He}|$, observe that He has an irrational character of degree 21504 that extends to He.2, a violation of Lemma 5.1.2, as $|\operatorname{Out}(\mathrm{He})|=2$. To show that He cannot occur as a composition factor of a 3-Brauer, 5-Brauer, or 17-Brauer rational group, 
we follow the argument provided for $\mathrm{L}_{2}(7)$. In the case that $N_{G}\left(S_{1}\right) / C_{G}\left(S_{1}\right) \cong S$, use an irrational character of degree 51. In the case that $N_{G}\left(S_{1}\right) / C_{G}\left(S_{1}\right) \cong S .2$, use a rational character of $S$ of degree 7650 that extends to a liftable irrational character of S.2. By Lemma 5.2.7, He cannot occur as a composition factor of any 7-Brauer rational group. In the notation of Lemma 5.2.7, we may choose $\alpha^{\prime}$ to be an irrational character of degree 1029, and $\alpha$ to be a character of degree 50.

We have that the prime divisors of $\mid$ Suz $\mid$ are 2, 3, 5, 7, 11, and 13. Using GAP, we see that Suz.2 is 2-Brauer and 5-Brauer rational. To show that Suz cannot occur as a composition factor of a 7-Brauer, 11-Brauer, or $\ell$-Brauer rational group where $\ell \nmid$ $\mid$ Suz|, we follow the argument provided for $\mathrm{L}_{2}(7)$. In the case that $N_{G}\left(S_{1}\right) / C_{G}\left(S_{1}\right) \cong$ $S$, use an irrational character of degree 5005. In the case that $N_{G}\left(S_{1}\right) / C_{G}\left(S_{1}\right) \cong S .2$, use a rational character of $S$ of degree 197120 that extends to a liftable irrational character of S.2. By Lemma 5.2.7, Suz cannot occur as a composition factor of any 3-Brauer or 13-Brauer rational group. In the notation of Lemma 5.2.7, we may choose $\alpha^{\prime}$ to be an irrational character of degree 5103 or 5005, and $\alpha$ to be a character of degree 64 or 57651.

We have that the prime divisors of $\left|\mathrm{O}^{\prime} \mathrm{N}\right|$ are $2,3,5,7,11,19$, and 31. To show that O'N cannot occur as a composition factor of a 2-Brauer, 3-Brauer, 5-Brauer, or $\ell$-Brauer rational group, where $\ell \nmid\left|\mathrm{O}^{\prime} \mathrm{N}\right|$, observe that $\mathrm{O}^{\prime} \mathrm{N}$ has a liftable character $\chi$ of degree 207360 that satisfies $3 \mid[\mathbb{Q}(\chi): \mathbb{Q}]$, a clear violation of Theorem 5.1.1. To show that O'N cannot occur as a composition factor of an 11-Brauer or 19-Brauer group, observe that O'N has an irrational character of degree 234080 that extends to a liftable character of O'N.2. By Lemma 5.2.7. O'N cannot occur as a composition factor of any 7-Brauer or 31-Brauer rational group. In the notation of Lemma 5.2.7, we may choose $\alpha^{\prime}$ to be an irrational character of degree 406 or 25916, and $\alpha$ to be a character of degree 15807 or 13376.

We have that the prime divisors of $|\mathrm{HN}|$ are 2, 3, 5, 7, 11, and 19. Using GAP, we see that HN.2 is 2-Brauer rational. To show that HN cannot occur as a composition 
factor of a 3-Brauer, 7-Brauer, or $\ell$-Brauer rational group, where $\ell \nmid|\mathrm{HN}|$, observe that HN has an irrational character of degree 5103000 that extends to HN.2, a violation of Lemma 5.1.2. To show that HN cannot occur as a composition factor of an 11Brauer rational group, we follow the argument provided for $\mathrm{L}_{2}(7)$. In the case that $N_{G}\left(S_{1}\right) / C_{G}\left(S_{1}\right) \cong S$, use an irrational character of degree 133 . In the case that $N_{G}\left(S_{1}\right) / C_{G}\left(S_{1}\right) \cong S .2$, use a rational character of $S$ of degree 2661120 that extends to a liftable irrational character of S.2. By Lemma 5.2.7, HN cannot occur as a composition factor of any 5-Brauer or 19-Brauer rational group. In the notation of Lemma 5.2.7, we may choose $\alpha^{\prime}$ to be an irrational character of degree 656250 or 133 , and $\alpha$ to be a character of degree 133 or 656250 .

We believe that it is also possible to show that only finitely many groups of Lie type in characteristic $\ell$ can occur as a composition factor of some $\ell$-Brauer $m$-rational group. In order to show this, we will prove a different reduction lemma.

Lemma 5.7.5. Suppose that $S$ is a non-abelian composition factor of an $\ell$-Brauer $m$-rational group. Let $\alpha \in \operatorname{IBr}_{\ell}(S)$ be afforded by $\Phi: S \rightarrow \mathrm{GL}_{L}(\mathbb{F}), \alpha(1)=L$. Let $g \in S^{\circ}$, and assume $\xi$ is an eigenvalue of $\Phi(g),|\xi|=N$. Then there exists $R \leq \operatorname{Out}(S)$ such that

$$
\phi(N) \leq m \cdot j \cdot L
$$

where $\left\{\alpha_{1}, \ldots, \alpha_{j}\right\}$ is the $R$-orbit of $\alpha$.

Proof. Let $G$ be an $\ell$-Brauer $m$-rational group with $N=S_{1} \times \cdots \times S_{n} \cong S^{n}$ a minimal normal subgroup. Define $R \leq \operatorname{Out}(S)$ as in Lemma 5.1.2, and define $\beta=$ $\alpha \otimes 1_{S_{2}} \otimes \cdots \otimes 1_{S_{n}}$. Let $\chi \in \operatorname{IBr}_{\ell}(G \mid \beta)$, and define $\lambda:\langle g\rangle \rightarrow \mathbb{C}$ by $\lambda(g)=\xi$. Then, we see that

$$
\chi_{\langle g\rangle}=\left.\chi_{N_{G}\left(S_{1}\right)}\right|_{\langle g\rangle}=\left(e \sum_{i=1}^{t} \beta^{i}\right)_{\langle g\rangle}=\left(e \sum_{i=1}^{j} \beta^{i}+\sum_{i=j+1}^{t} \beta^{i}\right)_{\langle g\rangle},
$$


where $\beta^{1}, \ldots, \beta^{t}$ is the full $G$-orbit of $\beta$, and $\beta^{1}, \ldots, \beta^{j}$ corresponds to the $R$-orbit of $\alpha$. Now, for $\beta^{i}$ with $j+1 \leq i \leq t, \beta^{i}(g)=\alpha(1)$, and hence, the only possible irrational contribution to $\chi_{\langle g\rangle}$ comes from the character $\mu$ of $\langle g\rangle$ defined by

$$
\mu:=\sum_{i=1}^{j} \beta_{\langle g\rangle}^{i}=\sum_{1 \leq i \leq j, 1 \leq k \leq L} \lambda_{i k} .
$$

Without loss, say $\lambda_{11}=\lambda$. Let $\epsilon$ be a primitive $|g|$-th root of unity, and define $\Gamma=\operatorname{Gal}(\mathbb{Q}(\epsilon) / \mathbb{Q})$. Let $\Gamma_{1} \leq \Gamma$ have fixed field $\mathbb{Q}(\mu)$ and $\Gamma_{2} \leq \Gamma$ have fixed field $\mathbb{Q}(\xi)$. Then, for any $\sigma \in \Gamma_{1}$, we have $\mu=\mu^{\sigma}$, and therefore, $\lambda^{\sigma}$ is also a constituent of $\mu$. Thus, the $\Gamma_{1}$-orbit of $\lambda$ has length at most $j \cdot L$. Further, $\lambda(g)^{\sigma}=\lambda(g)$ iff $\xi^{\sigma}=\xi$ iff $\sigma=1$. That is, the stabilizer of $\lambda$ in $\Gamma_{1}$ is $\Gamma_{1} \cap \Gamma_{2}$. Thus, by the orbit-stabilizer theorem, we have that

$$
j \cdot L \geq\left|\operatorname{Orb}_{\Gamma_{1}}(\lambda)\right|=\left[\Gamma_{1}: \Gamma_{1} \cap \Gamma_{2}\right]=\left[\Gamma_{1} \Gamma_{2}: \Gamma_{2}\right] \geq \frac{\phi(N)}{m},
$$

as $\left[\mathbb{Q}\left(\chi_{\langle g\rangle}\right): \mathbb{Q}\right]=[\mathbb{Q}(\mu): \mathbb{Q}] \mid m$, and $[\mathbb{Q}(\xi): \mathbb{Q}]=\phi(N)$. Therefore $\phi(N) \leq$ $m \cdot j \cdot L$.

This lemma is tailored specifically for the groups of Lie type in characteristic $\ell$, since not many irreducible $\ell$-Brauer characters are liftable. Furthermore, as we can see in GAP, there are typically irreducible $\ell$-Brauer characters of small degrees that take on many different irrationalities. We will use these characters and semisimple elements $g \in S^{\circ}$ as before to determine that only finitely many groups of Lie type in characteristic $\ell$ can occur as a composition factor of some $\ell$-Brauer $m$-rational group, giving us the following conjecture.

Conjecture 1. Let $G$ be a finite $\ell$-Brauer m-rational group. Then any non-abelian composition factor $S$ of $G$ is either an alternating group $\mathrm{A}_{n}, n \geq 5$, a sporadic group, or $S$ belongs to a finite set of groups of Lie type, $\mathcal{F}(m)$. 


\section{A. Appendix}

\section{A.1. CHEVIE Character Table}

In this section, we provide a few of the known character tables available in CHEVIE that we found useful in our work, [19].

$\operatorname{PSL}_{2}(q), q \equiv 1 \bmod 4: \zeta_{1}$ is a primitive $q-1$-th root of unity and $\xi_{1}$ is a primitive $q+1$-th root of unity.

\begin{tabular}{|c|c|c|c|c|}
\hline $\mathrm{PSL}_{2}(q)$ & $C_{1}$ & $C_{2}$ & $C_{3}$ & $C_{4}(i)$ \\
\hline$\chi_{1}$ & 1 & 1 & 1 & 1 \\
\hline$\chi_{2}$ & $q$ & 0 & 0 & 1 \\
\hline$\chi_{3}$ & $\frac{1}{2}(q+1)$ & $\frac{1}{2}-\frac{1}{2} \sqrt{q}$ & $\frac{1}{2}+\frac{1}{2} \sqrt{q}$ & $(-1)^{i}$ \\
\hline$\chi_{4}$ & $\frac{1}{2}(q+1)$ & $\frac{1}{2}+\frac{1}{2} \sqrt{q}$ & $\frac{1}{2}-\frac{1}{2} \sqrt{q}$ & $(-1)^{i}$ \\
\hline$\chi_{5}(k)$ & $q+1$ & 1 & 1 & $\zeta_{1}^{2 i k}+\zeta_{1}^{-2 i k}$ \\
\hline$\chi_{6}(k)$ & $q-1$ & -1 & -1 & 0 \\
\hline
\end{tabular}

\begin{tabular}{|c|c|c|}
\hline $\mathrm{PSL}_{2}(q)$ & $C_{5}(i)$ & $C_{6}$ \\
\hline$\chi_{1}$ & 1 & 1 \\
\hline$\chi_{2}$ & -1 & 1 \\
\hline$\chi_{3}$ & 0 & $(-1)^{\frac{1}{4}(q-1)}$ \\
\hline$\chi_{4}$ & 0 & $(-1)^{\frac{1}{4}(q-1)}$ \\
\hline$\chi_{5}(k)$ & 0 & $(-1)^{k}+(-1)^{-k}$ \\
\hline$\chi_{6}(k)$ & $-\xi_{1}^{2 i k}-\xi_{1}^{-2 i k}$ & 0 \\
\hline
\end{tabular}

$\operatorname{PSL}_{2}(q), q \equiv 3 \bmod 4: \zeta_{1}$ is a primitive $q-1$-th root of unity, $\epsilon_{4}$ is a primitive 4 -th root of unity, and $\xi_{1}$ is a primitive $q+1$-th root of unity.

\begin{tabular}{|c|c|c|c|c|}
\hline $\mathrm{PSL}_{2}(q)$ & $C_{1}$ & $C_{2}$ & $C_{3}$ & $C_{4}(i)$ \\
\hline$\chi_{1}$ & 1 & 1 & 1 & 1 \\
\hline$\chi_{2}$ & $q$ & 0 & 0 & 1 \\
\hline$\chi_{3}$ & $\frac{1}{2}(q-1)$ & $-\frac{1}{2}+\frac{1}{2} \sqrt{q} \epsilon_{4}$ & $-\frac{1}{2}-\frac{1}{2} \sqrt{q} \epsilon_{4}$ & 0 \\
\hline$\chi_{4}$ & $\frac{1}{2}(q-1)$ & $-\frac{1}{2}-\frac{1}{2} \sqrt{q} \epsilon_{4}$ & $-\frac{1}{2}+\frac{1}{2} \sqrt{q} \epsilon_{4}$ & 0 \\
\hline$\chi_{5}(k)$ & $q+1$ & 1 & 1 & $\zeta_{1}^{2 i k}+\zeta_{1}^{-2 i k}$ \\
\hline$\chi_{6}(k)$ & $q-1$ & -1 & -1 & 0 \\
\hline
\end{tabular}




\begin{tabular}{|c|c|c|}
\hline $\mathrm{PSL}_{2}(q)$ & $C_{5}(i)$ & $C_{6}$ \\
\hline$\chi_{1}$ & 1 & 1 \\
\hline$\chi_{2}$ & -1 & -1 \\
\hline$\chi_{3}$ & $-(-1)^{i}$ & $-(-1)^{\frac{1}{4}(q+1)}$ \\
\hline$\chi_{4}$ & $-(-1)^{i}$ & $-(-1)^{\frac{1}{4}(q+1)}$ \\
\hline$\chi_{5}(k)$ & 0 & 0 \\
\hline$\chi_{6}(k)$ & $-\xi_{1}^{2 i k}-\xi_{1}^{-2 i k}$ & $-(-1)^{k}-(-1)^{-k}$ \\
\hline
\end{tabular}

$\operatorname{PSL}_{2}(q), q$ even: $\zeta_{1}$ is a primitive $q-1$-th root of unity and $\xi_{1}$ is a primitive $q+1$-th root of unity.

\begin{tabular}{|c|c|c|c|c|}
\hline $\mathrm{PSL}_{2}(q)$ & $C_{1}$ & $C_{2}$ & $C_{3}(a)$ & $C_{4}(a)$ \\
\hline$\chi_{1}$ & 1 & 1 & 1 & 1 \\
\hline$\chi_{2}$ & $q$ & 0 & 1 & -1 \\
\hline$\chi_{3}(n)$ & $q+1$ & 1 & $\zeta_{1}^{a n}+\zeta_{1}^{-a n}$ & 0 \\
\hline$\chi_{4}(n)$ & $q-1$ & -1 & 0 & $-\xi_{1}^{a n}-\xi_{1}^{-a n}$ \\
\hline
\end{tabular}

$\operatorname{PSL}_{3}(q), q \equiv 0,2 \bmod 3: \zeta_{1}$ is a primitive $q-1$-th root of unity, $\zeta_{2}$ is a primitive $q^{2}-1$-th root of unity and $\phi_{3}$ is a primitive $q^{2}+q+1$-th root of unity.

\begin{tabular}{|c|c|c|c|c|}
\hline $\mathrm{PSL}_{3}(q)$ & $C_{1}$ & $C_{2}$ & $C_{3}$ & $C_{4}(a)$ \\
\hline$\chi_{1}$ & 1 & 1 & 1 & 1 \\
\hline$\chi_{2}$ & $q^{2}+q$ & $q$ & 0 & $q+1$ \\
\hline$\chi_{3}$ & $q^{3}$ & 0 & 0 & $q$ \\
\hline$\chi_{4}(n)$ & $q^{2}+q+1$ & $q+1$ & 1 & $q \zeta_{1}^{a n}+\zeta_{1}^{a n}+\zeta_{1}^{-2 a n}$ \\
\hline$\chi_{5}(n)$ & $q\left(q^{2}+q+1\right)$ & $q$ & 0 & $q \zeta_{1}^{a n}+\zeta_{1}^{a n}+q \zeta_{1}^{-2 a n}$ \\
\hline$\chi_{6}(n, m)$ & $(q+1)\left(q^{2}+q+1\right)$ & $2 q+1$ & 1 & $(q+1)\left(\zeta_{1}^{a n-2 a m}+\zeta_{1}^{a m-2 a n}+\zeta_{1}^{a n+a m}\right)$ \\
\hline$\chi_{7}(n)$ & $(q-1)\left(q^{2}+q+1\right)$ & -1 & -1 & $(q-1) \zeta_{1}^{a n}$ \\
\hline$\chi_{8}(n)$ & $(q-1)^{2}(q+1)$ & $-(q-1)$ & 1 & 0 \\
\hline
\end{tabular}




\begin{tabular}{|c|c|c|}
\hline $\mathrm{PSL}_{3}(q)$ & $C_{5}(a)$ & $C_{6}(a, b)$ \\
\hline$\chi_{1}$ & 1 & 1 \\
\hline$\chi_{2}$ & 1 & 2 \\
\hline$\chi_{3}$ & 0 & 1 \\
\hline$\chi_{4}(n)$ & $\zeta_{1}^{a n}+\zeta_{1}^{-2 a n}$ & $\zeta_{1}^{a n}+\zeta_{1}^{b n}+\zeta_{1}^{-n(a+b)}$ \\
\hline$\chi_{5}(n)$ & $\zeta_{1}^{a n}$ & $\zeta_{1}^{a n}+\zeta_{1}^{b n}+\zeta_{1}^{-n(a+b)}$ \\
\hline$\chi_{6}(n, m)$ & $\zeta_{1}^{a n-2 a m}+\zeta_{1}^{a m-2 a n}+\zeta_{1}^{a n+a m}$ & $\begin{array}{l}\zeta_{1}^{-a m-b m+a n}+\zeta_{1}^{-a m-b m+b n}+\zeta_{1}^{-a m-a n-b n} \\
+\zeta_{1}^{b m-a n-b n}+\zeta_{1}^{a m+b n}+\zeta_{1}^{b m+a n}\end{array}$ \\
\hline$\chi_{7}(n)$ & $-\zeta_{1}^{a n}$ & 0 \\
\hline$\chi_{8}(n)$ & 0 & 0 \\
\hline
\end{tabular}

\begin{tabular}{|c|c|c|}
\hline $\mathrm{PSL}_{3}(q)$ & $C_{7}(a)$ & $C_{8}(a)$ \\
\hline$\chi_{1}$ & 1 & 1 \\
\hline$\chi_{2}$ & 0 & -1 \\
\hline$\chi_{3}$ & -1 & 0 \\
\hline$\chi_{4}(n)$ & $\zeta_{1}^{a n}$ & 0 \\
\hline$\chi_{5}(n)$ & $-\zeta_{1}^{a n}$ & 0 \\
\hline$\chi_{6}(n, m)$ & 0 & 0 \\
\hline$\chi_{7}(n)$ & $-\zeta_{2}^{a n}-\zeta_{2}^{q a n}$ & $\phi_{3}^{a n}+\phi_{3}^{q a n}+\phi_{3}^{-a n-q a n}$ \\
\hline$\chi_{8}(n)$ & 0 & \\
\hline
\end{tabular}

$\operatorname{PSL}_{3}(q), q \equiv 1 \bmod 3: \epsilon_{3}$ is a primitive $3 \mathrm{rd}$ root of unity, $\zeta_{1}$ is a primitive $q-1$-th root of unity, $\zeta_{2}$ is a primitive $q^{2}-1$-th root of unity and $\phi_{3}$ is a primitive $q^{2}+q+1$-th root of unity.

\begin{tabular}{|c|c|c|c|c|c|}
\hline $\mathrm{PSL}_{3}(q)$ & $C_{1}$ & $C_{2}$ & $C_{3}$ & $C_{4}$ & $C_{5}$ \\
\hline$\chi_{1}$ & 1 & 1 & 1 & 1 & 1 \\
\hline$\chi_{2}$ & $q^{2}+q$ & $q$ & 0 & 0 & 0 \\
\hline$\chi_{3}$ & $q^{3}$ & 0 & 0 & 0 & 0 \\
\hline$\chi_{4}(n)$ & $q^{2}+q+1$ & $q+1$ & 1 & 1 & 1 \\
\hline$\chi_{5}(n)$ & $q\left(q^{2}+q+1\right)$ & $q$ & 0 & 0 & 0 \\
\hline$\chi_{6}$ & $\frac{1}{3}(q+1)\left(q^{2}+q+1\right)$ & $\frac{1}{3}(2 q+1)$ & $\frac{1}{3}(2 q+1)$ & $-\frac{1}{3}(q-1)$ & $-\frac{1}{3}(q-1)$ \\
\hline$\chi_{7}$ & $\frac{1}{3}(q+1)\left(q^{2}+q+1\right)$ & $\frac{1}{3}(2 q+1)$ & $-\frac{1}{3}(q-1)$ & $\frac{1}{3}(2 q+1)$ & $-\frac{1}{3}(q-1)$ \\
\hline$\chi_{8}$ & $\frac{1}{3}(q+1)\left(q^{2}+q+1\right)$ & $\frac{1}{3}(2 q+1)$ & $-\frac{1}{3}(q-1)$ & $-\frac{1}{3}(q-1)$ & $\frac{1}{3}(2 q+1)$ \\
\hline$\chi_{9}(n, m)$ & $(q+1)\left(q^{2}+q+1\right)$ & $2 q+1$ & 1 & 1 & 1 \\
\hline$\chi_{10}(n)$ & $(q-1)\left(q^{2}+q+1\right)$ & -1 & -1 & -1 & -1 \\
\hline$\chi_{11}(n)$ & $(q-1)^{2}(q+1)$ & $-(q-1)$ & 1 & 1 & 1 \\
\hline
\end{tabular}




\begin{tabular}{|c|c|c|}
\hline $\mathrm{PSL}_{3}(q)$ & $C_{6}(a)$ & $C_{7}(a)$ \\
\hline$\chi_{1}$ & 1 & 1 \\
\hline$\chi_{2}$ & $q+1$ & 1 \\
\hline$\chi_{3}$ & $q$ & $\zeta_{1}^{3 a n}+\zeta_{1}^{-6 a n}$ \\
\hline$\chi_{4}(n)$ & $q \zeta_{1}^{3 a n}+\zeta_{1}^{3 a n}+\zeta_{1}^{-6 a n}$ & $\zeta_{1}^{3 a n}$ \\
\hline$\chi_{5}(n)$ & $q \zeta_{1}^{3 a n}+\zeta_{1}^{3 a n}+\zeta_{1}^{-6 a n}$ & 1 \\
\hline$\chi_{6}$ & $q+1$ & 1 \\
\hline$\chi_{7}$ & $q+1$ & $-\zeta_{1}^{3 a n}$ \\
\hline$\chi_{8}$ & $q+1$ & 0 \\
\hline$\chi_{9}(n, m)$ & $(q+1)\left(\zeta_{1}^{-3 a(-n+m)}+\zeta_{1}^{3 a(-2 n+m)}+\zeta_{1}^{3 a n}\right)$ & $\left.\zeta_{1}^{-3 a(-n+m)}+\zeta_{1}^{3 a(-2 n+m)}+\zeta_{1}^{3 a n}\right)$ \\
\hline$\chi_{10}(n)$ & $(q-1) \zeta_{1}^{3 a n}$ & \\
\hline$\chi_{11}(n)$ & 0 & \\
\hline
\end{tabular}

\begin{tabular}{|c|c|c|}
\hline $\mathrm{PSL}_{3}(q)$ & $C_{8}$ & $C_{9}(a, b)$ \\
\hline$\chi_{1}$ & 1 & 1 \\
\hline$\chi_{2}$ & 2 & 2 \\
\hline$\chi_{3}$ & 1 & 1 \\
\hline$\chi_{4}(n)$ & 3 & $\zeta_{1}^{3 a n}+\zeta_{1}^{3 b n}+\zeta_{1}^{-3 n(a+b)}$ \\
\hline$\chi_{5}(n)$ & 3 & $\zeta_{1}^{3 a n}+\zeta_{1}^{3 b n}+\zeta_{1}^{-3 n(a+b)}$ \\
\hline$\chi_{6}$ & $\epsilon_{3}^{\frac{1}{3}(q-1)}+\epsilon_{3}^{\frac{2}{3}(q-1)}$ & $\epsilon_{3}^{a-b}+\epsilon_{3}^{b-a}$ \\
\hline$\chi_{7}$ & $\epsilon_{3}^{\frac{1}{3}(q-1)}+\epsilon_{3}^{\frac{2}{3}(q-1)}$ & $\epsilon_{3}^{2 a+b}+\epsilon_{3}^{-2 a-b}$ \\
\hline$\chi_{8}$ & $\epsilon_{3}^{\frac{1}{3}(q-1)}+\epsilon_{3}^{\frac{2}{3}(q-1)}$ & $\epsilon_{3}^{2 b+a}+\epsilon_{3}^{-a-2 b}$ \\
\hline$\chi_{9}(n, m)$ & $3 \epsilon_{3}^{m}+3 \epsilon_{3}^{2 m}$ & $\begin{array}{l}\zeta_{1}^{2 a m-3 a n-3 b n+b m}+\zeta_{1}^{2 b m-3 a n-3 b n+a m}+\zeta_{1}^{-2 a m-b m+3 a n} \\
+\zeta_{1}^{-a m-2 b m+3 b n}+\zeta_{1}^{b m+3 a n-a m}+\zeta_{1}^{a m+3 b n-b m}\end{array}$ \\
\hline$\chi_{10}(n)$ & 0 & 0 \\
\hline$\chi_{11}(n)$ & 0 & 0 \\
\hline
\end{tabular}

\begin{tabular}{|c|c|c|}
\hline $\operatorname{PSL}_{3}(q)$ & $C_{10}(a)$ & $C_{11}(a)$ \\
\hline$\chi_{1}$ & 1 & 1 \\
\hline$\chi_{2}$ & 0 & -1 \\
\hline$\chi_{3}$ & -1 & 1 \\
\hline$\chi_{4}(n)$ & $\zeta_{1}^{3 a n}$ & 0 \\
\hline$\chi_{5}(n)$ & $-\zeta_{1}^{3 a n}$ & 0 \\
\hline$\chi_{6}$ & 0 & 0 \\
\hline$\chi_{7}$ & 0 & 0 \\
\hline$\chi_{8}$ & 0 & 0 \\
\hline$\chi_{9}(n, m)$ & 0 & 0 \\
\hline$\chi_{10}(n)$ & $-\zeta_{2}^{3 a n}-\zeta_{2}^{3 q a n}$ & $\phi_{3}^{3 a n}+\phi_{3}^{3 q a n}+\phi_{3}^{-3 a n-3 q a n}$ \\
\hline$\chi_{11}(n)$ & 0 &
\end{tabular}


$\operatorname{PSU}_{3}(q), q \equiv 0,1 \bmod 3: \xi_{1}$ is a primitive $q+1$-th root of unity, $\zeta_{2}$ is a primitive $q^{2}-1$-th root of unity and $\phi_{6}$ is a primitive $q^{2}-q+1$-th root of unity.

\begin{tabular}{|c|c|c|c|c|}
\hline $\mathrm{PSU}_{3}(q)$ & $C_{1}$ & $C_{2}$ & $C_{3}$ & $C_{4}(a)$ \\
\hline$\chi_{1}$ & 1 & 1 & 1 & 1 \\
\hline$\chi_{2}$ & $q^{2}+q$ & $-q$ & 0 & $-(q-1)$ \\
\hline$\chi_{3}$ & $q^{3}$ & 0 & 0 & $q$ \\
\hline$\chi_{4}(n)$ & $q^{2}-q+1$ & $-(q-1)$ & 1 & $q \xi_{1}^{a n}+\xi_{1}^{a n}+\xi_{1}^{-2 a n}$ \\
\hline$\chi_{5}(n)$ & $q\left(q^{2}-q+1\right)$ & $q$ & 0 & $q \xi_{1}^{a n}-\xi_{1}^{a n}+q \xi_{1}^{-2 a n}$ \\
\hline$\chi_{6}(n, m)$ & $(q-1)\left(q^{2}-q+1\right)$ & $2 q-1$ & -1 & $(q-1)\left(\xi_{1}^{a n-2 a m}+\xi_{1}^{a m-2 a n}+\xi_{1}^{a n+a m}\right)$ \\
\hline$\chi_{7}(n)$ & $(q+1)\left(q^{2}-q+1\right)$ & 1 & 1 & $(q+1) \xi_{1}^{a n}$ \\
\hline$\chi_{8}(n)$ & $(q-1)(q+1)^{2}$ & $-(q+1)$ & -1 & 0 \\
\hline
\end{tabular}

\begin{tabular}{|c|c|c|}
\hline $\mathrm{PSU}_{3}(q)$ & $C_{5}(a)$ & $C_{6}(a, b)$ \\
\hline$\chi_{1}$ & 1 & 1 \\
\hline$\chi_{2}$ & 1 & 2 \\
\hline$\chi_{3}$ & 0 & -1 \\
\hline$\chi_{4}(n)$ & $\xi_{1}^{a n}+\xi_{1}^{-2 a n}$ & $\xi_{1}^{a n}+\xi_{1}^{b n}+\xi_{1}^{-n(a+b)}$ \\
\hline$\chi_{5}(n)$ & $-\xi_{1}^{a n}$ & $-\xi_{1}^{a n}-\xi_{1}^{b n}-\xi_{1}^{-n(a+b)}$ \\
\hline$\chi_{6}(n, m)$ & $-\xi_{1}^{a n-2 a m}-\xi_{1}^{a m-2 a n}-\xi_{1}^{a n+a m}$ & $\begin{array}{l}-\xi_{1}^{-a m-b m+a n}-\xi_{1}^{-a m-b m+b n}-\xi_{1}^{a m-a n-b n} \\
-\xi_{1}^{b m-a n-b n}-\xi_{1}^{a m+b n}-\xi_{1}^{b m+a n}\end{array}$ \\
\hline$\chi_{7}(n)$ & $\xi_{1}^{a n}$ & 0 \\
\hline$\chi_{8}(n)$ & 0 & 0 \\
\hline
\end{tabular}

\begin{tabular}{|c|c|c|}
\hline $\mathrm{PSU}_{3}(q)$ & $C_{7}(a)$ & $C_{8}(a)$ \\
\hline$\chi_{1}$ & 1 & 1 \\
\hline$\chi_{2}$ & 0 & -1 \\
\hline$\chi_{3}$ & 1 & -1 \\
\hline$\chi_{4}(n)$ & $\xi_{1}^{a n}$ & 0 \\
\hline$\chi_{5}(n)$ & $\xi_{1}^{a n}$ & 0 \\
\hline$\chi_{6}(n, m)$ & 0 & 0 \\
\hline$\chi_{7}(n)$ & $\zeta_{2}^{a n}+\zeta_{2}^{-q a n}$ & 0 \\
\hline$\chi_{8}(n)$ & 0 & $-\phi_{6}^{a n}-\phi_{6}^{-q a n}-\phi_{6}^{q^{2} a n}$ \\
\hline
\end{tabular}

$\operatorname{PSU}_{3}(q), q \equiv 2 \bmod 3: \epsilon_{3}$ is a primitive 3 rd root of unity, $\xi_{1}$ is a primitive $q+1$-th root of unity, $\zeta_{2}$ is a primitive $q^{2}-1$-th root of unity and $\phi_{6}$ is a primitive $q^{2}-q+1$-th root of unity. 


\begin{tabular}{|c|c|c|c|c|c|}
\hline $\mathrm{PSU}_{3}(q)$ & $C_{1}$ & $C_{2}$ & $C_{3}$ & $C_{4}$ & $C_{5}$ \\
\hline$\chi_{1}$ & 1 & 1 & 1 & 1 & 1 \\
\hline$\chi_{2}$ & $q^{2}+q$ & $-q$ & 0 & 0 & 0 \\
\hline$\chi_{3}$ & $q^{3}$ & 0 & 0 & 0 & 0 \\
\hline$\chi_{4}(n)$ & $q^{2}-q+1$ & $-(q-1)$ & 1 & 1 & 1 \\
\hline$\chi_{5}(n)$ & $q\left(q^{2}-q+1\right)$ & $q$ & 0 & 0 & 0 \\
\hline$\chi_{6}(n)$ & $(q+1)\left(q^{2}-q+1\right)$ & 1 & 1 & 1 & 1 \\
\hline$\chi_{7}$ & $\frac{1}{3}(q-1)\left(q^{2}-q+1\right)$ & $\frac{1}{3}(2 q-1)$ & $\frac{1}{3}(2 q-1)$ & $-\frac{1}{3}(q+1)$ & $-\frac{1}{3}(q+1)$ \\
\hline$\chi_{8}$ & $\frac{1}{3}(q-1)\left(q^{2}-q+1\right)$ & $\frac{1}{3}(2 q-1)$ & $-\frac{1}{3}(q+1)$ & $\frac{1}{3}(2 q-1)$ & $-\frac{1}{3}(q+1)$ \\
\hline$\chi_{9}$ & $\frac{1}{3}(q-1)\left(q^{2}-q+1\right)$ & $\frac{1}{3}(2 q-1)$ & $-\frac{1}{3}(q+1)$ & $-\frac{1}{3}(q+1)$ & $\frac{1}{3}(2 q-1)$ \\
\hline$\chi_{10}(n, m)$ & $(q-1)\left(q^{2}-q+1\right)$ & $2 q-1$ & -1 & -1 & -1 \\
\hline$\chi_{11}(n)$ & $(q-1)(q+1)^{2}$ & $-(q+1)$ & -1 & -1 & -1 \\
\hline
\end{tabular}

\begin{tabular}{|c|c|c|}
\hline $\mathrm{PSU}_{3}(q)$ & $C_{6}(a)$ & $C_{7}(a)$ \\
\hline$\chi_{1}$ & 1 & 1 \\
\hline$\chi_{2}$ & $-(q-1)$ & 0 \\
\hline$\chi_{3}$ & $q$ & $\xi_{1}^{3 a n}+\xi_{1}^{-6 a n}$ \\
\hline$\chi_{4}(n)$ & $-q \xi_{1}^{3 a n}+\xi_{1}^{3 a n}+\xi_{1}^{-6 a n}$ & $-\xi_{1}^{3 a n}$ \\
\hline$\chi_{5}(n)$ & $\xi_{1}^{3 a n}-\xi_{1}^{3 a n}+q \xi_{1}^{-6 a n}$ & $\xi_{1}^{3 a n}$ \\
\hline$\chi_{6}(n)$ & $(q+1) \xi_{1}^{3 a n}$ & -1 \\
\hline$\chi_{7}$ & $q-1$ & -1 \\
\hline$\chi_{8}$ & $q-1$ & -1 \\
\hline$\chi_{9}$ & $q-1$ & $-\xi_{1}^{-3 a(-n+m)}-\xi_{1}^{3 a(-2 n+m)}-\xi_{1}^{3 a n}$ \\
\hline$\chi_{10}(n, m)$ & $(q-1)\left(\xi_{1}^{3 a(n-m)}+\xi_{1}^{3 a(-2 n+m)}+\xi_{1}^{3 a n}\right)$ & 0 \\
\hline$\chi_{11}(n)$ & 0 & \\
\hline
\end{tabular}

\begin{tabular}{|c|c|c|}
\hline $\mathrm{PSU}_{3}(q)$ & $C_{8}$ & $C_{9}(a, b)$ \\
\hline$\chi_{1}$ & 1 & 1 \\
\hline$\chi_{2}$ & 2 & 2 \\
\hline$\chi_{3}$ & -1 & -1 \\
\hline$\chi_{4}(n)$ & 3 & $\xi_{1}^{3 a n}+\xi_{1}^{3 b n}+\xi_{1}^{-3 n(a+b)}$ \\
\hline$\chi_{5}(n)$ & -3 & $-\xi_{1}^{3 a n}-\xi_{1}^{3 b n}-\xi_{1}^{-3 n(a+b)}$ \\
\hline$\chi_{6}(n)$ & 0 & 0 \\
\hline$\chi_{7}$ & $-\epsilon_{3}^{\frac{1}{3}(q+1)}-\epsilon_{3}^{\frac{2}{3}(q+1)}$ & $-\epsilon_{3}^{a-b}-\epsilon_{3}^{b-a}$ \\
\hline$\chi_{8}$ & $-\epsilon_{3}^{\frac{1}{3}(q+1)}-\epsilon_{3}^{\frac{2}{3}(q+1)}$ & $-\epsilon_{3}^{a-b}-\epsilon_{3}^{b-a}$ \\
\hline$\chi_{9}$ & $-\epsilon_{3}^{\frac{1}{3}(q+1)}-\epsilon_{3}^{\frac{2}{3}(q+1)}$ & $-\epsilon_{3}^{a-b}-\epsilon_{3}^{b-a}$ \\
\hline$\chi_{10}(n, m)$ & $3 \epsilon_{3}^{m}+3 \epsilon_{3}^{2 m}$ & $-\xi_{1}^{-2 a m-b m+3 a n}-\xi_{1}^{-a m-2 b m+3 b n}-\xi_{1}^{2 a m-3 a n-3 b n+b m}$ \\
\hline$\chi_{11}(n)$ & 0 & $-\xi_{1}^{2 b m-3 a n-3 b n+a m}-\xi_{1}^{a m+3 b n-b m}-\xi_{1}^{b m+3 a n-a m}$ \\
\hline
\end{tabular}




\begin{tabular}{|c|c|c|}
\hline $\mathrm{PSU}_{3}(q)$ & $C_{10}(a)$ & $C_{11}(a)$ \\
\hline$\chi_{1}$ & 1 & 1 \\
\hline$\chi_{2}$ & 0 & -1 \\
\hline$\chi_{3}$ & 1 & -1 \\
\hline$\chi_{4}(n)$ & $\xi_{1}^{3 a n}$ & 0 \\
\hline$\chi_{5}(n)$ & $\xi_{1}^{3 a n}$ & 0 \\
\hline$\chi_{6}(n)$ & $\zeta_{2}^{3 a n}+\zeta_{2}^{-3 q a n}$ & 0 \\
\hline$\chi_{7}$ & 0 & 0 \\
\hline$\chi_{8}$ & 0 & 0 \\
\hline$\chi_{9}$ & 0 & 0 \\
\hline$\chi_{10}(n, m)$ & 0 & $-\phi_{6}^{3 a n}-\phi_{6}^{-3 q a n}-\phi_{6}^{3 q^{2} a n}$ \\
\hline$\chi_{11}(n)$ & 0 & \\
\hline
\end{tabular}

\section{A.2. Computations}

In this section, we provide the detailed computations that we performed in determining which groups of Lie type could occur as a composition factor of a quadratic rational group.

\section{A.2.1. $\operatorname{PSL}_{n}(q)$}

First, assume $n>2$ and $q \geq n$. From 3.1.7, we see that

$$
\phi\left(\frac{q^{n}-1}{d(q-1)}\right) \geq \sqrt{\frac{q^{n}-1}{2 d(q-1)}} \geq \sqrt{\frac{q^{n}-1}{2(q-1)^{2}}}
$$

and $4 d f n \leq 4(q-1) q^{2}$. Using a computer, we see that

$$
\sqrt{\frac{q^{n}-1}{2(q-1)^{2}}}>4(q-1) q^{2}
$$

for all $q \geq n \geq 10$. Thus, $\operatorname{PSL}_{n}(q)$ cannot occur as a composition factor of a quadratic rational group for $q \geq n \geq 10$. Using the same relations $\sqrt{\frac{q^{n}-1}{2 d(q-1)}} \geq \sqrt{\frac{q^{n}-1}{2 n(q-1)}}$ and $4 n d f \leq 4 n^{2} q$, we form a table with the following possibilities for $q$ given $4 \leq n \leq 9$ :

\begin{tabular}{|l|l|l|l|l|l|l|}
\hline $\mathrm{n}$ & 4 & 5 & 6 & 7 & 8 & 9 \\
\hline $\mathrm{q}$ & $4 \leq q \leq 32767$ & $5 \leq q \leq 316$ & $6 \leq q \leq 63$ & $7 \leq q \leq 27$ & $8 \leq q \leq 13$ & $9 \leq q \leq 11$ \\
\hline
\end{tabular}


Using these bounds on $q$, we obtain bounds on $f$ (e.g. if $q \leq 32767$, then $f \leq 14$ since $2^{14}>32767$.) Solving the relation $\sqrt{\frac{q^{n}-1}{2 n(q-1)}}>4 n d f$ with these bounds on $f$, we obtain new upper bounds on $q$. Repeating this procedure sufficiently many times we obtain the following table:

\begin{tabular}{|l|l|l|l|l|l|l|}
\hline $\mathrm{n}$ & 4 & 5 & 6 & 7 & 8 & 9 \\
\hline $\mathrm{q}$ & $4 \leq q \leq 103$ & $5 \leq q \leq 37$ & $6 \leq q \leq 19$ & $7 \leq q \leq 11$ & $8 \leq q \leq 13$ & $9 \leq q \leq 11$ \\
\hline
\end{tabular}

Checking which pairs satisfy $\frac{\phi\left(\frac{q^{n}-1}{d(q-1)}\right)}{n} \mid 4 d f$, we see that no groups $\operatorname{PSL}_{n}(q)$ can occur as a composition factor of a quadratic rational group for $n \geq 4$ and $q \geq n$.

Assume $q \geq n=3$. Using a computer we obtain an upper bound, $f \leq 9$, by solving $\sqrt{\frac{2^{3 f}-1}{2 \cdot 3\left(2^{f}-1\right)}}>4 \cdot 3^{2} f$. Hence, we obtain a table with the remaining possibilities for $p$ by solving the relation $\sqrt{\frac{p^{3 f}-1}{2 \cdot 3\left(p^{f}-1\right)}}>4 \cdot 3^{2} f$ :

\begin{tabular}{|l|l|l|l|l|l|l|l|l|l|}
\hline $\mathrm{f}$ & 1 & 2 & 3 & 4 & 5 & 6 & 7 & 8 & 9 \\
\hline $\mathrm{p}$ & $3 \leq p \leq 83$ & $2 \leq p \leq 13$ & $2 \leq p \leq 5$ & $2 \leq p \leq 3$ & $2 \leq p \leq 3$ & 2 & 2 & 2 & 2 \\
\hline
\end{tabular}

Checking these finitely many cases to see which satisfy $\frac{\phi\left(\frac{q^{3}-1}{d(q-1)}\right)}{3} \mid 4 d f$. We find that $\operatorname{PSL}_{3}(q)$ with $q \geq 3$ may occur as a composition factor of a quadratic rational group for $q \in\{3,4,7,16\}$.

Now, we assume $n>2$ and $n \geq q$. We see that

$$
\phi\left(\frac{q^{n}-1}{d(q-1)}\right) \geq \sqrt{\frac{q^{n}-1}{2 d(q-1)}} \geq \sqrt{\frac{q^{n}-1}{2 n(n-1)}}
$$

and $4 d f n \leq 4 n^{3}$. Using a computer, we see that $\sqrt{\frac{q^{n}-1}{2 n(n-1)}}>4 n^{3}$ for all $n \geq q \geq 9$. Thus, $\operatorname{PSL}_{n}(q)$ cannot occur as a composition factor of a quadratic rational group for pairs $(n, q)$ with $n \geq q \geq 9$. As before, we construct a table to represent the remaining possibilities for $n$, given $q \in\{2,3,4,5,7,8\}$, by solving the relation $\sqrt{\frac{q^{n}-1}{2 n(n-1)}}>4 n^{3}$ :

\begin{tabular}{|l|l|l|l|l|l|l|}
\hline $\mathrm{q}$ & 2 & 3 & 4 & 5 & 7 & 8 \\
$\mathrm{n}$ & $3 \leq n \leq 50$ & $3 \leq n \leq 27$ & $4 \leq n \leq 20$ & $5 \leq n \leq 16$ & $7 \leq n \leq 12$ & $8 \leq n \leq 10$ \\
\hline
\end{tabular}


Checking which pairs $(n, q)$ satisfy $\frac{\phi\left(\frac{q^{n}-1}{d(q-1)}\right)}{n} \mid 4 d f$, we see that $\mathrm{PSL}_{3}(2), \mathrm{PSL}_{3}(3), \mathrm{PSL}_{4}(2)$, and $\mathrm{PSL}_{4}(3)$ can occur as composition factors of a quadratic rational group.

Finally, assume $n=2$. We see that

$$
\phi\left(\frac{q^{2}-1}{d(q-1)}\right) \geq \sqrt{\frac{q+1}{4}}=\sqrt{\frac{p^{f}+1}{4}},
$$

and $2 n d f \leq 8 f$. Using a computer, we see that $\sqrt{\frac{2^{f}+1}{4}}>8 f$ for all $f \geq 16$, thus giving us an upper bound on $f$. As before, we give a table listing the possibilities for $p$, given $1 \leq f \leq 16$, by solving the relation $\sqrt{\frac{p^{f}+1}{4}}>8 f$ :

\begin{tabular}{|l|l|l|l|l|l|l|l|}
\hline $\mathrm{f}$ & 1 & 2 & 3 & 4 & 5 & $6 \leq f \leq 8$ & $9 \leq f \leq 16$ \\
\hline $\mathrm{p}$ & $5 \leq p \leq 253$ & $2 \leq p \leq 31$ & $2 \leq p \leq 13$ & $2 \leq p \leq 7$ & $2 \leq p \leq 5$ & $2 \leq p \leq 3$ & 2 \\
\hline
\end{tabular}

Checking which satisfy $\frac{\phi\left(\frac{q^{2}-1}{d(q-1)}\right)}{2} \mid 2 d f$, we see that $\operatorname{PSL}_{2}(q)$ can occur as a composition factor of a quadratic rational group for $q \in\{4,5,7,8,9,11,16,19,27,31\}$.

Therefore, we have shown that $\operatorname{PSL}_{n}(q)$ can occur as a composition factor of a quadratic rational group for pairs

$$
\begin{gathered}
(n, q) \in\{(2,4),(2,5),(2,7),(2,8),(2,9),(2,11),(2,16),(2,19),(2,23),(2,27),(2,31), \\
(3,2),(3,3),(3,4),(3,7),(3,16),(4,2),(4,3)\} .
\end{gathered}
$$

\section{A.2.2. $\operatorname{PSU}_{n}(q)$}

Recall that we wish to determine when

$$
\frac{\phi\left(\frac{q^{n-1}+1}{(n, q+1)}\right)}{n-1}>2|\operatorname{Out}(S)|=2(n, q+1) f,
$$

where $q^{2}=p^{f}$ and $n$ is even. 
First, assume $n>2$ is even, and $q \geq n$. Let $d=(n, q+1)$. From 3.1.7, we see that

$$
\phi\left(\frac{q^{n-1}+1}{d}\right) \geq \sqrt{\frac{q^{n-1}+1}{2 d}} \geq \sqrt{\frac{q^{n-1}+1}{2(q+1)}},
$$

and $2 d f(n-1) \leq 4(q+1) q(q-1)$. Using a computer, we see that

$$
\sqrt{\frac{q^{n-1}+1}{2(q+1)}}>4(q+1) q(q-1)
$$

for all $q \geq n \geq 10$. Thus, $\operatorname{PSU}_{n}(q)$ cannot occur as a composition factor of a quadratic rational group for $q \geq n \geq 10$. Using the same relations $\sqrt{\frac{q^{n-1}+1}{2 d}} \geq \sqrt{\frac{q^{n-1}+1}{2 n}}$ and $2(n-1) d f \leq 4 n(n-1) q$, we form a table with the following possibilities for $q$ given $4 \leq n \leq 8$

\begin{tabular}{|l|l|l|l|}
\hline $\mathrm{n}$ & 4 & 6 & 8 \\
\hline $\mathrm{q}$ & $4 \leq q \leq 32767$ & $7 \leq q \leq 61$ & $8 \leq q \leq 16$ \\
\hline
\end{tabular}

Using these bounds on $q$, we obtain bounds on $f$ (e.g. if $q \leq 32767$, then $f \leq 30$ since $2^{30}>(32767)^{2}$.) Solving the relation $\sqrt{\frac{q^{n-1}+1}{2 n}}>2(n-1) d f$ with these bounds on $f$, we obtain new upper bounds on $q$. Repeating this procedure sufficiently many times we obtain the following table:

\begin{tabular}{|l|l|l|l|}
\hline $\mathrm{n}$ & 4 & 6 & 8 \\
\hline $\mathrm{q}$ & $4 \leq q \leq 91$ & $7 \leq q \leq 19$ & $8 \leq q \leq 9$ \\
\hline
\end{tabular}

Checking which pairs satisfy $\frac{\phi\left(\frac{q^{n-1}+1}{d}\right)}{n-1} \mid 2 d f$, we see that $\operatorname{PSU}_{n}(q)(q \geq n \geq 4, n$ even) cannot occur as a composition factor of a quadratic rational group.

Now, we assume $n \geq 4$ and $n \geq q, n$ even. We see that

$$
\phi\left(\frac{q^{n-1}+1}{d}\right) \geq \sqrt{\frac{q^{n-1}+1}{2 d}} \geq \sqrt{\frac{q^{n-1}+1}{2 n}},
$$

and $2 d f(n-1) \leq 4 n^{3}$. Using a computer, we see that $\sqrt{\frac{q^{n-1}+1}{2 n}}>4 n^{3}$ for all $n \geq q \geq 9$. Thus, $\operatorname{PSU}_{n}(q)$ cannot occur as a composition factor of a quadratic rational group 
for pairs $(n, q)$ with $n \geq q \geq 9, n$ even. As before, we construct a table to represent the remaining possibilities for $n$, given $q \in\{2,3,4,5,7,8\}$, by solving the relation $\sqrt{\frac{q^{n-1}+1}{2 n}}>4 n^{3}$ :

\begin{tabular}{|l|l|l|l|l|l|l|}
\hline $\mathrm{q}$ & 2 & 3 & 4 & 5 & 7 & 8 \\
\hline $\mathrm{n}$ & $4 \leq n \leq 44$ & $4 \leq n \leq 24$ & $4 \leq n \leq 18$ & $6 \leq n \leq 14$ & $8 \leq n \leq 10$ & $8 \leq n \leq 10$ \\
\hline
\end{tabular}

Checking which pairs $(n, q)$ satisfy $\frac{\phi\left(\frac{q^{n-1}+1}{d}\right)}{n-1} \mid 2 d f$, we see that $\operatorname{PSU}_{n}(q)(n \geq q \geq$ 2, $n$ even) can occur as composition factors of a quadratic rational group for pairs

$$
(n, q) \in\{(4,2),(4,3),(6,2)\}
$$

When $n$ is odd, we wish to determine when

$$
\frac{\phi\left(\frac{q^{n}+1}{(q+1)(n, q+1)}\right)}{n}>2|\operatorname{Out}(S)|=2(n, q+1) f
$$

where $q^{2}=p^{f}$.

First, assume $n>2$ is odd, and $q \geq n$. Let $d=(n, q+1)$. From 3.1.7, we see that

$$
\phi\left(\frac{q^{n}+1}{d(q+1)}\right) \geq \sqrt{\frac{q^{n}+1}{2 d(q+1)}} \geq \sqrt{\frac{q^{n}+1}{2(q+1)^{2}}},
$$

and $2 d f n \leq 4(q+1) q^{2}$. Using a computer, we see that

$$
\sqrt{\frac{q^{n}+1}{2(q+1)^{2}}}>4(q+1) q^{2}
$$

for all $q \geq n \geq 10$. Thus, $\operatorname{PSU}_{n}(q)$ cannot occur as a composition factor of a quadratic rational group for $q \geq n \geq 10$. Using the same relations $\sqrt{\frac{q^{n}+1}{2 d(q+1)}} \geq \sqrt{\frac{q^{n}+1}{2 n(q+1)}}$ and $2 n d f \leq 4 n^{2} q$, we form a table with the following possibilities for $q$ given $4 \leq n \leq 9$ :

\begin{tabular}{|l|l|l|l|}
\hline $\mathrm{n}$ & 5 & 7 & 9 \\
\hline $\mathrm{q}$ & $5 \leq q \leq 316$ & $7 \leq q \leq 27$ & $9 \leq q \leq 11$ \\
\hline
\end{tabular}


Using these bounds on $q$, we obtain bounds on $f$ (e.g. if $q \leq 32767$, then $f \leq 30$ since $2^{30}>(32767)^{2}$.) Solving the relation $\sqrt{\frac{q^{n}+1}{2 n(q+1)}}>2 n d f$ with these bounds on $f$, we obtain new upper bounds on $q$. Repeating this procedure sufficiently many times we obtain the following table:

\begin{tabular}{|l|l|l|l|}
\hline $\mathrm{n}$ & 5 & 7 & 9 \\
\hline $\mathrm{q}$ & $5 \leq q \leq 37$ & $7 \leq q \leq 11$ & $9 \leq q \leq 11$ \\
\hline
\end{tabular}

Checking which pairs satisfy $\frac{\phi\left(\frac{q^{n}+1}{d(q+1)}\right)}{n} \mid 2 d f$, we see that $\operatorname{PSU}_{n}(q)(q \geq n \geq 5, n$ odd) cannot occur as a composition factor of a quadratic rational group.

Assume $q \geq n=3$. Using a computer we obtain an upper bound, $f \leq 20$, by solving $\sqrt{\frac{2^{\frac{3 f}{2}}+1}{2 \cdot 3\left(2^{\frac{f}{2}}+1\right)}}>2 \cdot 3^{2} f$. Hence, we obtain a table with the remaining possibilities for $p$ by solving the relation $\sqrt{\frac{p^{\frac{3 f}{2}}+1}{2 \cdot 3\left(p^{\frac{f}{2}}-1\right)}}>2 \cdot 3^{2} f$ :

\begin{tabular}{|l|l|l|l|l|l|l|}
\hline $\mathrm{f}$ & 2 & 4 & 6 & 8 & 10 & 12 \\
\hline $\mathrm{p}$ & $2 \leq p \leq 173$ & $2 \leq p \leq 17$ & $2 \leq p \leq 7$ & $2 \leq p \leq 5$ & $2 \leq p \leq 3$ & $2 \leq p \leq 3$ \\
\hline
\end{tabular}

\begin{tabular}{|l|l|l|l|l|}
\hline $\mathrm{f}$ & 14 & 16 & 18 & 20 \\
\hline $\mathrm{p}$ & 2 & 2 & 2 & 2 \\
\hline
\end{tabular}

Checking these finitely many cases to see which satisfy $\frac{\phi\left(\frac{q^{3}+1}{d(q+1)}\right)}{3} \mid 2 d f$. We find that $\operatorname{PSU}_{3}(q)$ with $q \geq 3$ may occur as a composition factor of a quadratic rational group for $q \in\{3,4,5,8,11\}$.

Now, we assume $n>2$ and $n \geq q, n$ odd. We see that

$$
\phi\left(\frac{q^{n}+1}{d(q+1)}\right) \geq \sqrt{\frac{q^{n}+1}{2 d(q+1)}} \geq \sqrt{\frac{q^{n}-1}{2 n(n+1)}}
$$

and $2 d f n \leq 4 n^{3}$. Using a computer, we see that $\sqrt{\frac{q^{n}-1}{2 n(n+1)}}>4 n^{3}$ for all $n \geq q \geq 9$. Thus, $\operatorname{PSU}_{n}(q)$ cannot occur as a composition factor of a quadratic rational group for 
pairs $(n, q)$ with $n \geq q \geq 9$. As before, we construct a table to represent the remaining possibilities for $n$, given $q \in\{2,3,4,5,7,8\}$, by solving the relation $\sqrt{\frac{q^{n}+1}{2 n(n+1)}}>4 n^{3}$ :

\begin{tabular}{|l|l|l|l|l|l|l|}
\hline $\mathrm{q}$ & 2 & 3 & 4 & 5 & 7 & 8 \\
\hline $\mathrm{n}$ & $5 \leq n \leq 49$ & $3 \leq n \leq 27$ & $5 \leq n \leq 19$ & $5 \leq n \leq 17$ & $7 \leq n \leq 11$ & 9 \\
\hline
\end{tabular}

Checking which pairs $(n, q)$ satisfy $\frac{\phi\left(\frac{q^{n}+1}{d(q+1)}\right)}{n} \mid 2 d f$, we see that $\operatorname{PSU}_{n}(q)(n \geq q \geq 2$, $n$ odd) can occur as composition factors of a quadratic rational group for pairs

$$
(n, q) \in\{(3,3),(5,2),(5,4),(9,2)\}
$$

Therefore, we find that $\operatorname{PSU}_{n}(q)$ can occur as a composition factor of a quadratic rational group for pairs

$$
(n, q) \in\{(3,3),(3,4),(3,5),(3,8),(3,11),(4,2),(4,3),(5,2),(5,4),(6,2),(9,2)\} .
$$

\section{A.2.3. $\operatorname{PSp}_{2 n}(q)$ and $\mathrm{O}_{2 n+1}(q)$}

Recall that we wish to determine when $\frac{\phi\left(\frac{q^{n}+1}{(2, q-1)}\right)}{2 n}>2\left|\operatorname{Out}\left(\operatorname{PSp}_{2 n}(q)\right)\right|=2 f(2, q-1)$, where $p^{f}=q$.

First, assume $n>2$ and $q \geq n$. From 3.1.7, we see that

$$
\phi\left(\frac{q^{n}+1}{(2, q-1)}\right) \geq \sqrt{\frac{q^{n}+1}{4}}
$$

and $2 f(2, q-1) \cdot 2 n \leq 8 q^{2}$. Using a computer, we see that

$$
\sqrt{\frac{q^{n}+1}{4}}>8 q^{2}
$$

for all $q \geq n \geq 7$. Thus, $\operatorname{PSp}_{2 n}(q)$ cannot occur as a composition factor of a quadratic rational group for $q \geq n \geq 7$. Using the same relations $\phi\left(\frac{q^{n}+1}{(2, q-1)}\right) \geq \sqrt{\frac{q^{n}+1}{4}}$ and $2 f(2, q-1) \cdot 2 n \leq 8 n q$, we form a table with the following possibilities for $q$ given $3 \leq n \leq 6$ 


\begin{tabular}{|l|l|l|l|l|}
\hline $\mathrm{n}$ & 3 & 4 & 5 & 6 \\
\hline $\mathrm{q}$ & $3 \leq q \leq 2304$ & $4 \leq q \leq 64$ & $5 \leq q \leq 18$ & $6 \leq q \leq 16$ \\
\hline
\end{tabular}

Using these bounds on $q$, we obtain bounds on $f$ (e.g. if $q \leq 2304$, then $f \leq 12$ since $2^{12}>2304$.) Solving the relation $\sqrt{\frac{q^{n}+1}{4}}>8 n f$ with these bounds on $f$, we obtain new upper bounds on $q$. Repeating this procedure sufficiently many times we obtain the following table:

\begin{tabular}{|l|l|l|l|l|}
\hline $\mathrm{n}$ & 3 & 4 & 5 & 6 \\
\hline $\mathrm{q}$ & $3 \leq q \leq 37$ & $4 \leq q \leq 16$ & $5 \leq q \leq 8$ & $6 \leq q \leq 7$ \\
\hline
\end{tabular}

Checking which pairs $(n, q)$ satisfy $\frac{\phi\left(\frac{q^{n}+1}{(2, q-1)}\right)}{2 n} \mid 2 f(2, q-1)$, we see that only $\operatorname{PSp}_{6}(3)$ can occur as a composition factor of a quadratic rational group for $q \geq n \geq 3$.

Now, we assume $n>2$ and $n \geq q$. We see that

$$
\phi\left(\frac{q^{n}+1}{(2, q-1)}\right) \geq \sqrt{\frac{q^{n}+1}{4}},
$$

and $2 n \cdot 2 f(2, q-1) \leq 8 n^{2}$. Using a computer, we see that $\sqrt{\frac{q^{n}+1}{4}}>8 n^{2}$ for all $n \geq q \geq 7$. Thus, $\operatorname{PSp}_{2 n}(q)$ cannot occur as a composition factor of a quadratic rational group for pairs $(n, q)$ with $n \geq q \geq 7$. As before, we construct a table to represent the remaining possibilities for $n$, given $q \in\{2,3,4,5\}$, by solving the relation $\sqrt{\frac{q^{n}+1}{4}}>8 n^{2}$ :

\begin{tabular}{|l|l|l|l|l|}
\hline $\mathrm{q}$ & 2 & 3 & 4 & 5 \\
\hline $\mathrm{n}$ & $3 \leq n \leq 27$ & $3 \leq n \leq 14$ & $4 \leq n \leq 10$ & $5 \leq n \leq 8$ \\
\hline
\end{tabular}

Checking which pairs $(n, q)$ satisfy $\frac{\phi\left(\frac{q^{n}+1}{(2, q-1)}\right)}{2 n} \mid 2 f(2, q-1)$, we see that $\operatorname{PSp}_{2 n}(q)$ (where $n \geq q \geq 2, n>2$ ) can occur as composition factors of a quadratic rational 
group for pairs $(n, q) \in\{(3,2),(3,3),(4,2),(5,2)\}$.

Finally, assume $n=2$. We see that

$$
\phi\left(\frac{q^{2}+1}{(2, q-1)}\right) \geq \sqrt{\frac{q^{2}+1}{4}}=\sqrt{\frac{p^{2 f}+1}{4}},
$$

and $2 n \cdot 2 f(2, q-1) \leq 16 f$. Using a computer, we see that $\sqrt{\frac{2^{2 f}+1}{4}}>16 f$ for all $f \geq 9$, thus giving us an upper bound on $f$. As before, we give a table listing the possibilities for $p$, given $1 \leq f \leq 8$, by solving the relation $\sqrt{\frac{p^{2 f}+1}{4}}>16 f$ :

\begin{tabular}{|l|l|l|l|l|l|l|l|l|}
\hline $\mathrm{f}$ & 1 & 2 & 3 & 4 & 5 & 6 & 7 & 8 \\
\hline $\mathrm{p}$ & $2 \leq p \leq 31$ & $2 \leq p \leq 7$ & $2 \leq p \leq 3$ & $2 \leq p \leq 3$ & 2 & 2 & 2 & 2 \\
\hline
\end{tabular}

Checking which satisfy $\frac{\phi\left(\frac{q^{2}+1}{(2, q-1)}\right)}{4} \mid 2 f(2, q-1)$, we see that $\operatorname{PSp}_{4}(2)^{\prime}$ and $\operatorname{PSp}_{4}(q)$ can occur as a composition factor of a quadratic rational group for $q \in\{3,4\}$.

Therefore, we have found that $\operatorname{PSp}_{2 n}(q)$ can occur as a composition factor of a quadratic rational group for pairs

$$
(n, q) \in\{(2,2),(2,3),(2,4),(3,2),(3,3),(4,2),(5,2)\}
$$

\section{A.2.4. $\mathrm{O}_{2 n}^{+}(q)$}

Recall that we wish to determine when

$$
\frac{\phi\left(\frac{\left(q^{n-1}+1\right)(q+1)}{\left(q+1, q^{n-1}+1\right)_{2^{\prime}}(q+1)_{2}\left(q^{n-1}+1\right)_{2}}\right)}{2(n-1)}>2\left|\operatorname{Out}\left(O_{2 n}^{+}(q)\right)\right|= \begin{cases}12(2, q-1)^{2} f & n=4 \\ 4(2, q-1)^{2} f & n \geq 6, n \text { even } \\ 4\left(4, q^{n}-1\right) f & n \geq 5, n \text { odd }\end{cases}
$$

where $p^{f}=q$.

First, assume that $n=4$. We see that

$$
\frac{\phi\left(\frac{\left(q^{n-1}+1\right)(q+1)}{\left(q+1, q^{n-1}+1\right)_{2^{\prime}}(q+1)_{2}\left(q^{n-1}+1\right)_{2}}\right)}{2(n-1)}=\frac{\phi\left(\frac{\left(q^{3}+1\right)(q+1)}{\left(q+1, q^{3}+1\right)_{2^{\prime}}(q+1)_{2}\left(q^{3}+1\right)_{2}}\right)}{6}
$$




$$
\geq \sqrt{\frac{\left(q^{3}+1\right)(q+1)}{72\left(q+1, q^{3}+1\right)_{2^{\prime}}(q+1)_{2}\left(q^{3}+1\right)_{2}}} \geq \sqrt{\frac{q^{3}+1}{72(q+1)}}=\sqrt{\frac{q^{2}-q+1}{72}}
$$

and $12(2, q-1)^{2} f=48 f=48 \frac{\ln (q)}{\ln (p)} \leq 48 \ln (q)$ for $q$ odd. Using a computer, we see that $\sqrt{\frac{q^{2}-q+1}{72}}>48 \ln (q)$ for all $q \geq 3300$. Therefore, $O_{8}^{+}(q)$ cannot occur as a composition factor of a quadratic rational group for $q \geq 3300, q$ odd. As $3^{8}=6561$, it follows that $f \leq 7$.

Now, for $1 \leq f \leq 7$, we make a table for the various possible values of $p$, subject to $\sqrt{\frac{q^{2}-q+1}{72}}>48 f$ :

\begin{tabular}{|l|l|l|l|l|l|l|l|}
\hline $\mathrm{f}$ & 1 & 2 & 3 & 4 & 5 & 6 & 7 \\
\hline $\mathrm{p}$ & $3 \leq p \leq 407$ & $3 \leq p \leq 23$ & $3 \leq p \leq 7$ & $3 \leq p \leq 5$ & 3 & 3 & 3 \\
\hline
\end{tabular}

Checking which of these values satisfy the relation

$$
\frac{\phi\left(\frac{\left(q^{3}+1\right)(q+1)}{\left(q+1, q^{3}+1\right)_{2^{\prime}}(q+1)_{2}\left(q^{3}+1\right)_{2}}\right)}{6} \mid 48 f
$$

we see that $O_{8}^{+}(q)$ with $q$ odd can occur as a composition factor of a quadratic rational group only if $q \in\{3,5,9\}$. If $q$ is even, we see that

$$
\frac{\phi\left(\frac{\left(q^{n-1}+1\right)(q+1)}{\left(q+1, q^{n-1}+1\right)_{2^{\prime}}(q+1)_{2}\left(q^{n-1}+1\right)_{2}}\right)}{2(n-1)}=\frac{\phi\left(\frac{\left(q^{n-1}+1\right)(q+1)}{\left(q+1, q^{n-1}+1\right)_{2^{\prime}}}\right)}{2(n-1)} \geq \frac{\sqrt{\frac{q^{3}+1}{2}}}{6}=\frac{\sqrt{\frac{2^{3 f}+1}{2}}}{6}
$$

and $12(2, q-1)^{2} f=12 f$. Using a computer, we see that $\frac{\sqrt{\frac{2^{3 f}+1}{2}}}{6}>12 f$ for all $f \geq 7$. Checking which values $q=2^{f}$ for $1 \leq f \leq 6$ satisfy

$$
\frac{\phi\left(\frac{\left(q^{3}+1\right)(q+1)}{\left(q+1, q^{3}+1\right)_{2^{\prime}}}\right)}{6} \mid 12 f
$$

we see that $O_{8}^{+}(q)$ for $q$ even can occur as a composition factor of a quadratic rational group only if $q \in\{2,4\}$. 
Now, assume that $n \geq 6$ is even. We see that

$$
\phi\left(\frac{\left(q^{n-1}+1\right)(q+1)}{\left(q+1, q^{n-1}+1\right)_{2^{\prime}}(q+1)_{2}\left(q^{n-1}+1\right)_{2}}\right) \geq \sqrt{\frac{\left(q^{n-1}+1\right)(q+1)}{2(q+1)^{2}}}=\sqrt{\frac{q^{n-1}+1}{2(q+1)}},
$$

and $2(n-1) \cdot 4(2, q-1)^{2} f \leq 32(n-1) q$.

First, assume that $n \leq q$. Using a computer, we see that $\sqrt{\frac{q^{n-1}+1}{2(q+1)}}>32 q(q-1)$ for all $10 \leq n \leq q$. Thus, $O_{2 n}^{+}(q)$ cannot occur as a composition factor of a quadratic rational group for $10 \leq n \leq q, n$ even. Now, using the relation

$$
\sqrt{\frac{q^{n-1}+1}{2(q+1)}}>32(n-1) q
$$

with $6 \leq n \leq 8$ even, we obtain the following table of possibilities for values of $q \geq n$ :

\begin{tabular}{|l|l|l|}
\hline $\mathrm{n}$ & 6 & 8 \\
\hline $\mathrm{q}$ & $7 \leq q \leq 223$ & $8 \leq q \leq 17$ \\
\hline
\end{tabular}

Assume $n=6$. Then, $q \leq 223$ tells us that $f \leq 7$ since $2^{8}=256$. Thus, using the relation $\sqrt{\frac{q^{5}+1}{2(q+1)}}>160 f$, we obtain a new upper bound for $q$, namely $q \leq 40$. Hence, a new upper bound for $f$ is $f \leq 5$. As before, this yields $q \leq 32$. Checking which values of $7 \leq q \leq 32$ satisfy $\frac{\phi\left(\frac{\left(q^{5}+1\right)(q+1)}{\left(q+1, q^{5}+1\right)_{2^{\prime}}(q+1)_{2}\left(q^{5}+1\right)_{2}}\right)}{10} \mid 4(2, q-1)^{2} f$, we see that $O_{12}^{+}(q)$ cannot occur as a composition factor of a quadratic rational group for $q \geq 7$.

Now, assume $n=8$. Since $q \leq 17$, we obtain an upper bound on $f$, namely $f \leq 4$. Hence, using $\sqrt{\frac{q^{7}+1}{2(q+1)}}>224 f$, we have that $q \leq 9$. Checking which values $8 \leq q \leq 9$ satisfy $\frac{\phi\left(\frac{\left(q^{7}+1\right)(q+1)}{\left(q+1, q^{7}+1\right)_{2^{\prime}}(q+1)_{2}\left(q^{7}+1\right)_{2}}\right)}{10} \mid 4(2, q-1)^{2} f$, we see that $O_{16}^{+}(q)$ cannot occur as a composition factor of a quadratic rational group for $q \geq 8$.

Now, assume that $n \geq q$. Then, using a computer, we see that $\sqrt{\frac{q^{n-1}+1}{2(n+1)}}>$ $32 n(n-1)$ for all $9 \leq q \leq n, n$ even, and $O_{2 n}^{+}(q)$ cannot occur as a composition factor of a quadratic rational group for $9 \leq q \leq n$, $n$ even. Using the relation 
$\sqrt{\frac{q^{n-1}+1}{2(q+1)}}>8(n-1)(2, q-1)^{2} f$ with $2 \leq q \leq 8$, we obtain the following table of possibilities for values of $n \geq q$ (note that $q=7,8$ never satisfy this for $n \geq q, n$ even):

\begin{tabular}{|l|l|l|l|l|}
\hline $\mathrm{q}$ & 2 & 3 & 4 & 5 \\
\hline $\mathrm{n}$ & $6 \leq n \leq 16$ & $6 \leq n \leq 12$ & $6 \leq n \leq 8$ & $6 \leq n \leq 8$ \\
\hline
\end{tabular}

Checking which of these values satisfy $\frac{\phi\left(\frac{\left(q^{n-1}+1\right)(q+1)}{\left(q+1, q^{n-1}+1\right)_{2}(q+1)_{2}\left(q^{n-1}+1\right)_{2}}\right)}{2(n-1)} \mid 4(2, q-1)^{2} f$, we see that only $O_{12}^{+}(2)$ can occur as a composition factor of a quadratic rational group.

Lastly, assume $n \geq 5$ is odd. We see that

$$
\phi\left(\frac{\left(q^{n-1}+1\right)(q+1)}{\left(q+1, q^{n-1}+1\right)_{2^{\prime}}(q+1)_{2}\left(q^{n-1}+1\right)_{2}}\right) \geq \sqrt{\frac{\left(q^{n-1}+1\right)(q+1)}{2(q+1)^{2}}}=\sqrt{\frac{q^{n-1}+1}{2(q+1)}},
$$

and $2(n-1) \cdot 4\left(4, q^{n}+1\right)^{2} f \leq 32(n-1) q$.

First, assume that $n \leq q$. Using a computer, we see that $\sqrt{\frac{q^{n-1}+1}{2(q+1)}}>32 q(q-1)$ for all $11 \leq n \leq q$. Thus, $O_{2 n}^{+}(q)$ cannot occur as a composition factor of a quadratic rational group for $11 \leq n \leq q, n$ odd. Now, using the relation

$$
\sqrt{\frac{q^{n-1}+1}{2(q+1)}}>32(n-1) q
$$

with $5 \leq n \leq 9$ odd, we obtain the following table of possibilities for values of $q \geq n$ :

\begin{tabular}{|l|l|l|l|}
\hline $\mathrm{n}$ & 5 & 7 & 9 \\
\hline $\mathrm{q}$ & $5 \leq q \leq 32769$ & $7 \leq q \leq 41$ & 9 \\
\hline
\end{tabular}

Assume $n=5$. Then, $q \leq 32769$ tells us that $f \leq 15$ since $2^{16}=65536$. Thus, using the relation $\sqrt{\frac{q^{5}+1}{2(q+1)}}>128 f$, we obtain a new upper bound for $q$, namely $q \leq 49$. Hence, a new upper bound for $f$ is $f \leq 5$. As before, this yields $q \leq 29$. 
Checking which values of $5 \leq q \leq 29$ satisfy $\frac{\phi\left(\frac{\left(q^{4}+1\right)(q+1)}{\left(q+1, q^{4}+1\right)_{2^{\prime}}(q+1)_{2}\left(q^{4}+1\right)_{2}}\right)}{8} \mid 4\left(4, q^{5}+1\right) f$, we see that $O_{10}^{+}(q)$ cannot occur as a composition factor of a quadratic rational group for $q \geq 5$.

Now, assume $n=7$. Since $q \leq 41$, we obtain an upper bound on $f$, namely $f \leq 5$. Hence, using $\sqrt{\frac{q^{7}+1}{2(q+1)}}>192 f$, we have that $q \leq 11$. Checking which values $7 \leq q \leq 11$ satisfy $\frac{\phi\left(\frac{\left(q^{6}+1\right)(q+1)}{\left(q+1, q^{6}+1\right)_{2^{\prime}}(q+1)_{2}\left(q^{6}+1\right)_{2}}\right)}{12} \mid 4\left(4, q^{7}+1\right) f$, we see that $O_{14}^{+}(q)$ cannot occur as a composition factor of a quadratic rational group for $q \geq 7$.

Now, assume that $n \geq q$. Then, using a computer, we see that $\sqrt{\frac{q^{n-1}+1}{2(n+1)}}>$ $32 n(n-1)$ for all $9 \leq q \leq n, n$ even, and $O_{2 n}^{+}(q)$ cannot occur as a composition factor of a quadratic rational group for $9 \leq q \leq n, n$ odd. Using the relation $\sqrt{\frac{q^{n-1}+1}{2(q+1)}}>8(n-1)\left(4, q^{n}-1\right) f$ with $2 \leq q \leq 8$, we obtain the following table of possibilities for values of $n \geq q$ (note that $q=8$ never satisfies this for $n \geq 8, n$ odd):

\begin{tabular}{|l|l|l|l|l|l|}
\hline $\mathrm{q}$ & 2 & 3 & 4 & 5 & 7 \\
\hline $\mathrm{n}$ & $5 \leq n \leq 17$ & $5 \leq n \leq 13$ & $5 \leq n \leq 9$ & $5 \leq n \leq 7$ & 7 \\
\hline
\end{tabular}

Checking which of these values satisfy $\frac{\phi\left(\frac{\left.q^{n-1}+1\right)(q+1)}{\left(q+1, q^{n-1}+1\right)_{2}(q(1))_{2}\left(q^{n-1}+1\right)_{2}}\right)}{2(n-1)} \mid 4\left(4, q^{n}-1\right) f$, we see that only $O_{10}^{+}(2)$ can occur as a composition factor of a quadratic rational group.

\section{A.2.5. $\mathrm{O}_{2 n}^{-}(q)$}

Recall that we wish to determine when $\frac{\phi\left(\frac{q^{n}+1}{\left(q^{n}+1\right)_{2}}\right)}{n}>2\left|\operatorname{Out}\left(O_{2 n}^{-}(q)\right)\right|=2\left(4, q^{n}+1\right) f$, where $p^{f}=q^{2}$.

We see that

$$
\phi\left(\frac{q^{n}+1}{\left(q^{n}+1\right)_{2}}\right) \geq \sqrt{\frac{q^{n}+1}{2\left(q^{n}+1\right)_{2}}} \geq \sqrt{\frac{q^{n}+1}{2(q+1)_{2}}} \geq \sqrt{\frac{q^{n}+1}{2(q+1)}},
$$


and $n \cdot 2\left(4, q^{n}+1\right) f \leq 16 n q$.

First, assume that $n \leq q$. Using a computer, we see that $\sqrt{\frac{q^{n}+1}{2(q+1)}}>16 q^{2}$ for all $9 \leq n \leq q$. Thus, $O_{2 n}^{-}(q)$ cannot occur as a composition factor of a quadratic rational group for $9 \leq n \leq q$. Now, using the relation

$$
\sqrt{\frac{q^{n}+1}{2(q+1)}}>16 n q
$$

wiith $4 \leq n \leq 8$, we obtain the following table of possibilities for values of $q \geq n$ :

\begin{tabular}{|l|l|l|l|l|l|}
\hline $\mathrm{n}$ & 4 & 5 & 6 & 7 & 8 \\
\hline $\mathrm{q}$ & $4 \leq q \leq 8192$ & $5 \leq q \leq 113$ & $7 \leq q \leq 27$ & $7 \leq q \leq 13$ & 8 \\
\hline
\end{tabular}

Assume $n=4$. Then, $q \leq 8192$ tells us that $f \leq 26$ since $2^{13}=8192$. Thus, using the relation $\sqrt{\frac{q^{4}+1}{2(q+1)}}>32 f$, we obtain a new upper bound for $q$, namely $q \leq 111$. Hence, a new upper bound for $f$ is $f \leq 12$. As before, this yields $q \leq 64$. Checking which values of $4 \leq q \leq 64$ satisfy $\frac{\phi\left(\frac{q^{4}+1}{\left(q^{4}+1\right)_{2}}\right)}{4} \mid 2\left(4, q^{4}+1\right) f$, we see that $O_{8}^{-}(q)$ cannot occur as a composition factor of a quadratic rational group for $q \geq 4$.

Now, assume $n=5$. Since $q \leq 113$, we obtain an upper bound on $f$, namely $f \leq$ 12. Hence, using $\sqrt{\frac{q^{5}+1}{2(q+1)}}>40 f$, we have that $q \leq 25$. As before, this yields $f \leq 8$, and thus $q \leq 19$. Checking which values $5 \leq q \leq 19$ satisfy $\frac{\phi\left(\frac{q^{5}+1}{\left(q^{5}+1\right)_{2}}\right)}{5} \mid 2\left(4, q^{5}+1\right) f$, we see that $O_{10}^{-}(q)$ cannot occur as a composition factor of a quadratic rational group for $q \geq 5$.

Lastly, checking which of the remaining cases $(n=6,7,8)$ satisfy $\frac{\phi\left(\frac{q^{n}+1}{\left(q^{n}+1\right)_{2}}\right)}{n}$ | $2\left(4, q^{n}+1\right) f$, we see that $O_{2 n}^{-}(q)$ cannot occur as a composition factor of a quadratic rational group for $n \leq q$.

Now, assume that $n \geq q$. Then, using a computer, we see that $\sqrt{\frac{q^{n}+1}{2(n+1)}}>16 n^{2}$ for all $9 \leq q \leq n$, and $O_{2 n}^{-}(q)$ cannot occur as a composition factor of a quadratic rational 
group for $9 \leq q \leq n$. Using the relation $\sqrt{\frac{q^{n}+1}{2(q+1)_{2}}}>8 n f$ with $2 \leq q \leq 9$, we obtain the following table of possibilities for values of $n \geq q$ (note that no values of $n \geq q$ satisfy this relation for $q \geq 7$ ):

\begin{tabular}{|l|l|l|l|l|}
\hline $\mathrm{q}$ & 2 & 3 & 4 & 5 \\
\hline $\mathrm{n}$ & $4 \leq n \leq 17$ & $4 \leq n \leq 11$ & $4 \leq n \leq 8$ & $5 \leq n \leq 6$ \\
\hline
\end{tabular}

Checking which of these values satisfy $\frac{\phi\left(\frac{q^{n}+1}{\left(q^{n}+1\right)_{2}}\right)}{n} \mid 2\left(4, q^{n}+1\right) f$, we see that $O_{2 n}^{-}(q)$ with $q \leq n$ can occur as a composition factor of a quadratic rational group only for pairs

$$
(n, q) \in\{(4,2),(5,2)\}
$$

\section{A.2.6. ${ }^{2} \mathrm{~B}_{2}(q)$}

Recall that we wish to determine when

$$
\frac{\phi(q \pm \sqrt{2 q}+1)}{4}>2|\operatorname{Out}(S)|=2(2 n+1),
$$

where $q=2^{2 n+1}$.

We see that

$$
\phi(q+\sqrt{2 q}+1) \geq \sqrt{\frac{q+\sqrt{2 q}+1}{2}}=\sqrt{\frac{2^{2 n+1}+2^{n+1}+1}{2}},
$$

and $4 \cdot 2(2 n+1)=8(2 n+1)$. Using a computer, we find that $\sqrt{\frac{2^{2 n+1}+2^{n+1}+1}{2}}>$ $8(2 n+1)$ for all $n \geq 7$, and ${ }^{2} B_{2}(q)$ cannot occur as a composition factor of a quadratic rational group for $q=2^{2 n+1} \geq 2^{7}$.

Checking which of the remaining six possibilities satisfy $\frac{\phi(q+\sqrt{2 q}+1)}{4} \mid 2(2 n+$ 1 ), we see that ${ }^{2} B_{2}(8)$ and ${ }^{2} B_{2}(32)$ can occur as composition factors of a quadratic rational group. 


\section{A.2.7. ${ }^{3} \mathrm{D}_{4}(q)$}

Recall that we wish to determine when

$$
\frac{\phi\left(q^{4}-q^{2}+1\right)}{4}>2|\operatorname{Out}(S)|=2 f
$$

where $q^{3}=p^{f}$.

We have that

$$
\phi\left(q^{4}-q^{2}+1\right) \geq \sqrt{\frac{q^{4}-q^{2}+1}{2}},
$$

and $4 \cdot 2 f \leq 24 q$. Using a computer, we see that $\sqrt{\frac{q^{4}-q^{2}+1}{2}}>24 q$ for all $q \geq 34$. Thus, as $2^{15}>34^{3}$, we have $f \leq 15$. Using this bound on $f$, we see that $\sqrt{\frac{q^{4}-q^{2}+1}{2}}>120$ for all $q \geq 14$. Repeating this process, we achieve a new upper bound on $f$, namely $f \leq 9$, which gives a new upper bound on $q$, namely $q \leq 9$. Checking which values $2 \leq q \leq 9$ satisfy $\frac{\phi\left(q^{4}-q^{2}+1\right)}{4} \mid 2 f$, where $q^{3}=p^{f}$, we see that only ${ }^{3} D_{4}(2)$ can occur as a composition factor of a quadratic rational group.

\section{A.2.8. $\quad \mathrm{E}_{6}(q)$}

Recall that we wish to determine when

$$
\frac{\phi\left(\frac{q^{6}+q^{3}+1}{(3, q-1)}\right)}{9}>2|\operatorname{Out}(S)|=4(3, q-1) f
$$

where $q=p^{f}$.

We have that $\phi\left(\frac{q^{6}+q^{3}+1}{(3, q-1)}\right) \geq \sqrt{\frac{q^{6}+q^{3}+1}{6}}$, and $9 \cdot 4(3, q-1) f \leq 108 q$. Using a computer, we see that $\sqrt{\frac{q^{6}+q^{3}+1}{6}}>108 q$ for all $q \geq 17$, and $E_{6}(q)$ cannot occur as a composition factor of a quadratic rational group for $q \geq 17$. Checking which of the remaining values of $p^{f}=q \leq 16$ satisfy $\frac{\phi\left(\frac{q^{6}+q^{3}+1}{(3, q-1)}\right)}{9} \mid 4(3, q-1) f$, we see that $E_{6}(q)$ cannot occur as a composition factor of a quadratic rational group for any $q$. 


\section{A.2.9. ${ }^{2} \mathrm{E}_{6}(q)$}

Recall that we wish to determine when

$$
\frac{\phi\left(\frac{q^{6}-q^{3}+1}{(3, q+1)}\right)}{9}>2|\operatorname{Out}(S)|=2(3, q+1) f
$$

where $q^{2}=p^{f}$.

We see that $\phi\left(\frac{q^{6}-q^{3}+1}{(3, q+1)}\right) \geq \sqrt{\frac{q^{6}-q^{3}+1}{6}}$, and $9 \cdot 2(3, q+1) f \leq 108 q$. Using a computer, we see that $\sqrt{\frac{q^{6}-q^{3}+1}{6}}>108 q$ for all $q \geq 17$, and ${ }^{2} E_{6}(q)$ cannot occur as a composition factor of a quadratic rational group for $q \geq 17$.

Checking which of the remaining possibilities of $q=p^{f / 2}$ satisfy $\frac{\phi\left(\frac{q^{6}-q^{3}+1}{(3, q+1)}\right)}{9} \mid 2(3, q+$ 1) $f$, we see that only ${ }^{2} E_{6}(2)$ can occur as a composition factor of a quadratic rational group.

\section{A.2.10. $\quad \mathrm{E}_{7}(q)$}

Recall that we wish to determine when

$$
\frac{\phi\left(\frac{(q+1)\left(q^{6}-q^{3}+1\right)}{(2, q-1)}\right)}{18}>2|\operatorname{Out}(S)|=2(2, q-1) f
$$

where $q=p^{f}$.

We see that

$$
\phi\left(\frac{(q+1)\left(q^{6}-q^{3}+1\right)}{(2, q-1)} \geq \sqrt{\frac{(q+1)\left(q^{6}-q^{3}+1\right)}{2(2, q-1)}} \geq \sqrt{\frac{(q+1)\left(q^{6}-q^{3}+1\right)}{4}},\right.
$$

and $18 \cdot 2(2, q-1) f \leq 72 q$. Using a computer, we see that $\sqrt{\frac{(q+1)\left(q^{6}-q^{3}+1\right)}{4}}>72 q$ for all $q \geq 8$, and $E_{7}(q)$ cannot occur as a composition factor of a quadratic rational group for $q \geq 8$. Checking which of the remaining five possible cases for $q$ satisfy $\frac{\phi\left(\frac{(q+1)\left(q^{6}-q^{3}+1\right)}{(2, q-1)}\right)}{18} \mid 2(2, q-1) f$, we see that $E_{7}(q)$ cannot occur as a composition factor of any quadratic rational group. 


\section{A.2.11. $\quad \mathrm{E}_{8}(q)$}

Recall that we wish to determine when

$$
\frac{\phi\left(q^{8}+q^{7}-q^{5}-q^{4}-q^{3}+q+1\right)}{30}>2|\operatorname{Out}(S)|=2 f
$$

where $q=p^{f}$.

We see that

$$
\phi\left(q^{8}+q^{7}-q^{5}-q^{4}-q^{3}+q+1\right) \geq \sqrt{\frac{q^{8}+q^{7}-q^{5}-q^{4}-q^{3}+q+1}{2}},
$$

and $30 \cdot 2 f \leq 60 q$. Using a computer, we see that $\sqrt{\frac{q^{8}+q^{7}-q^{5}-q^{4}-q^{3}+q+1}{2}}>60 q$ for all $q \geq 5$. Checking to see if any of the remaining possibilities of $q=p^{f}$ satisfy $\frac{\phi\left(q^{8}+q^{7}-q^{5}-q^{4}-q^{3}+q+1\right)}{30} \mid 2 f$, we see that $E_{8}(q)$ cannot occur as a composition factor of a quadratic rational group for any $q$.

\section{A.2.12. $\quad \mathrm{F}_{4}(q)$}

Recall that we wish to determine when

$$
\frac{\phi\left(q^{4}-q^{2}+1\right)}{12}>2|\operatorname{Out}(S)|=\left\{\begin{array}{ll}
2 f & 2 \nmid q \\
4 f & q=2^{f}
\end{array} .\right.
$$

First, assume $q$ is even. We see that $\phi\left(q^{4}-q^{2}+1\right) \geq \sqrt{\frac{q^{4}-q^{2}+1}{2}}$, and $12 \cdot 4 f \leq 48 q$. Using a computer, we have that $\sqrt{\frac{q^{4}-q^{2}+1}{2}}>48 q$ for all 68 . Checking which of the six remaining values of $q=2^{f} \leq 64$ satisfy $\frac{\phi\left(q^{4}-q^{2}+1\right)}{12} \mid 4 f$, we see that only $F_{4}(2)$ can occur as a composition factor of a quadratic group for $q$ even.

Now, assume $q$ is odd. As before, we have $\phi\left(q^{4}-q^{2}+1\right) \geq \sqrt{\frac{q^{4}-q^{2}+1}{2}}$, and $12 \cdot 2 f \leq 24 q$. Using a computer, we have that $\sqrt{\frac{q^{4}-q^{2}+1}{2}}>24 q$ for all 34 , and $F_{4}(q)$ cannot occur as a composition factor of a quadratic rational group for $q \geq 34$. Checking if any of the remaining values of $q=p^{f} \leq 31$ satisfy $\frac{\phi\left(q^{4}-q^{2}+1\right)}{12} \mid 2 f$, we see that $F_{4}(q)$ cannot occur as a composition factor of a quadratic group for $q$ odd. 
Therefore, we have shown that only $F_{4}(2)$ can occur as a composition factor of a quadratic rational group.

\section{A.2.13. ${ }^{2} \mathrm{~F}_{4}(q)$}

Recall that we wish to determine when

$$
\frac{\phi\left(q^{2} \pm \sqrt{2 q^{3}}+q \pm \sqrt{2 q}+1\right)}{12}>2|\operatorname{Out}(S)|=2(2 n+1),
$$

where $q=2^{2 n+1}$.

We have that $\phi\left(q^{2}+\sqrt{2 q^{3}}+q+\sqrt{2 q}+1\right) \geq \sqrt{\frac{2^{4 n+2}+2^{3 n+2}+2^{2 n+1}+2^{n+1}+1}{2}} \geq$ $\sqrt{\frac{2^{4 n+2}-2^{3 n+2}+2^{2 n+1}-2^{n+1}+1}{2}}$, and $12 \cdot 2(2 n+1)=24(2 n+1)$. Using a computer, we see that $\sqrt{\frac{2^{4 n+2}-2^{3 n+2}+2^{2 n+1}-2^{n+1}+1}{2}}>24(2 n+1)$ for all $n \geq 4$, and ${ }^{2} F_{4}(q)$ cannot occur as a composition factor of a quadratic rational group for $q=2^{2 n+1} \geq 2^{9}$. Checking which of the remaining values of $q=2^{2 n+1}$ satisfy $\frac{\phi\left(q^{2} \pm \sqrt{2 q^{3}}+q \pm \sqrt{2 q}+1\right)}{12}$ | $2(2 n+1)$, we see that only ${ }^{2} F_{4}(2)^{\prime}$ can occur as a composition factor of a quadratic rational group.

\section{A.2.14. $\mathrm{G}_{2}(q)$}

Recall that we wish to determine when

$$
\frac{\phi\left(q^{2}-q+1\right)}{6}>2|\operatorname{Out}(S)|=\left\{\begin{array}{ll}
2 f & q \not \equiv 0 \bmod 3 \\
4 f & q \equiv 0 \bmod 3
\end{array} .\right.
$$

First, assume that $3 \nmid q$. We see that $\phi\left(q^{2}-q+1\right) \geq \sqrt{\frac{q^{2}-q+1}{2}}=\sqrt{\frac{p^{2 f}-p^{f}+1}{2}}$ and $6 \cdot 2 f=12 f$. Using a computer, we obtain an upper bound of $f \leq 6$ since $\sqrt{\frac{22^{2 f}-2^{f}+1}{2}}>$ $12 f$ for all $f \geq 7$. Using the relation $\sqrt{\frac{p^{2 f}-p^{f}+1}{2}}>12 f$, we list the possible values of $p$, given $1 \leq f \leq 6$ :

\begin{tabular}{|l|l|l|l|l|l|l|}
\hline $\mathrm{f}$ & 1 & 2 & 3 & 4 & 5 & 6 \\
\hline $\mathrm{p}$ & $2 \leq p \leq 19$ & $2 \leq p \leq 7$ & $2 \leq p \leq 5$ & 2 & 2 & 2 \\
\hline
\end{tabular}


Checking which of these values of $q=p^{f}$ satisfy $\frac{\phi\left(q^{2}-q+1\right)}{6} \mid 2 f$, we see that $G_{2}(2)^{\prime}, G_{2}(4), G_{2}(8)$, and $G_{2}(5)$ can occur as composition factors of quadratic rational groups.

Now, assume that $q=3^{f}$. Then $\phi\left(q^{2}-q+1\right) \geq \sqrt{\frac{3^{2 f}-3^{f}+1}{2}}$, and $6 \cdot 4 f=24 f$. Using a computer, we see that $\sqrt{\frac{3^{2 f}-3^{f}+1}{2}}>24 f$ for all $f \geq 5$, giving an upper bound on $3^{f}=q \leq 81$. Checking which of these five values of $q$ satisfies $\frac{\phi\left(q^{2}-q+1\right)}{6} \mid 4 f$, we see that only $G_{2}(3)$ can occur as a composition factor of a quadratic rational group.

Therefore, we have shown that only $G_{2}(2)^{\prime}, G_{2}(3), G_{2}(4), G_{2}(8)$, and $G_{2}(5)$ can occur as composition factors of a quadratic rational group.

\section{A.2.15. ${ }^{2} \mathrm{G}_{2}(q)$}

Recall that we wish to determine when

$$
\frac{\phi(q \pm \sqrt{3 q}+1)}{6}>2|\operatorname{Out}(S)|=2(2 n+1),
$$

where $q=3^{2 n+1}$.

We see that

$$
\phi(q+\sqrt{3 q}+1) \geq \sqrt{\frac{q+\sqrt{3 q}+1}{2}} \geq \sqrt{\frac{q-\sqrt{3 q}+1}{2}}=\sqrt{\frac{3^{2 n+1}-3^{n+1}+1}{2}},
$$

and $6 \cdot 2(2 n+1)=12(2 n+1)$. Using a computer, we see that $\sqrt{\frac{3^{2 n+1}-3^{n+1}+1}{2}}>$ $12(2 n+1)$ for all $n \geq 5$, and ${ }^{2} G_{2}(q)$ cannot occur as a composition factor for $q=$ $3^{2 n+1} \geq 3^{11}$.

Checking which of the five values satisfy $\frac{\phi(q \pm \sqrt{3 q}+1)}{6} \mid 2(2 n+1)$, we see that ${ }^{2} G_{2}(3)^{\prime}$ and ${ }^{2} G_{2}(27)$ can occur as composition factors of a quadratic rational group. 


\section{REFERENCES}

[1] E. Artin, The orders of the linear groups, Comm. Pure Appl. Math. 8, 1955, pp. 355-366.

[2] L. Babai, P. P. Pálfy, J. Saxl, On the number of p-regular elements in simple groups, LMS J. Comput. Math. 12 (2009), pp. 82-119.

[3] W. Bosma, J. Cannon, C. Playoust, The Magma algebra system. I. The user language, J. Symbolic Comput., (1997), pp. 235265.

[4] M. Broué, J. Michel, Blocs et séries de Lusztig dans un groupe réductif fini, J. reine angew. Math. 395 (1989), 56-67.

[5] A. A. Buturlakin, M. A. Grechkoseeva, The cyclic structure of maximal tori of the finite classical groups, Algebra and Logic, March 2007, Volume 46, Issue 2, pp. 73-89.

[6] R. Carter, Finite Groups of Lie Type: Conjugacy Classes and Complex Characters, Wiley, Chichester, 1985.

[7] R. Carter, Simple Groups of Lie Type, Wiley, Bartholomew Press, Dorking, 1972.

[8] J. H. Conway, R. T. Curtis, S.P. Norton, R.A. Parker, R.A. Wilson, An ATLAS of Finite Groups, Clarendon Press, Oxford, 1985.

[9] D. I. Deriziotis, A. P. Fakiolas, The maximal tori of the finite Chevalley groups of type $E_{6}, E_{7}$ and $E_{8}$, Comm. Algebra 19 (1991), pp. 889-903.

[10] F. Digne, J. Michel, Representations of Finite Groups of Lie Type, London Mathematical Society Student Texts, vol. 21, Cambridge University Press, 1991.

[11] H. Enomoto, The characters of the finite symplectic group $\operatorname{Sp}(4, q), q=2^{f}$, Osaka J. Math. 9 (1972), pp. 75-94.

[12] W. Feit, Extending Steinberg characters, Contemp. Math., 153 (1993), pp. 19.

[13] W. Feit, On large Zsigmondy primes, Proc. Amer. Math. Soc.102, 1988, pp. 29-36.

[14] W. Feit, G. Seitz, On finite rational groups and related topics, Illinois J. Math. Volume 33, Issue 1 (1989), pp. 103-131.

[15] D. Frohardt, K. Magaard, Composition factors of monodromy groups, Annals of Mathematics, 154 (2001), pp. 327-345. 
[16] P. C. Gager, Maximal tori in finite groups of lie type, PhD thesis (1973), U. of Warwick.

[17] The GAP group, GAP - groups, algorithms, and programming, Version 4.4, 2004, http://www.gap-system.org.

[18] Y. Ge, Elementary properties of cyclotomic polynomials, Mathematical Reflections 2, 2008.

[19] M. Geck, G. Hiss, F. Lübeck, G. Malle, G. Pfeiffer, CHEVIE - A system for computing and processing generic character tables for finite groups of Lie type, Weyl groups and Hecke algebras. Appl. Algebra Engrg. Comm. Comput., 7 (1996), pp. 175-210.

[20] D. Gorenstein, Finite Groups, AMS Chelsea Publishing, 1968.

[21] D. Gorenstein, R. Lyons, R. Solomon, The Classification of the Finite Simple Groups, Number 3, Mathematical Surveys and Monographs, Amer. Math. Soc., Providence, 1994.

[22] R. Gow, Groups whose characters are rational-valued, J. Algebra 40, 1976, pp. 280-299.

[23] G. Hiss, Regular and semisimple blocks of finite reductive groups, J. London Math. Soc., 1990, pp. 63-68.

[24] G. Hiss, G. Malle, Low-dimensional representations of special unitary groups, J. Algebra 236, 2001, pp. 745-767.

[25] I. Martin Isaacs, Character Theory of Finite Groups, AMS Chelsea, Providence, 2006.

[26] G. James, The Irreducible Representations of the Finite General Linear Groups, 1986.

[27] W. M. Kantor, Prime power graphs for groups of Lie type, J. Algebra 247, 2002, pp. 370-434.

[28] P. Kleidman, Maximal subgroups of the finite 8-dimensional orthogonal groups $P \Omega_{8}^{+}(q)$ and their automorphism groups, J. Algebra 110, 1987, pp. 173-242.

[29] P. Kleidman, M. Liebeck, The Subgroup Structure of the Finite Classical Groups, Cambridge Univ. Press, 1990.

[30] A.S. Kleshchev, P. H. Tiep, Representations of finite special linear groups in non-defining characteristic, Adv. Math. 220 (2009) pp. 478-504. 
[31] M. Liebeck, E. O’Brien, A. Shalev, P.H. Tiep, The Ore conjecture, J. Europ. Math. Soc. 12 (2010), pp. 939 -1008.

[32] K. Lux, H. Pahlings, Representations of Groups: A Computational Approach, Cambridge Univ. Press, 2010.

[33] G. Malle, B. H. Matzat Inverse Galois Theory, Springer-Verlag Berlin Heidelberg, 1999.

[34] G. Malle, D. Testerman, Linear Algebraic Groups and Finite Groups of Lie Type, Cambridge Univ. Press, 2011.

[35] S. Marinelli, P. H. Tiep, Zeros of real irreducible characters of finite groups, Algebra and Number Theory, Volume 7 No. 3 (2013), pp. 567-593.

[36] A. Moreto, P. H. Tiep, Prime divisors of character degrees, J. Group Theory 11 (2008), pp. 341 - 356.

[37] G. Navarro, Characters and Blocks of Finite Groups, Cambridge Univ. Press, 1998.

[38] G. Navarro, P. H. Tiep, Characters of relative p'-degree over normal subgroups, Annals of Mathematics, 178 (2013), pp. 1135 - 1171.

[39] G. Navarro, P. H. Tiep, Degrees of rational characters of finite groups, Adv. Math. 224 (2010), pp. 1121 - 1142.

[40] G. Navarro, P. H. Tiep, Rational irreducible characters and rational conjugacy classes in finite groups, Trans. Amer. Math.Soc. 360 (2008) pp. 2443-2465.

[41] M. Roitman, On Zsigmondy primes, Proc. Amer. Math. Soc., Vol. 125, Number 7, 1997, pp. 1913-1919.

[42] T. A. Springer, R. Steinberg, Conjugacy Classes, in Seminar on algebraic groups and related finite groups (A. Borel et al., eds.), pp. 167-266. Lecture Notes in Mathematics 131, Springer, Berlin, 1970.

[43] B. Srinivasan, The characters of the finite symplectic group $\operatorname{Sp}(4, q)$, Trans. Amer. Math. Soc. 131, No. 2 (1968), pp. 488-525.

[44] R. Steinberg, Collected Papers, Amer. Math. Soc., Vol. 7, 1997.

[45] J. Tent, Quadratic rational solvable groups, J. Algebra 363, 2012, pp. 73-82.

[46] J. G. Thompson, Composition factors of rational finite groups, J. Algebra 319, 2008, pp. 558-594. 
[47] P. H. Tiep, A.E. Zalesskii, Minimal characters of the finite classical groups, Comm. Algebra 24 (1996) pp. 2093-2167.

[48] P. H. Tiep, A.E. Zalesskii, Some characterizations of the Weil representations of the symplectic and unitary groups, J. Algebra 192 (1997) pp. 130-165.

[49] T. S. Weigel, Generation of exceptional groups of Lie-type, Geometriae Dedicata, Volume 41, Issue 1, pp. 63-87, January 1992.

[50] A. V. Zavarnitsine, The structure of maximal tori in spin groups, Sib. Math. J. 56, N3 (2015) pp. 425-434.

[51] K. Zsigmondy, Zur Theorie der Potenzreste, Monatsch. Math. Phys. 3, 1892, pp. 265-284. 